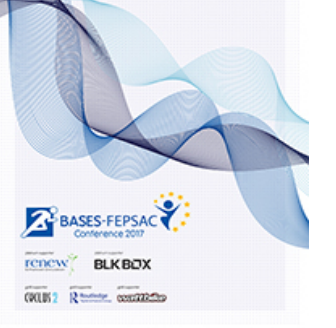

ISSN: 0264-0414 (Print) 1466-447X (Online) Journal homepage: http://www.tandfonline.com/loi/rjsp20

\title{
BASES Conference 2017 - Programme and Abstracts
}

To cite this article: (2017) BASES Conference 2017 - Programme and Abstracts, Journal of Sports Sciences, 35:sup1, 1-119, DOI: 10.1080/02640414.2017.1378421

To link to this article: https://doi.org/10.1080/02640414.2017.1378421

曲 Published online: 14 Nov 2017.

Submit your article to this journal 중

LII Article views: 1314

Q View related articles $\sqsubset$

View Crossmark data ¿ 


\section{Programme and Abstracts}

25 BASES-FEPSAC

renew"; BLKBLX

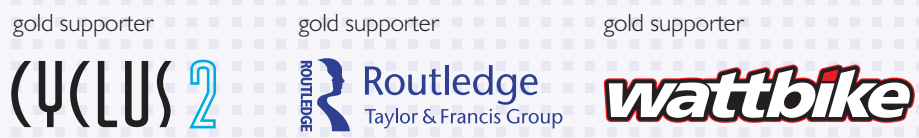




\section{BASES 2018}
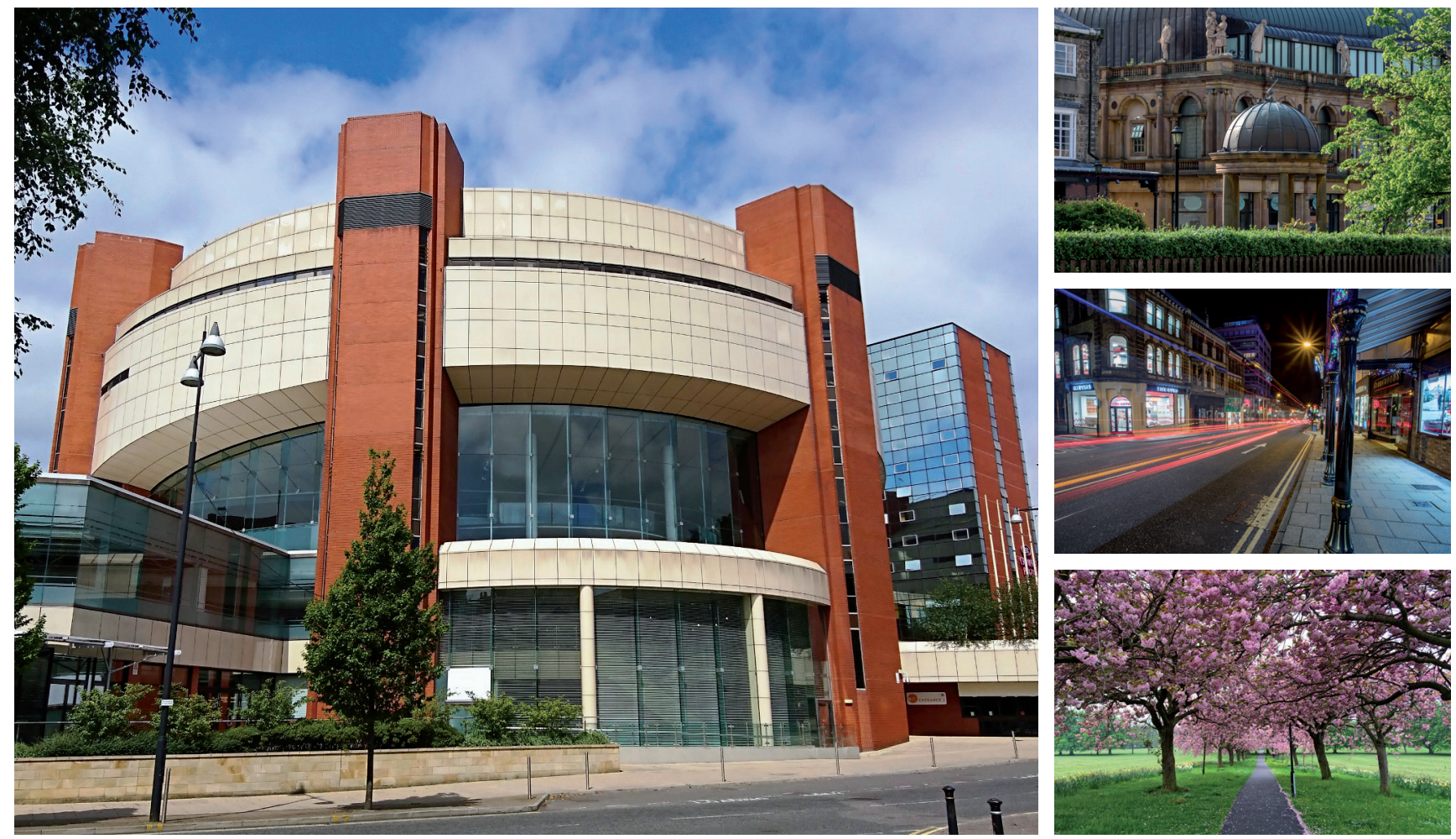

\section{Harrogate Convention Centre, Yorkshire, United Kingdom}

\section{Visit the fascinating Victorian spa town \\ of Harrogate in the heart of Yorkshire}

\section{Tuesday 27 - Wednesday 28 November 2018}

\section{Abstract submission deadlines}

Free Communications: Friday Ist June 2018

Posters and 5 slides in 5 minutes: Monday 2nd July 2018

\section{Early bird delegate rates}

Available until Friday 14th September 2018

For further information

\section{www.basesconference.co.uk}

y @basesuk f /BASESUK 0 bases_uk in BASESUK
- Two-day sport and exercise science programme packed with world-leading insight and experience

- Present your research (free communication and poster sessions) and gain a publication in the Journal of Sports Sciences

- Rub shoulders with world-leading experts

- Open doors to opportunities via the largest UK sport and exercise science network

- Engaging content including invited keynotes and symposia

- Five BASES re-accreditation credits per day

- Awards available to reward outstanding research 


\section{Contents}

Welcome

Dr Keith Tolfrey FBASES - Chair of the British Association of Sport and Exercise Sciences (BASES)

and Dr Anne-Marie Elbe - President of the European Federation of Sport Psychology (FEPSAC)

Programme overview

Prof Greg Whyte OBE, FBASES - Chair of the Scientific Programme Committee

Conference programme......

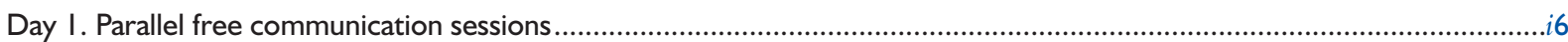

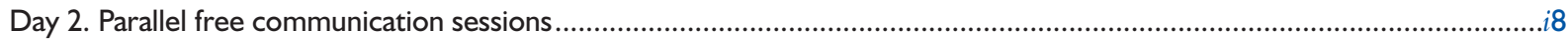

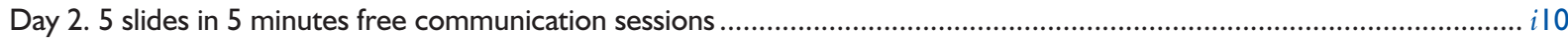

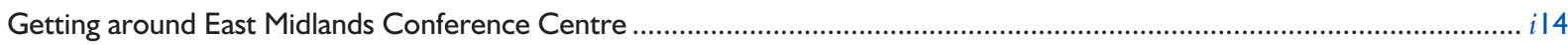

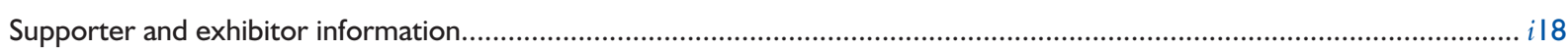

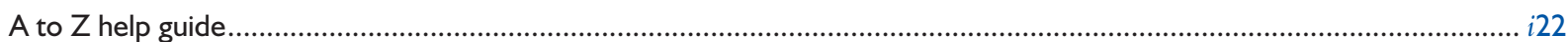

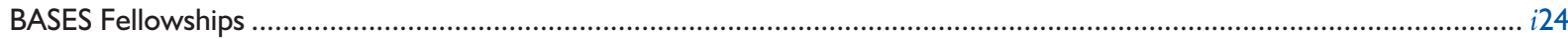

Day I

Invited keynote: Developing a high performance culture: recommendations for research and practice......

Dr Angus Mugford

Invited symposium: Physical activity and sedentary behaviour: measurement, interpretation and implementation ..... Prof Adrian Bauman and Prof Dylan Thompson

Invited symposium: The BASES expert statement highlight session on performance at the extremes: high, hot and cold Prof Greg Whyte OBE, FBASES, Dr jo Corbett, Prof Mike Tipton and Mark Homer

Invited symposium: Mental health problems in elite athletes: systems, challenges and recommendations for practice....

Prof Jens Kleinert, Dr Göran Kenttä, Cédric Quignon-Fleuret and Dr Kate Goodger

Invited symposium: The adaptive response of human skeletal muscle to physical (in)activity: considerations for nutritional optimisation..... Dr Benjamin Wall and Dr Oliver Witard

Invited symposium: Biomechanical and neuromuscular challenges during ageing and disuse Dr Ramona Ritzmann and Dr Claudine Lamoth

Invited symposium: Challenges in the provision of psychology support for performance excellence during the Rio Olympic and Paralympic Games.... Prof Paul Wylleman and Dr Kristoffer Henriksen

Invited symposium: Interdisciplinary research on physical activity interventions targeting obesity and smoking cessation ...... $i 33$ Prof Antonis Hatzigeorgiadis and Dr Anne-Marie Elbe

Day 2

Invited keynote: Virtual reality technology: what use can it have in sport?..... Prof Cathy Craig

Invited symposium: Exercise in older adults Prof Steve Harridge and Dr Katherine Brooke-Wavell

Invited symposium: The BASES expert statement session on inadvertent doping in sport: protecting the athlete, the practitioner and the profession... Prof Susan Backhouse FBASES, Dr lan Boardley and Mike Stow

Invited symposium: Sport psychophysiology.... Dr Maurizio Bertollo, Prof Lorenza Colzato and Prof Christopher Ring

Nutrition, exercise and appetite: hungry for more? Prof Emma Stevenson and Dr Lewis James

Invited symposium: Analysing, modelling and improving Olympic performance Prof Gareth Irwin and Prof Bert Otten

Invited symposium: Uncovering the process of a strength-based approach to mental skills training with young people Dr Jennifer Cumming and Dr Camilla Knight

Invited symposium: Direct perception in applied contexts..... Prof Cathy Craig, Prof Rita Cordovil, Prof Keith Davids and Dr Rita de Olivera

Invited keynote: Exercise versus pharma: what is the future of healthcare? Prof Sir Muir Gray CBE

Abstracts

Routledge

The Programme and Abstracts booklet is published in association with Journal of Sports Sciences, published by Taylor \& Francis Grou Routledge, and will be available as a supplement issue on the journal website at: www.tandfonline.com/rjsp 


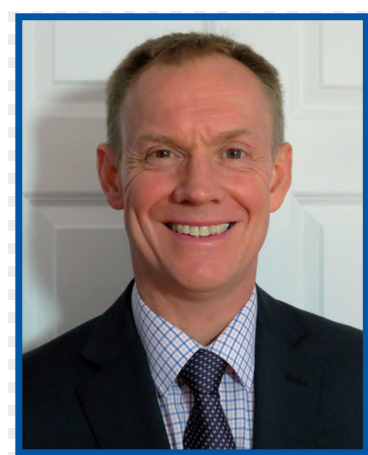

Dr Keith Tolfrey FBASES

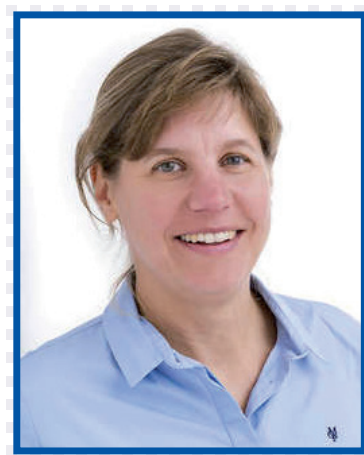

Dr Anne-Marie Elbe

Scientific Programme

Committee

- Prof Greg Whyte OBE, FBASES (Chair),

Liverpool John Moore's University

- Prof Keith Stokes FBASES (Deputy Chair),

University of Bath

- Prof Chris Harwood FBASES Loughborough University

- Dr Florentina Hettinga, University of Essex

- Prof David Stensel, Loughborough University

- Adam Hawkey, Southampton Solent University

- Prof Emma Stevenson, Newcastle University

- Prof Markus Raab, German Sport University, Cologne from Dr Keith Tolfrey FBASES

Chair of the British Association of Sport and Exercise Sciences (BASES)

and Dr Anne-Marie Elbe

President of the European Federation of Sport Psychology (FEPSAC)

On behalf of the British Association of Sport and Exercise Sciences and the European Federation of Sport Psychology, we welcome you to the BASES-FEPSAC Conference 2017. This will be the first joint conference between BASES and FEPSAC and we are both looking forward to what promises to be an exciting programme packed full of world-leading content. From keynote speakers, symposia, posters and free communications, this year's conference will deliver a wide variety of stimulating, thought-provoking and interactive sessions that will undoubtedly provide a memorable delegate experience. We are delighted to welcome Renew Health Limited and BLK BOX Fitness as our official platinum supporters; and Cyclus2, Routledge and Wattbike as official gold supporters.

Each day is brimming with content and insight, covering a wide variety of topics and presentation formats. The BASES Scientific Programme Committee, chaired by Professor Greg Whyte OBE, FBASES, has organised a series of captivating keynote speakers who will share insights on applied sport and exercise science in elite environments, discuss the implications and application of technological innovation in sport and explore the role of exercise in the future of healthcare. In partnering with FEPSAC, this year's programme is further enhanced by a psychology-specific strand focusing on interdisciplinary perspectives to sport and exercise science.

We extend a special welcome to each of our invited keynote speakers: Professor Cathy Craig from Queen's University, Belfast; Dr Angus Mugford from the Toronto Blue Jays, Canada and Professor Sir Muir Gray CBE from the University of Oxford.

Opportunities for socialising and networking play a key part in this event; we hope you will renew acquaintances and make new friends. To facilitate this, we have deliberately scheduled plenty of breaks and extended lunch periods. Day one will finish with the gala drinks reception and conference dinner, which promises to be a memorable evening of great food, entertainment and dancing (a FEPSAC tradition!).

The Banqueting and Exhibition Suite and Suite 2 will house the exhibitors, refreshments and posters; these spaces will be vibrant networking hubs to spend your time outside of the scheduled conference sessions. In addition to the five prestigious BASES awards presented last year, we are introducing two new awards at this year's conference, which seek to reward outstanding contributions to sport and exercise sciences by BASES members. Three awards recognising research excellence in sport and exercise psychology will be contested by FEPSAC student members. We are very grateful to the award sponsors: Cranlea, Human Kinetics, Routledge, Sportesse and Elsevier.

Thank you all for being part of this special event. We hope you take the time to share your extensive knowledge and wisdom with other delegates and that you have an inspiring and enjoyable conference experience. Enhanced Circulation platinum supporte BLKBLXX (U(LU) \& gold supporter

Routledge Taylor \& Francis Group gold supporter

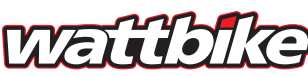




\title{
Programme overview
}

\author{
from Prof Greg Whyte OBE, FBASES
}

Chair of the Scientific Programme Committee

On behalf of the Scientific Programme Committee I am delighted to welcome you to the BASES-FEPSAC Conference 2017. Returning to the East Midlands Conference Centre, we have continued our commitment to provide a two-day programme of the highest quality science and opportunities to engage with distinguished national and international presenters and fellow delegates. The programme contains topics that reflect the broad range of interests of sport and exercise scientists presented by leading scientists and practitioners from around the globe.

For the first time, BASES have teamed up with FEPSAC to provide a unique opportunity for delegates from two leading organisations to participate in a world leading conference. We hope that the increased density of psychology-focused sessions promises to deliver a truly outstanding breadth of national and international specialists that will be a bonus for all delegates, whatever your scientific persuasion.

In keeping with recent conferences, the scientific committee have secured outstanding opening and closing sessions that you will not want to miss. These sessions will be led by outstanding individuals with vast experience of their respective fields. Opening the conference, Dr Angus Mugford, Head of Performance Services at the Toronto Blue Jays, flies in from North America to share his insights and experiences of creating a high-performance environment in professional sport. Professor Sir Muir Grey CBE, our closing session speaker, will address the contemporary issue of 'Exercise vs. Pharma: What is the future of healthcare?'. Sir Muir Gray CBE was the government's first Chief Knowledge Officer for the NHS and is a Professor and consultant of Public Health at Oxford University. Chaired by Professor Greg Whyte OBE, FBASES, the session will provide an opportunity for the audience to engage in a debate centred on one of the most contentious contemporary issues in modern healthcare provision.

The BASES Annual General Meeting (AGM) and the FEPSAC AGM will be held at the end of the first day of the conference. A full attendance at any AGM is always a challenge after a packed day of presentations, but please remember that attending your respective AGM provides a unique opportunity to comment on a range of issues that not only have an impact on your Association, but also on the standing of sport and exercise sciences within the UK and beyond. Therefore, it is in all our interests to participate in discussions that will shape the future direction and goals of both Associations.

The very essence of a scientific conference is sharing research findings. This is most effectively achieved through free communication presentations (including the new, dynamic 5 slides in 5 minutes sessions) and poster presentations. These sessions should be a twoway process. Authors present contemporary research findings, followed by exchanges that provide opportunities to discuss and debate results and interpretations, leading to enhanced understanding and new potential avenues of research. We encourage you all to support your fellow researchers by attending and participating in these sessions.

Finally, we hope that you have a productive and enjoyable time during the next two days, leaving even more enthusiastic about your commitment to sport and exercise sciences.

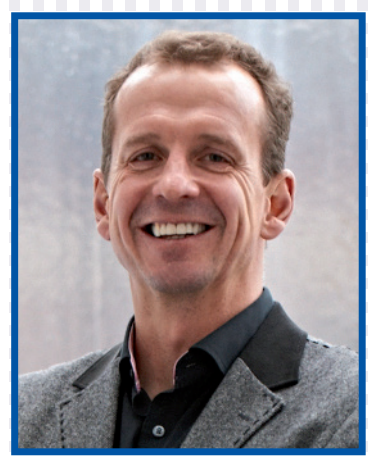

Prof Greg White OBE, FBASES

Abstract Reviewers

- Dr Ibrahim Akubat

- Dr Rachel Arnold

- Prof Sue Backhouse FBASES

- Dr Maurizio Bertollo

- Dr David Broom FBASES

- Dr Jenny Burbage

- Dr Neil Clarke

- Dr Stewart Cotterill FBASES

- Dr Rita de Oliveira

- Dr Nadine Debois

- Prof Mike Duncan FBASES

- Dr Anne-Marie Elbe

- Adam Grainger

- Prof Chris Harwood FBASES

- Prof Antonis Hatzigeorgiadis

- Adam Hawkey

- Dr Shane Heffernan

- Dr Camilla Knight

- Prof Zoe Knowles FBASES

- Dr lan Lahart

- Dr Anthony Papathomas

- Dr John Perry

- Dr Mike Price FBASES

- Prof Markus Raab

- Dr Dan Robbins

- Dr Xavier Sanchez

- Dr Richard Thelwell FBASES

- Dr Sandy Willmott

- Julie York

exhibitors

\begin{tabular}{|c|c|c|c|c|c|}
\hline 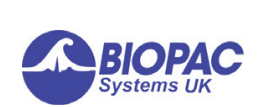 & BOB & COSMED & CRANLEA (O) & Oᄋ FIRSTBEAT & : \\
\hline TSuman kinetics & $\underset{\text { measure. analyea innovate. }}{\mathrm{KISTLER}}$ & Lake Image Systems & $2 \sqrt{8} \begin{array}{c}\text { medical graphics } \\
\text { unumber }\end{array}$ & P3 & \\
\hline sportingedge & TANDTA & & ucichan & Vyaire & 9. Wo \\
\hline
\end{tabular}




\begin{tabular}{|c|c|}
\hline 07:30-08:45 & Exhibitor set up \\
\hline 09:00 & Registration and drinks \\
\hline 10:00 & $\begin{array}{l}\text { Opening address } \\
\text { Welcome from Dr Keith Tolfrey FBASES, Cha }\end{array}$ \\
\hline $10: 10-11: 10$ & $\begin{array}{l}\text { Invited keynote: Developing a high perfor } \\
\text { Dr Angus Mugford, Head of Performance Ser } \\
\text { This keynote will draw upon insights and experie } \\
\text { focusing on the challenges and opportunities tha } \\
\text { interdisciplinary work in the context of professior }\end{array}$ \\
\hline II:I0-II:30 & Poster viewing, exhibition and refreshn \\
\hline \multirow[t]{5}{*}{ II:30-12:45 } & Parallel invited symposia \\
\hline & $\begin{array}{l}\text { Physical activity and sedentary } \\
\text { behaviour: measurement, } \\
\text { interpretation and implementation }\end{array}$ \\
\hline & $\begin{array}{l}\text { Prof Adrian Bauman, The University } \\
\text { of Sydney, Australia and Prof Dylan } \\
\text { Thompson, University of Bath }\end{array}$ \\
\hline & Chair: Prof Mike Duncan FBASES \\
\hline & $\begin{array}{l}\text { In this session Prof Dylan Thompson will explain } \\
\text { the multidimensional nature of physical activity and } \\
\text { discuss some new techniques and approaches for } \\
\text { physical activity monitoring. Prof Adrian Bauman } \\
\text { will critically evaluate the evidence relating to sitting } \\
\text { behaviour and health outcomes and will assess } \\
\text { the challenges inherent in translating research } \\
\text { into practice. }\end{array}$ \\
\hline
\end{tabular}

DI.S2.I. Suite I
Performance at the extremes: high, hot and cold

BASES expert statement highlight session

Prof Greg Whyte OBE, FBASES, Dr Jo Corbett, University of Portsmouth, Prof Mike Tipton, University of Portsmouth and Mark Homer, British Rowing Chair: Prof Greg Whyte OBE, FBASES

The ability to cope with the challenges of extreme environments is crucial for performance and health Our understanding of the complex interaction of the human body in extreme environments has continued to evolve, leading to athletes pushing the limits of human performance. This session is a roundtable discussion of the BASES expert statement on performance and health in high, hot and cold environments.

Conference Theatre

\section{$12: 45$}

13:15-14:15

14:15-15:45

15:45-16:05

16:05-17:20

$17: 30-18: 30$
Lunch

\section{Poster discussions and exhibition}

Parallel free communication sessions

Exhibition and refreshments

\section{Parallel invited symposia}

The adaptive response of human skeletal muscle to physical (in)activity: considerations for nutritional optimisation Dr Benjamin Wall, University of Exeter and Dr Oliver Witard, University of Stirling Chair: Prof Emma Stevenson Skeletal muscle tissue exhibits great plasticity depending on the mechanical strain imposed upon it. This session will discuss the physiological mechanisms underlying the adaptive response to increased, and decreased, physical activity; and address how the adaptive response to structured exercise training can be optimised via considerations relating to dietary protein. The session's conclusions will aim to provide upto-date recommendations for dietary protein intake in endurance and resistance trained athletes.

\section{DI.S4.I. Suite I}

BASES Annual General Meeting
Biomechanical and neuromuscular challenge during ageing and disuse Dr Ramona Ritzmann, University of Freiburg, Germany and Dr Claudine Lamoth, University of Groningen, Netherlands Chair: Dr Florentina Hettinga This session will provide an insight into adaptations in response to ageing, with a particular focus on biomechanical and neuromuscular changes. Data obtained about the mechanisms underlying degradations in response to inactivity during bed rest and immobilisation will be systematically reviewed and various countermeasures designed to reduce these effects will be discussed.

\section{DI.S2.2. Suite 3}

Mental health problems in elite athletes: systems, challenges and recommendations for practice Prof Jens Kleinert, German Sport University, Cologne, Dr Göran Kenttä, Swedish School of Sport and Health Sciences, Cédric Quignon-Fleuret, Institute of Sport, France and Dr Kate Goodger, Chimp Management Chair: Dr Karin Moesch

This roundtable focuses on mental health problems in elite athletes. Participants from different countries will provide insight into how elite athletes with mental health problems can be supported, with a focus on prevention, detection and treatment of mental health problems in this specific population. Recommendations will be discussed to further enhance clinically oriented sport psychology services.

Banqueting and Exhibition Suite/Suite 2

Banqueting and Exhibition Suite/Suite 2

Challenges in the provision of psychology support for performance excellence during the Rio Olympic and Paralympic Games

Prof Paul Wylleman, Vrije Universiteit Brussel, Belgium and Dr Kristoffer Henriksen, University of Southern Denmark

Chair: Dr Rachel Arnold

This session will highlight the contrasting challenges regarding the content, organisation and quality of support provided to Olympic and Paralympic athletes by team psychologists at Rio 20 16. Drawing from their experiences, critical insights will be offered on support provision; the organisation and effects of a solid performance culture; and on the competency profiles of practitioners working with elite athletes.
Interdisciplinary research on physical activity interventions targeting obesity and smoking cessation

Prof Antonis Hatzigeorgiadis, University of Thessaly, Greece and Dr Anne-Marie Elbe, University of Copenhagen, Denmark

Chair: Dr Anne-Marie Elbe

This session will cover interdisciplinary research conducted on two physical activity interventions. The first targets smoking cessation and the collection of psychological and biochemical data. The second investigates the effects of an intense lifestyle intervention for individuals with obesity, combining a physiological, psychological and sociological research perspective. Advantages and challenges of interdisciplinary physical activity research will be outlined.

DI.S4.2. Suite 4 DI.S4.3. Conference Theatre
DI.S4.4. Suite 3

FEPSAC Annual General Meeting

Gala drinks reception

Atrium

Conference dinner 
09:15-10:10

Invited keynote: Virtual reality technology: what use can it have in sport?

Prof Cathy Craig, Queen's University Belfast, N. Ireland Chair: Prof Markus Raab

This keynote will show how immersive, interactive virtual reality technology is used to study decision-making in sport. The versatility of this technology means it can be easily applied to many sports and allows for the accurate recording of players' movement responses. By manipulating the visual information presented to players in the virtual world and subsequently measuring when and how they respond, new insights into players' decision-making abilities can be obtained.

D2.S2. Conference Theatre

$10.15-11: 10$

\section{Parallel invited symposia}

Exercise in older adults

Prof Steve Harridge, Kings College London and Dr Katherine Brooke-Wavell, Loughborough University

Chair: Dr Miranda Armstrong

In this session Prof Steve Harridge will examine the decline in physiological systems that occur with ageing and discuss studies in master's athletes to assess the extent to which declines in functional capacity are due to ageing per se versus physical inactivity. Dr Katherine Brooke-Wavell will then share her knowledge on the potential for exercise to increase bone strength and reduce the risk of falls and osteoporotic fractures in older adults. This will include an examination of the different types and intensities of exercise for increasing bone density.

\section{Inadvertent doping in sport:}

protecting the athlete, the practitioner and the profession BASES expert statement highlight session

Prof Susan Backhouse FBASES, Leeds Beckett University, Dr lan Boardley, University of Birmingham and Mike Stow, English Institute of Sport

Chair: Prof Susan Backhouse FBASES

This session will consider the controversial issue of doping in sport, and in particular, the unintentional use of prohibited substances or methods. This session will use collaborative technology to activate the BASES community and gauge their stance on the BASES expert statement on inadvertent doping in sport.

\section{Sport psychophysiology \\ Dr Maurizio Bertollo, University of Chieti-Pescara, Italy, Prof Lorenza Colzato Leiden University, Netherlands and Prof Christopher Ring, University of Birmingham \\ Chair: Dr Maurizio Bertollo \\ This session will provide an overview of the psychophysiological features of optimal and suboptimal performance in sport. Presenters will discuss the neural markers underlying performance experience and the effect of non-invasive brain stimulation on performance. The conclusion of this synthesis is applicable within performance enhancement and mental training programmes, providing a comprehensive perspective on psychophysiological intervention in sport.}

D2.S3. I. Suite I

D2.S3.2. Suite 3

D2.S3.3. Conference Theatre

Poster viewing, exhibition and refreshments

Banqueting and Exhibition Suite/Suite 2

Parallel free communication sessions

II:30-12:45

$12: 45$

12:50-13:45

|3:45-14:45

|4:55-|15:45
Lunch

Poster discussions and exhibition

Banqueting and Exhibition Suite/Suite 2

\section{Parallel invited symposia}

Nutrition, exercise and appetite: hungry for more?

Prof Emma Stevenson,

Newcastle University and Dr Lewis James, Loughborough University Chair: Dr Mike Price FBASES This session will focus on the impact of alterations in energy balance through energy restriction and exercise on appetite and energy balance regulation. There will be particular focus on novel dietary strategies to attenuate energy balance, including intermittent severe energy restriction and time restricted feeding.

Analysing, modelling
and improving Olympic
performance
Prof Gareth Irwin, Cardiff
Metropolitan University and
Prof Bert Otten, University of
Groningen, Netherlands
Chair: Adam Hawkey
Many aspects of Olympic performance
can be analysed with motion capture
and simulated with custom models.
Drawing on current research
examples from amputee sprinting,
gymnastics, track cycling and speed
skating, this session will demonstrate
how ecologically valid research in
biomechanics, aerodynamics and
motor control can enhance the
performance of Olympic athletes.

Analysing, modelling oving Olympic Prof Gareth Irwin, Cardiff Metropolitan University and Prof Bert Otten, University of Groningen, Netherlands an be anaysed wh motion capture and simulated with custom models. Drawing on current research mples from amputee sprinting, performance of Olympic athletes.

\section{Uncovering the process of a strength-based approach to mental skills training with young people \\ Dr Jennifer Cumming, University of Birmingham and Dr Camilla Knight, Swansea University \\ Chair: Prof Zoe Knowles FBASES \\ This session will explore the delivery and evaluation of strength-based approaches to mental skills training in elite sport and hard to reach populations. Strategies and activities will be shared which have facilitated the application of mental skills training across various settings, as well as insights into the successes and challenges encountered in delivering strength-based mental skills training programmes.}

Direct perception in applied contexts Prof Cathy Craig, Queen's University Belfast, N. Ireland, Prof Rita Cordovil, University of Lisbon, Portugal, Dr Rita de Oliveira, London South Bank University and Prof Keith Davids, Sheffield Hallam University

Chair: Dr Rita De Oliveira

The direct perception approach posits that perception is unmediate by cognition, with consequences to how we organise both research and practice. Talks in this symposium will explain how key concepts from this approach can be used to guide research methodology and practical applications in exercise and sport.

D2.S5.4. Suite

Invited keynote: Exercise versus pharma: what is the future of healthcare?

Prof Sir Muir Gray CBE, Nuffield Department of Surgical Sciences, University of Oxford Chair: Prof Greg Whyte OBE, FBASES Prof Greg Whyte OBE, FBASES will host a discussion with Prof Sir Muir Gray CBE on the role of exercise in health and primary care. Expect stimulating debate and audience participation on the role of exercise in the prevention, treatment and management of disease and views on how the health service can move forward.

D2.S6. Conference Theatre 


\section{Day I. Parallel free communication sessions}

Physical Activity for Health

Chair: Prof David Stensel

\begin{tabular}{|c|c|c|}
\hline 14:15 & DI.S3.I(I) & $\begin{array}{l}\text { Cardiometabolic responses to interrupting prolonged sitting with high-intensity } \\
\text { physical activity versus a continuous moderate-intensity physical activity bout } \\
\text { Daniel Bailey, Benjamin Maylor, Charlie Orton \& Julia Zakrzewski-Fruer }\end{array}$ \\
\hline $14: 30$ & DI.S3.I(2) & $\begin{array}{l}\text { The utility of the Supine to Stand test as a measure of functional motor competence } \\
\text { in children aged 5-9 years old } \\
\text { Michael Duncan, Chelsey Lawson, Leanne Walker, David Stodden \& Emma Eyre }\end{array}$ \\
\hline 14:45 & DI.S3.I(3) & $\begin{array}{l}\text { The effect of a shortened sprint, reduced exertion high-intensity interval training (REHIT) } \\
\text { protocol on affective response and peak oxygen uptake } \\
\text { Matthew Haines }\end{array}$ \\
\hline 15:00 & DI.S3.I(4) & $\begin{array}{l}\text { Walking in short bouts throughout the day reduces postprandial triglycerides in } \\
\text { older women with elevated fasting triglycerides } \\
\text { Kyoko Kashiwabara, Tetsuhiro Kidokoro, Takuma Yanaoka, Stephen F. Burns, David J. Stensel \& Masashi Miyashita }\end{array}$ \\
\hline 15:15 & DI.S3.I(5) & $\begin{array}{l}\text { Comparative study of the effect of aquatic plyometric training versus land-based } \\
\text { exercises on bone mineral density in physically active women } \\
\text { Manuel A. Riveros, Jose I. Garcia, Adriana R. Gutierez, Carlos A. Collazos \& Oscar F. Rubiano }\end{array}$ \\
\hline 15:30 & DI.S3.I(6) & $\begin{array}{l}\text { Association between breakfast frequency and physical activity and sedentary time during } \\
\text { different times of the day in children from I } \mathbf{2} \text { countries } \\
\text { Julia K. Zakrzewski-Fruer, Fiona B. Gillison, Sean Cumming, Peter T. Katzmarzyk, Stephanie T. Broyles, } \\
\text { Catherine M. Champagne, Jean-Philippe Chaput, Kara D. Denstel, Mikael Fogelholm, Gang Hu, Kuriyan Rebecca, } \\
\text { Anura V. Kurpad, Estelle V. Lambert, Carol Maher, José Maia, Victor Matsudo, Emily F. Mire, Tim Olds, } \\
\text { Vincent Onywera, Olga L. Sarmiento, Mark S. Tremblay, Catrine Tudor-Locke, Pei Zhao \& Martyn Standage }\end{array}$ \\
\hline
\end{tabular}

Physiology and Nutrition

Chair: Dr Caroline Sunderland FBASES

\begin{tabular}{|l|l|l|}
\hline I4:I5-I5:45, DI.S3.2. Gallery Suite \\
\hline I4:I5 & DI.S3.2(I) & $\begin{array}{l}\text { A comparison of neural adaptations after twelve weeks and } \\
\text { four years of resistance training } \\
\text { Thomas G. Balshaw, Garry J. Massey, Thomas M. Maden-Wilkinson, } \\
\text { Marcel B. Lanza \& Jonathan P. Folland }\end{array}$ \\
\hline 14:30 & DI.S3.2(2) & $\begin{array}{l}\text { Exercise-induced muscle damage and time-course of recovery in young } \\
\text { and middle-aged males } \\
\text { John F. T. Fernandes, Kevin L. Lamb \& Craig Twist }\end{array}$ \\
\hline 14:45 & DI.S3.2(3) & $\begin{array}{l}\text { Elevated baseline work-rate slows pulmonary oxygen uptake kinetics, decreases } \\
\text { critical power and increases W' during supine cycle exercise } \\
\text { Richie Goulding, Denise Roche \& Simon Marwood }\end{array}$ \\
\hline 15:00 & DI.S3.2(4) & $\begin{array}{l}\text { Catecholamines, but not the sit-up tilt test, are predictive of the inflammatory } \\
\text { response to a wheelchair half-marathon } \\
\text { Sven Hoekstra, Yoshi Kamijo, Tokio Kinoshita, Ben Stephenson, Nicolette Bishop, } \\
\text { Christof Leicht, Vicky Tolfrey \& Fumihiro Tajima }\end{array}$ \\
\hline 15:I5 & DI.S3.2(5) & $\begin{array}{l}\text { Acute effects of low-load systemic and localised hypoxic resistance training on skeletal muscle } \\
\text { morphology, metabolic stress and muscle tissue oxygenation } \\
\text { Ryan Marshall, William D. Sheldon \& Elizabeth M. Board }\end{array}$ \\
\hline 15:30 & DI.S3.2(6) & $\begin{array}{l}\text { The effects of crank rate on the physiological and perceptual responses } \\
\text { to high intensity intermittent upper body exercise } \\
\text { Mike Price, Paul Smith \& Gabriel Gennuso }\end{array}$ \\
\hline
\end{tabular}

Psychology (Session I)

Chair: Prof Chris Harwood FBASES

\section{4:I5-I5:45, DI.S3.3. Conference Theatre}

\begin{tabular}{|l|l|l|}
\hline I4:I5 & DI.S3.3(I) & $\begin{array}{l}\text { The motivation of elite athletes: a mental health perspective } \\
\text { Rachel B. Sheehan, Matthew P. Herring \& Mark J. Campbell }\end{array}$ \\
\hline I4:30 & DI.S3.3(2) & $\begin{array}{l}\text { “You have to be mental to jump off a board any way": elite divers' conceptualizations } \\
\text { and perceptions of mental health } \\
\text { Melissa Coyle, Paul Gorczynski \& Kass Gibson }\end{array}$ \\
\hline $\mathbf{1 4 : 4 5}$ & DI.S3.3(3) & $\begin{array}{l}\text { Wellbeing of practitioners travelling with athletes: balancing between sanity and insanity } \\
\text { Kotryna Fraser \& Moji Shahvail }\end{array}$ \\
\hline
\end{tabular}




\section{|4:15-I5:45, DI.S3.3. Conference Theatre}

\begin{tabular}{|l|l|l|}
\hline I5:00 & DI.S3.3(4) & $\begin{array}{l}\text { A brief sports-based mental health literacy programme to increase help-seeking } \\
\text { among adolescent male athletes: a cluster-randomised controlled trial } \\
\text { Sarah Liddle, Frank Deane \& Stewart Vella }\end{array}$ \\
\hline I5:I5 & DI.S3.3(5) & $\begin{array}{l}\text { Development and qualitative evaluation of a website and mobile-app supported } \\
\text { intervention to increase resilience and wellbeing in youth sport } \\
\text { Christian Swann, Michael Noetel, Andrea Fogarty, Richard Keegan, Katherine Tamminen, } \\
\text { Mustafa Sarkar, Helen Ferguson \& Matthew Schweickle }\end{array}$ \\
\hline I5:30 & DI.S3.3(6) & $\begin{array}{l}\text { I am great and I want to dominate: narcissism and performance under stress } \\
\text { Shuge Zhang, Ross Roberts, Andrew Cooke \& Tim Woodman }\end{array}$ \\
\hline
\end{tabular}

\section{Psychology (Session 2)}

Chair: Prof Zoe Knowles FBASES

\section{|4:I5-I5:45, DI.S3.4. Suite 3}

\begin{tabular}{|c|c|c|}
\hline $14: 15$ & DI.S3.4(I) & $\begin{array}{l}\text { Attentional focus and metacognitive processes in recreational endurance runners: } \\
\text { exploring the impact of cognitive strategies on longer-term activity adherence } \\
\text { Noel Brick, Mark Campbell, Rachel Sheehan \& Tadhg Maclntyre }\end{array}$ \\
\hline $14: 30$ & DI.S3.4(2) & $\begin{array}{l}\text { The psychological characteristics of developing excellence: academy coaches' reflections } \\
\text { on elite youth athletes, practical implications and actions two years on } \\
\text { Matthew Cullen \& Jonathan Glynn }\end{array}$ \\
\hline $14: 45$ & DI.S3.4(3) & $\begin{array}{l}\text { Is every type of choice equal for youth rope skippers: the role of order and equally } \\
\text { (un)attractive options in promoting task perception, engagement and intended perseverance } \\
\text { Gert-Jan De Muynck, Bart Soenens, Lotte Degraeuwe \& Maarten Vansteenkiste }\end{array}$ \\
\hline 15:00 & DI.S3.4(4) & $\begin{array}{l}\text { The influence of music and music-video on self-selected exercise intensity } \\
\text { and psychological response to treadmill exercise } \\
\text { Jasmin Hutchinson \& Jessica Ballister }\end{array}$ \\
\hline 15:15 & DI.S3.4(5) & $\begin{array}{l}\text { Music hath charms to soothe the savage beast but what of the savage workout? } \\
\text { Psychological and psychophysiological effects of recuperative music } \\
\text { Costas Karageorghis, Andrew Bruce, Suzanne Pottratz, Rebecca Stevens, Marcelo Bigliassi \& Mark Hamer }\end{array}$ \\
\hline 15:30 & DI.S3.4(6) & $\begin{array}{l}\text { Community level rugby union players' concussion experiences } \\
\text { Paul Sellars, Stephen Mellalieu \& Sharief Hendricks }\end{array}$ \\
\hline
\end{tabular}

\section{|4:I5-15:45, DI.S3.5. Suite I}

\begin{tabular}{|l|l|l|}
\hline 14:15 & DI.S3.5(I) & $\begin{array}{l}\text { Which measures of training load explain the change in neuromuscular } \\
\text { strength post-game in soccer players? } \\
\text { lbrahim Akubat, Chris Wilcox, Lee Taylor, Steve Barrett, Daniel Peart, Chris Towlson, James Bray, } \\
\text { Manuel Lapuente Sagarra, Tony Myers \& Grant Abt }\end{array}$ \\
\hline 14:30 & DI.S3.5(2) & $\begin{array}{l}\text { Effects of brain endurance training on endurance exercise performance } \\
\text { Neil Dallaway, Sam Lucas \& Christopher Ring }\end{array}$ \\
\hline 14:45 & DI.S3.5(3) & $\begin{array}{l}\text { Pacing and performance in head-to-head competition: external cues as invitations } \\
\text { for actions } \\
\text { Marco Konings \& Florentina Hettinga }\end{array}$ \\
\hline 15:00 & DI.S3.5(4) & $\begin{array}{l}\text { Exploring the barriers and enablers to implementing organizational-level change } \\
\text { within Olympic sport programmes } \\
\text { Conor Molan, Seamus Kelly, Rachel Arnold \& James Matthews }\end{array}$ \\
\hline 15:15 & DI.S3.5(5) & $\begin{array}{l}\text { The anaerobic power reserve and its predictive ability of short duration } \\
\text { maximal power output in professional road cyclists } \\
\text { Dajo Sanders \& Mathieu Heijboer }\end{array}$ \\
\hline 15:30 & DI.S3.5(6) & $\begin{array}{l}\text { The effects of post-activation potentiation on Counter Movement Jump in developmental } \\
\text { swimming athletes } \\
\text { Kirsten Grant, Mark Murray \& Jed McKernie }\end{array}$ \\
\hline
\end{tabular}




\section{Day 2. Parallel free communication sessions}

Biomechanics and Motor Behaviour

I I:30-12:45, D2.S4. I. Suite 4
Chair: Adam Hawkey

\begin{tabular}{|c|c|c|}
\hline I I:30 & D2.S4.I(I) & $\begin{array}{l}\text { Footstrike kinematic effects of motor learning strategies to retrain running gait } \\
\text { Marianne Gittoes, Kelly Ashford, Charlotte Michelmore \& Isabel Moore }\end{array}$ \\
\hline I I:45 & D2.S4.I(2) & $\begin{array}{l}\text { Influence of range of motion on muscle activity whilst using the } \\
\text { MuJo }{ }^{T M} \text { External Shoulder Machine } \\
\text { Victoria Jones, Peter Mundy, Neil Clarke \& Samuel Oxford }\end{array}$ \\
\hline $12: 00$ & D2.S4.I(3) & $\begin{array}{l}\text { The effect of knee joint angle on quadriceps explosive strength, and underpinning } \\
\text { neural and contractile determinants } \\
\text { Marcel B. Lanza, Thomas Balshaw \& Jonathan Folland }\end{array}$ \\
\hline $12: 15$ & D2.S4.I(4) & $\begin{array}{l}\text { Neural and hypertrophic adaptations to maximal eccentric versus } \\
\text { concentric training matched for total work } \\
\text { Sumiaki Maeo, Xiyao Shan, Shun Otsuka, Hiroaki Kanehisa \& Yasuo Kawakami }\end{array}$ \\
\hline $12: 30$ & D2.S4.I(5) & $\begin{array}{l}\text { KInfluence of a visual reference point for the hurdle on step regulation } \\
\text { and hurdle kinematics in gymnastics handspring vault } \\
\text { Apostolos Theodorou \& Georgios Ntallas }\end{array}$ \\
\hline
\end{tabular}

Chair: Dr Keith Tolfrey FBASES

\section{| I:30-12:45, D2.S4.2. Gallery Suite}

\begin{tabular}{|l|l|l|}
\hline I I:30 & D2.S4.2(I) & $\begin{array}{l}\text { Effects of consuming whey and casein protein post resistance exercise on } \\
\text { food intake and fullness in healthy males } \\
\text { David Broom, Alex Kerrigan, Elly Bott, Louis Havenhand \& James Lewis }\end{array}$ \\
\hline I I:45 & D2.S4.2(2) & $\begin{array}{l}\text { Cycling using immersive virtual reality enhances acute exercise tolerance } \\
\text { Jamie Highton, Thomas Williams, Gerard Nowlan, Serban Pop \& Craig Twist }\end{array}$ \\
\hline I2:00 & D2.S4.2(3) & $\begin{array}{l}\text { Reliability of ratings of perceived exertion during combined arm-leg ergometry } \\
\text { Mathew Hill, Michael Puddiford, Christopher Talbot \& Mike Price }\end{array}$ \\
\hline I2:I5 & D2.S4.2(4) & $\begin{array}{l}\text { Physiological effects of motor learning strategies to retrain running gait: a pilot study } \\
\text { Isabel Moore, Kelly Ashford, Charlotte Bitchell \& Marianne Gittoes }\end{array}$ \\
\hline I2:30 & D2.S4.2(5) & $\begin{array}{l}\text { Inter-individual variability in the appetite, acylated ghrelin and total peptide YY } \\
\text { response to acute exercise in young men: a replicated cross-over study } \\
\text { Fernanda Reistenbach-Goltz, Alice Thackray, James King, James Dorling, Greg Atkinson \& David Stensel }\end{array}$ \\
\hline
\end{tabular}

\section{Psychology (Session I)}

\section{Chair: Dr. Xavier Sanchez}

\section{I:30-I2:45, D2.S2.3. Conference Theatre}

\begin{tabular}{|l|l|l|}
\hline I I:30 & D2.S4.3(I) & $\begin{array}{l}\text { Pupillometry during golf putting: a new window on the cognitive mechanisms } \\
\text { underlying Quiet Eye } \\
\text { Mark Campbell, Aidan Moran, Sean Surmon, Liz Bressan \& lan Kenny }\end{array}$ \\
\hline I I:45 & D2.S4.3(2) & $\begin{array}{l}\text { Coach, athlete, and relational influences on athletes' attitudes, } \\
\text { intentions and willingness to dope } \\
\text { Paul Freeman, Di Fu, Daniel Madigan \& Tim Rees }\end{array}$ \\
\hline I 2:00 & D2.S4.3(3) & $\begin{array}{l}\text { Attention, heart rate and skilled motor performance: effects of task difficulty } \\
\text { Jennifer Henderson \& Christopher Ring }\end{array}$ \\
\hline I2:I5 & D2.S4.3(4) & $\begin{array}{l}\text { Differences in goal-directed self-talk use and functions between individual and team sport } \\
\text { athletes and between genders } \\
\text { Alexander T. Latinjak, Yago Ramis \& Miquel Torregrossa }\end{array}$ \\
\hline I2:30 & D2.S4.3(5) & $\begin{array}{l}\text { From Olympic rings to the circus ring: gymnasts' transition experiences into circus } \\
\text { Fleur van Rens \& Edson Filho }\end{array}$ \\
\hline
\end{tabular}


I I:30-12:45, D2.S4.4. Suite 3

\begin{tabular}{|l|l|l|}
\hline I I:30 & D2.S4.4(I) & $\begin{array}{l}\text { An investigation into the sport ethic of equestrian eventing } \\
\text { Deborah Brewer \& Clare Rhoden }\end{array}$ \\
\hline I I:45 & D2.S4.4(2) & $\begin{array}{l}\text { “When do I stop her?" Exploring overuse injuries through } \\
\text { the eyes of young gymnasts' parents } \\
\text { Francesca Cavallerio, Ross Wadey \& Christopher R. D. Wagstaff }\end{array}$ \\
\hline $\mathbf{I 2 : 0 0}$ & $\mathbf{D 2 . S 4 . 4 ( 3 )}$ & $\begin{array}{l}\text { Conceptualizing sporting experiences as romantic attachments: } \\
\text { a mixed methods exploration of loving the game } \\
\text { Alex Hodge, Emily Oliver \& David Eccles }\end{array}$ \\
\hline $\mathbf{I 2 : 1 5}$ & D2.S4.4(4) & $\begin{array}{l}\text { Physical abuse in sport: classifying types \& understanding impact } \\
\text { Emma Kavanagh, Lorraine Brown \& lan Jones }\end{array}$ \\
\hline $\mathbf{I 2 : 3 0}$ & D2.S4.4(5) & $\begin{array}{l}\text { Experienced sport psychology consultant's engagement in supervision } \\
\text { as a tool to stay ethical } \\
\text { Lee-Ann Sharp, Ken Hodge \& Steve Danish }\end{array}$ \\
\hline
\end{tabular}

Sport and Performance

Chair: Dr Ibrahim Akubat

| I:30-12:45, D2.S4.5. Suite |

\begin{tabular}{|l|l|l|}
\hline I I:30 & D2.S4.5(I) & $\begin{array}{l}\text { Match demands in elite women's English Premiership rugby union } \\
\text { Eddie Bradley, Phil Sharpe, Bob Hogg \& David Archer }\end{array}$ \\
\hline I I:45 & D2.S4.5(2) & $\begin{array}{l}\text { An examination of a modified Yo-Yo test to measure intermittent } \\
\text { running performance in rugby players } \\
\text { Nick Dobbin, Samantha Moss, Jamie Highton \& Craig Twist }\end{array}$ \\
\hline I2:00 & D2.S4.5(3) & $\begin{array}{l}\text { Within and between-session reliability of jump performance } \\
\text { in elite rugby union players } \\
\text { Adam Grainger, Paul Comfort, John McMahon \& Paul Jones }\end{array}$ \\
\hline I2:I5 & D2.S4.5(4) & $\begin{array}{l}\text { Integration of internal and external training load for field based } \\
\text { measures of fitness in rugby union } \\
\text { Richard Taylor, Dajo Sanders \& lbrahim Akubat }\end{array}$ \\
\hline I2:30 & D2.S4.5(5) & $\begin{array}{l}\text { Exercise-induced muscle damage and changes in resting metabolic rate } \\
\text { after intermittent running with and without physical contact } \\
\text { Craig Twist, Joshua Lee \& Jamie Highton }\end{array}$ \\
\hline
\end{tabular}




\section{Day 2. 5 slides in 5 minutes free communication sessions}

\begin{tabular}{|c|c|c|}
\hline \multicolumn{2}{|c|}{$\begin{array}{l}\text { Biomechanics and Motor Behaviour / } \\
\text { Physiology and Nutrition }\end{array}$} & Chair: Dr Mike Price FBASES \\
\hline \multicolumn{3}{|c|}{ 08:I5-09:I0, D2.SI.I. Suite 4} \\
\hline $08: 15$ & D2.SI.I(I) & $\begin{array}{l}\text { Is relative economy explained by sagittal plane trunk movements in back, } \\
\text { back/front and head loading? } \\
\text { Sean Hudson, Carlton Cooke, Simeon Davies, Sacha-Jane West, Raeeq Gamieldien, Chris Low \& Ray Lloyd }\end{array}$ \\
\hline $08: 23$ & D2.SI.I(2) & $\begin{array}{l}\text { Biomechanical influences in injury rates of United States community rugby-7s: } \\
\text { contact versus non-contact injuries } \\
\text { Victor Lopez Jr, Richard Ma, Meryle G. Weinstein, Patria A. Hume, Robert C. Cantu, Christian Victoria, } \\
\text { Samuel Y. Haleem, Jessica F. Delallo \& Answorth A. Allen }\end{array}$ \\
\hline 08:31 & D2.SI.I(3) & $\begin{array}{l}\text { Pacing strategy, performance parameters and kinematical analysis of club level adolescent } \\
\text { rowers in a simulated rowing ergometer race } \\
\text { Apostolos S. Theodorou, Dimitrios Kourbetis, Eleni Dimakopoulou, Vassilios Panoutsakopoulos, } \\
\text { Barney Wainwright, Sokratis Kaloupsis \& Iraklis A. Kollias }\end{array}$ \\
\hline 08:39 & D2.SI.I(4) & $\begin{array}{l}\text { Optimal timing of low dose caffeine ingestion in trained cyclists } \\
\text { Andrew D. Davenport, Catherine R. Mikus, Benjamin T. Wall, Nima Alamdari \& Francis B. Stephens }\end{array}$ \\
\hline 08:47 & D2.SI.I(5) & $\begin{array}{l}\text { One week L-Arginine supplementation did not improve } 200 \mathrm{~m} \text { swimming } \\
\text { time in trained swimmers } \\
\text { Ozcan Esen \& Ceri Nicholass }\end{array}$ \\
\hline 08:55 & D2.SI.I(6) & $\begin{array}{l}\text { Eccentric hamstring strength: influence on leg stiffness and reactive strength } \\
\text { in elite female youth soccer players } \\
\text { Damian Harper, Dale Forsdyke \& Tom Comyns }\end{array}$ \\
\hline 09:03 & D2.SI.I(7) & $\begin{array}{l}\text { The influence of cold water immersion on markers of recovery following resistance exercise } \\
\text { Laura Wilson, Lygeri Dimitriou, Frank Hills, Marcela Gondek, Emma Cockburn }\end{array}$ \\
\hline
\end{tabular}

Physical Activity For Health

Chair: Dr David Broom FBASES

\begin{tabular}{|c|c|c|}
\hline 08:15 & D2.SI.2(I) & $\begin{array}{l}\text { The validity and reliability of a smartphone pedometer and the effects of the body } \\
\text { characteristics of the user on validity } \\
\text { James Hazelwood }\end{array}$ \\
\hline 08:23 & D2.SI.2(2) & $\begin{array}{l}\text { The effects of pool swimming training on maximal oxygen uptake in recreational } \\
\text { swimming or untrained participants: a systematic review and meta-analysis } \\
\text { lan Lahart \& George Metsios }\end{array}$ \\
\hline 08:31 & D2.SI.2(3) & $\begin{array}{l}\text { An exploration of the meaning of physical activity for working adults } \\
\text { and their reluctance to reduce sedentary behaviour } \\
\text { Kiara Lewis \& Nicola Eccles }\end{array}$ \\
\hline 08:39 & D2.SI.2(4) & $\begin{array}{l}\text { The effects of circuit resistance exercise with added upper quadrant training } \\
\text { on postural sway and functional fitness in older adults } \\
\text { Samuel Oxford, Christopher Ross \& Michael Duncan }\end{array}$ \\
\hline 08:47 & D2.SI.2(5) & $\begin{array}{l}\text { The application of mental skills training for improving health in homeless } \\
\text { young people: a qualitative case study of MST4Life } \\
\text { Benjamin Parry, Sam Cooley, Mary Quinton, Mark Holland, Janice Thompson \& Jennifer Cumming }\end{array}$ \\
\hline 08:55 & D2.SI.2(6) & $\begin{array}{l}\text { Accelerometer based physical activity levels, fundamental movement skills } \\
\text { and weight status in British preschool children } \\
\text { Clare Roscoe, Rob James \& Michael Duncan }\end{array}$ \\
\hline 09:03 & D2.SI.2(7) & $\begin{array}{l}\text { Association of active commuting (walking and cycling) with incident cardiovascular disease, } \\
\text { cancer and mortality: findings from the UK Biobank prospective cohort study } \\
\text { Anne Sillars, Hui Li, Fanny Petermann, Naveed Sattar, Jill Pell, Stuart Gray, Jason Gill \& Carlos Celis-Morales }\end{array}$ \\
\hline
\end{tabular}

Psychology I

Chair: Prof Chris Harwood FBASES

08:15-09:10, D2.S1.3. Conference Theatre

\begin{tabular}{l|l|l|l|l}
\hline $08: 15$ & D2.SI.3(I) Linking coaches' recovery, well-being and athletic performance: a day-level study on
\end{tabular} antecedents and consequences of autonomy support in elite sport Yannick Balk, Jan de Jonge, Wido Oerlemans \& Sabine Geurts 


\begin{tabular}{|l|l|l|}
\hline $\mathbf{0 8 : 2 3}$ & D2.SI.3(2) & $\begin{array}{l}\text { Can young athletes differentiate between conditional and unconditional social influence } \\
\text { from significant others? An answer from exploratory structural equation modeling } \\
\text { Derwin King Chung Chan }\end{array}$ \\
\hline $\mathbf{0 8 : 3 1}$ & D2.SI.3(3) & $\begin{array}{l}\text { Situational antecedents of outward emotional reactions in table tennis } \\
\text { Julian Fritsch, Diana Zerdila, Anne-Marie Elbe, Antonis Hatzigeorgiadis }\end{array}$ \\
\hline $\mathbf{0 8 : 3 9}$ & $\mathbf{D 2 . S 4 . 4 ( 4 )}$ & $\begin{array}{l}\text { Two case studies of decision-making under stress } \\
\text { Anne-Claire Macquet \& Héloïse Lacouchie }\end{array}$ \\
\hline $\mathbf{0 8 : 4 7}$ & $\mathbf{D 2 . S 4 . 4 ( 5 )}$ & $\begin{array}{l}\text { The relationship between personality, gender, mental toughness } \\
\text { and athletic coping skills in fencers } \\
\text { Gerard Madden \& Mike Westgate }\end{array}$ \\
\hline $\mathbf{0 8 : 5 5}$ & D2.S4.4(6) & $\begin{array}{l}\text { Psychological resilience, challenge and threat states, and sports performance under pressure } \\
\text { Lee Moore, Tom Young \& Mustafa Sarkar }\end{array}$ \\
\hline $\mathbf{0 9 : 0 3}$ & D2.S4.4(7) & $\begin{array}{l}\text { The relationship between anxiety and depression symptoms and help-seeking intentions } \\
\text { in individual sport athletes and university students: the moderating role of gender and } \\
\text { participant status } \\
\text { Richard Taehtinen \& Hafrun Kristjansdottir }\end{array}$ \\
\hline
\end{tabular}

\section{Psychology 2}

Chair: Lina Vaisetaite (FEPSAC)

08:15-09:10, D2.S1.4. Suite 3

\begin{tabular}{|l|l|l|}
\hline 08:15 & D2.SI.4(I) & $\begin{array}{l}\text { Examination of factors influencing sport-enjoyment in physical education in adolescents } \\
\text { Eliane Stephanie Engels, Philipp Alexander Freund }\end{array}$ \\
\hline $\mathbf{0 8 : 2 3}$ & D2.SI.4(2) & $\begin{array}{l}\text { A case study focusing on the delivery and evaluation of a motivation } \\
\text { programme for youth sport coaches } \\
\text { Helen Ferguson, Michael Noetel, Chris Lonsdale }\end{array}$ \\
\hline $\mathbf{0 8 : 3 1}$ & D2.SI.4(3) & $\begin{array}{l}\text { Methodology goes wild: advocating the advancement of cognitive ethnography } \\
\text { in physical activity research } \\
\text { Danny Golding, Gail Kinman \& Steve Kozub }\end{array}$ \\
\hline $\mathbf{0 8 : 3 9}$ & D2.SI.4(4) & $\begin{array}{l}\text { Mental skills support within elite professional tennis: Reflections on a 5-year } \\
\text { programme of delivery } \\
\text { John Mathers }\end{array}$ \\
\hline $\mathbf{0 8 : 4 7}$ & D2.SI.4(5) & $\begin{array}{l}\text { The effects of achievement goals and motivational context on psychological functioning } \\
\text { and sport performance: An experimental investigation } \\
\text { Mairi Mulvenna, James W. Adie, Luke Sage, Nigel Wilson \& Douglas Howat }\end{array}$ \\
\hline $\mathbf{0 8 : 5 5}$ & D2.SI.4(4) & $\begin{array}{l}\text { A neophyte practitioner's reflections on providing sport psychology support } \\
\text { to an injured footballer } \\
\text { Rachael Newport, David Shearer, Camilla Knight }\end{array}$ \\
\hline $\mathbf{0 9 : 0 3}$ & D2.SI.4(5) & $\begin{array}{l}\text { Student-athlete academic performance at university: a mixed-method case study } \\
\text { Olivier Rouquette, Philippe Godin, Guy Lories, Anne-Sophie Leurquin }\end{array}$ \\
\hline
\end{tabular}

Sport and Performance

Chair: Adam Grainger

08:I5-09:I0, D2.SI.5. Suite I

\begin{tabular}{|c|c|c|}
\hline $08: 15$ & D2.SI.5(I) & $\begin{array}{l}\text { Implicit and explicit coordination mechanisms in youth football: temporal adaptations } \\
\text { in team cognition and correlations to team performance } \\
\text { Marc Blaser \& Roland Seiler }\end{array}$ \\
\hline $08: 23$ & D2.SI.5(2) & $\begin{array}{l}\text { Breast support implications for an elite female rower: a multi-disciplinary case study } \\
\text { Jenny Burbage, Mitch Lomax \& Chris Mills }\end{array}$ \\
\hline 08:31 & D2.SI.5(3) & $\begin{array}{l}\text { Interday reliability and usefulness of reactive strength index derived } \\
\text { from the ten to five repeated jump test } \\
\text { Thomas Comyns, Eamonn Flanagan, Damian Harper, Sean Fleming \& Evan Fitzgerald }\end{array}$ \\
\hline 08:39 & D2.SI.5(4) & $\begin{array}{l}\text { A comparison of the hormonal and performance profiles of male Greco-Roman and Freestyle } \\
\text { wrestlers during maximal exercise testing } \\
\text { Blair Crewther, Zbigniew Obminski \& Lech Borkowski }\end{array}$ \\
\hline $08: 47$ & D2.SI.5(5) & $\begin{array}{l}\text { Influence of stochastic movement patterns on external and internal load measures during } \\
\text { simulated rugby league match play } \\
\text { Thomas Mullen, Craig Twist \& Jamie Highton }\end{array}$ \\
\hline $08: 55$ & D2.SI.5(6) & $\begin{array}{l}\text { The reliability of integrated external:internal load ratios for field based } \\
\text { measures of fitness in rugby union } \\
\text { Richard Taylor, Dajo Sanders, Tony Myers \& Ibrahim Akubat }\end{array}$ \\
\hline 09:03 & D2.SI.5(6) & $\begin{array}{l}\text { The effects of } \boldsymbol{\beta} \text {-alanine on indoor bouldering performance } \\
\text { Daniel Wood }\end{array}$ \\
\hline
\end{tabular}



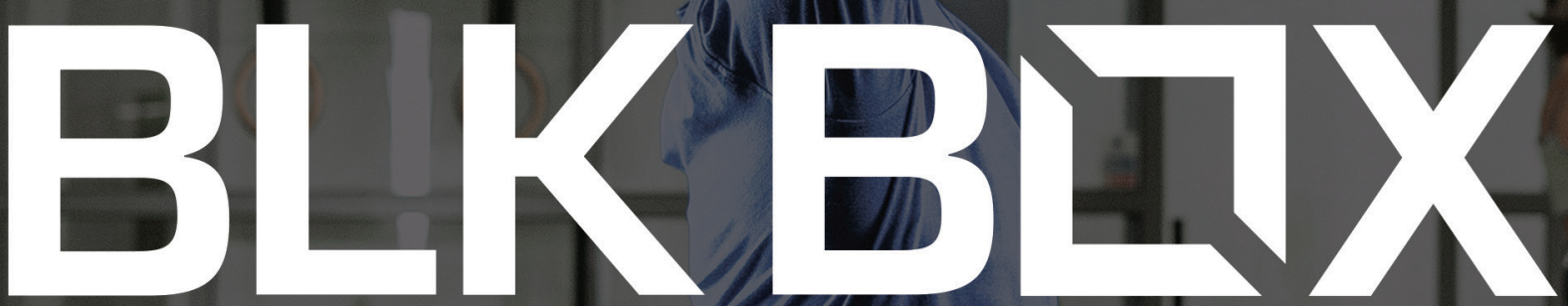

(e)

Performance Equipment \& Facility Design

\section{WWW.BLKBOXFITNESS.COM}




\section{renew ${ }^{0}$ \\ Enhanced Circulation}

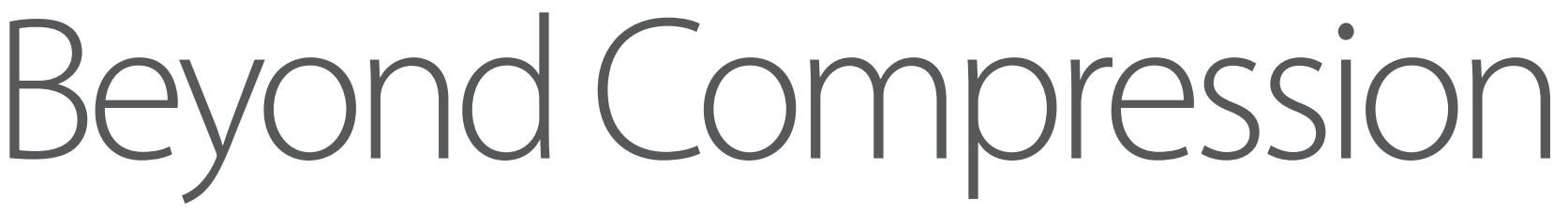

Introducing Renew Therapy, a non-invasive

treatment using the latest Renew ECP technology.

The physiological effects stimulated by Renew ECP Therapy

can deliver benefits to athletes looking to aid recovery

and enhance sports performance.

The ECG-optimised compression treatment has been shown to substantially reduce the levels of stress and inflammation markers, further aiding an accelerated recovery time.

For more information call 08000988220

or email us at: info@renewgroup.eu

Visit our new website at: www.renewtherapy.co.uk

(f) (웅 


\section{Getting around the East Midlands Conference Centre}

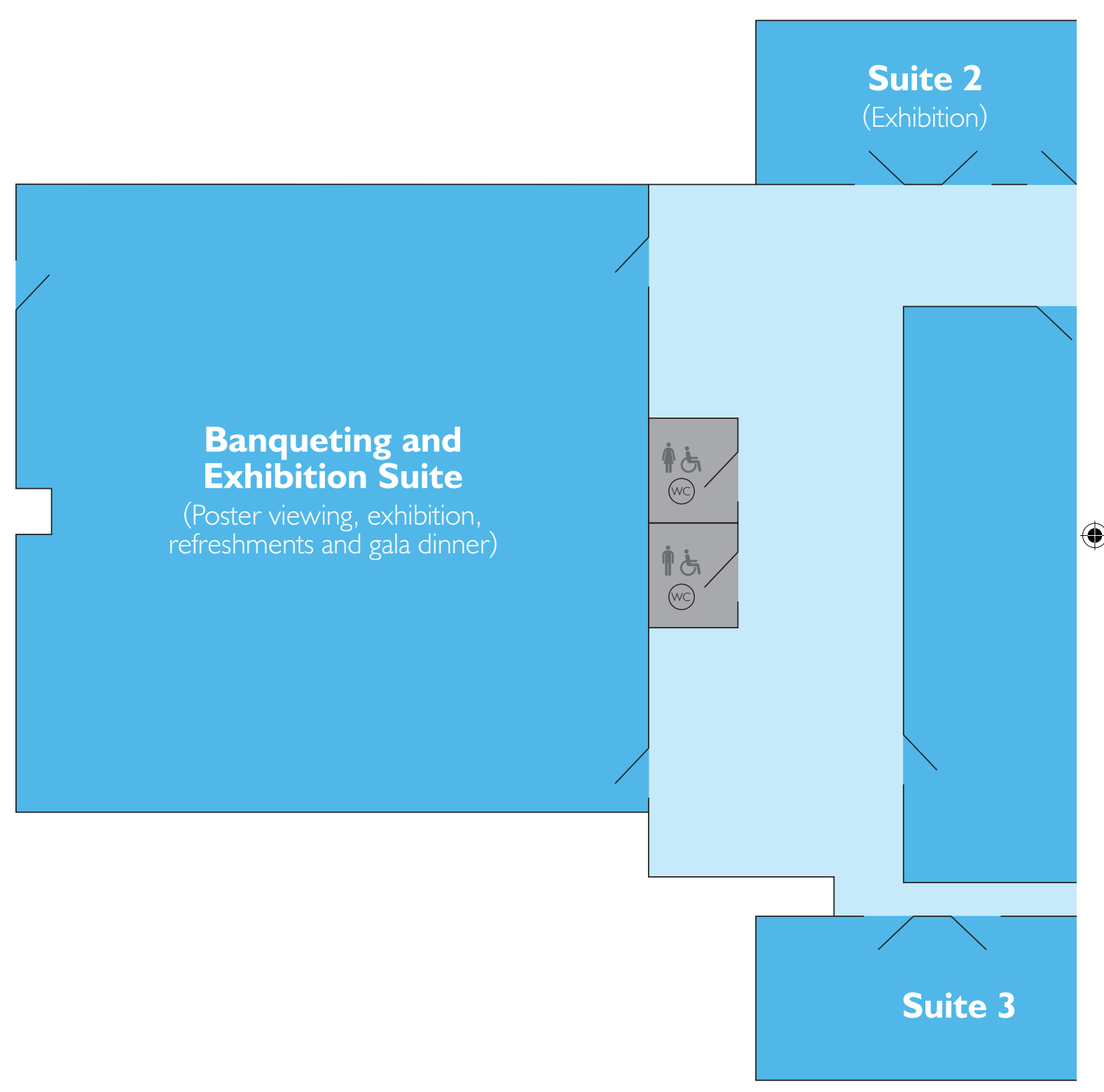



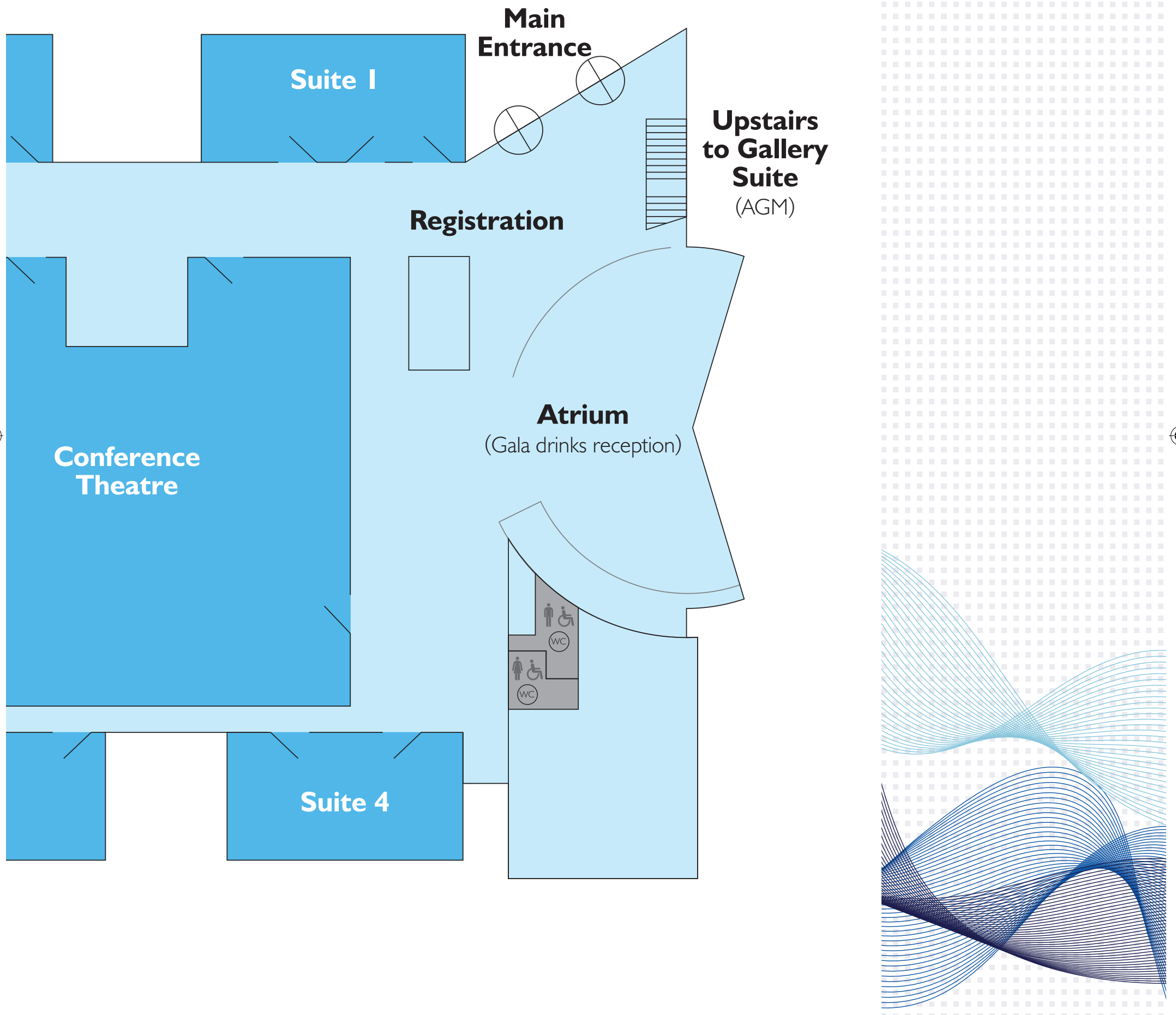


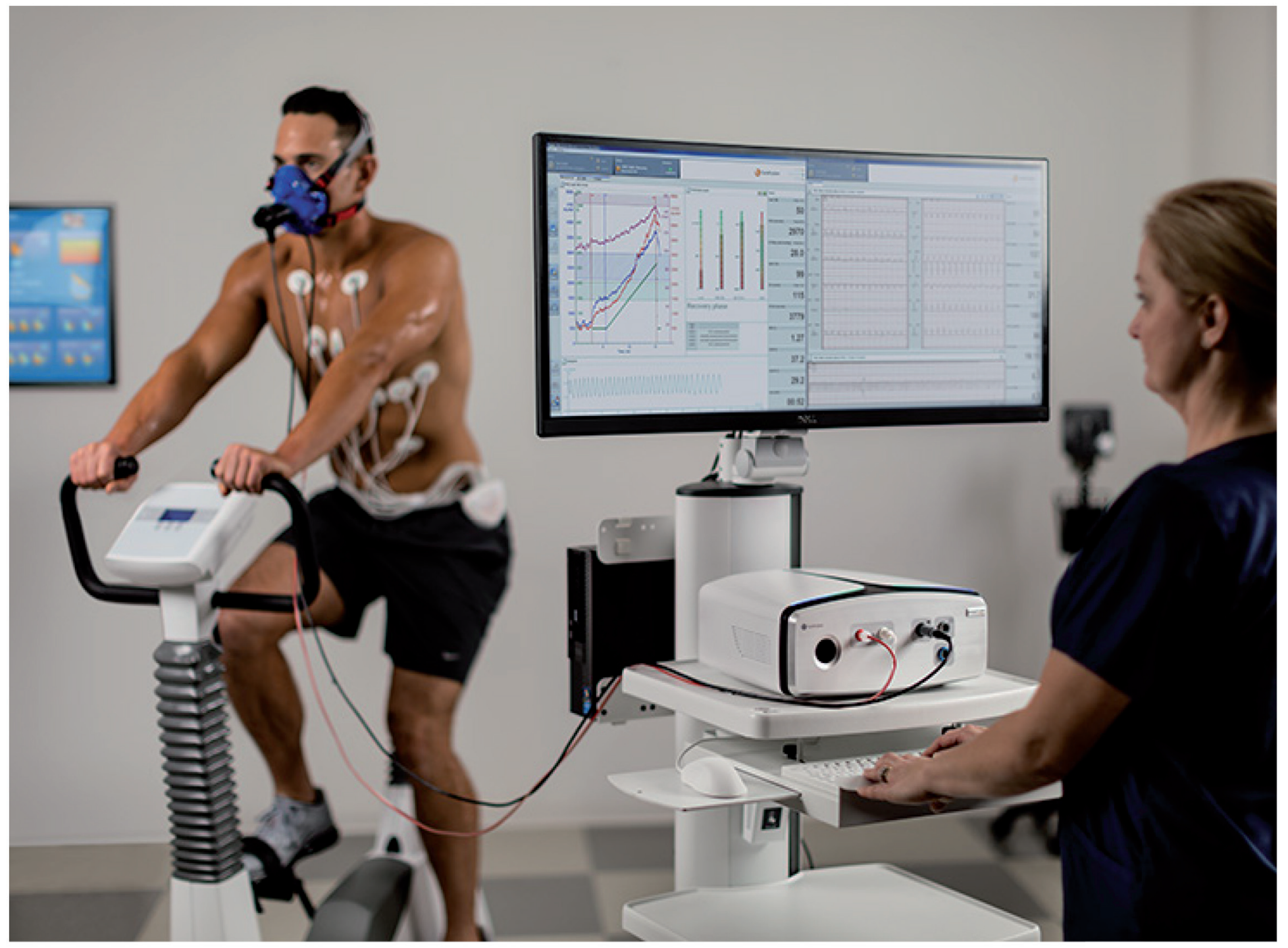

$\oplus$

\section{Vyntus ${ }^{\circledR} \mathrm{CPX}$ \\ The legend continues.}

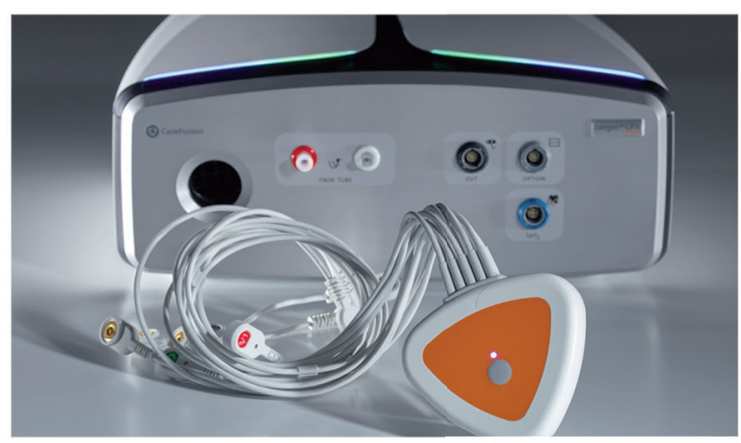

\section{ACCURATE -}

Trusted and improved high-end sensor technology from well-known Oxycon Pro with additional internal drying mechanism - ideal for long term measurements in sports medicine.

\section{FLEXIBLE -}

From clinical patients to high-performance athletes in breath by breath mode. Optional $\mathrm{High} / \mathrm{Low} \mathrm{FIO}_{2}$ measurements up to $\mathbf{1 0 0} \% \mathrm{O}_{2}$ and optional canopy method for indirect calorimetry with outstanding hygiene concept.

\section{HELPFUL -}

Easy to use SentrySuite ${ }^{\circledR}$ Software with customizable evaluation workflow, three ventilatory thresholds, automatic slope calculations, fully integrated Vyntus ${ }^{\circledR}$ ECG using Bluetooth ${ }^{\circledast}$ technology and many more.

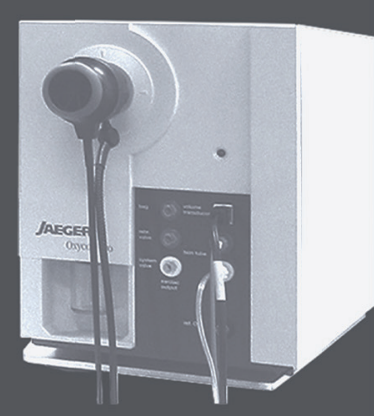

() 2016 CareFusion Corporation or one of its affiliates. All rights reserved.

CareFusion Germany $234 \mathrm{GmbH}$ is a Bluetooth SIG member. Vyntus and SentrySuite are trademarks or registered trademarks of CareFusion Corporation or one of its affiliates. All trademarks are property of their respective owners. 


\section{Delivering Best in Class Products that Perform}

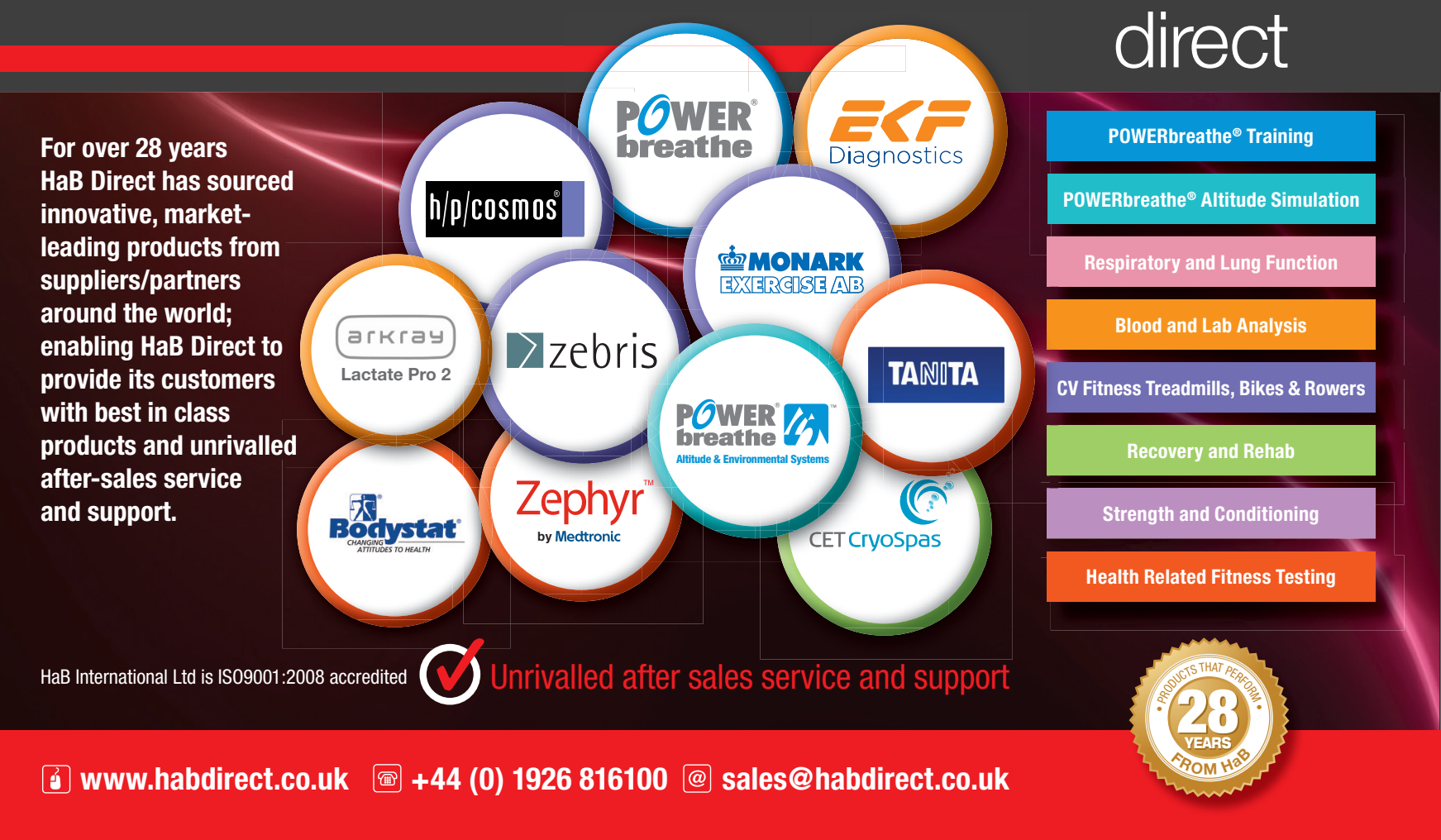

BoB (Biomechanics of Bodies) is a musculoskeletal modelling system, developed in the MATIAB environment, which consists of a human skeleton, the joints connecting the bones and over 600 of the major locomotor muscles. BoB is ideally suited for sports' analysis, easy to use for teaching and has advanced features for research.

BoB is a standalone package which can calculate anatomical trajectories, muscle loads, joint contact forces, ground reaction forces, energy/power expenditure for individual muscles, a muscle editor and a graphics engine to display multi-subject graphics as images, videos and tabulated output. BoB can read motion and force data from c3d, Xsens, Perception Neuron, csv and MATIABB. Output is MATIAB compatible.

For further information on $\mathrm{BoB}$, examples of applications and to download a trial version visit: BoB-biomechanics.com or see: youtube.com/mendip89

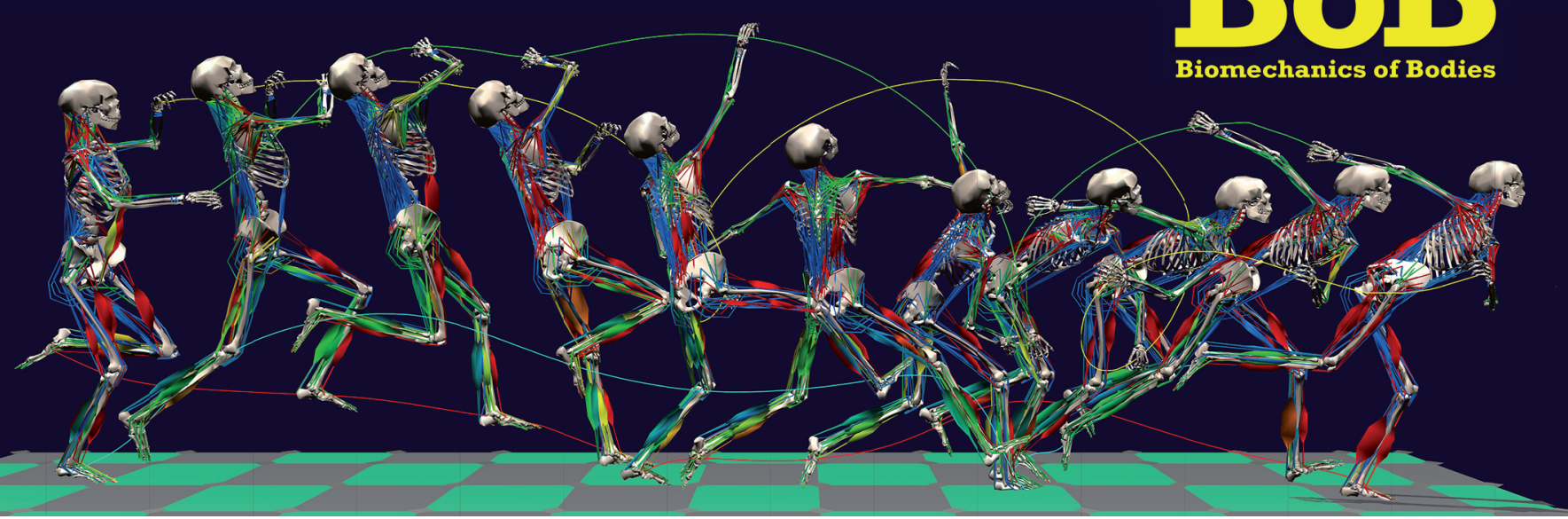




\section{Supporter and exhibitor information}

The British Association of Sport and Exercise Sciences (BASES) is dedicated to promoting excellence in sport and exercise sciences. BASES members enjoy a wide range of CPD opportunities through workshops, webinars and conferences and are able to obtain professional recognition through BASES accreditation programmes and Chartered Scientist status. Members can keep up-to-date with the latest news, research and career opportunities via monthly e-newsletters and our quarterly publication The Sport and Exercise Scientist. Build your network, enhance your CV, open doors and further your career by becoming a BASES member to gain access to the largest sport and exercise science network in the UK.

www.bases.org.uk

\section{Platinum supporter: BLK BOX}

At BLK BOX we are bold and ambitious. We know what works and more importantly, we know what doesn't. We are one of few companies in the UK able to provide complete turnkey fitness solutions for the Sports Performance market. All of which are designed and shipped directly from our brand new 20,000sq ft manufacture, warehousing and education facility located in the heart of Belfast. The BLK BOX methodology is to ensure that we are creating optimal training space for clients and providing the best equipment to serve their training needs. We don't believe in compromise and you shouldn't either. We have a commitment to all our clients to ensure they get the best possible solution and we hope we can share this with you. The market has evolved and it is more competitive than ever. When you work with BLK BOX we like to know everything about your business and your plans. We take a simple approach to business. You succeed, we succeed. We take a personal interest in your business to ensure your venture is as successful as possible. We don't compromise in business or training and we don't think you should either.

www.blkboxfitness.com

Platinum supporter: Renew Health Limited

Renew Therapy gives everyone the opportunity to enhance their circulation through the introduction of ECP Therapy. Widely regarded as an effective method to increase blood flow, ECP Therapy can aid in improving quality of life, cardiovascular efficiency and sports recovery. During treatment, inflatable cuffs are wrapped around the lower body. ECG sensors are then placed on the chest to detect the heartbeat. The inflation of the cuffs, in time with each heartbeat, compresses blood from the lower legs back to the heart, improving blood flow in and around the heart. The benefits of this treatment have clinically shown to provide improvement in vasodilation, increased VO2 and increased blood flow. The concept of obtaining a full cardiovascular workout without the physical stress of training or 'No Intensity Training' can help everyone, from athletes who are unable to train, but still maintain a high level of cardio fitness, to individuals who are inactive and seeking a to way improve their energy levels and lead a more active life. Renew ECP Therapy is part of Renew Health Ltd, established in Europe to develop and distribute innovative, life-enhancing technologies. Our vision is to deliver innovation, making a difference to mankind.

info@renewgroup.eu

\section{Gold supporter: Cyclus2}

With over 40 years' experience, Cyclus 2 has established a new gold standard in performance \& clinical research for universities and professional cycling teams. With a slip-free resistance of $3000 \mathrm{~W}$, the use of the athlete's own bike together with the elastic suspension system, Cyclus 2 offers a distinct advantage over other common ergometers, providing athletes with a
The European Federation of Sport Psychology (FEPSAC) aims at supporting the development of sport psychology in Europe by promoting scientific, educational and professional work; disseminating information and promoting cooperation; and maintaining social and scientific relations between groups working in sport psychology. FEPSAC members benefit from participation in congresses, access to the official journal Psychology of Sport and Exercise, staying up-to-date with the latest sport psychology news (e.g. through a monthly Newsflash) and the possibility to disseminate events via our website and social media. Join the largest sport psychology organization in Europe and help us to develop and promote our field.

www.fepsac.com

real-world cycling experience \& researchers with ecologically enhanced results. Standard performance tests are included in the software - new tests can easily be programmed. Testing and training modes include power, torque, isokinetic and simulation. Packed with unique technology and compatible with software platforms (i.e TrainingPeaks/ VirtualTraining), the ergometer is extremely versatile, mobile and adaptable to all common cycling frames (including children frames). Cyclus 2 also produces specialised ergometers for track cyclists, handbikes and also eccentric ergometers. Cyclus 2 ergometers are exclusively distributed by Advanced Cycling Science in the UK and Ireland.

www.cyclus2.com

www.advancedcyclingscience.co.uk

\section{Gold supporter: Routledge}

Routledge publishes journals across the full span of the sport discipline, including sport and exercise science, sport medicine, physical education, sport coaching, sport management, sport development, sport technology and engineering, research methods in sport, and the history, politics, sociology and philosophy of sport. This cross-disciplinary collection enables us to reach the widest possible audience within sport and exercise studies. We are also well-established in the related areas of medicine, biomechanics, ergonomics and health. Over the last fifteen years, Routledge's list of sport and exercise books has grown into one of the largest and broadest of any major international academic publisher, with around I,000 books in print in sport, exercise, and related subject areas. Our portfolio includes books at all levels, from market-leading undergraduate textbooks and books for practitioners and professionals, to cutting-edge research monographs and reference books for the international academy.

www.tandfonline.com/toc/rjsp20/current

Gold supporter: Wattbike

Wattbike is the ultimate indoor bike. Wattbike remains unrivalled in cycling training, performance and data. Born out of an obsession with performance and developed in partnership with British Cycling, Wattbike has been at the forefront of cycling technology and training methodology from the very start. Working with the world's best sport scientists and engineers, Wattbike shape data to help deliver actionable insights to riders in real time, including a unique and patented Polar View technology. Combined with a fully customisable ride setup and the feel of riding a real bike, it provides the user with a highly effective and efficient power-based training solution. Wattbike isn't just the choice of elite road and track cyclists. World and Olympic champions across all sports use Wattbike to train smarter, push harder and improve performance and it is ingrained in elite sport at the highest level around the world.

www.wattbike.com 
BIOPAC Systems Inc. are a leading manufacturer of Data Acquisition Systems for sports science \& exercise physiology research and teaching. We provide wired and wireless systems for examination of parameters including EMG, ECG, ventilation, gas analysis, continuous non-invasive blood pressure and cognitive function. Systems also safely and easily interface with dynamometers, ergometers, force plates, load cells and other existing apparatus.

www.biopac.com

BoB Biomechanics will be demonstrating $\mathrm{BoB}$ - the musculoskeletal modelling system. It's easy to use for the analysis of sports performance and robust enough for teaching or research. BoB contains a skeletal model, over 600 locomotor muscles, an inverse dynamics solver, a muscle editor and a powerful graphics engine. Outputs include muscle force distribution, joint contact forces, ground reactions, anatomical trajectories and many others. BoB has interfaces to MATLAB, c3d, Xsens, Perception Neuron and csv.

www.BoB-biomechanics.com

COSMED are world leading suppliers of Cardio Pulmonary, Metabolic testing and Body Composition solutions to sports science, health, professional sport and wellness. COSMED products on show during BASES 2017 include: K5, metabolic system for field and laboratory testing; Quark CPET, fully-featured and highly accurate metabolic cart; Fitmate PRO, desktop device for new approach to VO2max and REE testing; BOD POD, the practical Gold Standard body composition solution in adults and children, based on air displacement plethysmography technology.

www.cosmed.com

Cranlea Human Performance Ltd are delighted to be once again exhibiting at the annual BASES conference. Cranlea Human Performance stands for providing the best equipment at affordable prices, delivery quality and service every time. Come and speak to us about the latest innovations from Woodway, Cortex and Lode along with exciting developments from our other suppliers. If you haven't already done so, check out our new website at www.cranlea.co.uk and let us know what you think. Enjoy the conference.

www.cranlea.co.uk

Firstbeat is the leading provider of physiological analytics for sports, fitness, performance and wellbeing. Firstbeat facilitates better training optimization, reduces injury risks, and helps fast-track player development. Over 700 elite sports teams in top leagues around the world rely on Firstbeat to take the guesswork out of coaching decisions, while academics use our HRV analytics to enhance their research and understanding of human performance.

www.firstbeat.com

HaB Direct has exclusive brands, which include: POWERbreathe K5 with Breathe-Link - Time Efficient, Performance Training, Testing and Monitoring device; POWERbreathe Altitude Systems (PBAS) comprehensive, versatile and affordable product range available, $\mathrm{h} / \mathrm{p} /$ cosmos treadmills; Monark cycle - upper and lower body cycle ergometers for testing, performance training and rehab; MARPO Rope Trainers; and Zephyr Performance Systems. Other exclusive brands include: EKF - Lacate Scout + Biosen; Zebris; Lactate Pro and Tanita supported by industry leading customer support.

www.habdirect.co.uk

At Human Kinetics our mission is to produce innovative, informative products in all areas of physical activity that helps people worldwide lead healthier, more active lives. We are committed to providing quality information and educational products in physical activity and health fields that meet the needs of our customers.

www.humankinetics.com/europe

Kistler Instruments Ltd will be showcasing our force plates together with Kistler MARS software; a versatile tool for performance assessment in sports and rehabilitation. It is designed to provide all relevant performance data to help optimise athlete development, prevent injury and monitor fatigue. It supports frequent testing in a very short amount of time and gives a clear overview of the parameters that matter most, helping to set clear developmental goals.

www.kistler.com/gb/en/

Lake Image Systems Ltd is the exclusive UK distributors for Fastec, AOS and Xcitex High Speed Camera Systems and Analysis Software. Used in many sports development applications, this technology can be used to improve performance, rehabilitation, biomechanics research and equipment analysis. Systems can feature battery power options and long record times, while comprehensive software allows for quick and easy motion tracking, measurement and analysis.

www.lakeimage.co.uk
At Medical Graphics UK Ltd we understand that leading edge technology produces the most accurate results in research and performance management. That's why our highly dependable Cardio-Respiratory equipment and industry-leading support services are being chosen ever more by top Universities, Research Facilities and Sports Science Institutions to ensure optimum results, performance and minimum downtime. www.medicalgraphicsuk.com

Perform Better is the UK's most innovative supplier of Performance Monitoring and Training Equipment specialising in the Elite Professional Sports and Education markets. We are committed to providing the latest and most cutting edge equipment, constantly updating our product range to offer our customers the latest technologies from around the World. Look out for some key products on our stand including: Gymaware, ForceDecks, Optojump, Witty Timing System, Witty SEM, Hamstring Solo, Myontec and Catapult, amongst others.

www.performbetter.co.uk

SMART Medical provide innovative cardio/neurovascular diagnostic systems including the Finapress NOVA - the comprehensive solution continuous NiBP monitoring \& Cardiscope/Mega - heart rate variability analysis for assessment of autonomic function, sleep analysis \& sports optimisation utilising "bio-patch" recorders for long-term and realtime measurements. DWL TCD systems with analog $\mathrm{l} / \mathrm{O}$ provide a comprehensive research bridge for up to 8 channels, whilst SphygmoCor \& Vicorder PWA/PWV provide CBP and non-invasive haemodynamic monitoring solutions.

www.smartmedical.co.uk

Sporting Edge (UK) Ltd is a UK based company with an expanding global reputation as the leader in the design and provision of Altitude and Environmental Facilities. Formed in 2005, Sporting Edge has consistently set the benchmark for performance, safety, reliability and control regarding Simulated Altitude, Temperature and Relative Humidity. Endorsed by the English Institute of Sport and a client list that includes many UK Universities, Sports Teams and Health and Fitness clubs.

www.trainingwithaltitude.co.uk

Tanita is the global health monitoring leader thanks to the company's cutting-edge medical level BIA technology. Tanita body composition monitors today are vital measuring and monitoring tools in fitness, health and leisure centers, sport clubs and leading hospitals. Designed and engineered to provide an instant assessment of an individual's health and fitness status, Tanita body composition underline the professional approach and effectiveness of your health and fitness programs. Tanita body composition monitors help boost client motivation and are a superior way to retain clients.

www.tanita.eu

Tracksys Ltd provides integrated solutions and services for behavioural research within the UK and Ireland. Key products available on our stand to discuss and try out include: Noldus FaceReader - automatic analysis of facial expressions; Noldus Observer XT - behavioural scoring and integration system; Labs - fixed and portable; Mindware - psychophysiology solutions SIMI - motion analysis tools; and SMI Eye Trackers - glasses and portable. non-contact eye trackers.

www.tracksys.co.uk

UCLAN is one of the UK's top providers of sport, outdoor and wellbeing education and our global reputation grows by the day. Our sports, exercise and nutrition degrees are informed by our world-leading research and taught by internationally-acclaimed academics. Our courses are delivered from multi-million pound, world-class facilities in Preston including purposebuilt classrooms, laboratories, Sir Tom Finney Sports Centre and our Sports Arena, where you will develop the skills and knowledge you need to excel. www.uclan.ac.uk/schools/sport-wellbeing/courses.php

Vyaire Medical (formerly CareFusion) is a global leader in Cardiopulmonary Exercise Testing. Vyaire Respiratory Diagnostics is a committed and passionate organisation focused on manufacturing high quality CPET equipment and connectivity solutions. With a proven track record and over 60 years of continuous development and innovation, Vyaire remains at the forefront of Cardiopulmonary Exercise Testing. Our iconic brands include; Jaeger Oxycon Pro, MasterScreen CPX and Vmax Encore and more recently our Vyntus CPX system."

www.vyaire.com

Wolters Kluwer will be showcasing our key new and best-selling sports science titles at BASES, which will include the new edition of ACSM's Guidelines for Exercise Testing and Prescription, ACSM's Health-Related Physical Fitness Assessment Manual and McArdle's Essentials of Exercise Physiology. Come to our stand to learn more about these titles, arrange inspection copies and find out about our latest offerings.

www.lww.co.uk 

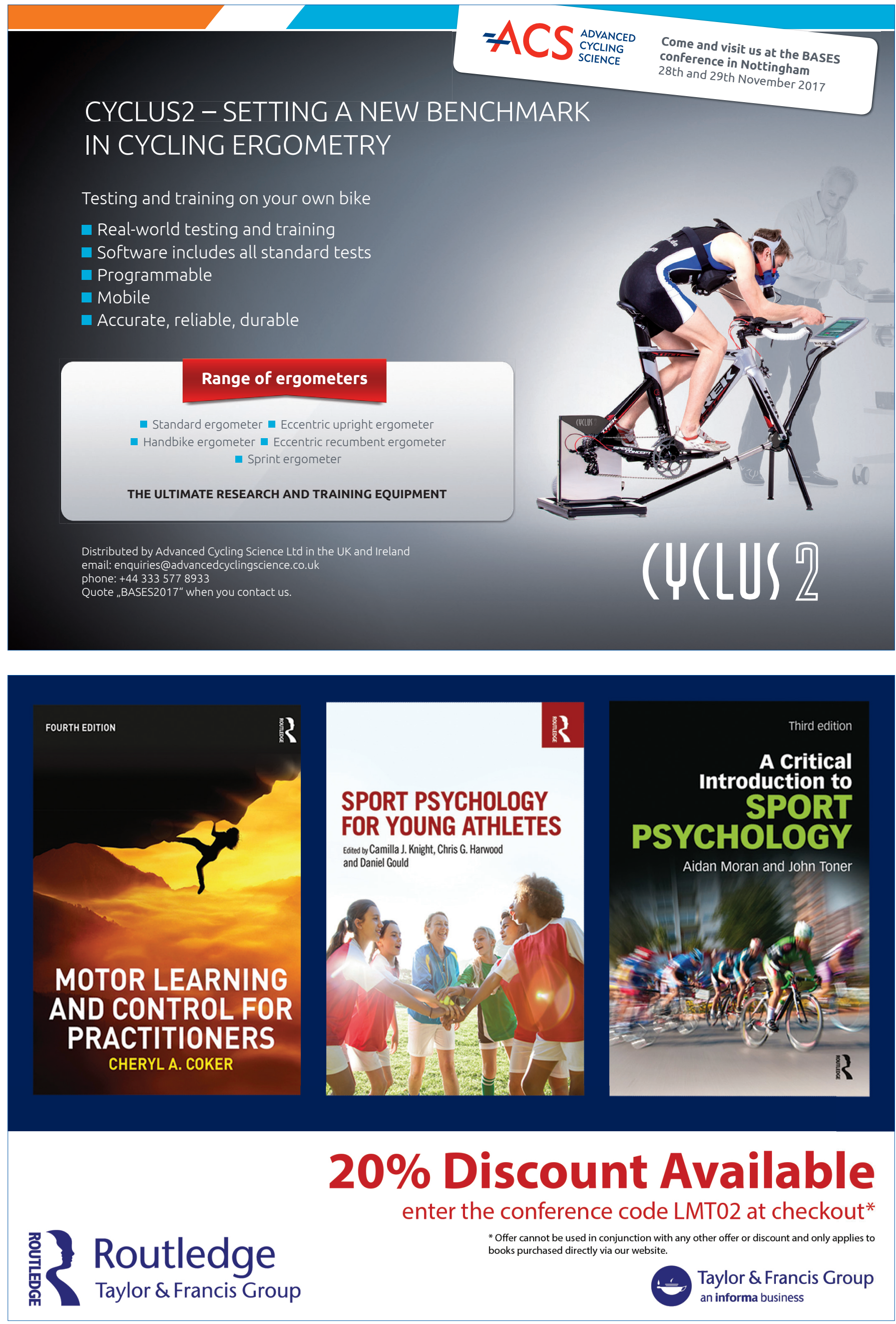
wattbike

\section{ACCURATE, RELIABLE, REPEATABLE DATA}

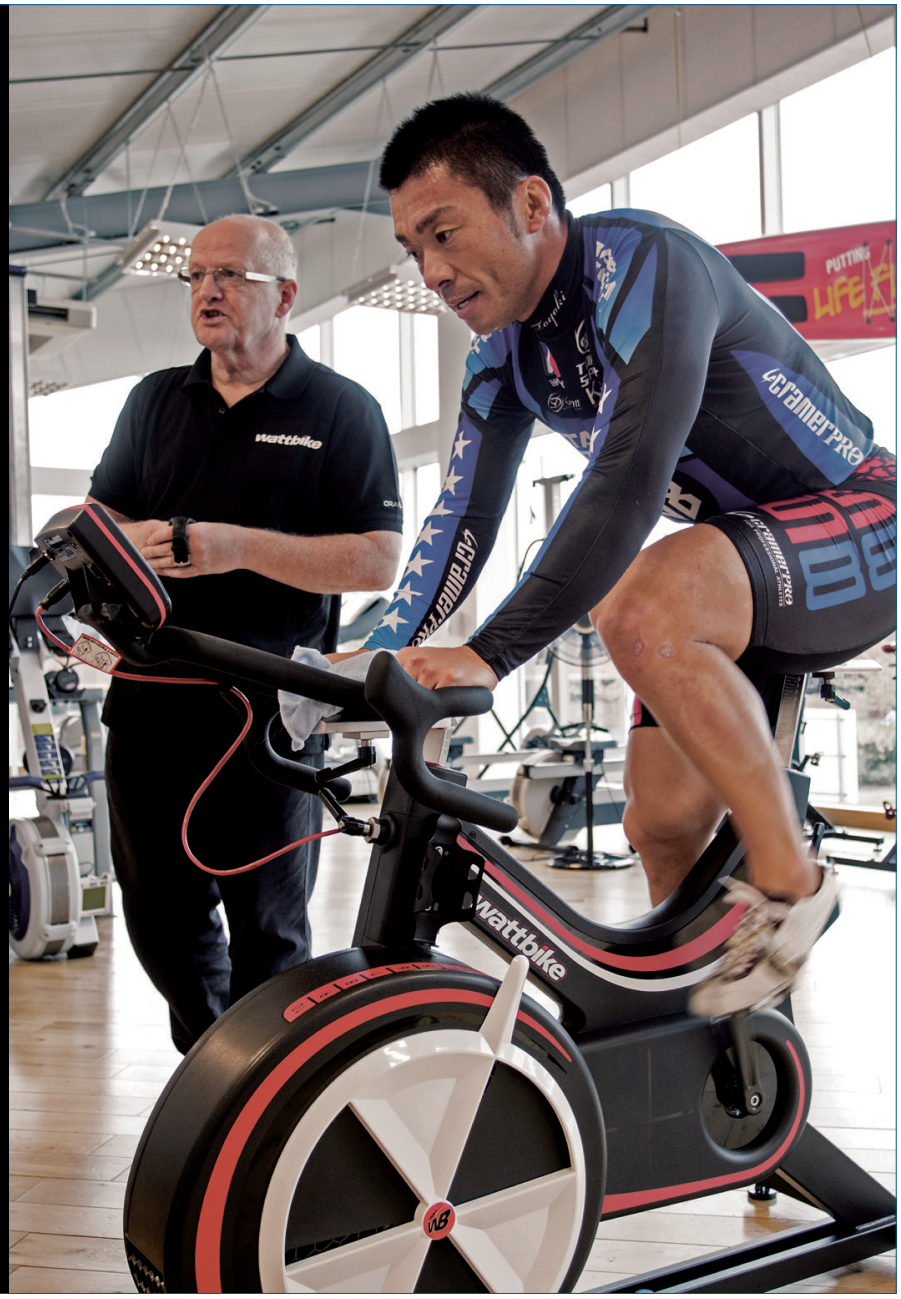

WATTBIKE . COM

Y @Wattbike f /Wattbike Voun /Wattbike @ /Wattbike

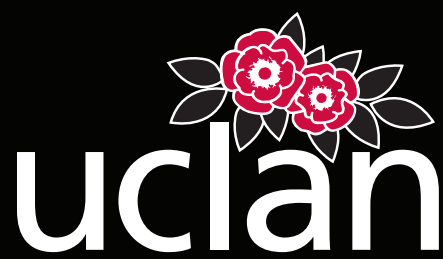

University of Central Lancashire

- PRESTON -

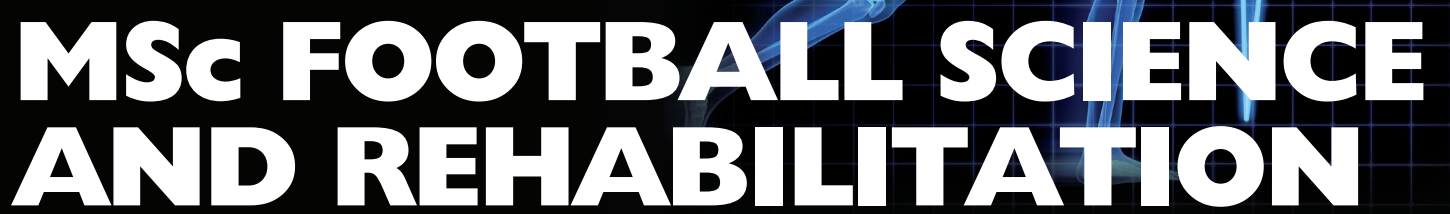

This part-time course presents a range of options for study from a Postgraduate Certificate to a full Master of Science programme. The course is suitable for those wishing to enhance their skills and knowledge in various areas including Sports \& Exercise Science, Physiotherapy and Sports Therapy.

The course covers performance, conditioning, injury rehabilitation and prevention, as well as analytics and a scientific approach to football practice.

Study in short intensive blocks with some distance delivery.
FOR FURTHER INFORMATION please cONTACT

Mark Leather (Course Leader): MLeather2@uclan.ac.uk

Course Enquiries: cenquiries@uclan.ac.uk - 01772892400

www.uclan.ac.uk 


\title{
A to $\mathbf{Z}$ help guide
}

\author{
- Annual General Meeting (AGM). \\ The BASES Annual General meeting will take place on Tuesday 28 November at 17.30-18.30 at East \\ Midlands Conference Centre in Suite I.
}

The FEPSAC Annual General meeting will take place on Tuesday 28 November at 17.30-18.30 at East Midlands Conference Centre in the Gallery Suite.

- Awards. A total of ten prestigious awards are available at this year's conference. The BASES and FEPSAC Conference awards seek to reward outstanding contributions to sport and exercise sciences by BASES and FEPSAC members.

\section{- BASES Awards.}

- Human Kinetics Student Free Communication Presentation Award One award of $£ 100$ Human Kinetics book vouchers for the best student free communication presentation made by a BASES student or graduate member.

- Human Kinetics Student Poster Presentation Award One award of $£ 100$ Human Kinetics book vouchers for the best student poster presentation made by a BASES student or graduate member.

- Sportesse Sport and Exercise Science Free Communication Presentation Award A $£ 500$ cash prize for the best free communication presentation on sport and exercise science made by a BASES professional member.

- Routledge Recently Qualified Researcher Free Communication Presentation Award A prize of $E 500$ (half cash/half book tokens) for the best free communication presentation given by a recently qualified researcher (BASES member).

- BASES Sport and Exercise Science 5 Slides in 5 Minutes Free Communication Presentation Award

One award of $£ 500$ (half cash/half BASES vouchers) for the best 5 slides in 5 minutes free communication presentation on sport and exercise science by a BASES professional member.

\section{- Cranlea Poster Presentation Award}

One award of a Polar Heart Rate Monitor for the best poster presentation made by a BASES member.

- Routledge/Taylor \& Francis Sport and Exercise Science Impact Award One award of $£ 500$ (half cash/half book tokens) for the best free communication presentation demonstrating outstanding impacts in terms of reach and significance made by a BASES or FEPSAC member.

\section{- FEPSAC Awards.}

- FEPSAC/Elsevier Poster Award for Young Researchers in Sport and Exercise Psychology Three awards (cash prizes of $€ 500, € 300$ and $€ 200$ ) for the best three poster presentations made by FEPSAC student members.

- BASES Fellowships. Individuals that have been awarded BASES Fellowships this year will be recognised at the gala dinner. The award recognises esteemed professional achievement, skills, knowledge and service to BASES and the sport and exercise science community.

- Car parking. There is complimentary parking in the EMCC/Hotel car park with no permit required. If you do not park in this car park and park anywhere else on Campus you must display a parking permit which can be collected free of charge from the EMCC or Hotel reception. If a permit is not displayed you will receive a parking fine.

- Certificates of attendance. These will be e-mailed to delegates post-event following completion of the conference evaluation.

- Cash and payment. EMCC has a cash machine (charges a fee) and there are also bank facilities and cash machines available in the Portland Building on campus.

- Check in and check out. The Orchard Hotel operates a 14:00 check in and I I:00 check out.

- Cloakroom and left luggage. There will be a cloakroom available at EMCC. Please note this cloakroom will not be staffed so valuable items should not be left here. Neither BASES, KC Jones nor EMCC can accept responsibility for the loss of personal property at this conference. 
- First aid. The venue have qualified first aid staff members on hand to deal with emergencies. Further assistance (including contacting the emergency services) will be provided.

- Gym facilities. The Orchard Hotel has a very small gym in the basement which is open from 06:00 until 22:00. The main facilities of the hotel are from 07.30-22.00. Delegates can use the University gym which is across the road from the EMCC and the guests just need to take the keycard and wallet to gain access. The opening times are Monday 07.30-22.30, Tuesday - Friday 07.15-15.00.

- Help desk. If you require assistance or information you will be able to find a member of the conference team on the registration desk or at the BASES stand located in the Banqueting Suite who will be happy to help.

- Internet/Wi-Fi access. Log into 'DeVere Eastmidlands Conference' Wi-fi. No password is required, please just accept the terms and conditions from the welcome screen.

- Journal of Sports Sciences. All BASES Conference 2017 abstracts will be published in an online supplement of the Journal of Sports Sciences. Free open access is available until 3I December at www.tandfonline.com/risp. From I January 2018 onwards BASES members can gain online access to the supplement and their issues of the Journal of Sports Sciences by subscribing at the discounted rate of Regular - $£ 70$ and Student $-£ 29$.

- Lunch and refreshments. Will be served at various catering points during scheduled breaks, including the banqueting suite and atrium. These are included as part of your delegate fee.

- Reception. The Orchard Hotel Reception is open 24 hours and is located in the hotel lobby

- Registration. Conference registration in the atrium and will be from 09.00 on Tuesday 28 November and 08:00 on Wednesday 29 November. On arrival for your first day it is important that all delegates register here to collect their delegate bags containing the Programme and Abstracts booklet.

- Taxis. Nottingham Cars are familiar with the venue, to book please call 01159700700.

- Travel directions and guidance. The conference is being held at the East Midlands Conference Centre, University Park, The University of Nottingham, NG7 2RJ. (Adjacent to the Orchard Hotel).

\section{- By Car:}

Leave the MI motorway at junction 25 and follow the A52 signposted to Nottingham. After approximately 4.4 miles turn right at the roundabout (Toby Carvery) onto the A6464 Woodside Road. Turn left at the next roundabout to enter the University of Nottingham's West Entrance.

The EMCC and Orchard Hotel is situated on

Beeston Road, which is the main loop road through the campus.

For satellite navigations systems please use postcode NG7 2QL.

\section{- By Rail:}

The nearest stations to the EMCC are Beeston Station and Nottingham. There are over 30 direct trains that travel between St Pancras and Nottingham on a daily basis.

\section{If you require a taxi please contact the EMCC reception team: 0844346 I2 I6.}

\section{- By Tram:}

The nearest Net Tram stops to the EMCC are:

- University Boulevard stop, I0-15 minute walk

- University of Nottingham stop - situated adjacent Lakeside Arts Centre - 20 minute walk through the University of Nottingham campus.

- Disclaimer. Full terms and conditions are available on the BASES Conference website. Please note that the hotel and organisers cannot be held liable for personal belongings or vehicles. Delegates are responsible for the safety of the valuables and property at all times.

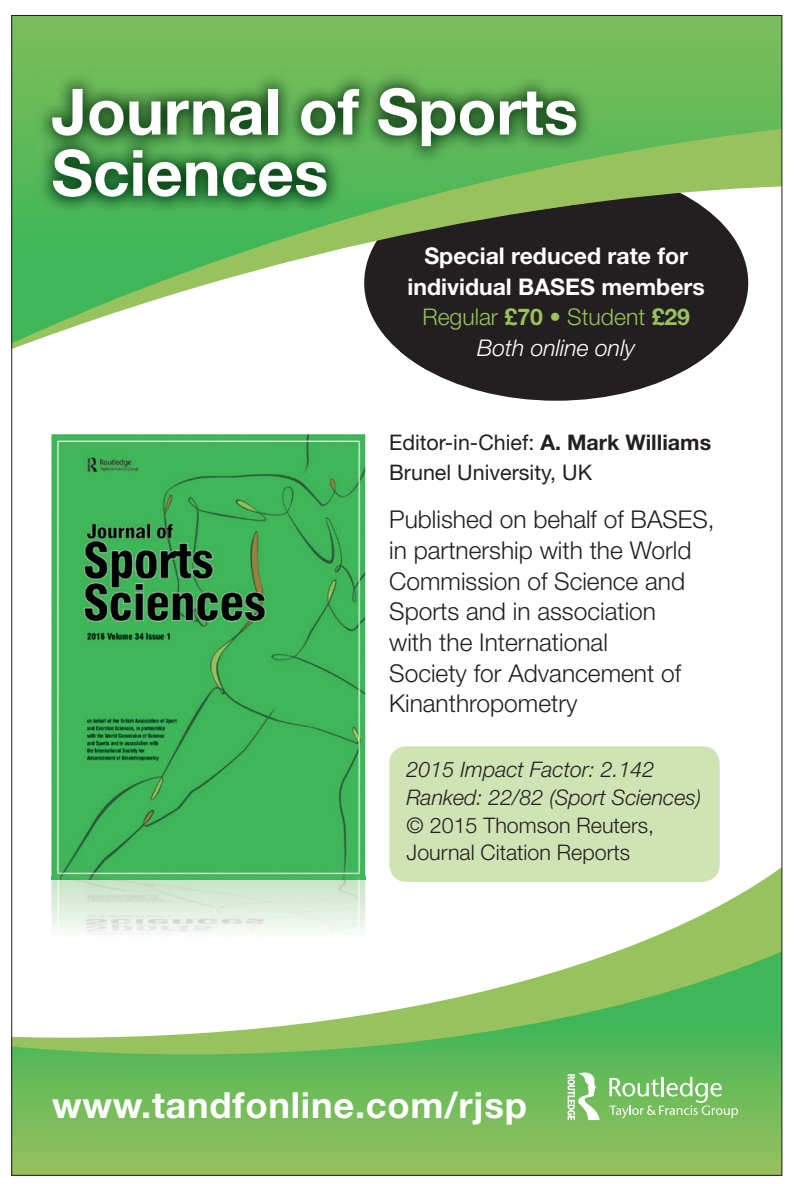




\section{BASES Fellowships}

The following individuals have been awarded BASES Fellowships this year.

The award recognises esteemed professional achievement, skills, knowledge and service to BASES and the sport and exercise science community.

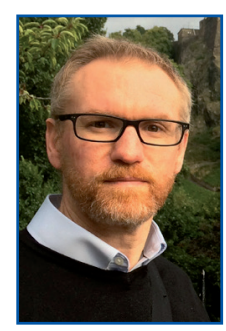

Dr Grant Abt

Grant is a Reader in Sport and Exercise Physiology at the University of Hull. Over the last 20 years he has had the privilege of teaching more than 2000 undergraduate and postgraduate students, including nine who now work for professional football clubs. He has been an undergraduate external examiner, external thesis examiner and reviewed papers for most peer-reviewed journals in our field. He has published almost 50 peer-reviewed journal articles with a citation count of $275 \mathrm{I}$ and a $\mathrm{H}$-index of 27 . Since $20 \mathrm{I} 2$ he has been an editor for the Journal of Sports Sciences, handling over 1000 manuscripts. He has been a sport science consultant with a range of professional clubs including Hull KR, West Ham FC, Liverpool FC, and GB Women's Hockey.

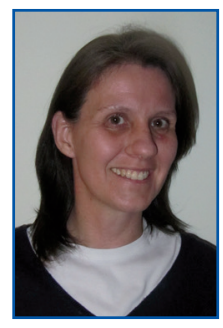

\section{Dr Lisa Board}

Lisa is a Senior Lecturer in Sport and Exercise Sciences at the University of Sunderland. She is an Accredited Sport and Exercise Scientist (since 1998) and a BASES Supervisor/Reviewer. She contributed to the BASES Position Stand on Curriculum-Based Work Placements and the BASES Position Stand on Graduate Internships and was previously a member of The Sport and Exercise Scientist Editorial Board. With a passion for teaching and learning she is a Senior Fellow of the Higher Education Academy. Her academic and research interests align to exercise for health and environmental physiology. She provides physiological support to athletes and those embarking on extreme charity challenges e.g., to high altitude. Current research is focused on the monitoring of cardiovascular disease risk factors in the ageing workforce.

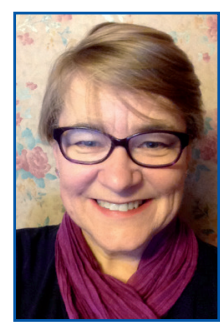

\section{Dr Val Cox}

Val studied cell biology and physiology of skeletal muscle in disease, before switching to applying these subjects in an exercise and health context. She served as BASES treasurer for 4 years and has managed the BASES conference awards for over 15 years. She has been based at Coventry University since 1999 and for 10 years headed the sports science team through various changes of Department/ Faculty, championing BASES membership for both staff and students. She ensured Coventry were one of the earliest courses to achieve endorsement, having been a member of the original committee that put BUES in place. Her most recent achievement was a major role in the design of the new Science and Health Building at Coventry, which includes greatly enhanced sport and exercise facilities.

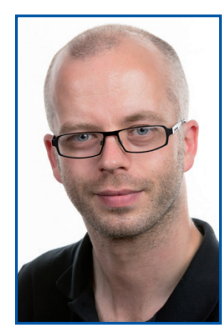

Dr Kevin Currell

Kevin has spent the last II years working in applied sport science practice at the EIS, firstly as a nutritionist, and more recently in leadership positions in sport science. He has multiple peerreviewed publications and a published book on Performance Nutrition. He has supported BASES through being on the editorial panel of The Sport and Exercise Scientist, serving on the BASES conference organising committee and delivering multiple presentations at BASES conferences and workshops.

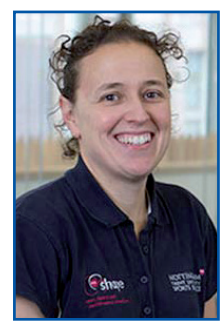

Dr Caroline Sunderland

Caroline is a Reader in Environmental and Sports Physiology at Nottingham Trent University, with a specific expertise in team sports activity in hot environments. She has been a BASES accredited Sport and Exercise Scientist for over 10 years for both Sports Science Support and Research and is a BASES network representative. She has worked with elite and recreational teams and individuals, of all ages and both sexes and has heat acclimated numerous Olympic teams and individuals prior to competing in hot environments. She has supervised probationary Sport and Exercise Scientists undertaking their accreditation and acted as a reviewer for the accreditation process. She has a passion for helping develop new and aspiring sports scientists.

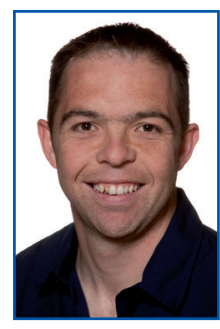

\section{Prof Craig Twist FBASES}

Craig is currently Professor of Applied Sports Physiology at the University of Chester. He has been a professional member of BASES for nearly 20 years and accredited since 2003. He has supported several candidates completing supervised experience, has acted as a reviewer and regularly facilitates the 'Understanding Your Client' workshop. In 20II, he was the conference organizer for the BASES Student Conference and has regularly attended student and annual conferences. He co-authored the BASES Position Stand on Curriculum-Based Work Placements and has contributed work to The Sport and Exercise Scientist. 


\section{Bases}

The British Association of

Sport and Exercise Sciences

\section{northumbria} UNIVERSITY NEWCASTLE

\section{BASES Student Conference 2018}

Contemporary issues in sport and exercise science - translating research into human performance

$12-13^{\text {th }}$ April 2018

Northumbria University,

Newcastle upon Tyne

For the conference programme and

information on travel \& accommodation please visit:

http://www.bases.org.uk/Student-Conference
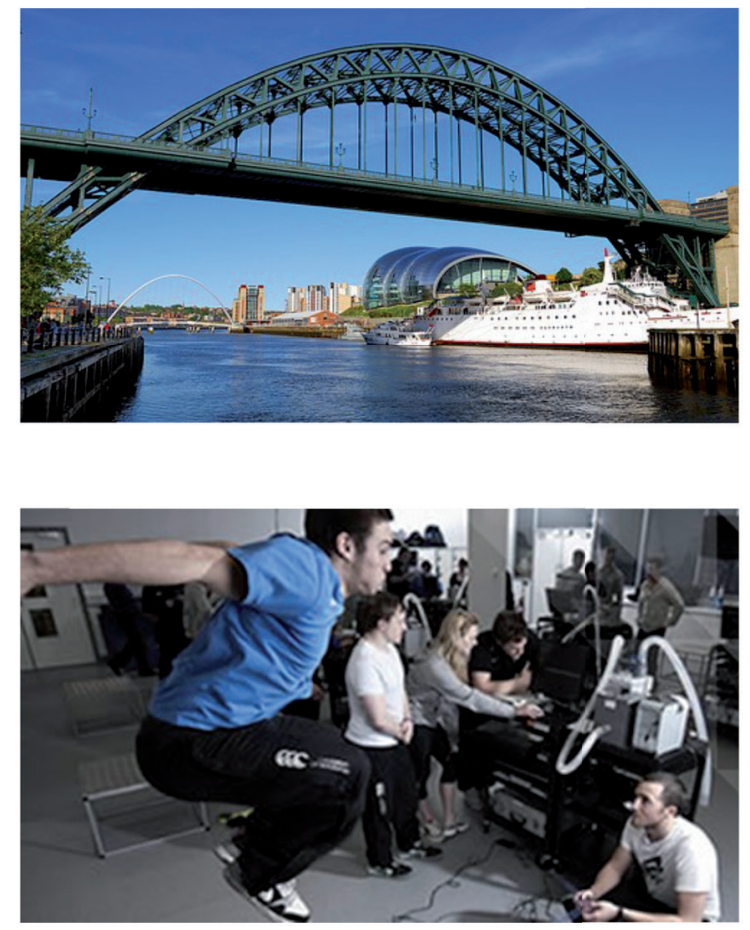

\section{Registration is now open! Abstract deadline - February $23^{\text {rd }}$}

\section{Contact}

Dr Stuart Goodall

stuart.goodall@northumbria.ac.uk

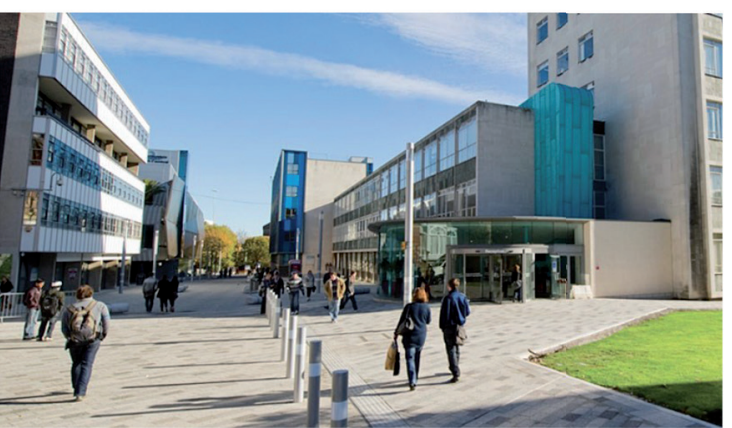




\section{Speaker}

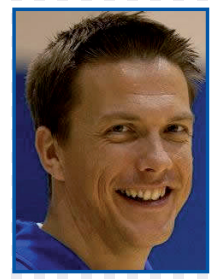

\section{Dr Angus Mugford} Toronto Blue Jays, Canada

Angus is the Director of High Performance for the Toronto Blue Jays, where he leads a team of specialists across Sport Medicine, Sport Science, Strength and Conditioning, and Mental Performance. Prior to this role, he served as the Director of the IMG Institute, which led mental performance and leadership consulting services for sport, corporate and military organisations.

\section{Developing a high performance culture: recommendations for research and practice}

\section{Invited keynote, DI.SI.}

\section{0:10-I I:10, Conference Theatre}

\section{Chair: Prof Chris Harwood FBASES}

This keynote will draw upon insights and experience of developing a high performance culture in North American professional sport, focusing on the challenges and opportunities that impact academics and practitioners in the field of applied sport science, particularly around interdisciplinary work in the context of professional environments.

Notes: 


\section{Speakers}

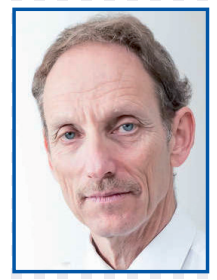

Prof Adrian Bauman University of Sydney, Australia

Adrian is Professor of Public Health and Director of the Prevention Research Collaboration at the University of Sydney in Australia. He leads the Physical Activity and Exercise theme in the Charles Perkins Centre, a research institute at Sydney University. He has a 20-year focus on physical activity and public health, with recent interests in the epidemiology of sedentary behaviour and health. $\mathrm{He}$ co-directs the WHO Collaborating Centre on Physical Activity, Nutrition and Obesity and is well published in the peerreviewed scientific literature.

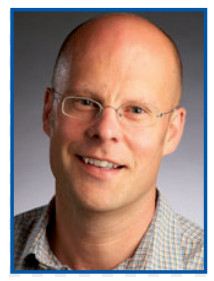

Prof Dylan Thompson University of Bath

Dylan is Professor of Human Physiology at the University of Bath. His research investigates the role of physical activity and exercise in the prevention of chronic disease. $\mathrm{He}$ integrates molecular and cellular laboratorybased approaches with free-living assessments outside the lab. He has published widely and he is regularly invited to give presentations at major conferences around the world. His research has been supported by major grants from the British Heart Foundation, BBSRC, MRC, Diabetes UK and the National Institute for Health Research. He serves as an advisor on funding agency panels and other bodies in the UK and overseas.

\section{Physical activity and sedentary behaviour: measurement, interpretation and implementation}

\section{Invited symposium, DI.S2.I}

\section{| |:30-|2:45, Suite |}

\section{Chair: Prof Mike Duncan FBASES}

In this session Prof Dylan Thompson will explain the multidimensional nature of physical activity and discuss some new techniques and approaches for physical activity monitoring. Prof Adrian Bauman will critically evaluate the evidence relating to sitting behaviour and health outcomes and will assess the challenges inherent in translating research into practice.

Notes: 


\section{Speakers}

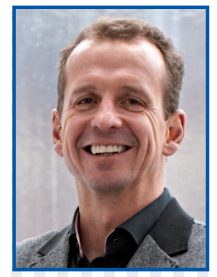

Prof Greg Whyte OBE, FBASES

Liverpool John Moore's University

In 2014 Greg was awarded an OBE for his services to sport, sport science and charity. He is an Olympian in modern pentathlon, and is a European and World Championship medallist. He is currently Professor of Applied Sport and Exercise Science at Liverpool John Moore's University and Director of Performance at the Centre for Health and Human Performance at 76 Harley Street, London. His former roles include Director of Research at the British Olympic Association and Director of Science \& Research for the English Institute of Sport.

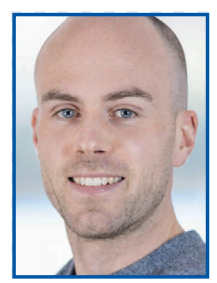

\section{Dr Jo Corbett} University of Portsmouth Jo is a Principal Lecturer and Associate Head in the Department of Sport and Exercise Science at the University of Portsmouth. He works in the Extreme

Environments Laboratory, which adopts an interdisciplinary research approach to understanding, evaluating and enhancing human comfort, performance, and survival in extreme environments. In his applied practice he has worked with athletes at all levels, including Olympic and World Champions.

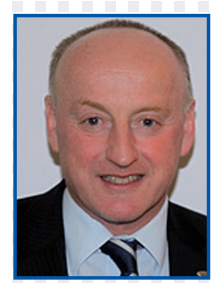

Prof Mike Tipton University of Portsmouth

Mike has spent over 35 years researching thermoregulation, environmental and occupational physiology and survival in the sea. He has published over 550 scientific papers, reports, chapters and the books The Essentials of Sea Survival and The Science of Beach Lifeguarding. He chaired UK Sport's Research Advisory Group and sits on the English Institute of Sports' Technical Advisory Group. $\mathrm{He}$ is a Fellow of the Royal Society of Medicine and provides advice to a range of universities, government departments, medical, search and rescue and media organisations. He is Editorin-Chief of The Physiological Society's journal, Experimental Physiology.

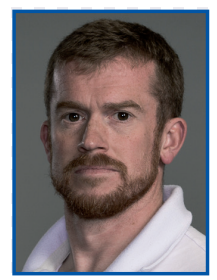

Mark Homer British Rowing

Mark joined British Rowing as a sports scientist in 2006 , delivering physiological services to elite rowers both in the UK and overseas, including a range of climates and altitude camps. He has supported the team at the Beijing, London and Rio Olympics, together with multiple World Championships. After Rio 2016 he was appointed Head of High Performance Science \& Medicine and now coordinates the research, strategy and delivery of Science and Medicine within the British team.

\section{Performance at the extremes: high, hot and cold}

\section{BASES expert statement highlight session}

Invited symposium, DI.S2.2.

\section{I:30- | 2:45, Suite 3}

\section{Chair: Prof Greg Whyte OBE, FBASES}

The ability to cope with the challenges of extreme environments is crucial for performance and health. Our understanding of the complex interaction of the human body in extreme environments has continued to evolve, leading to athletes pushing the limits of human performance. This session is a roundtable discussion of the BASES expert statement on performance and health in high, hot and cold environments.

Notes: 


\section{Speakers}

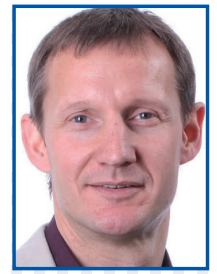

Prof Jens Kleinert

German Sport University, Cologne

Jens is Professor for Health and Sport Psychology and Head of the Institute of Psychology at the German Sport University, Cologne. He was formerly Professor for Sport and Health at the Institute of Sport Science, University of Würzburg, Germany. His main research interests are: interpersonal processes; motivation; wellbeing; and quality management in psychological counselling. He is the founder of the initiatives MentalTalent and MentallyPrepared, which focus on health promotion and the prevention of mental disease in competitive and elite sport.

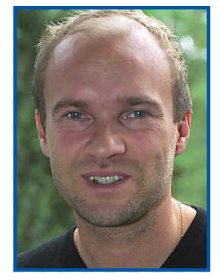

\section{Dr Göran Kenttä}

Swedish School of Sport and Health Sciences

Göran earned his doctorate in psychology at Stockholm University in 200l. His research has focused on elite-level athletes and the training process with a stress-recovery perspective, including clinical mental health issues of athletes and high-performance coaches. He currently holds a research position in Stockholm at the Swedish School of Sport and Health Sciences, where he has been a Director of the Coach Education Program. He currently holds a position at the Swedish Sport Federation as Head of Discipline in sport psychology.

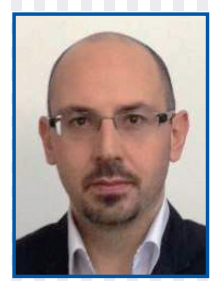

\section{Cédric Quignon-Fleuret} Institute of Sport, France

Cédric is a clinical sport psychologist working at the Institut National du Sport (Insep) in France. He is Head of the Sport Psychology Unit in the Insep medical department. He has worked with several Federations and athletes as a mental coach before joining Insep in 2005. His main tasks include clinical evaluations, athlete follow ups, collaboration with the medical department (doctors, dieticians, physiotherapists) and contributing to coaches' awareness of psychological dimensions in elite sport. He recently published his first book on sport psychology, Becoming champion: psychology to enhance performance.

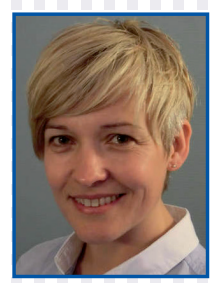

\section{Dr Kate Goodger} Chimp Management

Kate is a Director at Chimp Management and is a Chartered Sport and Exercise Psychologist and $\mathrm{HCPC}$ registered member. Her day to day work focuses on enhancing psychological functioning and performance in corporate and elite sport settings. She is proud to have been a psychologist with Team GB sports at six Olympic Games and is currently supporting GB Equestrian in the Tokyo cycle and Team GB Curling at the 2018 Winter Olympics. She completed her PhD in athlete burnout at Loughborough University in 2007.

\section{Mental health problems in elite athletes: systems, challenges and recommendations for practice}

Invited symposium, DI.S2.3.

\section{I |:30-12:45, Conference Theatre}

\section{Chair: Dr Karin Moesch}

This roundtable focuses on mental health problems in elite athletes. Participants from different countries will provide insight into how elite athletes with mental health problems can be supported, with a focus on prevention, detection and treatment of mental health problems in this specific population. Recommendations will be discussed to further enhance clinically oriented sport psychology services.

Notes: 


\section{Speakers}

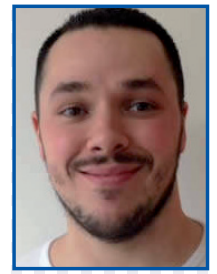

\section{Dr Benjamin Wall} University of Exeter

Benjamin obtained his BSc from the University of Birmingham and his $\mathrm{PhD}$ from the University of Nottingham Medical School where his thesis addressed the integration of fat and carbohydrate metabolism during exercise. Thereafter, he worked as a post-doctoral fellow at Maastricht University Medical Centre where he studied the nutritional regulation of muscle mass in health and disease. He is a Senior Lecturer in the School of Sport and Health Sciences at the University of Exeter where his research interests centre on how nutrition and physical (in)activity influence metabolic health and performance in a variety of populations.

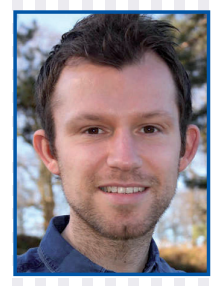

Dr Oliver Witard University of Stirling

Oliver is a senior lecturer at the University of Stirling, Scotland. His research interest is protein nutrition for maximising muscle adaptation to exercise training. His publications focus on establishing the optimal dose, source and timing of dietary protein for maximising gains in muscle mass, strength and performance. He has delivered several invited talks on protein nutrition at international/ national symposiums, including presentations at the Sport and Exercise Nutrition Register (SENr) meeting at Murrayfield Stadium and the International Sport and Exercise Nutrition Conference (ISENC) in Newcastle.

\section{The adaptive response of human skeletal muscle to physical (in)activity: considerations for nutritional optimisation}

Invited symposium, DI.S4.I.

16:05-I7:20, Suite I

\section{Chair: Prof Emma Stevenson}

Skeletal muscle tissue exhibits great plasticity depending on the mechanical strain imposed upon it. This session will discuss the physiological mechanisms underlying the adaptive response to increased, and decreased, physical activity; and address how the adaptive response to structured exercise training can be optimised via considerations relating to dietary protein. The session's conclusions will aim to provide up-to-date recommendations for dietary protein intake in endurance and resistance trained athletes. We will summarise practical considerations when transferring review intelligence and methodology within high performance sport environments.

Notes: 


\section{Speakers}

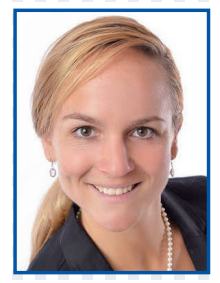

\section{Dr Ramona Ritzmann} University of Freiburg, Germany

Ramona is a lecturer

in Biomechanics at the

University of Freiburg.

Her major focus of work

comprises human movement

science with an expertise in the interconnection of neurophysiology and mechanics. This integration of transdisciplinary methodological approaches allows her to investigate the activity and plasticity of the central nervous system with respect to human movement control and motor learning. This research focus has led to numerous publications and invited presentations.

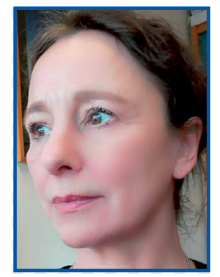

\section{Dr Claudine Lamoth} University of Groningen, Netherlands

Claudine is Associate

Professor at the Center of Human Movement Science of the University Medical Center Groningen. Her research

focuses on understanding the mechanisms underlying healthy ageing and age-related movement disorders, with an emphasis on gait and postural control. She uses concepts and tools from coordination dynamics, a theoretical approach that explicates variability as an inherent property of human movement, which provides an expedient window into the coordination of system components, as well as its stable and adaptive features. Central to her research is the quest for factors like coordination, cognition and energy cost, and their interaction in optimising and adapting postural and gait control.

\section{Biomechanical and neuromuscular challenges during ageing and disuse}

Invited symposium, DI.S4.2.

\section{6:05-17:20, Suite 4 \\ Chair: Dr Florentina Hettinga}

This session will provide an insight into adaptations in response to ageing, with a particular focus on biomechanical and neuromuscular changes. Data obtained about the mechanisms underlying degradations in response to inactivity during bed rest and immobilisation will be systematically reviewed and various countermeasures designed to reduce these effects will be discussed.

Notes: 


\section{Speakers}

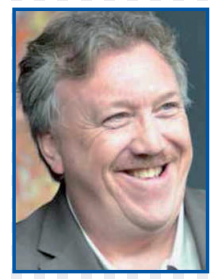

Prof Paul Wylleman

Vrije Universiteit Brussel, Belgium

Paul is a Professor at the

Vrije Universiteit Brussel, teaching sport psychology, high performance management and mental support for talented and elite athletes. His research and publications focus on athletic transitions, (dual) career management and sport psychology support services. He is Head of the Topsport and Study department and the research group Sport Psychology and Mental Support. He is past-President of the European Federation of Sport Psychology (FEPSAC) and high performance manager of the Performance Behaviour unit of the Netherlands Olympic Committee. Finally, he was the team psychologist to the Dutch Olympic team (TeamNL) at the 2016 Rio Olympic Games.

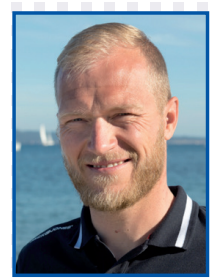

Dr Kristoffer Henriksen University of Southern Denmark

Kristoffer is Associate Professor at the Institute of Sport Science and Clinical Biomechanics at the University of Southern Denmark. His research in sport psychology takes a holistic approach and looks at social relations and their influence on athlete development and performance with an emphasis on successful sport environments. His employment includes a specialised function as a sport psychology practitioner in Team Denmark (national elite sport institution) with a focus on developing high performance cultures in national teams and mentally strong athletes and coaches. He was team sport psychologist for the Danish sailing team in the London and Rio Olympic Games.

\section{Challenges in the provision of psychology support for performance excellence during the Rio Olympic and Paralympic Games}

Invited symposium, DI.S4.3.

\section{6:05-17:20, Conference Theatre}

\section{Chair: Dr Rachel Arnold}

This session will highlight the contrasting challenges regarding the content, organisation and quality of support provided to Olympic and Paralympic athletes by team psychologists at Rio 20 16. Drawing from their experiences, critical insights will be offered on support provision; the organisation and effects of a solid performance culture; and on the competency profiles of practitioners working with elite athletes.

Notes: 


\section{Speakers}

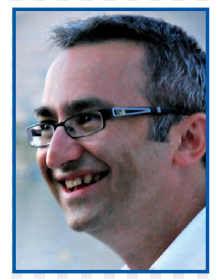

\section{Prof Antonis}

Hatzigeorgiadis

University of Thessaly, Greece

Antonis is an Associate

Professor of Performance

Psychology in Sport at the

Department of Physical

Education and Sport Science

at the University of Thessaly in Greece and

Treasurer of the European Federation of Sport

Psychology. He is heavily involved in exercise and health oriented research and recently completed a large project on the promotion of exercise for smoking and alcohol prevention and treatment.

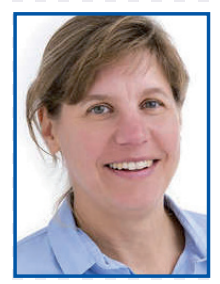

\section{Dr Anne-Marie Elbe} University of Copenhagen, Denmark

Anne-Marie is an Associate Professor of Sport and Exercise Psychology at the University of Copenhagen in Denmark and President of the European Federation of Sport Psychology. Due to her involvement in two large Danish research projects - the Copenhagen Women Study and the Copenhagen Center for Team Sport and Health - she is experienced in working in interdisciplinary research teams and has developed expertise on the health impact of physical activity interventions for different target groups.

\section{Interdisciplinary research on physical activity interventions targeting obesity and smoking cessation}

Invited symposium, DI.S4.4.

16:05-17:20, Suite 3

\section{Chair: Dr Anne-Marie Elbe}

This session will cover interdisciplinary research conducted on two physical activity interventions. The first targets smoking cessation and the collection of psychological and biochemical data. The second investigates the effects of an intense lifestyle intervention for individuals with obesity, combining a physiological, psychological and sociological research perspective. Advantages and challenges of interdisciplinary physical activity research will be outlined.

Notes: 


\section{Speaker}

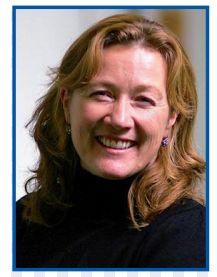

Prof Cathy Craig Queen's University, Belfast, Northern Ireland

Cathy is Professor of

Perception and Action

Psychology and Dean for

Postgraduates at Queen's

University, Belfast. She

conceived, designed and developed the state of the art Movement Innovation Lab that houses multi-sensory immersive technologies and motion capture systems that allow the study of how people make decisions about action. She has applied the theoretical principles of her work to the design of immersive systems that help improve movement performance in sport and health. Her work has been recognised through the award of a prestigious ERC grant and nominations for innovation in health awards.

\section{Virtual reality technology: what use can it have in sport?}

Invited keynote, D2.S2.

09:15-10:10, Conference Theatre

\section{Chair: Prof Markus Raab}

This keynote will show how immersive, interactive virtual reality technology is used to study decision-making in sport. The versatility of this technology means it can be easily applied to many sports and allows for the accurate recording of players' movement responses. By manipulating the visual information presented to players in the virtual world and subsequently measuring when and how they respond, new insights into players' decisionmaking abilities can be obtained.

Notes: 


\section{Speakers}

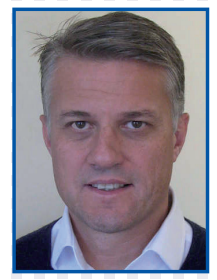

Prof Steve Harridge Kings College London

Stephen obtained his PhD from the University of Birmingham and undertook post-doctoral research at the Karolinska Institute, Sweden and Copenhagen Muscle

Research Centre, Denmark. He has worked in the University Department of Geriatric Medicine at the Royal Free Hospital School of Medicine and in the Department of Physiology at University College London, where he was a Wellcome Trust Research Fellow. Since 2005 he has been Professor of Human and Applied Physiology at King's College London and is Director of the Centre of Human \& Aerospace Physiological Sciences within the Faculty of Life Sciences and Medicine. He is Editor in Chief of the Scandinavian Journal of Medicine and Science in Sports. Using cell biology through to exercise physiology approaches, his research focuses on increasing understanding of human skeletal muscle function and plasticity, particularly in regard to ageing.

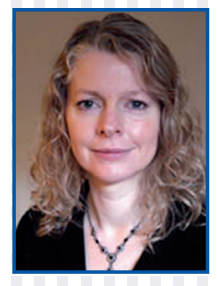

\section{Dr Katherine Brooke-} Wavell Loughbrorough University

Katherine is a Senior Lecturer in the School of Sport and Exercise Sciences at Loughborough University, based in the National Centre for Sport and Exercise Medicine East Midlands Hub. Her research focuses on the effects of exercise on bone health. She has led a number of exercise intervention trials, ranging from studies of bone health and stress fracture in elite athletes, through studies of high impact exercise and brisk walking in middle aged and older adults, to research on the effectiveness of fall prevention exercise and whole body vibration training in older people. Outcomes studied include bone mineral density and structural parameters, bone turnover and fall risk factors as well as body composition, physical function and cardiovascular risk factors.

\section{Exercise in older adults}

Invited symposium, D2.S3.I.

10:15-1 I:10, Suite I

\section{Chair: Dr Miranda Armstrong}

In this session Prof Steve Harridge will examine the decline in physiological systems that occur with ageing and discuss studies in master's athletes to assess the extent to which declines in functional capacity are due to ageing per se versus physical inactivity. Dr Katherine Brooke-Wavell will then share her knowledge on the potential for exercise to increase bone strength and reduce the risk of falls and osteoporotic fractures in older adults. This will include an examination of the different types and intensities of exercise for increasing bone density.

Notes: 


\section{Speakers}

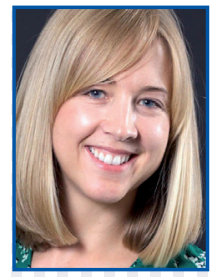

Prof Susan Backhouse FBASES

Leeds Beckett University

Susan is Director of Research at the Institute for Sport, Physical Activity and Leisure and Professor of Psychology and Behavioural Nutrition

at Leeds Beckett University. She was an invited member of the EU Expert Group on Doping in Sport and was involved in the drafting of EU recommendations for the prevention of doping in recreational sport. She and her research team at Leeds Beckett have attracted funding from the World Anti-Doping Agency, International Olympic Committee, European Commission and National Governing Bodies of Sport. She convened the BASES Clean Sport interest group and she is a member of the World Anti-Doping Agency Social Science Committee.

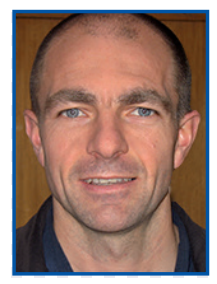

\section{Dr lan Boardley} University of Birmingham

lan is a senior lecturer in Sport Psychology at the University of Birmingham's School of Sport, Exercise and Rehabilitation Sciences. He is internationally recognised for his expertise on the psychosocial processes governing moral behaviour in sport, exercise, and dance. This work includes moral behaviours that occur on the field of play (e.g. pro-social and anti-social behaviour), as well as those that occur off it (use of performance and cognitive enhancing drugs). lan's research has been supported by funding from the World Anti-Doping Agency, the International Olympic Committee and the Economic and Social Research Council.

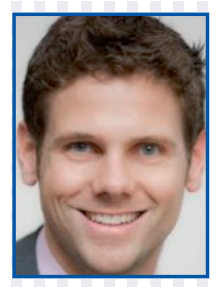

\section{Mike Stow} English Institute of Sport Mike has been in High Performance Sport since 2006, undertaking roles with UK Sport, UK Anti-Doping and the English Institute of Sport. After Graduating in

Physiology \& Pharmacology from the University of Leeds, Mike went on to establish and lead the Science and Medicine team at UK Anti-Doping prior to moving over to an operational role for the EIS in Manchester. As part of his current role Mike leads on the EIS Clean Sport strategy.

\section{Inadvertent doping in sport: protecting the athlete, the practitioner and the profession}

\section{BASES expert statement highlight session}

Invited symposium, D2.S3.2.

\section{0:15-I I: I0, Suite 3}

\section{Chair: Prof Susan Backhouse FBASES}

This session will consider the controversial issue of doping in sport, and in particular, the unintentional use of prohibited substances or methods. This session will use collaborative technology to activate the BASES community and gauge their stance on the BASES expert statement on inadvertent doping in sport.

Notes: 


\section{Speakers}

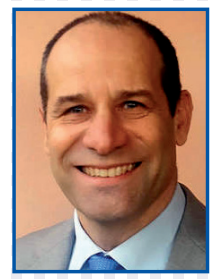

\section{Dr Maurizio Bertollo} University of Chieti-Pescara, Italy

Maurizio is Associate Professo of Motor Behaviour and Sport Psychology at "G. d'Annunzio" University of Chieti-Pescara in Italy, where he is Associate Director of the Behavioural Imaging and Neural Dynamics (BIND) Centre. His research focuses on the processes and mechanisms underlying the development, maintenance and improvement of human motor behaviour and performance. Current research interests include bio-psychophysiological state underpinning performance, individual zones of optimal functioning, stress recovery-balance in sport, psychophysiological monitoring and intervention in sport and the psychophysiological correlates of athletic performance.

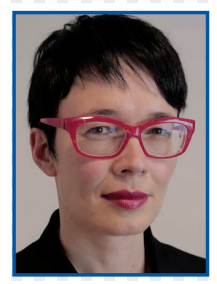

Prof Lorenza Colzato Leiden University, Netherlands

Lorenza is Professor by Special Appointment of Cognitive Enhancement at Ruhr University Bochum in Germany. She is also Senior Research Fellow at the Institute for Sports and Sport Science at the University of Kassel and VIDI Research Fellow at the Institute of Psychological Research at Leiden University in the Netherlands. Her research activity focuses on cognitive enhancement; the use of any means (e.g. brain stimulation, meditation, physical exercise, lifestyle or food supplements) aimed at enhancing performance in healthy individuals. Current research interests include how brain stimulation and neural entrainment, relying on non-invasive techniques, might enhance brain activity in healthy athletes to improve their physical performance.

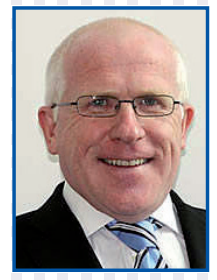

\section{Prof Christopher Ring} University of Birmingham

Christopher is Professor of Psychology at the University of Birmingham. He has published over 150 articles and received grant funding from research councils,

charities and industry. He has served as Associate Editor of Biological Psychology for over 10 years. He is known for his approach to science that crosses traditional disciplinary boundaries and has conducted programmatic research concerning cardioception, stress and immunity and hypertensive hypoalgesia. His psychophysiological research on learning and performance spans over 30 years and these days he is focused on identifying the mechanisms that underpin optimal performance.

\section{Sport psychophysiology}

Invited symposium, D2.S3.3.

10:15-1 I:10, Conference Theatre

\section{Chair: Dr Maurizio Bertollo}

This session will provide an overview of the psychophysiological features of optimal and suboptimal performance in sport. Presenters will discuss the neural markers underlying performance experience and the effect of non-invasive brain stimulation on performance. The conclusion of this synthesis is applicable within performance enhancement and mental training programmes, providing a comprehensive perspective on psychophysiological intervention in sport.

Notes: 


\section{Speakers}

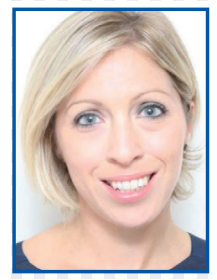

Prof Emma Stevenson Newcastle University

Emma is Professor of Sport and Exercise Science in the Institute of Cellular Medicine in the Faculty of Medical

Sciences at Newcastle University. She is also a PI in the Human Nutrition Research Centre at Newcastle University. Her research focuses on the effects of exercise and nutritional interventions on appetite regulation and postprandial metabolism. Her recent work has focused on dairy and dairy proteins and their role in health, postprandial metabolism and the regulation of food intake across the lifecourse.

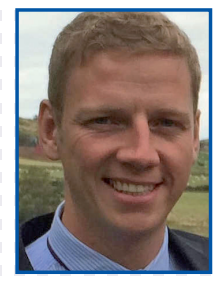

\section{Dr Lewis James}

\section{Loughborough University}

Lewis is a Senior Lecturer in Nutrition within the School of Sport, Exercise and Health Sciences at Loughborough University, and is Programme Director for the MSc Sport and Exercise Nutrition. Broadly, his research examines the singular and combined effects of exercise and nutrition on human metabolism and behaviour, with specific foci on the role of exercise and nutrition in energy balance regulation; and the role of nutrition in optimising exercise performance. Current research projects include those examining the impact of acute energy restriction/exercise on human appetite, eating behaviour and performance.

\section{Nutrition, exercise and appetite: hungry for more?}

Invited symposium, D2.S5.I.

|3:45- |4:45, Suite 3

\section{Chair: Dr Mike Price FBASES}

This session will focus on the impact of alterations in energy balance through energy restriction and exercise on appetite and energy balance regulation. There will be particular focus on novel dietary strategies to attenuate energy balance, including intermittent severe energy restriction and time restricted feeding.

Notes: 


\section{Speakers}

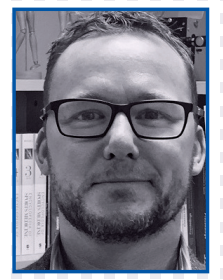

Prof Gareth Irwin CardiffMetropolitan University

Gareth is Professor of Sports Biomechanics at Cardiff Metropolitan University and is Fellow and President of the International Society of

Biomechanics in Sport. He is a Visiting Professor at the University of Ostrava, Czech Republic and a Research Associate at the Auckland University of Technology, New Zealand. His research philosophy is driven by a desire to understand sporting performance using ecologically valid scientific methods to address meaningful questions that inform researchers, coaches, and clinicians. Two themes reflect this philosophy: The coaching biomechanics interface and Sports medicine and injury. He has over 130 peerreviewed publications including books, papers and conference abstracts.

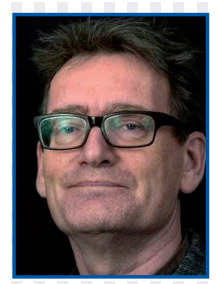

Prof Bert Otten University of Groningen Netherlands

Egbert Otten studied biophysics at Leiden University and graduated in 1978 after which he wrote his thesis on movement contro and evolution of fish, which received a national award. Between 1982 and 1984 he worked at the medical faculty of the Free University in Amsterdam. Since 1984 he has worked at the University of Groningen, during which he was guest scientist at Harvard University, University of Calgary and NIH in Bethesda (USA). Since 2005, he has been a Professor of Neuromechanics, in which his main themes are movement control, biomechanics and motor learning in humans.

\section{Analysing, modelling and improving Olympic performance}

Invited symposium, D2.S5.2.

I 3:45- | 4:45, Suite 4

\section{Chair: Adam Hawkey}

Many aspects of Olympic performance can be analysed with motion capture and simulated with custom models. Drawing on current research examples from amputee sprinting, gymnastics, track cycling and speed skating, this session will demonstrate how ecologically valid research in biomechanics, aerodynamics and motor control can enhance the performance of Olympic athletes.

Notes: 


\section{Speakers}

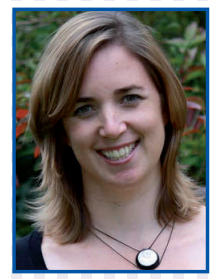

Dr Jennifer Cumming University of Birmingham Jennifer is a senior lecturer in Sport and Exercise Psychology at the University of Birmingham, a Chartered Psychologist and Associate Fellow of the British

Psychological Society, and a Fellow of the Higher Education Academy (HEA). She has published over 80 peer-reviewed papers and is the current co-editor of Imagination, Cognition and Personality. She is the Primary Investigator of the Mental Skills Training for Life programme (\#MST4Life) for developing resilience, selfworth and mental well-being in homeless young people with complex needs.

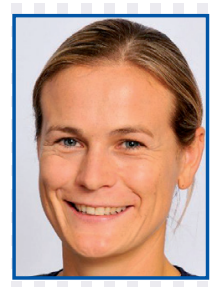

\section{Dr Camilla Knight} Swansea University

Camilla is a Senior Lecturer in Sport and Exercise Psychology at Swansea University, a BASES accredited sport and exercise scientist, and the youth sport lead for the Welsh Institute of Performance Science. She has published over 30 peerreviewed papers pertaining to youth sport, is co-author of Parenting in Sport: From Research to Practice and co-editor of the forthcoming text on Sport Psychology for Young Athletes. She is currently involved in a number of projects relating to parental involvement in youth sport, as well as delivering sport psychology programmes to young athletes.

\section{Uncovering the process of a strength-based approach to mental skills training with young people}

Invited symposium, D2.S5.4.

\section{3:45-14:45, Conference Theatre}

\section{Chair: Prof Zoe Knowles FBASES}

This session will explore the delivery and evaluation of strength-based approaches to mental skills training in elite sport and hard to reach populations. Strategies and activities will be shared which have facilitated the application of mental skills training across various settings, as well as insights into the successes and challenges encountered in delivering strength-based mental skills training programmes.

Notes: 


\section{Speakers}

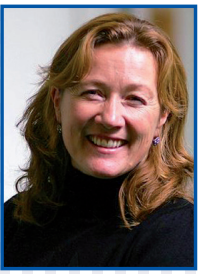

Prof Cathy Craig Queen's University, Belfast Northern Ireland

Cathy is Professor of

Perception and Action Psychology and Dean for

Postgraduates at Queen's University, Belfast. She conceived, designed and developed the state of the art Movement Innovation Lab that houses multi-sensory immersive technologies and motion capture systems that allow the study of how people make decisions about action. She has applied the theoretical principles of her work to the design of immersive systems that help improve movement performance in sport and health. Her work has been recognised through the award of a prestigious ERC grant and nominations for innovation in health awards.

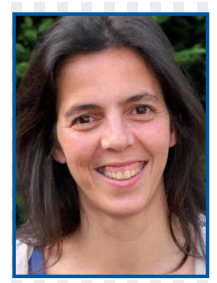

Prof Rita Cordovil University of Lisbon, Portugal

Rita is Assistant Professor at the Faculty of Human Kinetics, University of Lisbon, Portugal. She teaches Motor Development and her research uses direct perception, mostly applied to child safety issues. She studies the behavior of infants and children in different 'risk environments', such as water environments or high places. Her research interests include understanding how different children perceive affordances in risk settings and how adults perceive those affordances for children

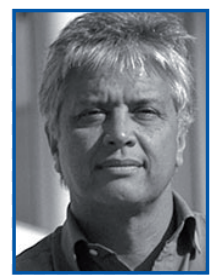

\section{Prof Keith Davids} Sheffield Hallam University

Keith is Professor of Motor Learning at the Centre for Sports Engineering Research, Sheffield Hallam University. He has held positions in the UK, New Zealand, Australia and Finland. His research programme in ecological dynamics investigates constraints on emergent coordination tendencies in athletes and sports teams. He has conducted research on elite sport programmes at the New Zealand South Island Academy, Queensland Academy of Sport, the Australian Institute of Sport, Diving Australia, Cricket Australia and the English Institute of Sport.

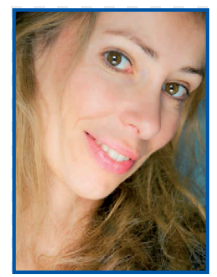

\section{Dr Rita de Olivera} London South Bank University Rita is a senior lecturer in Sport and Exercise Science at London South Bank University with a research focus on the reciprocity between perception and action. Her research uses a direct perception approach applied to various contexts from top-level performance to activities of daily living across the lifespan. Specifically, she has ongoing projects on perceptual-motor recalibration, multi-tasking and decision-making.

\section{Direct perception in applied contexts}

Invited symposium, D2.S5.4.

\section{3:45-14:45, Suite I \\ Chair: Dr Rita de Oliveira}

The direct perception approach posits that perception is unmediated by cognition, with consequences to how we organise both research and practice. Talks in this symposium will explain how key concepts from this approach can be used to guide research methodology and practical applications in exercise and sport.

Notes: 


\section{Speakers}

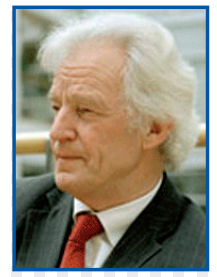

Prof Sir Muir Gray CBE Nuffield Department of Surgical Sciences, University of Oxford

Muir entered the public health service by joining the City of Oxford Health Department in 1972. The first phase of his professional career focused on disease prevention and health in old age, followed by the development of the NHS screening programmes, as well as services aimed at bringing knowledge to patients and professionals, including NHS Choices. He has been instrumental in setting up the Centre for Evidence Based Medicine and the Cochrane Collaboration in Oxford. During this period he was appointed as the Chief Knowledge Officer of the NHS in England. He is a Visiting Professor in Knowledge Management at the Department of Surgery and a Visiting Professor of Value Based Healthcare at the Nuffield Department of Primary Care Health Sciences. He has returned to his first mission to prevent disease and promote health in old age with a series of books: Sod60!, Sod70!, Sodlt! Eat Well and the Mid-life Health Handbook.

\section{Exercise versus pharma: what is the future of healthcare?}

Invited keynote, D2.S6.

\section{| 4:55-I5:45, Conference Theatre \\ Chair: Prof Greg Whyte OBE, FBASES}

Prof Greg Whyte OBE, FBASES will host a discussion with Prof Sir Muir Gray CBE on the role of exercise in health and primary care. Expect stimulating debate, including audience participation, on the role of exercise in the prevention, treatment and management of disease; and thoughts on how the health service can move forward.

Notes: 


\section{The British Association of Sport and Exercise Sciences Expert Statements}

\section{D1.S2.2. The BASES expert statement on interventions for improving performance in the heat}

JO CORBETT ${ }^{1 *}$, NEIL MAXWELL ${ }^{2}$, CAROLINE SUNDERLAND ${ }^{3}$, NEIL WALSH ${ }^{4} \&$ OLIVER GIBSON ${ }^{5}$

${ }^{1}$ University of Portsmouth, ${ }^{2}$ University of Brighton

${ }^{3}$ Nottingham Trent University, ${ }^{4}$ Bangor University,

${ }^{5}$ Brunel University London

*Corresponding author: jo.corbett@port.ac.uk

The detrimental influence of heat on prolonged exercise is well established, yet many individuals are required to exercise in the heat, resulting in a need for practical, evidencebased interventions for able-bodied individuals performing continuous or intermittent exercise (>30 min) in hot environments. Acclimation (laboratory) or acclimatisation (natural environment) to heat (HA) reduces initial deep-body temperature and increases heat dissipation rates. HA is achieved by repeatedly elevating deep-body and skin temperature and eliciting pronounced sweating, typically through exercise in a hot environment, although hot-baths $\left(\leq 40^{\circ} \mathrm{C}\right)$ of $15-$ 40 min following training are also effective (Zurawlew et al., 2016, Scandinavian Journal of Medicine and Science in Sports, $26,745-754)$. The use of $60-90 \mathrm{~min}$ daily exposures are common with active approaches and $\sim 10$ days is superior to $\sim 5$ days HA (Neal et al., 2016, Frontiers in Physiology, 7, 564). Short programmes still elicit significant adaptations although adaptation may be slower in females (Mee et al., 2015, Scandinavian Journal of Medicine and Science in Sports [Suppl 1], 250-258). Maintaining thermal-strain over the HA period, by increasing work-rate or environmental stress as the individual adapts, should be superior to exercise at a fixed work-rate and environmental stress, where the stimulus diminishes as the individual adapts. A target deep-body temperature of $\sim 38.5^{\circ} \mathrm{C}$ is common; higher temperatures appear unnecessary and increase heat-illness risk. HA is well-retained for 7 days and any benefits lost within a month of HA are reacquired with 2-4 further exposures. Individuals should commence exercise in the heat in a euhydrated state and prevent excessive dehydration ( $>2 \%$ body weight loss from water deficit), which impairs heat-loss. However, excessive drinking can cause hyponatremia, which is life-threatening, and individuals should drink to thirst unless they have a prescribed fluid-strategy. Pre- or per-(during) exercise cooling techniques can be effective (Tyler et al., 2015, British Journal of Sports Medicine, 49, 7-13) and include: (1) external cooling, e.g. cool water immersion, cooling garments; (2) internal cooling, e.g. ingesting ice-slurry beverage; and (3) mixed-methods (multiple internal and/or external techniques). Water immersion is often used, with temperatures of $12-18^{\circ} \mathrm{C}$, durations of 20-30 $\mathrm{min}$, and deep-body temperature monitoring for safety. Typically, $7.5 \mathrm{~g} \cdot \mathrm{kg}^{-1}$ body mass of cold-liquids (carbohydrate/electrolytes) or ice-slurry are ingested in the
20-30 min before exercise. Theoretically, mixed-methods should generate the greatest heat-sink. Phase change garments, e.g. 'ice vests', may inhibit some evaporation and may be more appropriate when evaporative heat-loss is restricted, e.g. protective clothing. The efficacy of ventilated vests is reduced with hotter, more humid air. Some interventions induce cool sensations (e.g. L-menthol, icepacks on the neck) with minimal influence on thermal state. We advise caution with perceptual manipulations, which may result in dangerously high body temperatures whilst masking signs and symptoms of heat-illness. The optimal intervention(s) must be determined on a case-by-case basis taking into account mechanisms, efficacy, practical and logistical constraints and safety issues.

\section{D2.S3.2. The BASES expert statement on} inadvertent doping in sport

\section{SUSAN H. BACKHOUSE ${ }^{1 *}$, IAN BOARDLEY ${ }^{2}$, NEIL CHESTER ${ }^{3}$, KEVIN CURRELL $^{4}$, AMANDA HUDSON ${ }^{5}$ KATE MILLS ${ }^{6} \&$ MIKE STOW $^{4}$}

\author{
${ }^{1}$ Leeds Beckett University, ${ }^{2}$ University of Birmingham, \\ ${ }^{3}$ Liverpool John Moores University, ${ }^{4}$ English Institute of \\ Sport, ${ }^{5}$ United Kingdom Anti-Doping, ${ }^{6}$ British Association \\ of Sport \& Exercise Sciences \\ *Corresponding author: \\ s.backhouse@leedsbeckett.ac.uk \\ @susanbackhouse
}

Doping is a threat to the health and well-being of athletes and the integrity of sport. Although attention often focuses on athletes who dope intentionally, doping can also occur inadvertently. Inadvertent doping is defined as the unwitting ingestion of substances prohibited by the World Anti-Doping Agency. Due to the principles of strict liability inherent in the World Anti-Doping Code, athletes are liable for the substances found in their body regardless of how they are ingested. Thus, athletes who do not appropriately guard against inadvertent doping are at risk of incurring anti-doping rule violations (ADRV). As the consequences of such ADRVs can be significant (i.e. 2 years of sport ineligibility), it is critical athletes and the personnel that support them are fully informed on the potential causes of inadvertent doping and how to protect athletes against them. The three most common causes of inadvertent doping are through use of: (1) medication; (2) contaminated supplements; and (3) contaminated food. The first of these - medication use - can result in inadvertent doping if a medication contains a prohibited ingredient and an athlete uses it either without being aware the ingredient was present, or without knowing the ingredient was prohibited. Additionally, certain drugs (e.g. pseudoephedrine) are only prohibited above a threshold level. If athletes mismanage use of such medications they may unknowingly surpass the threshold level. The 
second common cause - use of contaminated supplements - has accounted for numerous ADRVs. The risk posed by using dietary supplements that have not been tested for the presence of prohibited substances is highlighted by studies that evidence presence of prohibited substances (e.g. anabolic steroids; Geyer et al., 2004) in over-the-counter supplements. The final common cause - eating contaminated food - occurs through consumption of food products containing prohibited substances (e.g. meat containing cattle growth promoters [e.g. clenbuterol]). Use of such promoters is illegal in many world regions (e.g. European Union) and athletes living in such regions are at risk when traveling to regions where their use is legal (e.g. USA). Additional risks for inadvertent doping include sabotage, shared smoking and direct intimate contact. Sport and exercise scientists can help diminish the risk by equipping themselves - and the athletes they support with the capability, opportunity and motivation to mitigate against it. More specifically: (1) capability, by raising awareness of the risks to inadvertent doping; (2) opportunity, by creating opportunities for qualified professionals to provide necessary support; and (3) motivation, by reinforcing and promoting a sporting culture that prioritises athlete welfare over winning. 


\section{Day 1. Free Communications - Physical Activity for Health}

\author{
D1.S3.1(1). Cardiometabolic responses to \\ interrupting prolonged sitting with high-intensity \\ physical activity versus a continuous moderate- \\ intensity physical activity bout
}

\section{DANIEL BAILEY*, BENJAMIN MAYLOR, CHARLIE ORTON \& JULIA ZAKRZEWSKI-FRUER}

\section{University of Bedfordshire \\ *Corresponding author: daniel.bailey@beds.ac.uk @DrDanielBailey}

Sedentary behaviour and physical inactivity independently increase the risk of cardiometabolic disease (Healy et al., 2008, Diabetes Care, 31, 369-371). Interrupting prolonged sitting with moderate-intensity physical activity induces beneficial cardiometabolic responses that may be similar or more pronounced compared with a single energy and intensitymatched physical activity bout. However, the cardiometabolic response to interrupting prolonged sitting with high-intensity physical activity compared with continuous moderate-intensity physical activity is not known. Therefore, this study investigated the postprandial cardiometabolic response to interrupting prolonged sitting with high-intensity physical activity versus an energy-matched continuous moderateintensity physical activity bout. With institutional ethical approval, fourteen sedentary adults completed three, $8 \mathrm{~h}$ experimental conditions in a counter-balanced randomised order: (1) uninterrupted sitting (SIT), (2) uninterrupted sitting after a continuous 30 min moderate-intensity physical activity bout at $60 \%$ maximum oxygen uptake reserve (V O2R) (CONTSIT), and (3) sitting interrupted hourly with $2 \mathrm{~min} 32 \mathrm{~s}$ highintensity physical activity ( $85 \%$ V O2R) (SIT-ACT). The CONT-SIT and SIT-ACT conditions were matched for activity energy expenditure and all physical activity was on a motorised treadmill. Standardised meals were consumed at $0 \mathrm{~h}$ and $4 \mathrm{~h}$ providing $15 \%$ and $30 \%$, respectively, of estimated daily energy requirements for each participant. Venous blood samples were collected hourly. Incremental area under the curve (iAUC) was calculated for glucose, insulin, triglycerides, and high-density lipoprotein cholesterol (HDL) for each $8 \mathrm{~h}$ condition $\left(\mathrm{mmol} / \mathrm{L} \cdot 8 \mathrm{~h}^{-1}\right)$. Linear mixed models compared dependent variables between the conditions. Data are presented as mean (95\% confidence intervals). Activity energy expenditure did not differ between CONT-SIT $(661 \mathrm{~kJ}[476,828])$ and SITACT $(732 \mathrm{~kJ}[539,891] ; P=0.236)$. Postprandial glucose and insulin iAUC did not differ between conditions $(P \geq 0.606)$. There was a main effect of condition $(P=0.038)$ for triglyceride iAUC with concentrations being lower in SIT-ACT than SIT $(-0.88[-2.73,0.97]$ and $1.36[-0.46,3.17]$, respectively; $P=$ $0.035)$. There was also a main effect of condition $(P=0.034)$ for HDL iAUC with concentrations being higher in SIT-ACT than $\mathrm{SIT}(0.86[0.08,1.65]$ and $-0.13[-0.91,0.66$, respectively; $P=0.037$ ). No differences were observed between CONT-SIT and any other condition for triglyceride or HDL iAUC $(P \geq$ $0.211)$. Interrupting prolonged sitting with short, hourly highintensity physical activity resulted in beneficial lipid responses compared with uninterrupted sitting. A continuous bout of moderate-intensity physical activity did not attenuate the adverse cardiometabolic effects of uninterrupted sitting. These findings suggest that interrupting prolonged sitting with high-intensity physical activity may be an appropriate strategy to reduce cardiometabolic disease risk.

D1.S3.1(2). The utility of the Supine to Stand test as a measure of functional motor competence in children aged 5-9 years old

\section{MICHAEL DUNCAN ${ }^{1 *}$, CHELSEY LAWSON ${ }^{1}$, LEANNE WALKER ${ }^{1}$, DAVID STODDEN ${ }^{2} \&$ EMMA EYRE $^{1}$}

\author{
${ }^{1}$ Coventry University, ${ }^{2}$ University of South Carolina \\ *Corresponding author: \\ michael.duncan@coventry.ac.uk \\ @mikedunky
}

The supine to stand (STS) test offers potential as a global measure of functional motor competence and evidence suggests it offers a time efficient tool that has clinical and practical relevance for lifespan movement assessment $(\mathrm{Ng}$ et al., 2013, The Journal of Pediatrics, 162, 552-556; Klima et al., 2016, Journal of Aging and Physical Activity, 24, 207-213). Competence in moving from supine to standing may also be a precursor to the development of other fundamental movement skills (FMS). Few studies have examined this measure in children and none have examined whether FMS differs between children with better or poorer STS performance. This study sought to examine this issue. Following institutional ethics approval and informed parental consent, 91 children (47 males, 44 females) aged 5-9 years (Mean age \pm $\mathrm{SD}=6.8 \pm 1.2$ years) took part in this study. Height $(\mathrm{m})$ and body mass $(\mathrm{Kg})$ were assessed using a Seca Stadiometre and scales (SECA Instruments, Germany) from which body mass index $\left(B M I, \mathrm{~kg} / \mathrm{m}^{2}\right)$ was determined. FMS was determined using video analysis of four skills (run, jump, catch, throw) using the Test of Gross Motor Development-2 (Ulrich, 2001, Test of Gross Motor Development, Texas: PRO-ED). Total FMS was derived from the summed components of the skills. STS performance was assessed using procedures previously documented ( $\mathrm{Ng}$ et al., 2013). Following familiarisation, participants assumed a supine position on a padded mat on the floor. They were asked to stand up as quickly as possible following a 'go' command. Two trials were video recorded in the sagittal plane with the fastest time to complete the STS movement taken as a measure of functional motor performance. Tertiles were created for STS time scores, reflecting low, medium and high STS competence. Results from a 2 (Sex) $X 3$ (STS competency) ANCOVAs, controlling for age and BMI, indicated that boys had significantly better FMS than girls $(P=$ $0.006, \mathrm{P \eta}^{2}=.102$ ) and that FMS differed depending on STS tertile $\left(P=0.04, \mathrm{P \eta}^{2}=.087\right)$. FMS was significantly better for children in the fastest STS tertile compared to those in the mid $(P=0.06)$ and slowest tertiles. Age was also significant as a 
covariate $\left(P=0.001, \mathrm{P \eta}^{2}=.276, \beta=2.105\right)$. This study indicates that children who have better STS performance also have better FMS. These results support the STS test as a measure of functional motor competence in children aged 5-9 years.

D1.S3.1(3). The effect of a shortened sprint, reduced exertion high-intensity interval training (REHIT) protocol on affective response and peak oxygen uptake

\section{MATTHEW HAINES*}

\section{University of Huddersfield \\ *Corresponding author: m.haines@hud.ac.uk}

The minimum amount of exercise that has been shown to improve peak oxygen uptake $\left(\mathrm{V}_{2 \text { peak }}\right)$ is reduced-exertion, high-intensity interval training (REHIT) incorporating two $20 \mathrm{~s}$ maximal exertion cycle sprints within a 10-min exercise session (Metcalfe et al., 2011, European Journal of Applied Physiology, 112, 2767-2775). However, hedonistic theories of motivation propose that exercise above a certain intensity threshold results in physiological responses that negatively influence affective valence (i.e. increasing sensations of displeasure). This could lead to avoidance of the activity and poor exercise adherence. Given that sensation of exertion is a function of intensity and duration, it is plausible that reducing the time spent 'sprinting' may attenuate any negative affect associated with periods of high-intensity exercise. Accordingly, the aims of this study were to investigate affective response to a novel, shortened-sprint REHIT protocol; and to test the effect of this protocol on $\mathrm{VO}_{2 \text { peak. With }}$ institutional ethics approval, 24 men (age $21.2 \pm 1.9$ years; mass, $80.2 \pm 6.8 \mathrm{~kg}$; BMI, $25.5 \pm 1.8 \mathrm{~kg} \cdot \mathrm{m}^{2}$ ) were randomly assigned to one of two conditions: (1) shortened-sprint REHIT $(8 \times 5 \mathrm{~s}$ sprints), or $(2)$ traditional REHIT $(2 \times 20 \mathrm{~s}$ sprints). Participants completed 15 exercise sessions over a 5-7-week period, with $\dot{\mathrm{V}}_{2 \text { peak }}$ determined using a ramp cycling test pre- and post-intervention. Affect and rating of perceived exertion (RPE) were measured during exercise using the Feeling Scale (FS) and the 15-point Borg scale, respectively. Enjoyment was recorded 5-min after cessation of activity using the Exercise Enjoyment Scale. Factorial (condition $\times$ time) mixed ANOVA's were used to compare differences between groups. Affective valence was more favourable for shortened-sprint REHIT compared to traditional REHIT $(1.6 \pm 0.6$ vs. $0.2 \pm 1$ FS units, respectively; where 1 is 'fairly good' and 0 is 'neutral'; $P=0.001, d=$ 1.62). Similarly, peak RPE values were lower for shortenedsprint REHIT $(14.4 \pm 0.9$ vs. $16.2 \pm 1.1, P=0.001, d=-1.71)$. However, there was no difference in enjoyment. Compared to baseline, $\dot{\mathrm{V}}_{2 \text { peak }}$ increased in both groups ( $6 \%$ for shortened-sprint REHIT [ $d=-0.36$ ] and 9\% for traditional REHIT $[d=-0.53]$, both $P=0.01$ ), although there was considerable heterogeneity in training response within each group. The results suggest both conditions improve fitness without overly compromising cognitive and affective response, and as such may be genuinely time-efficient yet tolerable approaches to exercise. The challenge of translating this research into real-world practice remains.

D1.S3.1(4). Walking in short bouts throughout the day reduces postprandial triglycerides in older women with elevated fasting triglycerides

KYOKO KASHIWABARA ${ }^{1}$, TETSUHIRO KIDOKORO ${ }^{2}$, TAKUMA YANAOKA ${ }^{1,3}$, STEPHEN F. BURNS ${ }^{4}$, DAVID J. STENSEL $^{5} \&$ MASASHI MIYASHITA ${ }^{1 *}$

\section{${ }^{1}$ Waseda University, ${ }^{2}$ Juntendo University, ${ }^{3}$ Japan Society for the Promotion of Science, ${ }^{4}$ Nanyang Technological University, ${ }^{5}$ Loughborough University *Corresponding author: m.miyashita@waseda.jp}

Elevated non-fasting triglyceride (TG) concentrations are a causal risk factor for cardiovascular disease and all-cause mortality in men and women (Nordestgaard and Vardo, 2014, Lancet, 384, 626-635.). Most studies have reported that a single bout of continuous aerobic exercise ( $\geq 30 \mathrm{~min}$ ) reduces postprandial TG (Freese et al., 2012, Journal of Applied Physiology (1985), 116, 67-75), but little is known about the effect of multiple short ( $\leq 10 \mathrm{~min}$ ) bouts of exercise on postprandial TG in individuals at increased risk of cardiovascular disease. This study compared the effect of different patterns of walking on postprandial TG in postmenopausal women with elevated fasting TG. With institutional ethics approval, 12 inactive women (age: $71 \pm 5$ years) (mean $\pm \mathrm{s}$ ) with fasting TG $\geq 1.70 \mathrm{mmol} / \mathrm{L}$ completed three, one-day laboratory-based trials in a random order: (1) control, (2) continuous walking, and (3) multiple short bouts of walking. On the control trial, participants sat in a chair for $8 \mathrm{~h}$. For the walking trials, participants walked briskly in either one 30-min bout in the morning (09000930) or twenty $1.5 \mathrm{~min}$ bouts over $8 \mathrm{~h}$. Except for walking both exercise trials mimicked the control trial. In each trial, participants consumed standardised mixed macronutrient breakfasts (0800) and lunches (1100). Venous blood samples were collected in the fasted state and at 2, 4, 6 and $8 \mathrm{~h}$ after breakfast. The serum TG incremental area under the curve differed among trials $(12.7 \pm 5.8$ vs $8.2 \pm 3.1$ vs $8.5 \pm$ $5.4 \mathrm{mmol} \cdot 8 \mathrm{~h} / \mathrm{L}$, main effect of trial: Effect size $=0.459, P=$ $0.001)$ and was $35 \%$ and $33 \%$ lower with continuous $(95 \%$ Cl: -7.6 to $-1.6 \mathrm{mmol} \cdot 8 \mathrm{~h} / \mathrm{L}$, Effect size $=0.984, P=0.004)$ and multiple short bouts $(95 \% \mathrm{Cl}:-8.0$ to $-0.5 \mathrm{mmol} \cdot 8 \mathrm{~h} / \mathrm{L}$, Effect size $=0.762, P=0.024$ ) of walking than the control trial. There was no significant difference between the two walking trials $(P=1.000)$. These results indicate that accumulating $30 \mathrm{~min}$ of brisk walking in short $(1.5 \mathrm{~min}$ ) bouts is as effective in attenuating postprandial TG concentrations as the same volume of exercise taken in a continuous pattern. Our findings help substantiate recommendations in physical activity guidelines that similar health benefits can be obtained through accumulated versus continuous activity in at-risk older women but also suggest that bouts of activity of $<10$ min duration may impart benefit on selected cardiovascular disease risk markers. 
D1.S3.1(5). Comparative study of the effect of aquatic plyometric training versus land-based exercises on bone mineral density in physically active women

MANUEL A. RIVEROS, JOSE L. GARCIA, ADRIANA R. GUTIEREZ, CARLOS A. COLLAZOS, \& OSCAR F. RUBIANO*

\section{Manuela Beltrán University \\ *Corresponding author: oscar.rubiano@umb.edu.co}

Changes associated with a sedentary lifestyle are transforming the demographic and epidemiological profile worldwide (Vos et al., 2013). The sedentary lifestyle has been associated with increased incidence of chronic noncommunicable diseases including osteoporosis (Tremblay, Colley, Saunders, Healy, \& Owen, 2010). Due to related adverse health outcomes, osteoporosis is considered as a major public health burden (Dempster, 2011; Hernlund et al., 2013). The effects of physical activity (PA) on bone mineral density (BMD) are controversial and results seem to depend on the influence of multiple factors (Sinaki, 1996; Whitfield, Kohrt, Gabriel, Rahbar, \& Kohl III, 2015). Different studies suggest that low and high impact exercise increase BMD (Grove \& Londeree, 1992; Zhao, Zhao, \& Zhang, 2014). Plyometric jump training is considered a high-impact activity which has been related to increments in BMD (Guadalupe-Grau, Fuentes, Guerra, \& Calbet, 2009; Vainionpää et al., 2006; Witzke \& Snow, 2000). Aquatic plyometric training (APT) may arise as a new intervention alternative to non-controlled land-based physical activity program (LBPAP). The aim of this study was to determine the effect of APT on BMD, compared to LBPAP in physically active women. Using a non-probabilistic intentional sampling, data was we collected from a population 102 women. Only 48 women met the inclusion criteria: Age 60 \pm 5 years, physically, no less than 2 years, active according to the American College of Sports Medicine guidelines and, a non-pharmacologic treatment for osteopenia/osteoporosis different from calcium. The study was approved by the Manuela Beltrán university ethics committee. Participants were randomly assigned to each group. The study group $(n=24)$ performed a 22-weeks APT program, 6 of which corresponded to a water adaptation process. Participants trained $1 \mathrm{~h} 3$ times per week; control group performed their regular non-controlled LBPAP. When groups were compared, a non-statistical difference was revealed for the different variables measured (DMO, T-score, and Z-score). The ANOVA analysis revealed significant differences for DMO site $(F=195.5 ; P<0.05)$ but, not significant differences between groups for DMO, site for group, nor group for site for period of assessment in this study $(P>0.05)$. These results suggested that exercises programs designed to maintain or improve DMO should include a combination of moderate to high-impact exercises. Thus, the risk of fractures associated with falls in elderly population may be reduced due to protective effect of the muscle-skeletal system.
D1.S3.1(6). Association between breakfast frequency and physical activity and sedentary time during different times of the day in children from 12 countries

JULIA K. ZAKRZEWSKI-FRUER ${ }^{*}$, FIONA B. GILLISON ${ }^{2}$, SEAN CUMMING ${ }^{2}$, PETER T. KATZMARZYK ${ }^{3}$, STEPHANIE T. BROYLES ${ }^{3}$, CATHERINE M. CHAMPAGNE ${ }^{3}$, JEANPHILIPPE CHAPUT ${ }^{4}$, KARA D. DENSTEL ${ }^{3}$, MIKAEL FOGELHOLM ${ }^{5}$, GANG HU ${ }^{3}$, REBECCA KURIYAN ${ }^{6}$, ANURA V. KURPAD ${ }^{6}$, ESTELLE V. LAMBERT $^{7}$, CAROL MAHER ${ }^{8}$, JOSÉ MAIA ${ }^{9}$, VICTOR MATSUDO ${ }^{10}$, EMILY F. MIRE ${ }^{3}$, TIM OLDS $^{8}$, VINCENT ONYWERA ${ }^{11}$, OLGA L. SARMIENTO ${ }^{12}$ MARK S. TREMBLAY ${ }^{4}$, CATRINE TUDOR-LOCKE ${ }^{3,13}$, PEI ZHAO $^{14}$ \& MARTYN STANDAGE ${ }^{2}$

${ }^{1}$ University of Bedfordshire, ${ }^{2}$ University of Bath,

${ }^{3}$ Pennington Biomedical Research Center, ${ }^{4}$ Children's Hospital of Eastern Ontario Research Institute, ${ }^{5}$ University of Helsinki, ${ }^{6}$ St Johns Research Institute, ${ }^{7}$ University of Cape Town, ${ }^{8}$ University of South Australia, ${ }^{9}$ University of Porto, ${ }^{10}$ Centro de Estudos do Laboratório de Aptidão Física de São Caetano do Sul (CELAFISCS), ${ }^{11}$ Kenyatta University, ${ }^{12}$ Universidad de los Andes, ${ }^{13}$ University of Massachusetts Amherst, ${ }^{14}$ Tianjin Women's and Children's Health Center *Corresponding author: Julia.Fruer@beds.ac.uk

More frequent breakfast consumption has been associated with higher self-reported physical activity (PA) in children (Sandercock, Voss and Dye, 2010, European Journal of Clinical Nutrition, 64, 1086-1092). However, objective measures of PA have not provided clear evidence of an association with breakfast frequency (Corder et al., 2011, British Journal of Nutrition, 105, 316-321; Corder et al., 2014, American Journal of Clinical Nutrition, 99, 361-368). Furthermore, this research is based on children from highincome countries with limited geographic and socio-cultural variability. Using data from the International Study of Childhood Obesity, Lifestyle and the Environment, this study examined associations between breakfast frequency and objectively-measured PA and sedentary time during different times of the day in 9-11 year olds from 12 countries. With institutional ethics approval from each study site, habitual breakfast frequency (days/week) was assessed via questionnaire and PA and sedentary time were assessed over seven consecutive days using an accelerometer in 6228 children from Australia, Brazil, Canada, China, Colombia, Finland, India, Kenya, Portugal, South Africa, the United Kingdom and the United States. Accelerometry data was partitioned into morning (wake time to $<1200 \mathrm{~h}$ ) and afternoon (1200 h to <bed time) time segments. Multilevel statistical models were used to account for the hierarchical nature of the data, adjusting for age, sex, parental education, body mass index (BMI) $z$-score and accelerometer wear time. In the morning, sedentary time was higher in rare breakfast consumers compared with occasional $(P=0.048)$ and frequent $(P=$ $0.012)$ breakfast consumers. Morning light PA (LPA) $(P=$ $0.031)$ and moderate-to-vigorous PA (MVPA) $(P=0.030)$ 
were lower in rare breakfast consumers compared with frequent breakfast consumers. Breakfast frequency was not associated with afternoon sedentary time or PA. For the whole day, the higher sedentary time in rare than frequent breakfast consumers neared significance $(P=$ 0.053 ) and LPA was lower in rare compared with occasional breakfast consumers $(P=0.042)$. No associations were found between breakfast frequency and total daily MVPA. No interactions with study site were found. In a multinational sample of children, rarely consuming breakfast was associated with more time spent sedentary and less time in LPA and MVPA during the morning, but not during the afternoon, independent of age, sex, parental education and BMI z-score. These associations for the morning contributed to the higher sedentary time and lower time spent in LPA across the whole day in rare breakfast consumers. Further research is required to understand the potential role of increased breakfast frequency in PA promotion in globally representative samples of children. 


\section{Day 1. Free Communications - Physiology and Nutrition}

D1.S3.2(1). A comparison of neural adaptations after twelve weeks and four years of resistance training

THOMAS G. BALSHAW ${ }^{1,2^{*}}$, GARRY J. MASSEY ${ }^{1,2}$, THOMAS M. MADEN-WILKINSON ${ }^{3}$, MARCEL B. LANZA ${ }^{2}$ \& JONATHAN P. FOLLAND ${ }^{1,2}$

\section{${ }^{1}$ Arthritis Research UK Centre for Sport, Exercise and Osteoarthritis, ${ }^{2}$ Loughborough University, ${ }^{3}$ Sheffield Hallam University \\ ${ }^{*}$ Corresponding author: t.g.balshaw@lboro.ac.uk @tombalshaw}

It is unclear whether several years of resistance training (RT) causes continued neural adaptations in agonist and antagonist activation, and specifically how the inter-relationships between these two variables and muscular torque changes, beyond those documented by short-term intervention studies. Whilst a longitudinal study of several years duration may be impractical, a comparison of groups who have completed distinct durations of RT may facilitate a cross-sectional investigation of the time course of these adaptations. The purpose of this investigation was to conduct a comparison of agonist and antagonist activation throughout the range of voluntary torque between groups of different RT experience. Fifty-seven young, healthy men (untrained [UNT] $\mathrm{n}=29$, short-term RT [12WK] $\mathrm{n}=14$, and long-term RT [mean of 4-years; 4YR] $n=14$ ) performed maximum and submaximum $(20,40,60,80 \%)$ unilateral isometric knee extension contractions with torque, quadriceps (QEMG) and hamstring (HEMG) surface EMG recorded during all contractions. The following variables were also measured: maximum Quadriceps anatomical cross sectional area (QCSA; via MRI); and subcutaneous tissue thickness (SCT; primarily subcutaneous fat; via B-mode ultrasonography) at the sites where QEMG sensors were positioned. QEMG was corrected to account for the known covariate of SCT. Maximum voluntary torque (MVT) for 4YR (407 $\pm 63 \mathrm{Nm}$ ) was greater than UNT (255 $\pm 42 \mathrm{Nm})$ and $12 \mathrm{WK}(293 \pm$ $49 \mathrm{Nm}$ ), respectively ([both] Least Significant Difference [LSD] $P<$ 0.001 ), whilst $12 \mathrm{WK}$ was stronger than UNT (LSD $P=0.021$ ). QACSA of 4YR $\left(137.7 \pm 13.9 \mathrm{~cm}^{2}\right)$ was greater than $12 \mathrm{WK}(97.2$ $\left.\pm 15.3 \mathrm{~cm}^{2}\right)$ and UNT $\left(91.6 \pm 12.1 \mathrm{~cm}^{2}\right)$, respectively, ([both] LSD $P<0.001)$, but did not differ between 12WK and UNT $(P=0.204)$. QEMG during isometric MVT was greater for 4 YR $(0.277 \pm 0.060$ $\mathrm{mV}$ ) and 12WK (0.255 $\pm 0.061 \mathrm{mV})$ compared to UNT (0.192 \pm $0.042 \mathrm{mV}$; [both] LSD $P<0.001$; ES = 1.29-1.75 [both] "large"), but did not differ between the two RT groups $(P=0.282$, ES $=$ 0.36 "small"). The slope of the torque-QEMG relationship did not differ between groups (ANOVA, $P=0.107$ ), but the slope of the HEMG-QEMG relationship was lower for both 4YR $(54 \pm 44)$ and $12 \mathrm{WK}(83 \pm 41)$ compared to UNT $(132 \pm 72$; LSD $0.001<P \leq$ 0.028 ); with 12 WK's relationship occupying a distinct and intermediate position between the two other groups. In conclusion, long-term RT does not appear to induce further increases in agonist neural drive during isometric MVT production when compared to short-term RT, but inter-muscular coordination, specifically reduced antagonist co-activation, continues to adapt beyond 12 weeks of RT.

\section{D1.S3.2(2). Exercise-induced muscle damage and time-course of recovery in young and middle-aged males}

\section{JOHN F. T. FERNANDES ${ }^{1,2^{*}}$, KEVIN L. LAMB ${ }^{2}$ \& CRAIG TWIST $^{2}$
${ }^{1}$ Hartpury College, ${ }^{2}$ University of Chester
*Corresponding author: j.fernandes@chester.ac.uk @JFTFsport

Resistance training provides a potent method to offset the age-associated losses in strength and muscle mass (Roth et al., 1999, Journal of Applied Physiology, 86, 1833-1840). However, the recovery from muscle damage caused by resistance training in middle-aged males remains unknown. This study compared the time course of recovery after heavy volume lower limb resistance exercise in trained young $(n=9 ;$ mean $\pm s$ age $22.3 \pm$ 1.7 years) and trained ( $n=9 ; 39.9 \pm 6.2$ years) and untrained ( $n=9$; age $44.4 \pm 6.3$ years) middle-aged males. With institutional ethics approval, participants completed measurements of muscle soreness, plasma creatine kinase, maximal voluntary contraction, voluntary activation and resting twitch torque of the knee extensor and peak power during squatting at 20 and $80 \%$ onerepetition maximum (1RM) before and at 24 and $72 \mathrm{~h}$ after muscle-damaging exercise comprising $10 \times 10$ squats at $60 \% 1 \mathrm{RM}$. Mass and fat-mass were not significantly different for any of the comparisons $(P>0.05)$ though fat-free was lower in trained middle-aged males (63.9 \pm $6.5 \mathrm{~kg})$ compared to the young males $(71.4 \pm 7.9 \mathrm{~kg} ; P<$ $0.05)$, but not different to their untrained counterparts $(68.6 \pm 7.1 \mathrm{~kg} ; P>0.05)$. No differences in squat $1 \mathrm{RM}$ were observed between the young $(130.8 \pm 26.8 \mathrm{~kg})$, trained middle-aged $(109.3 \pm 22.5 \mathrm{~kg})$ and untrained middle-aged $(98.4 \pm 14.25 \mathrm{~kg})$ groups $(P>0.05)$. Power $(640.2$ $\pm 207.3 \mathrm{~W}$ ) produced by the trained middle-aged group $(603.2 \pm 162.6 \mathrm{~W})$ during the muscle-damaging exercise was not different $(P>0.05)$ to the trained young $(770.4 \pm$ $278.4 \mathrm{~W}$; effect size $(E S)=0.78)$, or untrained middle-aged groups $(547.0 \pm 75.0 \mathrm{~W}$; ES $=0.47)$. Muscle soreness and creatine kinase were increased in all groups at 24 and 72 $\mathrm{h}$ after muscle-damaging exercise $(P<0.05)$. All measures of voluntary and evoked muscle function were impaired at $24 \mathrm{~h}(P<0.05)$, with only peak power during squatting at $80 \% 1 \mathrm{RM}$ and resting twitch recovered at $72 \mathrm{~h}$ (both $P>0.05)$. There were no significant group $x$ time interactions for any of the comparisons $(P>0.05$; ES $=-0.40$ to 0.28 and -0.82 to 0.91 for the trained and middle-aged comparisons, respectively). These data indicate that regardless of training experience, the muscle damage response and time-course of recovery after heavy volume resistance exercise is similar for young and middle-aged males. Accordingly, young and middle-aged males can programme their resistance training in a similar fashion. 
D1.S3.2(3). Elevated baseline work-rate slows pulmonary oxygen uptake kinetics, decreases critical power and increases $W^{\prime}$ during supine cycle exercise

\section{RICHIE GOULDING*, DENISE ROCHE \& SIMON MARWOOD}

\section{Liverpool Hope University}

${ }^{*}$ Corresponding author: gouldir@hope.ac.uk @RichieGoulding1

Critical power (CP) and $\mathrm{W}^{\prime}$ are fundamental parameters in defining severe-intensity exercise tolerance; however their physiological determinants are unclear. $\mathrm{CP}$ is related to the phase II time constant of oxygen uptake kinetics ( $\left.\tau V_{\mathrm{O}_{2}}\right)$, whereas $\mathrm{W}^{\prime}$ is related to the magnitude of the oxygen uptake $\left(\mathrm{VO}_{2}\right)$ slow component (SC) (Murgatroyd et al., 2011, Journal of Applied Physiology, 110, 1598-1606). Initiating exercise from a moderate-intensity baseline increases $\mathrm{\tau} \dot{V}_{\mathrm{O} 2}$ and reduces the $\mathrm{VO}_{2}$ SC (DiMenna et al., 2010, Journal of Applied Physiology, 109, 1148-1154), whereas exercising in the supine position impairs oxygen delivery to the active muscles, increases $\tau \dot{V}_{02}$, and increases the magnitude of the SC (Koga et al., 1999, Journal of Applied Physiology, 87, 253-260). Therefore, the purpose of this study was to determine the impact of baseline work-rate on pulmonary $\mathrm{VO}_{2}$ kinetics, $\mathrm{CP}$ and $\mathrm{W}^{\prime}$ during supine exercise. With institutional ethical approval, 8 healthy men completed an incremental ramp test to determine maximal oxygen uptake $\left(\mathrm{VO}_{2} \max \right)$ and the gas exchange threshold (GET). This was followed by 8 visits whereby $C P, W^{\prime}$, pulmonary $\mathrm{VO}_{2}$ and muscle deoxyhaemoglobin $\left(\tau_{[\mathrm{HH} b]}\right.$; via near-infrared spectroscopy) kinetics were determined via 4 exhaustive severe-intensity constant load exercise trials performed in the supine position in two conditions: (1) exercise initiated from an unloaded baseline $(U>S)$ and (2) exercise initiated from a moderate-intensity baseline at $90 \% \mathrm{GET}(\mathrm{M}>\mathrm{S})$. $\mathrm{M}>$ $S$ reduced $C P(U>S=146 \pm 39 \mathrm{~W}$ vs. $\mathrm{M}>\mathrm{S}=132 \pm 33 \mathrm{~W} ; P$ $=0.02)$ and increased $W^{\prime}(U>S=11.5 \pm 3.4 \mathrm{~kJ}$ vs. $M>S=$ $16.4 \pm 5.3 \mathrm{~kJ} ; P=0.016)$ compared to $\mathrm{U}>\mathrm{S}$. There was a concomitant increase in $\mathrm{\tau} \dot{V}_{\mathrm{O} 2}(\mathrm{U}>\mathrm{S}=45 \pm 16 \mathrm{~s}$ vs. $\mathrm{M}>\mathrm{S}=$ $69 \pm 29 ; P<0.01)$, reduction in the $\dot{V} \mathrm{O}_{2} \mathrm{SC}(U>S=0.22 \pm$ 0.13 L.min $^{-1}$ vs. $M>S=0.10 \pm 0.10$ L.min ${ }^{-1} ; P<0.01$ ) and increase in $\tau_{[H H b]}(U>S=15 \pm 5 \mathrm{~s}$ vs. $M>S=43 \pm 11 \mathrm{~s} ; P<$ $0.01)$ in $\mathrm{M}>\mathrm{S}$ compared to $\mathrm{U}>\mathrm{S}$. The concomitant increase

in $\tau \dot{V}_{\mathrm{O} 2}$ and reduction in $\mathrm{CP}$ suggest that $\mathrm{\tau} \dot{V}_{\mathrm{O} 2}$ is an independent determinant of $\mathrm{CP}$; the greater $\tau_{[\mathrm{HHb}]}$ in $M>S$ compared to $U>S$ suggests that this effect was unrelated to oxygen availability. The concomitant reduction in $\mathrm{CP}$ and increase in $\mathrm{W}^{\prime}$ in $\mathrm{M}>\mathrm{S}$ compared to $\mathrm{U}>\mathrm{S}$ provides further evidence of the inter-dependence of these two parameters.
D1.S3.2(4). Catecholamines, but not sit-up tilt, are predictive of the inflammatory response to a wheelchair half-marathon

SVEN HOEKSTRA ${ }^{1 *}$, YOSHI-ICHIRO KAMIJO ${ }^{2}$, TOKIO KINOSHITA ${ }^{2}$, BEN STEPHENSON ${ }^{1}$, NICOLETTE BISHOP ${ }^{1}$, CHRISTOF LEICHT ${ }^{1}$, VICKY TOLFREY ${ }^{1} \&$ FUMIHIRO TAJIMA ${ }^{2}$

\section{${ }^{1}$ Loughborough University, ${ }^{2}$ Wakayama Medical University *Corresponding author: s.p.hoekstra@lboro.ac.uk @sven_hoekstra}

The beneficial inflammatory response to upper-body exercise seems attenuated in individuals with a cervical spinal cord injury (CSCl), possibly due to autonomic dysfunction and a blunted catecholamine response to exercise (Paulson et al., 2013, Medicine and Science in Sports and Exercise, 45, 1649-1655). To test this hypothesis, this study investigated the influence of autonomic function and catecholamines on the inflammatory response to a wheelchair half-marathon. With institutional ethics approval, 17 male recreationally trained wheelchair athletes with $\mathrm{CSCl}(\mathrm{N}=7, \mathrm{CSCl})$, paraplegia or another impairment $(\mathrm{N}=10$, NON-CSCl) participated in a wheelchair half-marathon following a sit-up tilt test for the assessment of autonomic function. Outcome measures for the tilt test, which was conducted 1 or 2 days prior to race-day, were orthostatic hypotension (defined as a drop in systolic blood pressure of $>20 \mathrm{mmHg}$ after the onset of tilt manoeuvre), the ratio of power in the low and high frequency domains of the RR interval variability (LF/HF RRI) and power in the low frequency domain of systolic blood pressure variability (LF SBP) in the supine position. On race-day, blood was taken prior, immediately post and $1 \mathrm{~h}$ post-race to determine serum levels of adrenaline, noradrenaline and interleukin (IL)-6. Orthostatic hypotension was apparent in $4 \mathrm{CSCl}$ and 1 NON-CSCl athletes, while CSCl showed a lower LF/HF RRI $(P=$ 0.038 , ES $=0.31)$ and LF SBP $(P=0.005$, ES $=0.49)$ compared to $\mathrm{NON}-\mathrm{CSCl}$. In response to the race, adrenaline and noradrenaline increased only in NON-CSCI $(P<0.005, \mathrm{ES}>0.61)$. Although both groups showed elevated IL-6 levels post-race $(P<0.033$, ES $>$ $0.63)$, the increase was larger in NON-CSCI $(P=0.040, E S=0.29)$. Post-race catecholamine levels together explained $60 \%$ of the variance in the IL- 6 response for the complete group $(r=0.77, P$ $=0.040$ ). Although LF/HF RRI was a significant predictor of postrace adrenaline levels $\left(\mathrm{R}^{2}=0.29, r=0.54, P=0.04\right), \mathrm{LF} / \mathrm{HF}$ RRI and LF SBP did not explain the variance in post-race IL-6 levels $(P>$ $0.271)$. In conclusion, a sit-up tilt test revealed differences in autonomic function between CSCl and NON-CSCl. However, while post-race catecholamine concentrations were highly correlated with post-race IL-6 levels, autonomic function did not predict the inflammatory response to the race. Therefore, this study showed that although the catecholamine response to exercise is dependent on autonomic function, post-race catecholamine levels, but not autonomic function, are predictive of the inflammatory response to a wheelchair half-marathon. 
D1.S3.2(5). Acute effects of low-load systemic and localised hypoxic resistance training on skeletal muscle morphology, metabolic stress and muscle tissue oxygenation

\section{RYAN MARSHALL, WILLIAM D. SHELDON \& ELISABETH M. BOARD*}

\section{University of Sunderland \\ *Corresponding author: lisa.board@sunderland.ac.uk}

Traditional resistance training (RT) with loads exceeding $80 \%$ of 1 repetition maximum (1RM) are commonly prescribed to increase gains in muscular strength and size (Kraemer et al., 2002). High intensity RT however may not be a viable option for a number of potential populations. Reducing inspired oxygen or arterial blood flow to the muscle creates an intramuscular hypoxic environment (Scott et al., 2014). Hypoxic resistance training therefore has been postulated to increase skeletal muscle adaptations similar to, if not greater than, that of traditional resistance training (Scott et al., 2015). This has been shown to enhance physiological adaptations to both skeletal (Fry et al., 2010) and vascular tissues (Hunt et al., 2013). The aim of the study was to investigate the acute effects of low-load resistance training $(30 \%$ 1RM) with either a systemic (SH-RT) or localised hypoxic (LH-RT) stimulus. Concurrent bilateral elbow flexion and elbow extension exercises were performed (4 sets of $30,15,15$ and 15 repetitions with $30 \mathrm{~s}$ intra-set rest). SH-RT were subject to a systemic hypoxic dose of $\mathrm{FIO2}=0.100$, LHRT $60 \%$ estimated arterial occlusion pressure (AOP) and control (CON) $\mathrm{FIO} 2=0.209$ with no cuff inflation. During the experiment muscle thickness (MTH), blood lactate ([BLa]) and muscle tissue oxygenation (StO2) were measured. Our study showed LH-RT induced the greatest increase in elbow flexor MTH (18.7\%) compared to SH-RT (7.7\%) and CON (8.3\%) with no difference in elbow extensor MTH $(P=$ $0.220)$. LH-RT showed the greatest increase in [BLa] $\left(7.9 \mathrm{mmol} / \mathrm{L}^{-1}\right)$ compared to SH-RT $\left(4.8 \mathrm{mmol} / \mathrm{L}^{-1}\right)$ and $\mathrm{CON}$ $\left(3.8 \mathrm{mmol} / \mathrm{L}^{-1}\right)(P=0.001)$. Elbow flexor $\mathrm{StO} 2$ was significantly decreased in both LH-RT $(P<0.001)$ and SH-RT $(P=$ $0.017)$ during intra-set recovery period compared to CON. The LH-RT significantly decreased elbow extensor $\mathrm{StO} 2$ compared to SH-RT $(P=0.005)$ and CON $(P=0.004)$, and no difference was observed between SH-RT and CON $(P>0.05)$. In conclusion, localised hypoxic resistance training by way of moderate restriction of blood flow to the upper limbs induces greater physiological stress and potential anabolic adaptations than that of systemic hypoxia. On a cautionary note, severe delayed onset muscle soreness (DOMS) was reported by each of the LH-RT and SH-RT participants up to $48 \mathrm{~h}$ post exercise. Future work should look to determine the molecular metabolic pathways of BFR-RT as a potential therapy in age and disease related muscle atrophy.
D1.S3.2(6). The effects of crank rate on the physiological and perceptual responses to high intensity intermittent upper body exercise

\section{MIKE PRICE $^{1 *}$, PAUL SMITH ${ }^{2} \&$ GABRIEL GENNUSO ${ }^{1}$ \\ ${ }^{1}$ Coventry University, ${ }^{2}$ Cardiff Metropolitan University \\ *Corresponding author: mike.price@coventry.ac.uk}

The effects of crank rate on submaximal and peak physiological responses to arm crank ergometry have been evaluated (Smith et al., 2001, Journal of Sports Sciences, 19, 955-960). However, much less information is available regarding the effects of crank rate on high intensity intermittent arm exercise. Therefore, the aim of this study was to examine the effects of crank rate on the physiological and perceptual responses to high intensity interval training (HIIT) type exercise during arm-crank ergometry (ACE). Following University ethics approval eight male participants (mean \pm SD age: $21.9 \pm 0.8$ years, stature: $1.81 \pm 0.08 \mathrm{~m}$; body mass: $78.1 \pm 9.9 \mathrm{~kg}$ ) undertook three exercise trials on an electronically braked arm crank ergoemter (Lode, Angio); an incremental ACE protocol to determine peak minute power $(118 \pm 34 \mathrm{~W})$ and peak oxygen uptake $(2.46 \pm 0.56$ L. $\mathrm{min}^{-1}$ ) at a crank rate of 70 rev. $\mathrm{min}^{-1}$ and two further trials involving HIIT at crank rates of either 50 rev. $\mathrm{min}^{-1}$ or 90 rev. $\mathrm{min}^{-1}$. The HIIT consisted of ten alternating $2 \mathrm{~min}$ bouts of ACE at $75 \%$ and $25 \%$ of peak minute power (i.e. $20 \mathrm{~min}$ in total). Heart rate (Polar heart rate monitor) and expired gases (Metamax, 3b) were continually monitored and averaged in the last $30 \mathrm{~s}$ of each segment along with ratings of perceived exertion (Borg Scale) for central (cardiorespiratory) and local (working muscles) sensations. Blood samples were collected from an earlobe at rest and on the completion of each $75 \%$ peak minute power bout for analysis of blood lactate concentration (Biosen, C_Line). Data were analysed by two-way (crank rate $\times$ time) repeated measures analysis of variance (SPSS, v24). Significant main effects for crank rate were observed for oxygen consumption $(P<0.01 ; \mathrm{ES}=0.790)$ and ventilation rate $(P<0.05$; ES $=0.753$ ) with both variables being greater during 90 rev. $\mathrm{min}^{-1}$ (mean across trials: $1.64 \pm 0.36$ and $48.9 \pm 7.0 \mathrm{~L}$. $\mathrm{min}^{-1}$ respectively) when compared to 50 rev. $\mathrm{min}^{-1}$ (1.22 \pm 0.25 and $40.6 \pm 6.4$ L. $\mathrm{min}^{-1}$, respectively). No differences were observed between crank rates for heart rate, blood lactate concentration or local or central ratings of perceived exertion $(P>0.05$; ES $=0.295,0.983,0.090,0.245$, respectively). The greater respiratory responses and similar anaerobic responses suggest that the aerobic energy system per se may be more sensitive to differences in crank rate at the exercise intensities studied. Greater intensities of exercise may yield more discriminatory responses in heart rate, blood lactate concentration and ratings of perceived exertion. 


\section{Day 1. Free Communications - Psychology (Session 1)}

\author{
D1.S3.3(1). The motivation of elite athletes: a \\ mental health perspective

\section{RACHEL B. SHEEHAN*, MATTHEW P. HERRING \& MARK J. CAMPBELL}

\author{
University of Limerick \\ *Corresponding author: rachel.sheehan@ul.ie \\ @rachelbsheehan
}

Motivation has been the subject of much research in the literature, whereas athlete mental health has received limited attention. Motivational complexities in elite sport (e.g., extreme emphasis on winning) are somewhat reflected in the mental health literature, where there is evidence for both protective and risk factors for athletes. Notably, few previous studies have linked the why of behaviour to mental health. Against this backdrop, the objectives of this study were, firstly, to characterise the motivation and mental health of elite athletes, and, secondly, to examine the relationships between motivationrelated and mental health variables. With institutional ethics approval, 215 elite athletes (mean age $=22.84$ \pm 4.11 years) from 11 teams completed eight psychometric inventories (Sport Motivation Scale II, Task and Ego Orientation in Sport Questionnaire, Basic Need Satisfaction in Sport Scale, Perceived Motivational Climate in Sport Questionnaire II, Profile of Mood States - Brief, Quick Inventory of Depressive Symptomatology Self Report, Pittsburgh Sleep Quality Index, State-Trait Anxiety Inventory - Y2). As well as providing descriptive statistics and correlations, and examining the reliability and validity of the scales, structural equation modelling was used to investigate the relationships between variables. Though our sample displayed predominantly positive motivational patterns, the mental health results were mixed (e.g., 45\% with mild-to-moderate depressive symptoms, $42 \%$ poor sleepers). Examination of the fit statistics indicated that the model fit the data well: $X^{2}(26)=$ 30.143, $P=0.2617, \mathrm{CFI}=0.997, \mathrm{TLI}=0.985, \mathrm{RMSEA}=$ 0.027]. The model reinforced previously reported relationships between motivation-related variables, with motivational climate influencing basic needs, which in turn affect motivation. Uniquely, the model is the first to show how four mental health outcomes are associated with motivational constructs. Specifically, an ego climate was positively associated with depressive symptoms, sleep quality, and trait anxiety, while competence had an inverse relationship with trait anxiety. Furthermore, total mood disturbance, depressive symptoms, and sleep quality predicted non-self-determined forms of motivation. In addition to characterising the motivation and mental health of a sample of elite athletes, our study is the first of its kind to suggest that poor mental health can be detrimental to motivation. These findings point to the importance of monitoring athlete mental health as an outcome in and of itself, but also as an antecedent to motivation. In elite sport, optimising the well-being and drive of athletes is essential for performance, and our model provides potential avenues for doing this.

D1.S3.3(2). "You have to be mental to jump off a board any way": elite divers' conceptualizations and perceptions of mental health

\section{MELISSA COYLE ${ }^{1 *}$, PAUL GORCZYNSKI ${ }^{2} \&$ KASS GIBSON $^{1}$}

\section{${ }^{1}$ Plymouth Marjon University, ${ }^{2}$ University of Portsmouth *Corresponding author: mcoyle@marjon.ac.uk @mcsportpsych}

Mental health research in elite sport focuses predominantly on mental illness prevalence rates and help-seeking behaviours. Diving has been identified as a sport that generates particular challenges for maintaining mental health, yet has received scant attention from researchers. Therefore, the purpose of this study explores what mental health, experiences of mental health, and mental health related behaviours mean for a group of young, elite athletes as conditioned by their, perhaps perceived atypical, social context as elite athletes. Method: Semistructured interviews were conducted with purposively sampled eight elite divers aged between 14 and 24 years with between 5 and 16 years of diving experience who have competed in international level diving competitions including Olympic, Common Wealth and World Cup competitions. Interviews were recorded, transcribed verbatim, and analysed using inductive thematic analysis. Results: Analysis identified mental health literacy, experiences of mental health, risk factors, and opportunities for support as themes. Mental health generated negative connotations for participants attributable to knowledge development occurring through personal and vicarious experience of mental illness. Limited knowledge of symptoms of mental illnesses was evident demonstrating low levels of mental health literacy. Participants identified a range of risk-factors inherent in their sport performance and culture, as supported by this direct quote "You have to be mental to jump off a board anyway", in relation to potential development and exacerbation of mental health illness. Conclusions: Our results indicate the need to recognize the performative nature of mental health for elite divers and therefore social and cultural influences alongside biophysical. Greater efforts need to be made to help improve the overall mental health literacy of elite divers so that with great knowledge of mental health illness they may be able to seek and adhere to the relevant support and treatment where required. 
D1.S3.3(3). Wellbeing of practitioners travelling with athletes: balancing between sanity and insanity

\section{KOTRYNA FRASER $^{1 *} \&$ MOJI SHAHVAIL ${ }^{2}$}

\section{${ }^{1}$ The University of Edinburgh, ${ }^{2}$ The Pennsylvania State University \\ *Corresponding author: kotryna.fraser@ed.ac.uk @coach_kotryna}

Sport and exercise psychology practitioners are often expected to travel with a team or an individual athlete to various camps and competitions as a part of needs analysis or delivery of their services (Terry, 2010, In Hanrahan \& Andersen (Eds.) Routledge handbook of applied sport psychology (pp. 345-354). Abingdon: Routledge). Significant amount of research has focused on distinct issues related to athletes' travel (e.g., nutrition or transportation of equipment) or conducting needs assessment within different environments (e.g., away game versus individual sessions). However, little research has been conducted investigating practitioners' approaches to providing services when travelling with their clients, and practitioners' overall wellbeing. Therefore, the aim of this presentation is to introduce and reflect on practitioner's diverse experiences when travelling with two different clientele groups - a teenage motorsport racer and an age-group field hockey team. In both instances travel was conducted as part of needs analysis to observe athletes' coping skills, performing under pressures and interacting with other team members while being away from home. In both case studies, social occasions (e.g., eating together) were utilised for building trust and rapport, and to address problematic areas, for example, lack of commitment (Watson II \& Shannon, 2010, In Hanrahan \& Andersen (Eds.) Routledge handbook of applied sport psychology (pp. 90-100). Abingdon: Routledge). However, intense travel experiences presented additional issues to the practitioner which had to be immediately addressed. These included: being available and being observed almost 24/7, continuously resolving interpersonal conflicts between athletes and providing support for both athletes' and coaching staff. These issues caused mental fatigue and increased self-awareness for prolonged periods of time, possibly impacting practitioner's judgement, decision making and overall mental wellbeing (Rogers, 2004, Coaching skills: A handbook. Maidenhead: Open University Press). The importance of sound physical and psychological preparation of practitioners before and during travel will be discussed in light of practitioner's self-reflections and debrief during their peer-mentoring process. Overall, such dynamic travel experiences ought to be used not only as a means for on-going needs analysis and building rapport with athletes, but also as a developmental opportunity for practitioners. Making sure the practitioners are equipped with necessary professional skills and competence (e.g., effective management of resources) will help to maintain quality services when working under difficult conditions and promote their overall wellbeing when 'hitting the road' with their clients.

\section{D1.S3.3(4). A brief sports-based mental health literacy programme to increase help-seeking among adolescent male athletes: a cluster- randomised controlled trial}

\section{SARAH LIDDLE*, FRANK DEANE \& STEWART VELLA}

\section{University of Wollongong}

*Corresponding author: skl697@uowmail.edu.au

Mental illnesses are a growing concern among young people. Most disorders have their onset during this period, and around $14 \%$ of all Australian young people aged 12-17 years experience a mental illness in a 12-month period. Critically, more than a third of Australian adolescents who experience a mental illness do not access health services, with the most common reasons relating to stigma or poor mental health literacy (Lawrence et al., 2015, Department of Health, Canberra). Sport participation and physical activity can provide many positive physical and psychosocial health benefits and sports participation may be a protective factor against the development of mental illness. A large proportion of young people participate in organised sport each year and this presents an opportunity for mental health promotion. Therefore, the aim of this study was to assess the effects of a brief, sports-based mental health literacy programme to increase help-seeking among adolescent male athletes. A 45-min Help Out a Mate (HOAM) workshop was developed and its effects studied in a cluster-randomised controlled trial. After consenting to participate, teams were randomised to either the HOAM group ( $n=$ 47) or a wait-list control group $(n=55)$. Participants completed a survey at three time-points: baseline, immediately post-intervention, and at one-month follow-up. After consenting to participate, teams were randomised to either the HOAM group $(n=47)$ or a wait-list control group ( $n=$ 54). Results indicated that compared to a wait-list control, participants in the HOAM condition had significantly increased knowledge of the signs and symptoms of common mental illnesses, intentions to provide help to a friend who may be experiencing a mental health problem, and attitudes that promote recognition and help-seeking. Confidence, personal help-seeking intentions and psychological distress did not significantly change between groups over time. The results suggest that even a very brief intervention can be effective in improving the mental health literacy of young males in a sport environment, and may promote early intervention and referral of young athletes with mental health concerns to appropriate adults or professionals. 


\section{D1.S3.3(5). Development and qualitative} evaluation of a website and mobile-app supported intervention to increase resilience and wellbeing in youth sport

\section{CHRISTIAN SWANN ${ }^{1 *}$, MICHAEL NOETEL ${ }^{2}$, ANDREA FOGARTY ${ }^{3,4}$, RICHARD KEEGAN ${ }^{5}$, KATHERINE \\ TAMMINEN ${ }^{6}$, MUSTAFA SARKAR ${ }^{7}$, HELEN FERGUSON ${ }^{1}$, MATTHEW SCHWEICKLE ${ }^{1}$ \& STEWART VELLA ${ }^{1}$}

\section{${ }^{1}$ University of Wollongong, ${ }^{2}$ Australian Catholic University, ${ }^{3}$ University of New South Wales, ${ }^{4}$ Blackdog Institute, ${ }^{5}$ University of Canberra, ${ }^{6}$ University of Toronto, ${ }^{7}$ Nottingham Trent University \\ *Corresponding author: cswann@uow.edu.au @cswannpsych}

Sport is a motivating and engaging vehicle through which there is the potential for adolescents to acquire developmental assets. Resilience is one important developmental asset that underpins adaptive functioning over time (Sarkar \& Fletcher, 2014, Journal of Sports Sciences, 15, 1419-1434). The aim of this study was to develop, deliver, and evaluate a website and app-supported intervention to increase resilience and wellbeing in adolescent athletes. Based on formative research with adolescents, 'Your Path to Success in Sport' (YPTSS) was developed to consist of one face-to-face workshop of approximately one hour duration, and six modules of approximately $15 \mathrm{~min}$ in duration each delivered via website/mobile app. Content was based on sport-based resilience research (e.g., Galli \& Gonzalez, 2015, International Journal of Sport and Exercise Psychology, 3, 243-257), and informed by the Penn Resilience Program (e.g., Reivich et al., 2011, American Psychologist, 66, 25-34). The six modules addressed: problem-solving, controlling the controllables, managing thoughts, keeping your cool, playing to your strengths, and appreciating your team - all delivered through videos, scenarios, exercises, and reflective activities. YPTSS was delivered to adolescent male athletes through community sports clubs and sport academies. Following ethical approval for the study, and completion of the intervention, participants were given the opportunity to provide qualitative feedback about the program, to which 49 adolescent males (aged 12-17) responded. These data were analysed using deductive thematic analysis (Braun \& Clarke, 2006, Qualitative Research in Psychology, 3, 77-101), and processes including peer debrief were employed to enhance trustworthiness. Results are discussed in terms of: (i) the most helpful aspects of the program; (ii) impact of the program on their sport participation; (iii) perceptions of their resilience and wellbeing; (iv) suggestions for increasing usability and engagement; and (v) application of the skills/ content beyond sport. Generally participants reported the program to be effective, engaging, and transferable to other domains beyond sport (e.g., school). These results highlight the potential for larger-scale dissemination of the intervention, as well as providing feedback for its refinement moving forward. Future directions for rolling out YPTSS in youth sport will be discussed, with reflections on how best clubs, coaches/support staff, and organisations can engage adolescent athletes with the website and app.

D1.S3.3(6). I am great and I want to dominate: narcissism and performance under stress

\section{SHUGE ZHANG, ROSS ROBERTS, ANDREW COOKE \& TIM WOODMAN}

\section{Institute for Psychology of Elite Performance, Bangor University \\ *Corresponding author: pep61f@bangor.ac.uk}

Narcissists thrive in situations that provide them with an opportunity for personal glory (see Roberts, Woodman, Sedikides, 2017, International Review of Sport and Exercise Psychology, 1-24, for review). However, to date, the extant narcissism-performance literature has focused on narcissism at a global level, without recourse to consider the potentially differential effects of its so-called "adaptive" (e.g., selfassuredness) and "maladaptive" (e.g., dominance, entitlement seeking) components (Barry and Malkin, 2010, Journal of Research in Personality, 44, 684-690). Indeed, while adaptive narcissism might lead to good performance as a result of higher levels of confidence, the highest levels of performance might only occur when one is high in both adaptive and maladaptive narcissism, as the dominance and manipulation associated with maladaptive narcissism might drive individuals to exert more effort in the quest to achieve glory. Therefore, the aim of this study was to examine the hypothesized interaction between adaptive and maladaptive narcissism on performance. With institutional approval, fifty-eight golfers (handicap $=15.15 \pm 3.66$ ) completed the Narcissistic Personality Inventory (Raskin and Hall, 1979, Psychological Reports, 45, 590) and performed a putting task under practice, low and high stress. To create a highly stressful condition, our manipulations combined a variety of stressors including competition, rewards and punishment, glory and shame, pressurized instructions, negative feedback, and the use of video camera. We assessed anxiety using the Mental Readiness Form (Krane, 1994, The Sport Psychologist, 8, 189-202) and by assessing heart rate in each condition. Moderated regression analyses revealed significant interactions between adaptive and maladaptive narcissism on mean radial error $\left(\Delta R^{2}=.14, F\right.$ $=9.17, \beta=-.42, P=.004)$ under stress. Maladaptive narcissism was unrelated to error when adaptive narcissism was low $(\beta=.14, P=.693)$, but was associated with lower error, indicating better performance, when adaptive narcissism was high $(\beta=-.71, P=.080)$. These results were independent of participants' global narcissism scores and handicaps. The results indicate both adaptive and maladaptive components of narcissism are essential to achieve the highest performance under stress though pure maladaptive narcissism may be vulnerable under stress. Future research would do well to clarify and expand these effects and the underlying mechanism(s). 


\section{Day 1. Free Communications - Psychology (Session 2)}

\author{
D1.S3.4(1). Attentional focus and metacognitive \\ processes in recreational endurance runners: \\ exploring the impact of cognitive strategies on \\ longer-term activity adherence
}

NOEL BRICK ${ }^{1 *}$, MARK CAMPBELL ${ }^{2}$, RACHEL SHEEHAN ${ }^{2} \&$ TADHG MACINTYRE ${ }^{2}$

\author{
${ }^{1}$ Ulster University, ${ }^{2}$ University of Limerick \\ *Corresponding author: n.brick@ulster.ac.uk \\ @noelbrickie
}

Objective: The present study examined the attentional focus and metacognitive processes of recreational endurance runners. The emphasis was on understanding how attentional strategies are acquired, developed, and refined with continued participation in endurance activity. The impact of cognitive strategies on longer-term activity adherence was also of interest. Design: Semi-structured qualitative interviews were utilised. Method: following institutional ethical approval, ten beginner endurance runners (running on average $12.18 \pm 4.19$ months) were interviewed to explore retrospectively their attentional focus and cognitive strategy use during endurance running. Data were analysed using both deductive and inductive content analysis. Results: The runners reported utilising an array of attentional strategies and metacognitive processes to regulate cognition. These were not as diverse as those previously reported for elite runners, however. Cognitive strategies developed with experience and were often acquired as a consequence of effort-related sensory experiences. In addition, more experienced runners were also influential sources for cognitive strategy acquisition. Participating with other runners was also reported to reduce the self-regulatory demands of running. Conclusions: Acquiring and refining task-appropriate cognitive strategies, and developing metacognitive abilities may be important to maintain participation in endurance activity. The findings have implications for researchers and applied practitioners to understand how self-regulatory cognitions may contribute to longer-term endurance activity adherence.

\section{D1.S3.4(2). The psychological characteristics of developing excellence: academy coaches reflections on elite youth athletes, practical implications and actions two years on}

\section{MATTHEW B. CULLEN*, JONATHAN GLYNN, CONOR KILGALLEN, JAIME DIAZ \& CHARALAMPOS FOUNTOULAKIS}

\section{Aspire Academy \\ *Corresponding author: matthew.cullen@aspire.qa}

The purpose of this investigation was to explore the psychological attributes that facilitate the successful development of elite youth athletes. Promoting the behaviours accustomed with PCDES in developing athletes is an effective way to develop talent (Collins, Button, and Richards, 2011) In recognizing the role of psychological characteristics of developing excellence (PCDE's) in elite youth athletes the authors were able to highlight key psychological characteristics at certain developmental stages that support talent development. Data were collected from a sample of national level athletics coaches. The participants $(n=6)$ in this study, ranging in age from 35 to 65 years, were all employed as national youth coaches. A semi-structured interview was employed to investigate coaches perceptions of the PCDE evidenced in their most successful student athlete to graduate from the national sports academy. The interview guide approach (Patton, 2002) was employed because it specifies topics to be covered during the interview but does not necessarily specify the exact sequencing of the questions. Pilot interviews were conducted with an Olympic coach and a national team youth football coach. Data were analysed using a thematic narrative approach. Psychological factors were highlighted by the coaches as key determinants of those who emerged as talented and maintained excellence. Key psychological factors were identified consistently among coaches. The PCDEs appear similar to those employed in other performance domains and support increasing empirical evidence of the development of psycho-behavioural characteristics as a core feature of talent development processes. Practical implications, actions and future plans based on applied research are discussed.

D1.S3.4(3). Is every type of choice equal for youth rope skippers: the role of order and equally (un) attractive options in promoting task perception, engagement and intended perseverance

\section{GERT-JAN DE MUYNCK*, BART SOENENS, LOTTE DEGRAEUWE \& MAARTEN VANSTEENKISTE}

\section{Ghent University \\ *Corresponding author: gertjan.demuynck@ugent.be}

There is some debate among youth sport coaches with regard to the motivating effect of offering choice in training situations. Whereas some coaches align offering choice with a laissez-faire attitude, others see it as a useful motivating strategy. The current study used Self-Determination Theory (Deci \& Ryan, 2000, Psychological Inquiry, 11, 227-268) to examine whether providing youth rope skippers with choice is beneficial for their task perceptions, training engagement and intended perseverance. Specifically, we investigated whether various types of choice had a differential effect on the outcome variables. To do so, 120 youth rope skippers participated in an experimental field study for which institutional ethics approval was granted. They were randomly assigned to one out of three conditions. One condition offered participants a training session in which they were presented three times three exercises from which they could choose one to perform (i.e., option choice). The options 
within each choice were very similar in terms of challenge and perceived pleasure, with the first and last choice providing only creative exercises, while the second choice offered only repetitive and rather boring options. Participants in the other two experimental groups were either assigned to a training session that allowed selecting the order in which 3 exercises, chosen by the experimenter, were performed (i.e. action choice), or a training session in which both the exercises and their order were chosen by the experimenter (i.e. no choice). Upon completion of the training session, participants' task perceptions and training engagement were assessed in each condition. Results indicated that both option and action choice, compared to the no choice condition, enhanced autonomy need satisfaction $\left(\eta^{2}=.072^{*}\right)$ and diminished autonomy need frustration $\left(\eta^{2}=.084^{*}\right)$. However, transfer to greater engagement only occurred for the action choice condition $\left(\eta^{2}=.087^{*}\right)$. These results indicate that action choice is to be preferred above option choice to stimulate athlete engagement, although all effect sizes are rather small. One explanation of this finding might be that action choice is more structured, thereby facilitating the process of choosing, while option choice is too open and might elicit choice-stress. However, the lack of a beneficial effect of option choice in this experiment may also stem from the very low contrast between the options in this condition. Future research can examine this hypothesis by also including a condition that offers option choice with high contrast between the options.

D1.S3.4(4). The influence of music and music-video on self-selected exercise intensity and psychological response to treadmill exercise

\section{JASMIN HUTCHINSON* \& JESSICA BALLISTER}

\author{
Springfield College \\ *Corresponding author: \\ jhutchinson@springfieldcollege.edu
}

There is a large body of literature in support of the psychological and ergogenic benefits of listening to music during exercise. Much less is known about the combination of music and video, despite the ubiquity of this combination in exercise settings. Appropriately selected music has been shown to improve the affective experience of exercise and to enhance work output during self-paced aerobic exercise (see Karageorghis and Priest, 2012, International Review of Sport and Exercise Psychology, 5, 44-66, for review). Recent reports indicate that the affective benefits of music-video can exceed those of music under controlled exercise conditions (e.g. Hutchinson, Karageorghis \& Jones, 2015, Annals of Behavioral Medicine, 49, 199-211), but this effect has not yet been examined during self-paced exercise. Moreover, the possible ergogenic influence of music-video has not been investigated to date. Therefore, the aim of this study was to assess the influence of motivational music and musicvideo on self-selected exercise intensity and psychological response to exercise during treadmill walking. With institutional ethics approval, 21 aerobically trained female participants (mean age $=21.00 \pm 2.29$ years) completed three 15 min self-paced walking trials under three separate conditions; music, music-video, and control (silent). Trial order was fully counterbalanced across participants and all trials were conducted using a non-motorized treadmill. During each exercise bout, in-task dependent variables of perceived exertion (RPE), attentional focus, affective valence, and heart rate were measured at 5 min intervals. Total distance covered was recorded at the end of each trial. Participants covered a significantly $(P<.000)$, greater distance in the music-only condition $(1.59 \pm .19 \mathrm{~km})$ when compared to control $(1.50 \pm .18 \mathrm{~km}$; Cohen's $d=.49)$. Both music and music-video promoted significantly greater dissociation, more positive affect, and increased heart rate when compared to control (all $P<.001$ ). The results indicate that both music and music-video positively influence psychological responses during self-paced exercise. This holds important implications for exercise behaviour, as strategies that result in more positive (or less negative) affective responses to exercise are associated with better adherence to exercise programme and higher rates of overall physical activity. Music (but not music-video) had a positive ergogenic influence on self-selected exercise intensity relative to control, perhaps indicating a different mechanism of action for psychological and ergogenic effects of music and music-video during self-paced exercise.

\section{D1.S3.4(5). Music hath charms to soothe the} savage beast but what of the savage workout? Psychological and psychophysiological effects of recuperative music

\section{COSTAS I. KARAGEORGHIS*1, ANDREW C. BRUCE ${ }^{1}$, SUZANNE T. POTTRATZ ${ }^{2}$, REBECCA C. STEVENS ${ }^{1}$, MARCELO BIGLIASSI ${ }^{1}$ \& MARK HAMER ${ }^{3}$

\author{
${ }^{1}$ Brunel University London, ${ }^{2}$ East Central University, \\ ${ }^{3}$ Loughborough University \\ *Corresponding author: \\ costas.karageorghis@brunel.ac.uk \\ @SAVIBrunel
}

Recent years have seen increased research interest in the application of music as a means by which to expedite and enhance post-exercise recovery. Researchers have used entrainment theory (Thaut, 2008, Rhythm, music and the brain: Scientific foundations and clinical applications. New York: Routledge) as a conceptual framework. The theory details how bodily pulses such as brainwaves, heart rate and respiration rate can be up-/down-regulated by a musical stimulus. There is a distinction in the literature between recuperative music, which is used at the end of an exercise session and respite music, which is used in between highintensity intervals (Jones, Tiller, \& Karageorghis, 2017, Physiology \& Behavior, 170, 106-114). Studies examining recuperative music have delivered promising initial results in relation to its psychological and psychophysiological effects, albeit that most have been blighted by methodological weaknesses (e.g., providing little justification for the choice of musical stimuli). The purpose of the present study was to examine the effects of three conditions (slow, sedative music, fast, stimulative music, and a no-music control) on psychological and psychophysiological recovery processes following exhaustive cycle ergometer exercise. With institutional ethics approval, core affect, salivary cortisol, 
heart rate $(H R)$, and blood pressure $(B P)$ were measured before exercise, immediately after, and in 10-, 20- and 30min intervals during static recovery ( 21 women and 21 men; mean $+s$ age: $20.9+1.7$ years). Mixed-model 3 (condition) $x$ 4 (time) $\times 2$ (gender) ANCOVAs were used to analyse cortisol, HR, systolic blood pressure, and diastolic blood pressure. Affective valence and arousal were analysed using a 3 (condition) $\times 4$ (time) $\times 2$ (gender) MANOVA. Focusing only on statistically significant findings, the largest decline in affective arousal between active and passive recovery phases was evident in the slow, sedative condition $\left(\eta_{\mathrm{p}}{ }^{2}=\right.$ .50). Interestingly, women had a more pronounced reduction in arousal than men in the slow, sedative music condition $\left(\eta_{p}{ }^{2}=.09\right)$. The same condition was most effective in returning participants' HR towards resting levels $\left(\eta_{\mathrm{p}}{ }^{2}=.06\right)$ and salivary cortisol levels also tended to be lower $\left(n_{\mathrm{p}}{ }^{2}=\right.$ .11). There was a main effect of condition for affective valence indicating that the slow, sedative condition elicited more positive affective responses than the other two conditions $\left(n_{\mathrm{p}}{ }^{2}=.12\right)$. Collectively, the present findings add some weight to the notion that slow, sedative music can expedite recovery processes. Future research might focus on additional recovery indices, such as HR variability and electroencephalography.

\section{D1.S3.4(6). Community level rugby union players' concussion experiences}

PAUL SELLARS ${ }^{1 *}$, STEPHEN MELLALIEU ${ }^{1} \&$ SHARIEF HENDRICKS $^{2}$

${ }^{1}$ Cardiff Metropolitan University, ${ }^{2}$ University of Cape Town

*Corresponding author: psellars@cardiffmet.ac.uk @p_sellars

Rugby union is as a sport with a high potential risk of injury from concussion, with associated short and long term negative health outcomes. The community level (i.e., semi-professional, amateur, and recreational) of the game at which the sport is played is where the greatest risks and dangers occur, due to the largest participation numbers and lowest level of provision of medical support and care. The current study sought to explore players' concussion experiences, specifically in relation to the experience and management of the injury. The study used interpretative phenomenological analysis (IPA), which involved the researcher endeavouring to understand and explain participants' perspectives, with an overarching aim of making sense of participants' worlds. Following institutional ethics approval, data collection was conducted via semi-structured interviews with 11 purposively sampled participants aged between 20 and 35 (mean age: $27 \pm 4.4$ years), from the UK $(n=10)$ and New Zealand $(n=1)$, who were currently participating in, or had recently retired from community level rugby union. Following interview transcription, each case was individually analysed using IPA stages. Next, the 11 individual cases were compared, similar themes were combined, and a master table of participants' themes was produced. Combined, themes related to: level of concussion knowledge and understanding; the nature of the concussion incident; factors influencing the removal from field of play; acute and chronic post incident symptoms; symptom management for return to play; and, concerns over the management of concussion incidents. Experiences from players highlighted the lack of medical care and attention players receive at community level during matches and return to play process; the role of social and personal factors in influencing players' appraisals of whether to be removed or remove themselves from field of play; and, the subsequent influences which the injury can have upon physical and psychological wellbeing. The findings suggest the need, at the community level of the sport, for further education and training around the management of concussion incidents and the support (informational and social) provided throughout the return to play process. In order to promote effective concussion management, future education and training may benefit from incorporating specific factors relating to a club's performance level, player numbers, team culture, and the resources available. 


\section{Day 1. Free Communications - Sport and Performance}

\author{
D1.S3.5(1). Which measures of training load \\ explains the change in neuromuscular strength \\ post-game in soccer players?
}

IBRAHIM AKUBAT ${ }^{1 *}$, CHRIS WILCOX ${ }^{2}$, LEE TAYLOR ${ }^{3,4}$ STEVE BARRETT ${ }^{5}$, DANIEL PEART ${ }^{6}$, CHRIS TOWLSON ${ }^{2}$, JAMES BRAY ${ }^{2}$, MANUEL LAPUENTE SAGARRA ${ }^{7,8}$, TONY MYERS $^{1} \&$ GRANT $A B T^{2}$

\author{
${ }^{1}$ Newman University, ${ }^{2}$ University of Hull, ${ }^{3}$ Aspetar, \\ ${ }^{4}$ University of Loughborough, ${ }^{5} \mathrm{Hull}$ City $F C$, \\ ${ }^{6}$ Northumbria University, ${ }^{7}$ INEFC Lleida, ${ }^{8}$ Technifutbol \\ *Corresponding author: \\ ibrahim.akubat@newman.ac.uk \\ @trainingimpulse
}

The neuromuscular recovery from soccer match-play has been reported to take $48-72 \mathrm{~h}$ with inter-individual variability. This could be partly explained by the different extents to which players exert themselves. Measurement of training and competition load has increased in recent years with the advent of MEMS technology and heart rate monitors. A number of external and internal training load measures are now used and have been shown to relate to changes in fitness. However the relationships with acute recovery are less well known. Therefore the purpose of this study was to assess the relationships between different measures of training load and the changes in neuromuscular performance measures. Following institutional ethical approval, thirteen university level soccer players took part in a soccer simula-

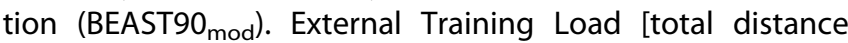
(TD), high intensity distance above $15 \mathrm{~km} \cdot \mathrm{h}^{-1}$ (HID), mean metabolic power (MMP), distance covered at high metabolic power above $20 \mathrm{w} \cdot \mathrm{kg}^{-1}(\mathrm{HP})$ \& PlayerLoad $\left.{ }^{\mathrm{Tm}}(\mathrm{PL})\right]$, was collected using MEMS devices $(5 \mathrm{~Hz}$ GPS, $100 \mathrm{~Hz}$ Accelerometer). Data from a lactate threshold test was used to calculate objective internal TL (iTRIMP) from HR recorded during the simulation. Subjective internal TL (session RPE) was also measured post game. An isokinetic dynamometer was used to determine concentric hamstring $(\mathrm{CH})$, concentric quadriceps $(\mathrm{CQ})$, eccentric hamstring $(\mathrm{EH})$ and eccentric quadriceps $(\mathrm{EQ})$ strength pre-game $(\mathrm{T} 1)$, postgame (T2), $24 \mathrm{~h}$ post-game (T3) and $48 \mathrm{~h}$ hours post-game (T4) game through a range of $90^{\circ}$ at an angular velocity of $120 \mathrm{os}^{-1}$. Linear regression analysis was used to determine which training load measures explained the variance in relative changes in neuromuscular strength. Relationships where confidence intervals did not cross 0 where found with iTRIMP \& CH $\left(r^{2}=0.58\right)$, TD \& CQ $\left(r^{2}=0.41\right), H I D \& C Q\left(r^{2}\right.$ $=0.48), \mathrm{PL} \& \mathrm{CQ}\left(r^{2}=0.54\right)$ and $\mathrm{PL} \& \mathrm{CQ}\left(r^{2}=0.60\right)$ at T2. TD \& EQ $\left(r^{2}=0.35\right)$, HID \& CQ $\left(r^{2}=0.41\right), H I D \& E H\left(r^{2}=0.54\right) \&$ PL \& EQ $\left(r^{2}=0.70\right)$ T3. SRPE \& CH $\left(r^{2}=0.65\right)$ and MMP \& CQ $\left(r^{2}=0.70\right)$ at T4. Several training load measure may explain the changes in neuromuscular function at different timepoints during the recovery period. Practitioners may find the relationship between HID \& EH for the reduction of hamstring injury risk of particular interest. This study is the first to examine the relationships between a comprehensive range of training load measures and changes in neuromuscular strength.

\section{D1.S3.5(2). Effects of brain endurance training on endurance exercise performance}

\section{NEIL DALLAWAY, SAM LUCAS \& CHRISTOPHER RING}

\section{University of Birmingham \\ *Corresponding author: nxd898@bham.ac.uk @npdall}

Mental fatigue (MF) impairs endurance exercise performance (Cutsem et al., 2017, Sports Med. doi:10.1007/s40279-0160672-0). Brain endurance training (BET) - such as engaging in cognitive tasks during exercise - can develop resilience to MF and improve physical performance compared to physical training alone (Marcora, Staiano, \& Merlini, 2015, ACSM annual meeting. San Diego). Only one study to date has demonstrated the effectiveness of BET and the underlying mechanisms have yet to be determined. The aims of this study were to investigate, if BET enhances performance over physical training, and potential mechanisms. With institutional ethics approval, participants completed pre- and post-testing sessions either side of a training programme. Pre-training: 36 participants completed a rhythmic handgrip task requiring generation of as much force as possible once a second for $300 \mathrm{~s}$, performed under 3 counterbalanced conditions: following $600 \mathrm{~s}$ of a 2-back memory/attention task (subsequent); while performing a 2-back task (concurrent); and on its own (solo). Cardiac activity (ECG), electromyographic (EMG) forearm activity, pre-frontal cerebral haemodynamic (near infrared spectroscopy), and force were recorded. Training: Participants (randomized to a Control or BET group) completed 24 ( 6 weeks) submaximal hand contractions sessions. The BET group also completed concurrent cognitive tasks (2-back, word incongruence Stroop). Post-training: Repetition of the pre-training protocol. Measures of motivation, physical and mental exertion, mental fatigue and mood were collected via self-report. Endurance performance, across the 3 tasks, improved more following BET (23\%) than Control (5\%) $(\lambda=0.737, F(1,34)=$ $\left.12.148, P=.001, \eta^{2}=.263\right)$. Increased performance in Controls was associated with reduced pre-frontal cortex oxygenation over time relative to pre-training and post-training BET group responses $(P<.05)$. Heart rate variability (SDNN) increased more in the BET group, over time, post training $(P<.05)$. Interest and enjoyment of the tasks declined more in the BET group following training $(P=.01)$. We have demonstrated that six weeks of brain endurance training improved physical performance more than physical training alone and was achieved for the same heart rate, muscle activity, motivation and RPE as the Control group. However, the performance increase in the BET group occurred without a decrease in pre-frontal oxygenation as was seen in control group. The increase in heart rate 
variability in the BET group post training, relative to Controls, indicates a reduction in sympathetic nervous system activity and suggests less effort for the physical tasks. The reduction in interest and enjoyment for BET relative to Control, post training, suggests a need for more challenging and exciting tasks.

D1.S3.5(3). Pacing and performance in head-tohead competition: external cues as invitations for actions

\section{MARCO KONINGS* \& FLORENTINA HETTINGA}

\section{University of Essex \\ *Corresponding author: mkonings@essex.ac.uk @MJ_Konings}

Exercisers are required to continuously decide how and when to invest their available energy resources, a process also known as pacing (Smits, Pepping \& Hettinga, 2014, Sports Medicine, 44, 763-775). Athlete-environment interactions are crucial in this pacing decision-making process, and multiple external invitations for action are simultaneously and continuously presented to an exerciser in real-life competitive situations (Hettinga, Konings, \& Pepping, 2017). The present study aimed to identify which external cues invite elite short-track speed skaters to modify their pacing behaviour. With institutional ethics approval, lap times of elite 500, 1000 and $1500 \mathrm{~m}$ short-track speed skating competitions between 2011-2016 ( $n=34095$ races) were collected. Log-transformed lap and finishing times were analysed with mixed linear models. Separate analyses were performed for each event. The fixed effects in the model were Sex, Season, Stage of competition, Start position, Competition importance, Event number per tournament, Number of competitors per race, Altitude, and Time qualification (i.e. the opportunity to qualify for the next stage as one of the time fastest skaters who did not qualify via finishing position). The random effects of the model were Athlete identity (between-athletes differences) and the residual (within-athlete race-to-race variation). Precision of the estimates of CV are shown as $95 \%$ confidence limits. A spreadsheet was used to combine and compare fixed effects and CVs (Hopkins, 2006, A Spreadsheet for Combining Outcomes from Several Subject Groups. Retrieved from http://www.sportsci.org/ 2006/wghcom.htm). Several external cues appeared to alter the pacing decisions of elite skaters for the 1000 and $1500 \mathrm{~m}$ event, such as the number of competitors in a race (a higher number of competitors evokes most likely a faster initial pace; $C V=1.9-9.3 \%$ ), the stage of competition (likely to most likely, a slower initial pace is demonstrated in finals; $\mathrm{CV}=-1.4-2.0 \%$ ), the possibility of time qualification (most likely faster initial pace; $\mathrm{CV}=2.6-5.0 \%$ ) and competition importance (most likely faster races at the Olympics; $\mathrm{CV}=1.3-3.5 \%)$. For the $500 \mathrm{~m}$ event, stage of competition and start position affected the displayed pacing behaviour. In a setting of high ecological validity, the present findings demonstrate several external cues are able to evoke a response in pacing behavior. This emphasizes the importance of the relationship between perception and action regulation, especially during competition. To understand the decision-making process and tactics involved in pacing, both the internal state of the exerciser as well as the external world presented towards the exerciser need to be considered.

\section{D1.S3.5(4). Exploring the barriers and enablers to improving organizational functioning within Olympic sport programmes}

\section{CONOR MOLAN ${ }^{1 *}$, SEAMUS KELLY ${ }^{1}$, RACHEL ARNOLD ${ }^{2}$ \& JAMES MATTHEWS ${ }^{1}$ \\ ${ }^{1}$ University College Dublin, ${ }^{2}$ University of Bath \\ *Corresponding author: conor.molan@ucdconnect.ie @ConorMolan}

The Olympic sport programmes of national sport organizations (NSO) must continuously evolve to avoid falling behind competitors and the increasing standards of professionalization and performance expected within elite sport environments. This drive to continually enhance performance requires improvement to how the programme is functioning. However, due to the complex structures and layers within NSOs, the implementation of such change within Olympic sport programmes. Despite calls for research in this area, to date it remains an underexplored phenomenon (Wagstaff, Gilmore, \& Thelwell, 2016, Journal of Change Management, 16, 38-54). The purpose of this study was to explore the barriers and enablers to improving organizational functioning within Olympic sport programmes. The study adopted a critical realist position, using qualitative methods to interpret the experiences of individuals who have experienced this phenomenon. Following institutional ethical approval, purposive sampling was used to identify individuals who had worked with Olympic Sport programmes during periods of organizational change (e.g. chief executive officers [CEO], performance directors, management consultants, lead psychologists). In total, 14 semi-structured participant interviews were conducted, which ranged from 41 to $67 \mathrm{~min}$ in duration $(M=52.1, S D=8.1)$. Thematic analysis was used to identify and group units of meaning related to key barriers and enablers to improving organizational functioning. A four-phase process was undertaken to enhance rigor and trustworthiness. Barrier-related themes emerged at four levels: individual-level (e.g. reluctance for volunteers to lose control), inter-personal level (e.g. misaligned relationship between CEO and performance director), intra-organizational level (e.g. board lack understanding of elite-level sport), and sport system level (e.g. membership resist further investment in elite-level sport). Similarly, enablerrelated themes were identified at individual level (e.g. clear understanding of role and responsibilities), inter-personal level (e.g. check and challenge staff performance), intra-organizational level (e.g. legitimizing need for change at board-level), and sport system level (e.g. communicate international benchmarks for the sport). In terms of theoretical and practical contributions, the findings suggest that applying a socio-ecological perspective may provide change agents (such as sport leaders, psychologists, and consultants) with a clearer understanding of the barriers/enablers improving organizational functioning and the levels at which they occur. This approach can inform better 
development, delivery and evaluation of a future change programme, therefore, potentially increasing the likelihood of its success.

\section{D1.S3.5(5). The anaerobic power reserve and its predictive ability of short duration maximal power output in professional road cyclists}

\section{DAJO SANDERS ${ }^{1 *} \&$ MATHIEU HEIJBOER ${ }^{2}$}

\author{
${ }^{1}$ Newman University, ${ }^{2}$ Lotto-NL Jumbo Professional \\ Cycling Team \\ *Corresponding author: dajosanders@gmail.com \\ @dajosanders
}

In cycling, the anaerobic power reserve (APR) is defined as the difference between maximal sprint peak power output and power output at $\mathrm{VO}_{2 \max }$. Studies have used the APR range to set out the minimal and maximal values of a high-power duration curve. Subsequently, an exponential decay model is used to describe the decrement in power over time and predict power output over of all-outs efforts lasting from a few seconds to a few minutes. Previous studies have shown promising results in applying this model to cycling, however, there is still limited evidence of the applicability of the APR model in elite cyclists. Therefore, the present study examined if short-duration (5-180 s) highpower performances achieved during competitions can be predicted using the APR model in professional road cyclists. In addition, it will be evaluated if modifications to the previously established exponential constant describing the decrement in power output over time could improve overall predictive ability of the model. With institutional ethics approval, 12 professional cyclists from a World Tour cycling team participated in this investigation. Using the maximal aerobic and sprint peak power output assessed in the field and an exponential constant describing the decrement in power over time, a power-duration relationship was established for each participant. Mean maximal peak power outputs of different durations were collected from competition data and compared to the predicted power output by the APR model. An iterative best-fit approach was used to determine a modified exponential constant for this cohort. Using the originally proposed exponential constant $(k=0.026)$ the APR model predicted the short-duration performances within an average of $42 \mathrm{~W}$ and $6.0 \%$. The modified constant $(\mathrm{k}=0.02921)$ predicted performance within a mean of $40 \mathrm{~W}$ and $3.9 \%$. This study shows that in professional cyclists, high-power performance achieved during competitions can be predicted using the APR model. The decrement in power output during all-out performances of short duration (5-180 s) fits well with a general relationship using a single exponential decay model. The determination of individual power duration curves can contribute to the individualisation of training intensity and demand during high-intensity interval training and may help coaches set the correct intensities for different work to rest ratios to achieve the desired adaptation for cyclists with differing competition goals. In addition, modified exponential constants using a best-fit approach can contribute to improving overall predictability of the APR model in different sports.

\section{D1.S3.5(6). The effects of post-activation potentiation on Counter Movement Jump in developmental swimming athletes}

\section{KIRSTEN GRANT*, MARK MURRAY \& JED MCKERNIE}

\section{University of the Highlands and Islands *Corresponding author: kirsten.grant.ic@uhi.ac.uk}

Post-activation potentiation (PAP) is an excited or sensitive neuromuscular condition following intense loading (Hodgson, Docherty \& Robbins, 2005, Sports Medicine, 35, 585-595). Jeffreys (Jeffreys, 2008, Professional Strength and Conditioning, 12,17-25) reviews a number of PAP studies and considers the application of PAP to sports performance and concludes that the optimal application of PAP will likely be highly individual, and requires further research. PAP can provide coaches with a new tool to potentially impact sports and exercise performance. The aim of the study was to identify the optimal timeframe to initiate the post-activation potentiation effect on jump ability for developmental swimming athletes in order to inform warm up protocols. This study investigated the effects of post-activation potentiation on Counter Movement Jump in developmental swimming athletes. With institutional ethics approval, 11 Regional Age Grade Swimmers ( $n=11, M: F$ 5:6), age-range from 12-16 years and strength training history of 6 months completed a barbell warm up of key exercises, followed by 3 sets of 5 reps @ 85\% of 1 Rep Max, Back Squat (based on their training diaries). Baseline measurements of Counter Movement Jump (CMJ) using Optojump photocell system and CMJ protocols were measured. Immediately after the Back Squat, the participants performed the CMJ after rest periods of 30s, 4 mins, 8 mins, 12 mins and 16 mins on 5 consecutive weeks. The mean CMJ scores were calculated. A decrease in performance after $30 \mathrm{~s}, 4$ mins, 12 mins and 16 min rest was observed. There was a slight performance improvement after 8 mins rest. A paired-sample $t$-test was used to determine whether there was a significant difference in jump height following 8 min rest after $3 \times 5$ back squats but there was no evidence to suggest that participants performed statistically significant greater jump heights ( $t=$ $0.567, P=0.583)$. Limitations of the study include the training history of athletes - previous studies indicate that stronger subjects will see greater PAP effects. Future research should further investigate the optimal rest time following heavy resistance training to elicit the PAP effect to inform practical application of warm-up protocols. 


\section{Day 1. Posters - Biomechanics and Motor Behaviour}

\author{
D1.P1. Multi-segment synergies during the tennis \\ serve in expert performers
}

\section{ANDREW BARNES*, STUART BONNER, JONATHAN FRASER \& MOHSEN SHAFIZADEH}

\section{Sheffield Hallam University \\ *Corresponding author: a.barnes@shu.ac.uk}

Exploiting joint variability is one of the main principles of motor control theory and has been used to explain mechanisms related to expert performance (Lee, 1984, Journal of Motor Behaviour, 16, 135-170). The tennis serve is a complex motor skill that requires synchronisation among active body parts for an effective utilisation of the kinetic chain to maximise racket head velocity (Kibler, 2009, Medicine and Science in Tennis, 14, 5-8). Therefore, the aim of this study was to explore the intersegment synergies in upper body movements during the service action. Following institutional ethics approval, nine expert male and one expert female tennis players (British Lawn Tennis Association rating system 1.1-5.2; age: $34.1 \pm 5.3$ years; mean mass: $80.3 \pm 14.3 \mathrm{~kg}$; mean stature: $178.5 \pm 8.9 \mathrm{~cm}$ ) were recruited, the majority of which were right handed (70\%). Participants completed ten serves landing within the service box against an opposition player on a full sized tennis court while kinematic data were collected at $100 \mathrm{~Hz}$ using a wireless 3D motion analysis capture system (STT Co, Spain). Sensors defined a seven segment model (right and left hand, right and left lower arm, right and left upper arm and head) with joint angles calculated as the distal segment relative to the proximal segment and normalised to a static standing trial. All time series were interpolated to the length of the service action and analysed using principle component analysis (PCA) to reduce the complex skill into key subsets of technique. Results of the principal component analysis showed that this biomechanical model extracted a large proportion of the tennis serve (total variance: $90.55 \pm 2.27 \%$ ). There were 4 extracted principal components ( $P C s$ ) from 17 joint motions. The $P C s$ identified were ball toss set-up $\left(P_{1}=40.18 \pm\right.$ $9.92 \%)$, forward kinematics chain $\left(\mathrm{PC}_{2}=26.73 \pm 4.51 \%\right)$, counter-movement loading ( $\left.\mathrm{PC}_{3}=15.74 \pm 6.89 \%\right)$ and contralateral acceleration-deceleration $\left(\mathrm{PC}_{4}=5.89 \%\right)$. More specifically, the racket arm defined $50.37 \%$ of total variance from 3 PCs. The current findings demonstrated that expert performers reduce the joints dimensionality through creating functional motor synergies in different phases of the serve. The serve is a multi-segment motor skill that relies on a complex coordination between racket arm, non-racket arm and head movements. These findings may be of use when designing specific coaching strategies aimed at developing the coordination between multiple segments during the service action.
D1.P2. Comparing visual search strategies in successful and unsuccessful one-on-one defensive situations in soccer

\section{BECKY CONWAY ${ }^{1,2}$ \& ADAM HAWKEY ${ }^{1,3^{*}}$ \\ ${ }^{1}$ Southampton Solent University, ${ }^{2}$ Southampton Football Club, ${ }^{3}$ University of Dundee \\ *Corresponding author: adam.hawkey@solent.ac.uk @a_hawkey}

While the majority of research investigating perceptual skills within sports performance has focused on comparisons between expert and novice performers (Memmert et al., 2009, Psychology of Sport and Exercise, 10, 146-151; Allen et al., 2006, The Quarterly Journal of Experimental Psychology, 59(6), 1101-1116), evidence is required to support whether these differences exist when comparing successful and unsuccessful patterns of play. These observational strategies can be investigated through an individual's gaze behaviour within a simulated environment, with the number of fixations quantifying gaze control and attention (Lex et al., 2015, PLOS ONE, 10(2), e0118219). The purpose of the current study was to compare differences in visual search strategies between unsuccessful and successful one-on-one defensive situations. Following institutional ethics approval, three $(n=3)$ female junior soccer players (mean: age $=14$; mass $=$ $51.6 \pm 5.9 \mathrm{~kg}$; height $=1.66 \pm 0.06 \mathrm{~m}$ ) volunteered for the study. All participants were part of a regional talent club of a local English Premier League team; one was a skilled defender, while the other two were skilled attacking players. A oneon-one zone consisting of an attacker-defender dyad was utilised to analyse visual search strategies in successful and unsuccessful tackles. A total of twelve trials were completed consisting of seven successful tackles and five unsuccessful tackles. Visual search strategies of the defender were recorded using a head mounted gaze tracking device (Tobii Pro Glasses Eye Tracker 2, Tobii Technology). Three measures of search rate were examined: mean number of fixation locations per trial, mean number of fixations per trial, and the mean duration of fixation per trial. There were significant differences between trials in the mean number of fixations ( $t$ $(10)=-4.79, P=0.001)$, and the mean fixation duration, $(t(10)$ $=12.01, P<0.001)$. The search rate of successful tackles was characterised by more fixations of a shorter period, in more informative areas of display, compared to the unsuccessful tackles. Results of the current study suggest that an increase in focus on an opponent's postural orientation leads to a subconscious ability to activate scene schemas, thus enhancing detection and recollection of object functionality. Subsequently, this appears to lead to superior decision-making, thus successful motor responses. Defenders should therefore be encouraged to alternate their gaze away from focusing on the ball and place a greater focus on pinpointing their vision at the hips of the opponent. 
D1.P3. Investigating the effects of vibration on mechanical efficiency during cycling

\section{ADAM HAWKEY ${ }^{1,2^{*}}$, PIERRE HOCKEY ${ }^{3}$, KIERAN BEDFORD $^{3} \&$ DANIEL ROBBINS ${ }^{3}$}

\section{${ }^{1}$ Southampton Solent University, ${ }^{2}$ University of Dundee, \\ ${ }^{3}$ University of Bedfordshire \\ *Corresponding author: adam.hawkey@solent.ac.uk @a_hawkey}

Mechanical efficiency (ME), defined as external work accomplished $\div$ energy input x 100 (McArdle et al., 2006, Essentials of Exercise Physiology), is regularly used by exercise professionals to ascertain how much energy is used in relation to the minimal amount of work required to complete a task or movement (McBride et al., 2015, Journal of Sports Sciences, 33(13), 1388-1395). While ME has been extensively investigated in a cycling context (Jobson et al., 2012, Journal of Science and Cycling, 1(1), 3-8) the specific effect of vibration on ME during cycling has yet to be investigated. Therefore, the purpose of the current study was to determine if vibration had any influence on ME during cycling. Following institutional ethics approval, 20 undergraduate students were recruited using the convenience sampling method. Following a standardised, incremental, 5-min warm-up, all participants cycled for $15 \mathrm{~min}$ on a stationary cycle ergometer in both vibration and non-vibration conditions: both conditions were standardised at a rate of $80 \mathrm{~W}$ (equating to 70RPM and 65RPM in the non-vibration and vibration conditions respectively). During each condition, oxygen consumption was collected and measured using a Cortex Meta Control 3000, from which the gross mechanical efficiency (GE: a simpler variant of ME without correction for restful oxygen intake) was calculated. To minimise the effects of fatigue, the two conditions were separated by $24 \mathrm{hrs}$. As the vibration data was not normally distributed, a Wilcoxon signed rank test was used to analyse the data. Results reported a significant increase $(P<0.001)$ in oxygen consumption during the vibration condition $(24.5 \pm 3.8 \mathrm{ml} . \mathrm{kg}$. $\left.\mathrm{min}^{-1}\right)$ compared to the non-vibration condition $(16.9 \pm 2.7$ $\left.\mathrm{ml} . \mathrm{kg} \cdot \mathrm{min}^{-1}\right)$. Subsequently, there was also a significant reduction $(P<0.001)$ in $\mathrm{GE}$ during the vibration condition $(15.77 \pm 2.8 \%)$ compared to the non-vibration condition $(23.1 \pm 3.5 \%)$. These findings suggest that being exposed to vibration during cycling has the potential to significantly increase energy demand, and negatively affect an individual's efficiency. This has implications for the cyclist as increased oxygen consumption without increased cadence or resistance in terms of cycling training will negatively affect performance. It is, therefore, recommended that cyclists utilise vibration exposure in a training environment for the potential benefit of increasing their tolerance to the vibration they may encounter during competition. Further investigation is now required to ascertain how vibration could affect muscle activation during cycling and evaluate methods designed to dampen such exposure.

\section{D1.P4. Effects of backpack load on dual-task postural stability in children}

\section{MATHEW HILL*, RUTH BOAT, JOSH HURST, LEANNE RAYMOND \& MICHAEL DUNCAN}

\section{Coventry University \\ *Corresponding author: matt.hill@coventry.ac.uk @DrMathewHill}

Carrying a backpack contributes to reduced postural stability during standing in children (Pau et al., 2011, Gait \& Posture, $33,195-199)$. Standing balance is also affected by cognitive interference tasks (Boonyong et al., 2012. Gait \& Posture, 35, 428-434). Considering children perform daily activities while concomitantly carrying school bags and performing attention-demanding tasks, examining the effects of external loading in dual task situations has clear relevance. The aim of this study was to investigate the effects of backpack carriage and/or divided attention on standing balance in children. Following institutional ethics approval, nine children (age; $7.9 \pm 1.3$ years, mass; $27.2 \pm 7.9 \mathrm{~kg}$, height $128.4 \pm$ $9.6 \mathrm{~cm}$ ) performed quiet standing balance tests under single(ST) and dual-task (DT) situations in unloaded (no backpack [0\%]) and loaded (backpack with 20\% and 30\% of body mass) conditions. Participants stood on a force plate, with the feet placed on two $30^{\circ}$ oriented foot shapes, to ensure a common reference position. The centre of pressure (COP) displacement in the anteroposterior $\left(\mathrm{COP}_{\mathrm{AP}}\right)$ and mediolateral $\left(\mathrm{COP}_{\mathrm{ML}}\right)$ directions, and mean $\mathrm{COP}$ velocity $\left(\mathrm{COP}_{\mathrm{V}}\right)$ were calculated during $30 \mathrm{~s}$ quiet standing. The cognitive interference task comprised of serial subtractions by three, starting from random numbers between 50 and 100. Postural sway outcomes were analysed by separate two-way repeated measures ANOVA (load; 0\%, 20\% and 30\% $\times$ attention; ST, DT). Results showed main effects of load and attention for all evaluated sway measures (all $P<$ 0.05). Specifically, additional backpack load did not alter postural sway during ST conditions (all $P>0.05$ ). However, additional attentional-demand affected postural sway measures during all load conditions (all $P<0.05$ ). The increase in sway from ST to DT systematically increased with greater external loading $(0 \% ; d=1.3-1.4,20 \% ; d=$ $1.7-1.8,30 \% ; d=2.0-2.5)$. There were no differences between cognitive task performance during seated and standing conditions $(P>0.05)$. The present study indicates that additional cognitive, but not physical load demand influences movement velocity and directional control during quiet standing in children. Specifically, children are able to effectively compensate additional demands that a heavy backpack poses on postural stability. However, they were less able to maintain standing balance in cognitively challenging DT situations. The results illustrate a reduction in postural stability during the concurrent performance of attention-demanding tasks that are probably due to the inability of children to allocate attention properly between standing and a cognitive-motor interference task. 


\section{Day 1. Posters - Physical Activity for Health}

D1.P5. Influence of fed vs. overnight-fasted state exercise on whole-body and skeletal muscle substrate utilisation in obese or overweight/ centrally obese men

NURUL-FADHILAH ABDULLAH ${ }^{1,2^{*}}$, HELEN E. BRADLEY ${ }^{1}$, SCOTT R. ROBINSON ${ }^{1}$, KONSTANTINOS N. MANOLOPOULOS $^{3}$ \& GARETH A. WALLIS ${ }^{1}$

\section{${ }^{1}$ School of Sport, Exercise and Rehabilitation Sciences, College of Life \& Environmental Sciences University of Birmingham, ${ }^{2}$ Department of Health Sciences, Faculty of Sport Sciences and Coaching, Universiti Pendidikan Sultan Idris, ${ }^{3}$ Institute of Metabolism \& Systems Research, College of Medical \& Dental Sciences, University of Birmingham \\ *Corresponding author: nfa425@bham.ac.uk}

An elevated storage of fat within muscle (intramuscular triglyceride; IMTG) is common in obese individuals and is associated with increased risk of insulin resistance. Exercise performed in overnight-fasted state has been shown to augment IMTG turnover in lean individuals, and this may reduce the accretion of insulin-resistance inducing lipid metabolites. However, it has not yet been studied in obese populations. We aimed to investigate the effects of a single exercise bout performed either before or after breakfast on exercise substrate and IMTG utilisation in obese or overweight/centrally obese men. Using a randomized, crossover design, twelve obese or overweight/centrally obese (BMI $30.2 \pm 3.5 \mathrm{~kg} / \mathrm{m}^{2}$; waist circumference $105.7 \pm 11.6 \mathrm{~cm}$ ) but otherwise healthy young $(29.7 \pm 9.9$ years) men performed 1-h of constant load bicycle exercise (65\% VंO2max) followed by 3-h of controlled recovery. On one occasion, exercise was performed after an overnight fast (Fasted) and a standardised breakfast ( $25 \%$ daily energy intake; $65 \%$ carbohydrate, $20 \%$ fat and $15 \%$ protein) was consumed immediately after exercise, and on the other they consumed $90 \mathrm{~min}$ before exercise (Fed). Skeletal muscle biopsies were collected immediately before and after exercise for determination of IMTG content and muscle glycogen concentration $(n=8)$. During exercise, fat oxidation $(0.31 \pm 0.09$ vs. $0.22 \pm 0.07 \mathrm{~g} /$ min respectively, $P<0.05$ ) and concentration of plasma glycerol $(P<0.001)$ and non-esterified fatty acids $(P<$ 0.001 ) were significantly higher in Fasted vs. Fed. Muscle glycogen use during exercise was similar in both trials ( 60\% reduction, $P<0.001)$. There was a significant diettime interaction for Type I fibre IMTG content $(P<0.05)$. Type I fibre IMTG content pre-exercise was higher in Fasted vs. Fed but not statistically significant $(13.78 \pm 4.06$ vs. $9.78 \pm$ $4.81 \%)$. Exercise performed in fasted state significantly reduced Type I fibre IMTG content $(13.8 \pm 4.1$ to $10.3 \pm 3.4$ $\%, P<0.01)$, but no significant change was seen in the fed state $(9.8 \pm 4.8$ to $11.2 \pm 4.9 \%, P=0.362)$. No significant difference between trials existed for post-exercise Type I fibre IMTG content. This study shows that in obese or overweight/centrally obese men, an acute bout of aerobic exercise performed in the overnight-fasted state augments whole-body exercise fat oxidation and promotes Type I fibre IMTG utilisation as compared to fed state. However, given that IMTG contents were similar at post-exercise in both conditions, the use of fasted-state exercise to increases IMTG utilisation in these populations cannot be confirmed based on present observations.

D1.P6. Effects of short-, medium- and long-term resistance exercise programmes on measures of cardiometabolic health in adults: a systematic review and meta-analysis

\section{RUTH ASHTON ${ }^{1 *}$, GARRY TEW ${ }^{1}$, JONATHAN ANING ${ }^{2}$, STEPHEN GILBERT ${ }^{1}$, LIANE LEWIS ${ }^{3}$ \& JOHN SAXTON ${ }^{1}$}

${ }^{1}$ Department of Sport, Exercise \& Rehabilitation, University of Northumbria at Newcastle, ${ }^{2}$ Department of Urology, Freeman Hospital, ${ }^{3}$ School of Computer \& Information Science, University of Strathclyde ${ }^{*}$ Corresponding author: ruth.ashton@northumbria.ac.uk @ruth_ashton1

The aim of this study was to systematically review and summarise the intervention effects (via meta-analyses) of studies that have measured cardiometabolic health outcomes following short-, medium- and long-term programmes of resistance exercise training (RET). Studies were included in the review if they were a randomised controlled trial, compared RET programme of $>2$ weeks in duration alone, with a non-exercising or usual care group, recruited participants $>16$ years old and reported $\geq 1$ of the following outcomes: aerobic capacity, flow-mediated dilatation, C-reactive protein, total cholesterol, high-density lipoprotein cholesterol, low-density lipoprotein cholesterol, triglycerides, fasted glucose, fasted insulin, insulin resistance, blood pressure, mean arterial pressure, resting heart rate or clinical end-points. MEDLINE and Cochrane Library databases were searched from inception to February 2017. Risk of bias was assessed using the Cochrane Risk of Bias tool. A total of 180 trials were included involving both health and clinical populations. Systolic blood pressure was reduced following medium- (6-26 weeks) and longterm (>26 weeks) RET $(\mathrm{n}=1588$, mean difference $3.56 \mathrm{mmHg}[95 \% \mathrm{Cl}:-5.30,-1.82], P<0.001$ and $\mathrm{n}=529$, $-5.26 \mathrm{mmHg}[-9.12,-1.40], P=0.008$, respectively), as was diastolic blood pressure $(\mathrm{n}=1550,-1.72 \mathrm{mmHg}[-2.74$, $-0.69], P=0.001$ and $\mathrm{n}=509,-6.10 \mathrm{mmHg}[-9.29,-2.92]$, $P=0.0002$, respectively). Medium-term RET also led to reductions in fasted insulin $(n=674$, standardised mean difference $-0.68[-1.02,-0.33], P=0.0001)$ and insulin resistance $(\mathrm{n}=200$, mean difference $-1.92 \mu \mathrm{U} / \mathrm{ml}[-3.20,-$ $0.64], P=0.003)$. None of the included studies reported cardiovascular mortality, all-cause mortality or non-fatal 
endpoints and there were no exercise-related serious adverse events, although some studies reported transient levels of muscle soreness following RET. In conclusion, this systematic review provides quantitative estimates of the effect of RET on indices of cardiometabolic healthy in adults. RET appears a safe mode of exercise in both healthy and clinical populations and effective for inducing improvements in several measures of cardiometabolic health. The optimal design of a RET programme for improvements in cardiometabolic health is unknown and warrants further research. Additionally, future research should investigate the effects of RET on cardiovascular health and risk factor management in middle-aged and older clinical populations ( $>50$ years of age) in both supervised and home-based settings, and include long-term clinical end-points.

\section{D1.P7. Identifying barriers to physical activity and exercise for type 2 diabetes patients}

\section{ALISON CONNAUGHTON ${ }^{1 *} \&$ STEPHEN MELLALIEU ${ }^{2}$}

\section{${ }^{1}$ University of Wales, Trinity Saint David, ${ }^{2}$ Cardiff \\ Metropolitan University \\ *Corresponding author: a.connaughton@uwtsd.ac.uk}

Exercise can positively impact the prevention and delay of developing type 2 diabetes in high-risk adults (Nakanishi, Takatorige, \& Suzuki, 2004, Diabetologia, 47, 1768-1775). Despite this, type 2 diabetes patients generally do not meet the requirements of regular physical activity (Zhao, Ford, Li, \& Mokdad, 2008, Diabetic Medicine, 25, 221-227). Self Determination Theory (SDT: Deci \& Ryan, 2000, Psychological Inquiry) proposes that self-determined behavioural regulation is associated with positive behavioural and affective outcomes. When SDT is applied to physical activity/exercise, autonomous motivation has been linked to the adoption of, and adherence to exercise (Teixeira et al., 2012, International Journal of Behavioural Nutrition and Physical Activity, 9, 78). The aim of this study was to identify the barriers to physical activity and exercise within a type 2 diabetes population. NHS ethical approval was obtained (Ref:11/WA/0302) and a semi-structured interview guide developed to elicit the barriers type 2 diabetes patients perceive to partaking in physical activity and exercise. 12 males and 7 females (mean age: 65.83 $\pm 10.61 \& 66.29 \pm 15.81$ ) medically diagnosed with type 2 diabetes and resident in Wales (UK) were interviewed. The transcripts were inductively analysed and then deductively categorised using the constructs of SDT (Deci \& Ryan, 2000). Specifically, 89 barrier statements were identified and hierarchically ordered into 11 themes, which were then negatively categorised under competence, relatedness and autonomy (i.e., lack of). Three themes related to competence: Unable to demonstrate competence (e.g., lack of accountability to health professionals); Concerns over physical competence to exercise (e.g., fear of injury/pain) and; Concerns over competence to be able to exercise (e.g., lack of confidence). Three themes categorised under relatedness: Exercise not important (e.g., lack of emphasis on exercise at diagnosis), Lack of relatedness to other exercisers, and; Influence of childhood experiences (e.g., negative school PE/sport experiences). Five themes related to autonomy: Choosing other activities (e.g., work or competing activities); Work constraints (e.g., unconventional work hours); Practical reasons (e.g., preferred activities unavailable); Psychological barriers (e.g., internal battle to initiate exercise), and; Family roles and responsibilities (e.g., poor health of family members). The results suggest that barriers to exercise decrease perceptions of competence, relatedness and autonomy, and appropriate interventions should be implemented to assist type 2 patients to overcome such barriers and encourage more active and healthier lifestyles. Finally, these findings could form the basis for the development of a measure of barriers to exercise for type 2 diabetes patients.

D1.P8. A mixed methods approach to advance the understanding of physical activity behaviour during pregnancy

\section{MARLIZE DE VIVO* \& HAYLEY MILLS}

\section{Canterbury Christ Church University *Corresponding author: \\ m.de-vivo319@canterbury.ac.uk @marlizedv}

An active pregnancy encompasses benefits that apply to both mother and baby. However, before investing resources in interventions aimed at changing or supporting expectant mothers' physical activity behaviours, it is necessary to have a greater fundamental understanding of the modifiable factors influencing women's decisions during this time. Therefore, the main purpose of this study was to investigate the socio-cognitive determinants of physical activity during pregnancy. A mixed methods research approach using a multiphase design framed by the Theory of Planned Behaviour (TPB; Ajzen, 1991, Organizational Behavior and Human Decision Processes, 50, 179-211) guided the investigation. Following ethical approval and permission to carry out the study, participants were recruited from randomly selected antenatal clinics in the East Kent region of England. Application of the TPB required both qualitative and quantitative cross-sectional data to be collected. Study One $(n=18)$ involved the elicitation of pregnant women's beliefs towards being physically active. The modal salient behavioural, normative and control beliefs were used to inform the development of a TPB questionnaire which was implemented during the next phase of the investigation. Study Two $(n=78)$ examined the predictive utility of the TPB in explaining pregnant women's physical activity intentions and behaviour and scrutinised the role of past behaviour within this context. Finally, Study Three $(\mathrm{n}=10)$ comprised semi-structured interviews involving community midwives. Meta-inferences maintained the function of improving the validity of the study and advancing the overall aim of the research project. Specifically, triangulation was used to ascertain convergence, corroboration, and correspondence of findings between studies. Whilst results of the individual studies hold merit, meta-inferences point towards the development of three main themes: (1) pregnant women lack access to the necessary information that would allow them to make informed decisions regarding their engagement in physical activities; (2) a co-ordinated 
effort involving interprofessional collaboration is required to support pregnant women in overcoming barriers associated with regular physical activity participation; and (3) profiling pregnant women according to motivation and behaviour status could serve as a useful and manageable starting point for intervening to produce positive changes in pregnant women's physical activity behaviour. In normalising physical activity during pregnancy and achieving better health outcomes for mothers and babies, it is necessary to consider the modifiable factors involved in behaviour change, identify opportune moments to intervene, and involve a network of professionals in facilitating and supporting pregnant women's engagement with physical activities.

\section{D1.P9. Physical activity and mental wellbeing levels of university student nurses}

\section{AUDREY DUNCAN*, HELEN COLLINS, LINDA MCSWIGGAN, THOMAS MCCONNACHIE, GINNY HENDERSON, MARTYN JONES \& MICHAEL GALLACHER}

\section{University of Dundee \\ *Corresponding author: a.t.duncan@dundee.ac.uk}

In Scotland, 37\% of adults fail to meet the UK Physical Activity Guidelines (Brown et al., 2016, The Scottish Health Survey 2015. The Scottish Government) and life transitions, including from school to University, have been associated with a reduction in physical activity (PA) levels. It is clear that University students are an important target in preventing sedentary adulthood, improving health and reducing the prevalence of physical inactivity related disease. The aim of this project therefore, was to evaluate the PA and mental wellbeing levels of a student population. With institutional ethics approval, 106 first-year university student nurses were recruited (9 males, 97 females; age: $26 \pm 8$ years; stature: 1.66 $\pm 0.07 \mathrm{~m}$; body mass: $71.3 \pm 15.8 \mathrm{~kg}$ ) (mean $\pm \mathrm{s}$ ). Following informed consent and measurement of stretch-stature and body mass, PA levels were evaluated using the International Physical Activity Questionnaire (IPAQ) short form and mental wellbeing using the Warwick Edinburgh Mental Wellbeing Scale (WEMWBS). Results were analysed according to agerelated classifications (with 15 participants aged $\leq 18$ years classified as "children" for PA and body mass index (BMI) levels). $43.3 \%$ of participants were categorised as overweight/obese according to their BMI (mean 25.7, s $=5.4$ $\mathrm{kg} \cdot \mathrm{m}^{-2}$ ), comparable to age-related national data of $42 \%$ of the population. The mean WEMWS score was $49.0(s=7.8)$, also comparable to age-related national data (mean 49.1, $\mathrm{s}=$ $0.5)$. However, only $50 \%$ of participants met the moderate to vigorous PA (MVPA) guidelines, according to their selfreported IPAQ data, lower than the age-related national comparison of $75 \%$ (Brown et al., 2016). Additionally, only $8 \%$ of participants could correctly detail the UK recommended MVPA guidelines. Two recent studies reported that $69 \%$ of Scottish nurses were overweight/obese, higher than any other healthcare or non-healthcare profession (Kyle et al., 2016, International Journal of Nursing Studies, 53: 126-133) and that there are high rates of adjustment and mental health difficulties in student nurses (Horgan et al., 2016, The Journal of Advanced Nursing, 72: 3081-3092). While this study's results suggest that this cohort of students are overall following national trends in mental wellbeing and weight status, they are falling below national trends in physical activity levels, highlighting that they would benefit from early targeted intervention to increase MVPA levels. This has the potential to improve not only their own health but, as front-line healthcare professionals, the health of the patients that they interact with.

\section{D1.P10. The impact of exercise on postural sway in middle-aged overweight and obese women}

\section{EMMA EYRE*, CHRISTOPHER COOPER, LIAM AKHURST, MICHAEL DUNCAN \& JASON TALLIS}

\section{Coventry University \\ *Corresponding author: Emma.Eyre@coventry.ac.uk emma_eyre2}

The ability to maintain postural control is a fundamental functional skill required to prevent falls and complete tasks of daily living (Normand et al., 2013, Current Obesity Reports, 2, 235-240). Evidence has demonstrated that obesity induced changes in anthropometry have a significant impact on the ability of the body to maintain balance. Given that overweight/obese individuals are encouraged to engage in regular physical activity, the present study sought to examine the effects of short duration moderate intensity activity on postural sway in overweight/obese, middle aged women. Following instructional ethics approval, 18 participants were recruited (Mean \pm SD. Age $47 \pm 6$ ) and categorised into a normal weight and overweight/obese group based on BMl and again by body fat percentage (Mean \pm SD. BMI $21 \pm 2$ and $29 \pm 3$; body fat \% $27 \pm 5$ and $39 \pm 3$ for lean and overweight/obese group respectively), which was measured using multi frequency bioelectrical impedance analysis (TANITA MC-780, TANITA, Japan). Bipedal and unipedal assessments of postural sway were measured during a 30 second static balance assessment on both a hard and compliant surface, with eyes open and eyes closed, using a force platform (AMTI, AccuGait, Watertown, MA, $100 \mathrm{~Hz}$ ). Postural sway assessments were performed prior to and immediately following $10 \mathrm{~min}$ of treadmill exercise at $60 \%$ heart rate reserve. This exercise duration and intensity was chosen to align with the physical activity guidelines for adults which stipulate that moderate activity should be accumulated in bouts of at least 10 min (Department of Health, 2011, Department of Health). Following a mixed methods ANOVA, the results indicate that irrespective of body fat and BMI categorisation, postural sway (COP length, velocity, ellipse area, maximal anterior-posterior sway and maximal medial-lateral sway) was affected by condition (eyes open, eyes closed for bilateral and eyes open unipedal) $(P<0.01)$. However, postural sway was not significantly different between the lean and overweight/obese group or pre and post exercise $(P>0.05)$. Participants were unable to complete assessments of unipedal sway in the eyes closed condition on either a hard or compliant. This did not 
appear to differ between the normal weight and overweight/obese groups. As a result, these results confirm that the minimal guidelines for moderate physical activity do not affect static postural control in middle aged, overweight/obese women.

\section{D1.P11. The effects of acute high intensity exercise on pulse wave velocity and wave reflection characteristics in females.}

\section{KATHRYN HOLLOWAY* \& SAFRON NEWHOUSE}

\section{Liverpool Hope University \\ *Corresponding author: hollowk@hope.ac.uk}

Intensity of exercise has been shown to be a key contributor to alterations in central arterial stiffness (Tordi et al., 2010, European Journal of Applied Physiology, 108, 801-809). However, few studies have investigated these effects using repeated bouts of high intensity exercise $(\mathrm{HIE})$, with no studies in females. With gender differences in arterial distensibility, it is important to ascertain the effect acute HIE may have on arterial stiffness in females. Therefore, the purpose of this study was to ascertain whether an acute bout of HIE influenced pulse wave velocity and wave reflection characteristics in females. With institutional ethical approval, 8 healthy females (mean age $20 \pm 1$ years) took part in the study. The HIE was completed on a stationary lode cycle ergometer, consisting of 5 bouts of $1 \mathrm{~min}$ at $90 \% \mathrm{VO}_{2 \max }$ followed by $2 \mathrm{~min}$ of active rest at $60 \% \mathrm{VO}_{2 \mathrm{max}}$. Brachial blood pressures, along with wave reflection characteristics, were measured using pulse wave analysis. Arterial stiffness was measured using carotid-femoral pulse wave velocity (cfPWV). Measurements were taken before, immediately ( $<5 \mathrm{~min})$, $30 \mathrm{~min}$ and $1 \mathrm{~h}$ post HIE. There was a significant decrease in systolic blood pressure from immediately after exercise until $30 \mathrm{~min}(P<0.05)$ post exercise. There was a significant decrease in augmentation pressure $(P<0.05$, partial $\left.\eta^{2}=0.609 ; A P\right)$ and thus augmentation index (Alx) at 30 $\min (P<0.05)$ and $1 \mathrm{~h}$ post $\operatorname{HIE}\left(P<0.05\right.$, partial $\eta^{2}=$ $0.594)$. However, there was no significant difference $(P>$ $0.05)$ in pulse pressure (AP), systolic pressure (SP), central diastolic pressure (CDP) or mean arterial pressure (MAP) at any time point when compared to baseline measures. Ultimately, there was no significant change in CPPWV in response to a 15-min bout of HIE. The data demonstrates that a high intensity exercise bout with 2-min active rest periods can decrease systemic arterial stiffness but has no significant effect on female's overall vascular function. Therefore, practitioners should not be concerned with implementing repeated bouts of HIE in females, as it does not cause a detrimental effect on the vasculature, and in effect is beneficial in reducing systemic arterial stiffness post exercise.
D1.P12. Comparing the validity of two aerobic capacity field tests for use with obese Emirati males enrolled in National Service

\section{RICHARD JOHNSTON ${ }^{1 *}$, GARETH PICKNELL ${ }^{2}$, GRAHAM $\mathrm{NICHOLSON}^{2}$ \& MOUZA AL SHEHHI ${ }^{1}$}

\section{${ }^{1}$ 3Dimensions Physical Performance LLC, ${ }^{2}$ United Arab Emirates Armed Forces \\ *Corresponding author: richard.johnston@3ds.ae}

Wide consensus suggests that interventions aimed at reducing body composition measures and increasing cardiorespiratory fitness are worthwhile endeavours for protecting individuals against related diseases and early death (Hogstrom et al., 2016, International Journal of Epidemiology, 45, 1159-1168). Given the importance ascribed to these relationships, accurately monitoring outcome measures following intervention is crucial for evaluating their effectiveness. Indeed, body composition measures have been validated extensively with diverse population groups for evaluating weight management. Whereas, less is known about the efficacy of physical fitness field tests; namely, the 6-min walk test (6MWT) and the Rockport walk test (RWT), as valid predictors of aerobic capacity across these varied settings. Therefore, the aim of this study was to compare the validity of these two functional walking tests to determine whether they are useful for monitoring physical fitness of obese Emirati males enrolled in National Service. With institutional ethics approval, 23 participants (mean age: $28.0 \pm 7.4$ years; BMl: $35.6 \pm 3.5$ ) completed three aerobic capacity tests: (i) a treadmill based $\mathrm{VO}_{2}$ peak test using Fitmate Pro (COSMED, Italy), following the Modified Bruce Protocol, (ii) a RWT that required participants to complete a best effort walk covering 1609 meters, and (iii) a $6 \mathrm{MWT}$ that required participants to cover as much distance as possible during a 6-min period. For both field tests, relevant equations for calculating predicted aerobic capacity were used. Significant correlations were noted between Fitmate Pro and RWT $(r=0.64 ; P<0.01)$, and between Fitmate Pro and 6MWT $(r=0.72 ; P<0.01)$, whilst Bland and Altman agreement analysis indicated mean differences of $1.43(95 \%$ $\mathrm{Cl}=-0.55$ to 3.42$)$ and $-1.23(95 \% \mathrm{Cl}=-2.96$ to 0.51$)$ respectively. RWT generally under predicted $\mathrm{VO}_{2}$ peak by $4.05 \%$, whilst the 6MWT generally overestimated $\mathrm{VO}_{2}$ peak by $3.44 \%$. Further, Limits of Agreement were noted as 10.45 to -7.61 for considering agreement between Fitmate Pro and RWT, and 6.63 to -9.09 for considering agreement between Fitmate Pro and 6MWT. Collectively the results indicate that both the 6MWT and RWT are adequate for predicting aerobic capacity of obese Emirati males. Given the advantages of the 6MWT; namely, it is simple to conduct, can be performed indoors, requires minimal equipment, and has a short test duration, the authors recommend the 6MWT as the test of choice when using a functional walk test for clinical or research purposes with this population (Solway et al., 2001, Chest, 119, 256-270). 
D1.P13. Individual differences in affective responses to and intention to repeat low volume high intensity interval exercise

\section{AILSA NIVEN*, CLAIRE BRADLEY \& SHAUN PHILLIPS}

\section{University of Edinburgh \\ *Corresponding author: ailsa.niven@ed.ac.uk @ailsaniven}

There has been a growth in research examining affective responses to High Intensity Interval Exercise (HIIE) because these responses can provide insight into the acceptability of, and likelihood of adherence to HIIE. However, findings to date have been mixed and few studies have considered how individual differences may moderate affective responses. The aim of this study was to examine the influence of the dispositional trait of exercise tolerance on affective responses to and intention to repeat low volume HIIE. Following institutional ethical approval, healthy participants $(n=114)$ provided informed consent and initially completed the Preference for and Tolerance of Exercise Intensity Questionnaire. From this sample, high tolerance $(\mathrm{HT} ; \mathrm{n}=$ 19 ; male $=7 ;$ mean age $=20.37 \pm 1.46$ ) and low tolerance $(\mathrm{LT} ; \mathrm{n}=17$; male $=5 ;$ mean age $=21.8 \pm 2.0)$ groups were identified. Both groups completed $10 \times 6$ sec cycle sprints on a mechanically braked ergometer against a resistance of $7.5 \%$ (males) or $6.5 \%$ (females) body mass, interspersed with $60 \mathrm{sec}$ passive recovery. Affective valence and perceived activation were recorded before exercise, immediately after sprints 2, 4, 6, 8, and 10, and $20 \mathrm{~min}$ postexercise. Participants' intentions to repeat HIIE 1 and 3 times per week were assessed $20 \mathrm{~min}$ and $24 \mathrm{~h}$ post-HIIE. Affective valence significantly decreased across the sprints in both groups $\left(P=0.019, \eta_{p}^{2}=0.103\right)$ and was significantly different between groups $\left(P=0.018, \eta_{p}^{2}=0.154\right)$. Affective valence was significantly lower in LT at sprint $2(P=0.022, \mathrm{~d}$ $=0.94)$ and moderate to large effect sizes $(d=0.66-0.94)$ were found at each time point. Perceived activation significantly increased across sprints in both groups $\left(P<0.001, \eta_{p}^{2}\right.$ $=0.497$ ), with no significant between-groups differences $(P=$ $\left.0.174, \eta_{p}^{2}=0.054\right)$. At $20 \mathrm{~min}$ and $24 \mathrm{~h}$ post-exercise, respectively, the HT group had significantly greater intentions to repeat HIIE once per week $(P=0.002, d=1.20 ; P=0.003, d=$ 1.18) and three times per week $(P<0.001, d=1.32 ; P=$ $0.002, d=1.03$ ). Affective responses to HIIE are influenced by the dispositional trait of exercise tolerance, with LT participants experiencing more negative affect and lower intentions to repeat HIIE than HT participants. Future research should consider the influence of these responses on subsequent behaviour. These findings suggest that HIIE is unlikely to be experienced positively by everyone, supporting the personalisation of exercise prescription.
D1.P14. Associations of cardiorespiratory fitness with cardiovascular disease, respiratory disease and cancer, and all-cause mortality: findings from the UK Biobank prospective cohort study
ANNE SILLARS, LEWIS STEELL, HUI LI, FANNY PETERMANN, NAVEED SATTAR, JILL PELL, STUART GRAY, JASON GILL \& CARLOS CELIS-MORALES*

\section{University of Glasgow}

*Corresponding author: carlos.celis@glasgow.ac.uk @carloscelism

Although cardiorespiratory fitness is a strong predictor of all-cause and CVD mortality, there is limited evidence regarding its association with other health outcomes (Ross et al., 2016, Circulation, 135, 1-48). This study aimed to investigate the association of fitness with all-cause mortality, and cardiovascular, respiratory and cancer outcomes. 74,836 participants (54.0\% women) with available fitness data from the prospective UK Biobank cohort were included in this study. Cardio-respiratory fitness was the exposure of interest and it was measured using a previously validated 6min incremental ramp cycle ergometer test (Celis-Morales et al., 2017, European Heart Journal, 38, 116-122). Cardiovascular disease, respiratory disease, and cancer incidence and mortality, and all-cause mortality were the outcomes. Associations between fitness and these outcomes were investigated using Cox proportional hazard models. This study was granted ethical approval. The main results shows that 1476 participants died, and there were 3586 cardiovascular incidents (or events), 1242 respiratory and 3361 cancer events, over a median of 7.0 years [IQR 6.37.7] follow-up. Participants in the moderate (20th-59th centile) and high fitness ( $\geq 60$ th centile) categories had a lower hazard for all-cause mortality (HR: 0.69 [95\% Cl: 0.60-0.78], and 0.66 [95\% Cl: $0.57-0.76]$, respectively) compared to those with low fitness (<20th centile). Similar findings were observed for incident cardiovascular (HR: 0.63 [0.58$0.69]$ and 0.66 [0.60-0.72]), chronic obstructive pulmonary disease (HR: 0.65 [0.43-0.98], and 0.52 [0.31-0.88]), all respiratory disease (HR: 0.72 [0.63-0.83], and 0.67 [0.60$0.72]$ ), and colorectal cancer (HR: 0.85 [0.63-1.14], and 0.71 [0.51-0.98]). However, no associations were found with breast, prostate or lung cancer. These associations were independent of measured sociodemographic, comorbid, dietary and physical activity factors and results did not change after performing a 2-years landmark analysis. Our results suggest that cardiorespiratory fitness is associated with risk of respiratory disease and colorectal cancer, as well as all-cause mortality and CVD. If this relationship proves to be causal, which seems probable, populations should be informed that increasing their level of fitness can reduce their risk of a range of important health outcomes. 


\section{Day 1. Posters - Physiology and Nutrition}

\author{
D1.P15. Physiological impact of hot fire training \\ on UK firefighters: implications for the ageing fire- \\ fighter
}

\section{ELISABETH BOARD \& TIMOTHY HILL}

\section{University of Sunderland \\ *Corresponding author: lisa.board@sunderland.ac.uk}

Strenuous physical activity and severe heat may increase the risk of an adverse cardiovascular event or sudden cardiac death $(S C D$.) Risk increases with age, and with exercise intensity. Heat stress, dehydration and strenuous physical exertion are ubiquitous concerns for firefighters. Previous research determined the physical demands of firefighting under normal environmental conditions, but not during live firefighting. The physiological impact of live firefighting manoeuvres in extreme heat, across different age groups, was unknown. Therefore the purpose of this study was to explore the impact of search and rescue firefighting activities during live fire training in extreme heat and smoke on cardiovascular responses, with a specific focus on the ageing firefighter. With institutional ethical approval 16 firefighters (15 male; age, 23-59 years) were conveniently recruited. Participation was voluntary. Mandatory minimum criteria for aerobic capacity $(42 \mathrm{ml}$. $\mathrm{kg}^{-1} \cdot \mathrm{min}^{-1}$ ) using a Chester Step Test protocol were met. Firefighter training took place at the Fire and Rescue Training Centre, Newcastle Airport. Each firefighter was exposed to three firefighting exposures over a one-day training course: Cold (smoke only), and two hot conditions (fire $\left[150-350^{\circ} \mathrm{C}\right]$ and smoke). Each exposure lasted $25-30$ min. Firefighters, in pairs, searched a building, hauling a fire-hose, to locate and recover a $70 \mathrm{~kg}$ dummy. Heart rate (HR) was recorded continuously (Zephyr ${ }^{\circledR}$ Bio-harness). Peak HR, peak HR expressed as a percentage of age-predicted maximal $H R\left(H R_{\max }=220\right.$-age $), H R$ recovery in $60 \mathrm{~s}$ $\left(\mathrm{HRR}_{60}\right)$, ratings of perceived exertion (RPE) and thermal comfort (TC; scale 1-5, "no discomfort" to "maximal discomfort") were recorded. Supramaximal HR responses, defined as a peak HR greater than the age-predicted $\mathrm{HR}_{\max }$, were observed in $81 \%$ and $50 \%$ of firefighters in the hot-fire and cold-smoke scenarios respectively. Peak $\mathrm{HR}$ responses exceeded age-predicted $\mathrm{HR}_{\max }$ in both young $\left(\% \mathrm{HR}_{\max }, 104 \%\right)$ and old $\left(\% \mathrm{HR}_{\max }, 108 \%\right)$ firefighters $(P>0.05)$. RPE and thermal discomfort were elevated in the hot-fire exposure compared to the cold-smoke exposure (RPE: $17 \pm 2$ and $14 \pm 3$ units, $d=1.2 ;$ TC: $4.5 \pm 0.5$ and $3.0 \pm 0.5$ units respectively, $d=3.0)$. HRR ${ }_{60}$ was attenuated in older firefighters compared to younger firefighters (coldsmoke: $15 \pm 5$ b. $\mathrm{min}^{-1}$ and $21 \pm 6$ b. min ${ }^{-1}, d=-1.09$; hotfire: $11 \pm 6 \mathrm{~b} \cdot \mathrm{min}^{-1}$ and $16 \pm 8 \mathrm{~b} \cdot \mathrm{min}^{-1}, d=-0.71, P=$ 0.02). Simple regression analysis was used to predict peak HR from age during firefighting activities in extreme heat (Peak HR = 227.006-1.044 [age]; SEE 15.4; $\mathrm{R}^{2}=0.37 ; P=$ 0.012). Preliminary findings suggest older firefighters may have an increased risk for a cardiovascular event, particularly in the hot-fire conditions, however further research is required.

D1.P16. Changes in the internal load training and fatigue of young athletes taking carbohydrate and protein $2 \mathrm{~h}$ before training

\section{NOELIA BONFANTI ${ }^{1 *} \&$ KRIZIA RADESCA ${ }^{2}$}

${ }^{1}$ San Jorge University, ${ }^{2}$ European University of Madrid *Corresponding author: noeliabonfanti@yahoo.com.ar bonfanti_noelia

Young athletes use to avoid lunch intake when they train in the afternoon but, a small volume rich carbohydrate $(\mathrm{CHO}) /$ protein (PRO) intake before training would be able to supply the nutrients avoided at lunch. Consequently, the internal load training and fatigue could decrease allowing athletes to endure the external load training planned by coaches to improve performance with an appropriated recovery between sessions. Thus, the aim of this study was to investigate the possible changes that a pre-training snack intake could produce in the internal load training and fatigue of young athletes. Methods: With European University of Madrid ethics approval a 7weeks experimental study was carried out with 26 Spanish young healthy runners (13-16 years) whose were randomized into an experimental group $(\mathrm{EG})$ or a control group (CG). EG took a CHO/PRO snack (1 g CHO/Kg body weight $(\mathrm{BW})+0.25 \mathrm{~g} \mathrm{PRO} / \mathrm{Kg} \mathrm{BW}$ ) and water (5-7 ml/Kg BW) $2 \mathrm{~h}$ before training while CG did not take any foods and only drank water $(5-7 \mathrm{ml} / \mathrm{Kg} \mathrm{BW})$. Before and after the intervention a 3-day food diary was recorded and body composition was measure (ISAK protocol). Pre and post training, internal load (S-RPE, 0-10 scale) and fatigue (FA) (1-10 scale) were recorded by athletes. A pre and post intervention group comparison was done by an independent T-Test and U-Mann-Whitney test for parametric (PV) and non PV respectively. Before and after intervention, each group were compared using paired T-Test (PV) and Wilcoxon test (NPV). The confidence intervals for these results was $95 \%$. Results: After the intervention, internal load training (S-RPE) decreased in EG (day 1: $4.5 \pm 1.3$ vs $3.3 \pm 1.6 ; \mathrm{t}(12)=6.1 ; P<0.05$ ) and increased in $\mathrm{CG}$ (day 1 : $4.1 \pm 1.4$ vs $5.3 \pm 1.4 ; \mathrm{t}(12)=-7.4 ; P<0.05)$ with significant differences between groups (day 1: $5.3 \pm 1.4$ vs $3.3 \pm 1.6$; $\mathrm{t}$ $(24)=4.1 ; P<0.05$ ). FA pre-training increased in CG (day 1 : $1.2 \pm 0.4$ vs $2.0 \pm 1.0 ; Z=-2.7 ; P<0.05 ;$ day $2: 2.2 \pm 0.9$ vs $2.7 \pm 1.3 ; Z=-2.1 ; P<0.05$ ) and was higher than $E G$ (day $1: 2.0 \pm 1.0$ vs $1.2 \pm 0.4 ; Z=-2.2 ; P<0.05 ;$ day $2: 2.7 \pm 1,3$ vs $1,3 \pm 0.5 ; Z=-3.1 ; P<0,05)$. EG also decreased FA after training (day $1: 5.1 \pm 1.3$ vs $3.9 \pm 1.4 ; \mathrm{t}(12)=4.2 ; P<0.05$ ). Conclusion: the intake of a pre-training snack could supply the glucose needed by the brain avoiding the deterioration of the central nervous system function during exercise and improving glycogen storage. Consequently, the 
internal load training and fatigue after training of athletes would be reduce while its omission would increase fatigue before training.

\section{D1.P17. The effect of plasma volume on changes in biomarkers of muscle damage, inflammation, oxidative stress and leukocyte differential following a $42.2 \mathrm{~km}$ trail run, and the efficacy of recovery interventions}

EMMA COCKBURN ${ }^{2 *}$, FRANK HILLS ${ }^{1}$, LAURA WILSON ${ }^{1}$, KATHRYN PAICE ${ }^{1}$, ALYSSIA WOOD ${ }^{1}$ \& LYGERI DIMITRIOU ${ }^{1}$

\section{${ }^{1}$ Middlesex University, ${ }^{2}$ Newcastle University *Corresponding author: e.cockburn@mdx.ac.uk @cockburn23}

Exercise induces changes in plasma volume (PV), therefore, accounting for this when investigating the biological response of circulating biomarkers is important. Biomarkers are used to investigate the efficacy of recovery interventions with few researchers correcting for PV changes. Therefore, the aim of this study was to examine whether PV affects a) the magnitude of change over time in biomarkers; b) outcomes of popular recovery strategies. With ethical approval 32 endurance-trained males (age $39 \pm 8$ years; height $1.78 \pm$ $0.08 \mathrm{~m}$; mass $77.0 \pm 10.4 \mathrm{~kg}$ ) completed a $42.2 \mathrm{~km}$ run. Participants were randomly assigned to one of 3 interventions (placebo $(\mathrm{PL})$; cherry juice (CJ); cold water immersion $(\mathrm{CWI})$ ). Markers of inflammation (CRP, IL-6, TNF-a), muscle damage (CK), oxidative stress (PC, TBARS, SOD) and leukocyte differential were measured at baseline (B), immediately, $24 \mathrm{~h}$ and $48 \mathrm{~h}$ post run. Biomarkers were adjusted for PV changes (Dill \& Costill, 1974, Journal of Applied Physiology, 37, 247-248). Data were analysed using magnitude-based inferences for the difference between the change over time for uncorrected (Un) and corrected (Co) values for each biomarker (difference; $\pm 90 \% \mathrm{Cl}$ effect size), and the difference between each recovery intervention using both values. CoCK B-post, B-24 $\mathrm{h}$ and B-48 $\mathrm{h}$ were possibly $(0.23 ; \pm 0.33)$, likely $(0.43 ; \pm 0.34)$ and possibly $(0.27 ; \pm 0.32)$ greater than Un-CK. Co-neutrophil and co-eosinophils B-24 h and B-48 $\mathrm{h}$ were likely greater than Un-neutrophils $(24 \mathrm{~h}: 0.25 ; \pm 0.07 ; 48 \mathrm{~h}$ : $0.27 ; \pm 0.08$ ) and Un-eosinophils ( $24 \mathrm{~h}: 0.23 ; \pm 0.07 ; 48 \mathrm{~h}: 0.24$; \pm 0.07). Co-lymphocytes B-24 $\mathrm{h}$ and B-48 were very likely greater than Un-lymphocytes $(24 \mathrm{~h}: 0.33 ; \pm 0.10 ; 48 \mathrm{~h}: 0.36$; \pm 0.10 ). Co-monocyte $\mathrm{B}-24 \mathrm{~h}$ and $\mathrm{B}-48 \mathrm{~h}$ were possibly greater than changes in Un-monocyte ( $24 \mathrm{~h}: 0.20 ; \pm 0.06 ; 48$ h: $0.22 ; \pm$ 0.06). Differences between Un and Co values of neutrophils, lymphocytes, monocytes and eosinophils B-immediate were all trivial as were differences in Un and Co values of basophils, and inflammatory and oxidative stress biomarkers at all time points. Using corrected values altered the magnitude of difference and qualitative outcomes for comparisons of recovery interventions compared with uncorrected. For example, between PL and CWI CK B-24 h changed from unclear to possibly harmful, and lymphocytes B-24 h changed from possibly trivial to possibly harmful. In conclusion, correcting biomarkers for changes in PV affects the magnitude of change over time in CK and leukocyte differential. In some cases this alters the qualitative inferences that can be made about recovery interventions. Therefore, following a marathon circulating biomarkers should be corrected for changes in plasma volume.

D1.P18. Effect of exercise on appetite and appetite hormones in men with variants of the gastrinreleasing peptide gene rs7243357 polymorphism

\section{JAMES DORLING ${ }^{1}$, JENNY JONES ${ }^{2}$, ANDREA PUCCI ${ }^{2}$, JAMES KING ${ }^{1}$, RACHEL BATTERHAM ${ }^{2}$ \& DAVID STENSEL ${ }^{2 *}$}

\section{${ }^{1}$ Loughborough University, ${ }^{2}$ University College London *Corresponding author: D.J.Stensel@lboro.ac.uk}

The $\mathrm{T}$ allele of the gastrin-releasing peptide gene (GRP) rs7243357 single nucleotide polymorphism (SNP) is linked to an increased risk of obesity compared to the $G$ allele (Locke et al., 2015, Nature, 518, 197-206). Variations may be mediated by appetite and appetite hormones. Exercise suppresses appetite and increases total PYY (Broom et al., 2009, American Journal of Physiology - Regulatory, Integrative and Comparative Physiology, 296, R29-R35), yet the effect of exercise and GRP rs7243357 SNP on appetite and total PYY is not known. Thus, this study aimed to assess the effect of exercise on appetite and total PYY in males homozygous for the T-allele and G-allele of GRP rs7243357 SNP. After approval from the institution's ethics committee, 359 white males were genotyped for GRP rs7243357 SNP. From this cohort, $10 \mathrm{GG}$ and $10 \mathrm{TT}$ participants were recruited. First, participants completed an incremental treadmill test to determine maximal oxygen uptake. Participants then completed two lab-based trials, exercise and control, in a counterbalanced design. In the exercise trial, participants ran for 60 mins at $70 \%$ maximal oxygen uptake and then rested for $7 \mathrm{hrs}$, while participants rested for $8 \mathrm{hrs}$ in the control trial. A mixed test meal consisting of a sandwich, crisps, chocolate cake and a milkshake (1373 kcal) was consumed at $1.5 \mathrm{hr}$. Appetite ratings, assessed via visual analogue scales, and blood samples for the measurement of total PYY were taken frequently during each trial. Energy intake was measured at a buffet meal provided at $6.5 \mathrm{hr}$. Exercise suppressed appetite during the run $(P<0.001)$, although there was no effect of genotype on appetite $(P \geq 0.195)$. Neither exercise $(P=0.654)$ nor genotype $(P=0.581)$ affected energy intake at the buffet meal. While exercise increased concentrations of total PYY $(P<0.001)$, there was no main effect of genotype on total PYY $(P=0.604)$. However, there was a genotype-by-trial interaction, with the magnitude of total PYY elevation being greater in GGs than TTs in response to exercise $(37.0 \mathrm{pg} / \mathrm{ml}$ vs $24.0 \mathrm{pg} / \mathrm{ml} ; P=0.045)$. Exercise suppresses appetite, increases total PYY and does not cause compensatory changes in energy intake. Appetite and energy intake were similar between $\Pi \mathrm{Ts}$ and GGs. However, carriers of GG genotype show a greater elevation in total PYY after exercise. This may suggest appetite regulation in GGs may be more favourable than TTs after exercise, although further verification work is required. 
D1.P19. Low doses of caffeine produce individual and task-dependent responses in elite adolescent male soccer players

\section{MATTHEW ELLIS ${ }^{1 *}$, NEIL CLARKE ${ }^{2} \&$ MARK NOON ${ }^{2}$ \\ ${ }^{1}$ Coventry City FC, ${ }^{2}$ Coventry University \\ *Corresponding author: matthewellis529@gmail.com @MatthewLeeEllis}

Caffeine's ergogenic properties are thought to enhance neural activation of the muscle through the central nervous system (Spriet 2014, Sports Medicine, 44, 175-184). Large doses of $\sim 6 \mathrm{mg} \cdot \mathrm{kg}^{-1}$ body mass (BM) have improved performance during intermittent running, jumping, agility and passing protocols (Foskett et al., 2009, International Journal of Sports Nutrition and Exercise Metabolism, 19, 410-423). However, there is sparse data on low doses of caffeine $(\leq 3$ $\left.\mathrm{mg} \cdot \mathrm{kg}^{-1} \mathrm{BM}\right)$, especially in elite adolescent soccer players. The aim of the study was to investigate the ergogenic potential of caffeine in adolescent soccer players and to establish the most appropriate caffeine dose. Ten elite youth soccer players $(177.3 \pm 4.8 \mathrm{~cm}, 66.9 \pm 7.9 \mathrm{~kg}$ and $16 \pm 1$ years old $)$ participated in the study. All participants and parents provided written informed consent following institutional ethical approval. Participants consumed 1,2 or $3 \mathrm{mg} \cdot \mathrm{kg}^{-1}$ caffeine in a gelatin capsule or a $2 \mathrm{mg} \cdot \mathrm{kg}^{-1}$ placebo (PLA) in a single blind, randomised, crossover study design $1 \mathrm{hr}$ prior to testing. Testing consisted of a $20 \mathrm{~m}$ sprint, arrowhead agility (left and right), counter-movement jump (CMJ) in a non-fatigue and fatigued state and the Yo-Yo intermittent recovery test level-1 (IR1) on an indoor 3G synthetic surface. Data were analysed using a repeated measures analysis of variance and effect size calculated using partial eta squared $\left(\eta_{\mathrm{p}}{ }^{2}\right)$ which were defined as trivial $(<0.1)$, small $(0.1-0.3)$, moderate (0.3-0.5) and large (>0.5). When comparing caffeine to placebo, $20 \mathrm{~m}$ sprint time $\left(P=0.080, \eta_{p}{ }^{2}=0.218\right)$ and the arrowhead agility $\left(P=0.069, \eta_{p}{ }^{2}=0.227\right)$ displayed a small improvement. Fatigued CMJ performance resulted in a small improvement within the caffeine condition $\left(P=0.230, \eta_{p}{ }^{2}=\right.$ $0.145)$. Trivial improvements were also observed in the unfatigued CMJ $\left(P=0.915, \eta_{p}{ }^{2}=0.019\right)$ and Yo-Yo IR1 $(P=0.900$, $\left.\eta_{p}{ }^{2}=0.021\right)$ when comparing caffeine to placebo. Furthermore, $20 \mathrm{~m}$ sprint time displayed a small improvement between caffeine doses ( $P=0.041, \eta_{p}{ }^{2}=0.298$ ). However, there were no significant difference between caffeine doses for CMJ $\left(P=0.049, \eta_{p}{ }^{2}=0.006\right)$, agility $\left(P=0.785, \eta_{p}{ }^{2}=0.027\right)$, Yo-Yo IR1 $\left(P=0.944, \eta_{p}{ }^{2}=0.006\right)$ and CMJ pre and post Yo-Yo IR1 $\left(P=0.191, \eta_{p}{ }^{2}=0.168\right)$. Thus, results indicate that there are no significant differences between placebo and all caffeine doses with a range of trivial to small effect sizes. However, the trivial to small improvements in performance suggest an individualised approach to caffeine ingestion in youth soccer players should be considered. In conclusion, it is possible for low doses of caffeine to enhance physical performance in young soccer athletes but the responses are individual and task dependent.
D1.P20. Effect of elbow position on grip strength in children: validity and reliability of TKK 5101 and DynX dynamometers

\section{STEFAN KOLIMECHKOV*, LUBOMIR PETROV \& ALBENA ALEXANDROVA}

\section{National Sports Academy "Vassil Levski" \\ *Corresponding author: kolimechkov@gmail.com @kolimechkov}

One of the most widely used methods to assess upperbody isometric strength in children is the handgrip strength test. Owing to inconsistent findings, however, it is not clear which elbow position and which dynamometer type are most appropriate for achieving the maximal grip strength. Similar studies have been conducted on adolescents, whereas, studies in children are scarce. Therefore, the purpose of this study was to investigate whether elbow position and the type of dynamometer affect the handgrip strength in children. A total of 60 children, 6 to 11 years old ( 30 boys and 30 girls), from London participated in this study, and an ethics approval was obtained prior to the tests. Grip strength was measured by two different dynamometers: TKK digital hand dynamometer (TKK 5101 Grip-D, Takey, Tokyo, Japan) and DynX electronic hand dynamometer (MD System, Inc., Westerville, OH, USA). Every child performed two different tests with each type of dynamometer. The first test was with the elbow fully extended, and the second with flexed elbow at $90^{\circ}$. While using the TKK dynamometer, grip strength was significantly higher when the test was performed with elbow extended, in contrast to those obtained with flexed elbow (14.58 \pm $3.04 \mathrm{~kg}$ vs $12.97 \pm 2.99 \mathrm{~kg}, P<0.001$ for right hand, and $14.25 \pm 3.05 \mathrm{~kg}$ vs $12.61 \pm 2.99 \mathrm{~kg}, P<0.001$ for left hand). Furthermore, Cohen's effect size values $(d=0.87$ for right hand, and $d=0.91$ for left hand) suggested high practical significance. When using the DynX dynamometer, the difference between the two elbow positions was smaller $(13.84 \pm 3.22 \mathrm{~kg}$ vs $13.35 \pm 3.01 \mathrm{~kg}, P=0.035$ for right and, and $13.35 \pm 2.95 \mathrm{~kg}$ vs $12.77 \pm 2.96 \mathrm{~kg}, P=0.003$ for left hand), and the effect sizes were small to moderate ( $d=$ 0.28 and $d=0.41$, respectively). The validity and reliability of the TKK and DynX dynamometers were analysed by using known weights within the range of the children's strength $(5-30 \mathrm{~kg})$. The criterion-related validity analyses showed a systematic bias of $-0.20 \mathrm{~kg}(P<0.05)$ for the TKK, and $-0.42 \mathrm{~kg}(P<0.001)$ for the DynX dynamometer. The reliability analyses revealed a systematic bias of -0.07 $\mathrm{kg}$ in the TKK, and $0.10 \mathrm{~kg}$ in the DynX dynamometer $(P>$ 0.05 for both dynamometers). Both dynamometers provided sufficient results in terms of their validity and reliability, and can, therefore, be used when assessing handgrip strength in children. Performing the handgrip strength test with elbow extended appears to be the most appropriate protocol in order to evaluate maximal handgrip strength in children. 
D1.P21. A mixed-methods assessment of a multisensor wearable band during normoxia and hypoxia

DAVID J. MUGGERIDGE ${ }^{1,2^{*}}$, MIA BURLEIGH ${ }^{2}$, DAVID HUGHES ${ }^{2}$, KEVIN D. ROONEY ${ }^{2}$, STEWART WHITING ${ }^{3}$, JULIEN S. BAKER ${ }^{2} \&$ CHRIS EASTON ${ }^{2}$

\author{
${ }^{1}$ University of Strathclyde, ${ }^{2}$ University of the West of \\ Scotland, ${ }^{3}$ Snap 40 Ltd \\ ${ }^{*}$ Corresponding author: \\ david.muggeridge@strath.ac.uk \\ @davemuggeridge
}

Wearable devices in the sport and healthcare industries have grown exponentially in recent years as we move towards a personal measurement approach. Whilst this technology is not new, hardware that incorporates multiple sensor measures are limited. The PROTOTYPE used here aims to monitor up to seven "vital physiological signs" including heart rate $(\mathrm{HR})$ and oxygen saturation $\left(\mathrm{SpO}_{2}\right)$. Despite this, the validity of the measurements, and the usability of the device by practitioners, are currently unknown. The purpose of this study was to assess the validity of the PROTOTYPE to measure two of the key variables $\left(\mathrm{HR} \& \mathrm{SpO}_{2}\right)$ at rest in normoxia and hypoxia. The usability of the PROTOTYPE by practitioners was also evaluated. Following ethical approval, 44 healthy participants (31 \pm 9 years) completed one experimental trial (VAL) and 11 healthcare professionals completed one trial in a simulated ward (USE). During VAL, the PROTOTYPE was secured laterally on the upper-left arm and output from device sensors were recorded continuously using bespoke software. Reference gold standard (GS) outputs were recorded simultaneously $\left(\mathrm{HR}=\right.$ Polar $\mathrm{H} 7, \mathrm{SpO}_{2}=$ Masimo Pulse Oximeter). Participants then completed two experimental conditions $(T 1, T 2)$. During $T 1$, participants lay supine for $10 \mathrm{~min}$ in normoxia. During T2 participants were exposed to 5 min Hypoxia (Altium i10). Data were filtered to exclude data affected by movement and random artefacts. Concordance correlation coefficient (CCC), Bland and Altman plots and Wilcoxon rank test assessed the levels of agreement between the PROTOTYPE and the GS. During USE, participants were provided with video and written user guides before initiating the device on a participant. Sessions were video-recorded and participants completed a "user questionnaire" and focus group $(n=5)$. VAL highlighted substantial agreement between GS and PROTOTYPE variables (HR: CCC $=0.88$, SPO2: $C C C=0.68$ ). PROTOTYPE slightly overestimated HR by 2 beats $\cdot \mathrm{min}^{-1}(95 \% \mathrm{Cl}=-8-$ $12 \mathrm{bpm})$. The mean bias for $\mathrm{SpO}_{2}$ approached zero (1\%, 95\% $\mathrm{Cl}=-4-6 \%$ ). Evaluation of the questionnaire and focus group suggests a positive level of agreement between the experience and usability of the device, and it was concluded that the device is functional, usable and could be of major benefit to practice. These findings indicate that the PROTOTYPE device is capable of measuring $\mathrm{HR}$ and $\mathrm{SpO}_{2}$ within reasonable limits of GS measures at rest and appears functional in practice. Despite this, algorithms should be enhanced to improve the power for individual agreement. 


\author{
D1.P22. Patient motivation while completing \\ physical therapy
}

\section{DIANA AVANS*, DANYELLE ALLEN \& JASMINE FREEMAN}

\author{
${ }^{1}$ Vanguard University of Southern California \\ *Corresponding author: davans@vanguard.edu
}

Motivation is crucial to a patient's outcome in physical therapy. Integrating motivation therapy with physical therapy has shown to improve therapy outcomes (Vong et al., 2011, Archives of Physical Medicine and Rehabilitation, 92, 176-183). To create motivation strategies, patient motives need to be understood. The purpose of this study was to discover patient motives for physical therapy and if there were differences based on acute or chronic injury, athlete vs. non-athlete and gender. Informed consent, IRB and clinician approval were obtained. Men $(n=30)$ and women $(n=24)$ ranging in age from $18-35$ were recruited from 2 area clinics. Pelletier's (1997, Journal of Personality Assessment, 68(2), 414-435), Client Motivation for Therapy Scale with additional demographic and injury related questions was adapted for a physical therapy environment. The questionnaire was distributed to patients around the midpoint of their prescribed therapy plan at two area physical therapy clinics. Descriptive and chi-square statistics were used. Results showed that $59 \%$ of respondents were competing in sport; $61 \%$ for over 10 years. $66 \%$ reported seeking therapy for an acute injury of which $59 \%$ were injured playing a sport; $15 \%$-home accident; $13 \%$-recreational activity; $7 \%$-car accident. The most common injury was a sprain (41\%) followed by strain (28\%). The patients had participated in therapy on average $3.54 \pm 1.56$ times before the current injury. On the self-determination motivation continuum, the subscale means were: Intrinsic Motivation (4.32 \pm 0.107); Identified Regulation (4.13 \pm 0.30); Integrated Regulation (4.06 \pm 0.21 ); External Regulation (3.52 \pm 0.53 ); Introjected Regulation ( $2.77 \pm 1.35)$; Amotivation (1.79 \pm 0.96) (mean $\pm \mathrm{s}$ ). The top two individual motivators were "It is important to remain in therapy until it is finished" (introjected) and "The pleasure and satisfaction when I learn new things about myself that I didn't know before" (intrinsic). No significant differences $(P>.05)$ in responses were found between non-athletes and athletes, gender, or type of injury. Measures like these can help to identify those that are on the more external locus on the selfdetermination continuum and strategies can be developed to help patients move along the continuum to more integrated regulation and intrinsic motivation. Importantly, more autonomous motivation is associated with sustained engagement in the desired behavior, in this case, adherence to therapy (Vansteenkiste, et al., 2004, Journal of Personality and Social Psychology, 87(2), 246-260).

\section{D1.P23. Sport mindset and attitude toward dieting among kinesiology students}

\section{BORIS BALENT ${ }^{*}$, KSENIJA BOSNAR ${ }^{2}$, SARA PROT $^{3}$ \& TAMARA CVIJANOVIC ${ }^{4}$ \\ ${ }^{1}$ Croatian Association of Sport Psychologists, ${ }^{2}$ Faculty of Kinesiology, University of Zagreb, ${ }^{3}$ Coventry University, \\ ${ }^{4}$ National Ice Centre \\ *Corresponding author: boris.balent@gmail.com}

Researching core beliefs about oneself, Carol Dweck developed mindset concept that explains success or failure attributed to abilities or effort (Dweck, 1999, Self-theories: Their role in motivation, personality and development. Philadelphia: Psychology Press). According to those attributions she developed the concept of fixed and growth mindset. The aim of this research was to establish the relationships of sport mindset and the attitude toward dieting. As part of doctoral study the research received institutional approval by the Ethics Committee of the Faculty of the Kinesiology, University of Zagreb. The research was conducted on the sample of 242 students of kinesiology from The University of Zagreb, Croatia. More than half, $61,97 \%$ are male and $38.03 \%$ are female students; the age of the subjects ranges age 19 to 27; the mean age in the sample is 21,6 years. The students were measured anonymously, in groups during regular lectures. The students were given Sport mindset scale (SMS, Balent, \& Bosnar, 2014, 7th international scientific conference on kinesiology proceedings book, 7, 490-494), the scale of assessments of proportions of effort and abilities in the sport success expressed as percentages (Dweck, 2006, Mindset: The new psychology of success. New York: Random House), and Attitude toward dieting scale (ADS, Bosnar, \& AmbrosiRandić, 2004, Psiholog i zajednica. Sažeci radova 12. godišnje konferencije hrvatskih psihologa, 12, 93). The total results of SMS and ADS were determined as the result on the first principal component of the item correlation matrix. The product-moment correlations of variables were computed; as expected, SMS and percentages given for effort and abilities have low, but statistically significant correlations $(0,25$ and $-0,25, P<0.05)$. Sport mindset scale and ADS also have a low statistically significant correlation $(0,26, P<$ $0.05)$; unexpectedly, a tendency to fixed mindset is connected to more positive attitudes toward dieting. We can give few explanations: (1) the specificity of our sample, (2) explicit measure of mindset which is prone to socially desirable responding; (3) mindset might differ in different environment, so some people might have one type of mindset concerning achievement in sport, and other type of mindset in relation to dieting. The future research should be conducted on different samples and use the implicit measure of mindset. 
D1.P24. Preparing talented and elite gymnasts for within-career challenges

\section{SUZAN J.E. BLIJLEVENS ${ }^{1,2^{*}}$, PAUL WYLLEMAN ${ }^{1,2}$, MARIJE} T. ELFERINK-GEMSER ${ }^{3}$, CHRIS VISSCHER ${ }^{3} \&$ KAYAN BOOL ${ }^{2}$

\author{
${ }^{1}$ Vrije Universiteit, ${ }^{2} N O C^{*} N S F,{ }^{3}$ Rijksuniversiteit \\ Groningen \\ *Corresponding author: suzan.blijlevens@vub.ac.be \\ @suzanblij
}

According to the stage-environment fit theory there should be a fit between individual characteristics and the environment and that this fit change as individuals go through different stages of development (Eccles, \& Midgley, 1989, Research on motivation in education, 3, 139-186). Considerable research shows the role of psychological factors as determinants of individual characteristics for elite performance. However, little is known about the psychological competencies that contribute to successful talent development. Therefore, the purpose of the present study is to determine the psychological competencies (i.e., knowledge, skills, attitude, experience) talented and elite female gymnasts possess in different stages of their athletic development. With institutional ethics approval, indepth interviews were used to explore the behaviours and psychological competencies gymnasts exhibited during training, competition and daily life to deal with the challenges of their athletic career. Sixteen talented and Olympic gymnasts ( $M$ $=16.5$ years, $\mathrm{SD}=4.6$ years) participated in the study. Participants were divided into three groups, according to the stage of their athletic development: initiation, development and mastery stage (Wylleman \& Rosier, 2016, In Raab, Wylleman, Seiler, Elbe, \& Hatzigeorgiadis (Eds.), Sport and exercise psychology research: From theory to practice (pp. 270-288). Oxford: Elsevier Inc.). Thematic data-analysis was used to draw relevant themes and categories from the data. Fifteen psychological competencies were identified from the interviews: attentional focus, adaptability, balancing sport, school and social activities, competitiveness, decision-making, effective communication, goal-setting, performance under pressure, perseverance, planning, problem-solving, protecting one's boundaries, reflecting, self-confidence and thinking and acting in processes. Gymnasts in all stages of development reported behaviours related to those psychological competencies, except the gymnasts in the initiation stage; they did not report behaviours of planning and competitiveness. Further, consequently to the changes in the challenges, gymnasts are confronted with during different stages of their athletic career, the extent to which they use those competencies varied during the athletic career. Considering that the effectiveness of coaching and support of talented athletes may be diminished if they are not appropriate to the developmental stage of the athlete, our study provides useful insights for those working with talented and elite athletes to give appropriate support in developing the psychological characteristics needed at the highest level of performance.

D1.P25. The effects of motor imagery on tennis serve performance

MARIETTE BOAL* \& ITAY BASEVITCH

Anglia Ruskin University
*Corresponding author:

mariette.boal@student.anglia.ac.uk

Motor imagery contributes to enhanced motor performance; refocus, familiarisation, motivation, self-confidence, success and perfecting skills are all advantages of motor imagery use (Amasiatu, 2013, Educational Research International, 1, 69-77). However, less is known on methods of training motor imagery to improve performance. The present study aimed to examine the effectiveness of a short training imagery intervention, using the Movement Imagery Questionnaire-Revised 3 (Hall \& Martin, 1997, Journal of Mental Imagery, 21, 143-154). The questionnaire was used to train participant's ability to image four movements using internal, external and kinaesthetic imagery. After institutional ethics approval, thirty male and female tennis players (mean age: $42.9 \pm 16.3$ years; experience $14.2 \pm 15.5$ ) were recruited to participate in the study. Participants completed a baseline test of ten tennis serves to a target area, with accuracy and speed (mph) being recorded. Participants were then split into three groups of ten; 20 min training imagery group, non-training imagery group (but were instructed to use imagery during the post-test) and a control group (not training and no instructions to use imagery). Although the non-training imagery group and control group did not receive treatment, they viewed a video related to tennis that was about $20 \mathrm{~min}$ in duration (similar in duration as intervention for the training group). A post-test, identical to the baseline, was then completed, including performing ten tennis serves to a target area. Results indicated that there were no significant differences among the groups for serve accuracy, $F(2,27)=.20, P=.82, \eta^{2}=.015$, and for speed $F$ $(2,27)=.12, P=.89, \eta^{2}=.009$. Although no significant differences were found, mean accuracy and speed descriptively improved post intervention for the training imagery group compared to the non-training and control groups. Previous research examining the effects of motor imagery on performance found significant improvements. However, previous studies used longer motor imagery training sessions, whereas the current study used a short intervention. Importantly, this study also examined the effects of instructing to use motor imagery (without training), which to the best of our knowledge hasn't been examined before. Future studies should examine the effects of additional motor imagery intervention methods (e.g., modelling, feedback assisted) and durations on similar sport specific motor tasks.

\section{D1.P26. Effects of exercise intensity on} anticipation timing performance during a cycling task in children aged 7-10 years

RUTH BOAT ${ }^{1 *}$, MARTYN MORRIS ${ }^{2}$, LEANNE RAYMOND ${ }^{2}$, JOSH HURST ${ }^{2}$, JASON TALLIS ${ }^{2}$, EMMA EYRE ${ }^{2}$, VICTORIA JONES $^{2}$, CHELSEY LAWSON $^{2} \&$ MICHAEL DUNCAN ${ }^{2}$

\section{${ }^{1}$ Nottingham Trent University, ${ }^{2}$ Coventry University \\ *Corresponding author: ruth.boat@ntu.ac.uk}

The effect of exercise on cognitive performance has been extensively researched; however, conclusions appear to be equivocal (Lambourne et al., 2010, Brain Research, 1341, 12-24). The majority of studies have explored cognitive 
performance on exercise cessation rather than during exercise (Lambourne et al., 2010). In addition to this, few studies have empirically explored the effect of exercise intensity on cognitive performance in children. Therefore, this study examined coincidence anticipation timing (CAT) performance at moderate and fast stimulus speeds before, during, and after a 15 min cycling task. Following institutional ethics approval and informed parental consent, 15 children (11 males, 4 females) aged 7-10 years (mean age \pm SD $=8.2 \pm$ 1.3 years) took part in this study. Exercise was performed on a cycle ergometer (Corival Pediatric, Lode B.V., Netherlands) under two experimental conditions: exercise intensities of $50 \%$ and $75 \%$ heart rate reserve (HRR). Coincidence anticipation was measured using the Bassin Anticipation Timer (Model 35575, Lafayette, USA) at speeds of 5 and $8 \mathrm{mph}$. Repeated measures ANOVA's were then conducted to evaluate the effect of exercise intensity on coincidence anticipation performance before, during, and immediately after the cycling task. Results indicated that for absolute error (AE) there was no significant main effect for time $(P=.818)$ or condition $(P=.452)$, or interaction effect between experimental condition and time $(P=.096)$ at the $5 \mathrm{mph}$ stimulus speed. Similar results were also found at the $8 \mathrm{mph}$ stimulus speed; there was no significant main effect for time ( $P=$ $.915)$ or condition $(P=.958)$, or interaction effect between experimental condition and time $(P=.483)$. This study suggests that exercise intensity has no effect on anticipation timing during a cycling task in paediatric populations. However, replication studies, with larger sample sizes, are required to confirm our findings. Overall, exercise may facilitate decision making and complex problem solving (Tomporowski, 2003, Acta Psychologica, 112, 297-324), but this study demonstrates that exercise does not appear to effect visual information processing in children.

D1.P27. An interdisciplinary approach to problem solving: a case study of a professional football academy

\section{CHRIS BRADLEY*}

\section{Middlesbrough Football Club \\ *Corresponding author: chris.bradley@mfc.co.uk}

Like many areas of high performance sport, the academy football environment is dynamic and at times volatile. As a Sport Science and Medicine department it is important to adopt a proactive, flexible, solution-focused approach to our work. This is made possible by a cycle comprising regular knowledge-sharing opportunities, collaboration in applied work, and multidisciplinary reflective practice. The aim of this presentation is to communicate the role sport psychology support plays within a multidisciplinary team to solve complex problems in a professional football academy context. The nature of typical problems will be described, while a model illustrating the problem solving and prevention process will be presented and critically discussed. The experience of practitioners will be translated into methods and processes that the audience can adapt and apply in their sporting context. A traditional, triangulated approach is taken to needs analysis. Information pertaining to athlete support is acquired and disseminated through observation, formal and informal discussions, and testing. From a psychological perspective, practitioners work in a closely integrated manner, while carrying out clearly defined roles. One works with players aged 9-16 and part-time coaches, while the other works with U18 and U23 squads and lead coaches. The programme revolves around three areas: performance, life skills, and mental health. For players in the foundation phase, the aim of the programme is to educate players around sport psychology concepts and skills. In the Youth Development Phase, there is a shift in focus to emotional support, leadership, and performance-specific strategies. Finally, in the professional phase, performance support becomes more bespoke to each individual and players are encouraged to consider career pathways both within and outside of football. There is a mental health assessment and referral programme in place for all players to access where necessary and appropriate. Several methods of assessment are used to assess players and subsequently the effectiveness of the psychology programme. Mental state examinations are used as screening tools for potential referrals to appropriate services. Performance profiling is used to quantitatively track player's psychological changes across a season. Performance Analysis is used to capture video footage of player and coach behaviours in training and games. Time constraints are always a factor in high performance sport and the Academy football environment is no different. There are around 150 players in the academy, making it difficult or impossible for a psychologist to see all these players individually. Group workshops are useful from an educational perspective but their applied impact is limited. This session will critically evaluate the successes and areas to improve of a constantly evolving programme.

\section{D1.P28. The effects of media feedback on soccer players' motivation across gender}

\section{PEDRO BRUNO \& ITAY BASEVITCH}

\section{Anglia Ruskin University \\ *Corresponding author: pmpb6128@gmail.com}

Soccer is one of the most popular and watched sports around the world (Halvari et al., 2012, Journal of Medicine and Science in Sports, 22, 569-579). Previous studies have indicated that media coverage can affect motivation, emotions and performance (Carpentier \& Mageau, 2013, Psychology of Sport and Exercise, 14, 423-435). At university soccer, the exposure is mainly from local media entities that reports what happens during British Universities and College Sports (BUCS) championship. This type of exposure could produce some impact into students' motivation and performance, considering the majority might not be psychologically prepared to cope with this type of pressure. Furthermore, gender stereotypes are evident in media coverage of sport (El-Alayli \& Hively, 2014, Psychology of Sport and Exercise, 15, 48-55). However, most studies have focused on professional and high-level players. Thus, there is a gap in the literature in understanding the effects of exposure of university level athletes' to media feedback on motivation. The purpose of the study was to examine the relationship between media feedback and athletes' motivation across gender. Following institutional ethics approval, soccer 
players ( $n=80,40$ males and 40 females) were recruited from different Universities and Colleges in the Cambridgeshire county, United Kingdom. The Sport Motivation Scale (SMS-28; Blais et al., 1995, Journal of Sport and Exercise Psychology, 17, 35-53) was used to measure players' motivation to play soccer pre and post media feedback. Participants were divided to two groups of media feedback, where group 1 received positive feedback and group 2 received negative feedback. Results from the RM ANOVA suggested a significant decrease in intrinsic $(P=.08$, $\left.\eta^{2}=.09\right)$ and extrinsic motivation $\left(P=.04, \eta^{2}=.10\right)$ and an increase of amotivation levels $\left(P<.01, \eta^{2}=.22\right)$ when receiving negative feedback from the media. Regarding positive feedback, findings showed a significant increase in intrinsic $\left(P<.01, \eta^{2}=.29\right)$ and extrinsic motivation $\left(P<.01, \eta^{2}=.14\right)$ and a decrease of amotivation levels $\left(P<.01, \eta^{2}=.29\right)$. Furthermore, there were significant differences between the genders; females were less affected by the feedback related to intrinsic motivation $\left(P<.01, \eta^{2}=.08\right)$ and amotivation $\left(P<.01, \eta^{2}=.12\right)$, but no differences were found in extrinsic motivation $\left(P=.13, \eta^{2}=.03\right)$. Findings indicate that media feedback affects motivation levels of University level athletes and that males are more sensitive to media feedback. Further studies should examine the different effects media outlets (e.g. articles, social media, television) from well-known media sources, have on athletes' motivation, at various levels (e.g. youth, university and elite level).

\section{D1.P29. A cross cultural analysis of the management policies and the motives of volunteers within Special Olympics}

\section{LIZ CARLIN* \& DAVID HASSAN}

\section{Ulster University \\ *Corresponding author: carlin-14@ulster.ac.uk @lizcarlin6}

Sports organisations have traditionally relied heavily on the work of volunteers, yet research suggests that many organisations struggle to recruit adequate numbers with the sports coaching role being notoriously difficult to recruit and retain. In order to assist the recruitment and retention process, it is important for volunteers to feel connected with the values of the organisation and also that they have an input into the decision-making processes within the organisation. The overarching aim of this paper is to gain an understanding of the volunteers' views on the processes and structures of volunteer management and retention within Special Olympics. Conducting this research in three European countries, with a combination of countries which has and has not hosted an International Special Olympics event: European or World Games, will provide a greater understanding of the different policies adopted by the National Programs as well as outlining any cultural differences in the motives and opinions of the volunteers themselves. An online questionnaire was issued to volunteers within each of the three national programs receiving 409 respondents. Using the Coach Motivation Questionnaire (McLean, Mallett \& Newcombe, 2012, Journal of Sport \& Exercise Psychology, 34, 184-207) volunteers within Special Olympics scored significantly higher on Intrinsic Motivation questions than on questions relating to Amotivation on the Self Determination Theory Continuum (Deci \& Ryan, 2002, Handbook of self-determination research. Rochester, NY: University of Rochester Press). Additionally, across all countries, Special Olympics volunteers scored higher on all elements of the CMQ than the CMQ average rating except for the amotivation scale which was lower than average. The response rate per country provides significant insight into volunteer engagement with 203 responses (5.05\%) from Special Olympics Ireland, 150 responses (3.2\%) Special Olympics Hellas and 56 responses (1.2\%) Special Olympics Great Britain. Volunteers generally were unaware of the policies and procedures adopted by their National Programs, however there was a general consensus that recruitment is primarily ad hoc and should be more strategic. Special Olympics Ireland volunteers wanted to be more involved in decision making processes within the organisation with Special Olympics Hellas volunteers more likely to state that they did not have the knowledge to do this. Overall, a more co-ordinated response is required from National Programs in relation to the recruitment and retention of volunteers and this response needs to be tailored to the specific demographics of the volunteers within each National Program. Ethical approval for this research was obtained from the Ulster University ethical committee.

\section{D1.P30. Development of a psychological development programme to support youth professional footballers in a Premier League football academy}

\section{LEWIS CHARNOCK ${ }^{1,2 *}$, MARTIN LITTLEWOOD ${ }^{2}$, ROB MORRIS $^{2} \&$ MARK NESTI ${ }^{2}$ \\ ${ }^{1}$ Everton Football Club Academy, ${ }^{2}$ Liverpool John Moores University \\ *Corresponding author: L.Charnock@2016.ljmu.ac.uk}

The Professional Footballers' Association (PFA, 2016) estimates that out of an academy scholarship U18 squad, typically consisting of 16 players, only one player will remain playing at a professional level by the age of 21. Despite research presenting aspects such as self-awareness, resilience and environmental factors as fundamental facets which negatively or positively influence the development of elite youth academy football players (Morris et al., 2016, International Journal of Sport and Exercise Psychology, 1, 1-17), there is still a lack of understanding of how football academies can help foster and develop these attributes to positively influence player development. Organisational culture is an emerging topic in sport psychology and recent literature (Larsen et al., 2016, Sport, Exercise, and Performance Psychology, 2, 190-206; Henriksen, 2015, Journal of Sport Psychology in Action, 6, 141-153) has argued that creating and maintaining high-performance cultures is important with regards to environmental success factors in talent development. With institutional ethics approval, building on the early work (Parker, 1995, PhD thesis, University of Warwick), the present study is an ethnographic account from a researcher-practitioner embedded within the culture of an elite Premier League football academy with understanding of the inner circle and working practices of the 
organisation. The present study aims to examine the culture and talent development environment among youth male footballers in an English Premier League football academy with a heritage of successfully developing juniors to toplevel football players. Principal methods of data collection include interviews, participant observation, field notes and questionnaires. The environment was characterised by a highly pressurised climate for success and desperation to progress to the senior level of professional football. Furthermore, the volatile cultures at academy and first team levels are such that youth players encounter critical moments several times during their academy journey. Results are presented from various stakeholders (parents, players, coaches and staff) with regards to the existing culture and how it may impact successful talent development. It is argued that the current findings may hold the potential to influence key stakeholders to be sensitive not only to individual player needs, but also the overall organisational culture and daily interaction in the talent development environment. The current case study is the first part of a wider body of work that the researcher is conducting with a view to identifying issues that contribute or prevent successful within-career transitions of youth footballers to make the grade in first team football and beyond.

\section{D1.P31. Exploring athletes' perceptions of group flow in team performance}

\section{ADAM COUSSENS* \& LEWIS KING}

\section{University of Lincoln \\ *Corresponding author: acoussens@lincoln.ac.uk @adamcuzz}

Despite extensive research exploring the psychological phenomenon of flow in performance contexts for individuals, there is a paucity in literature with regards to the experience of group flow in team sports. Whilst group flow has received attention in predominantly non-sporting domains, only a limited amount of research has explicitly explored group flow experiences of athletes (Bakker et al., 2011, Psychology of Sport and Exercise, 12, 442-450). As such, the aim of this study was to investigate perceptions of group flow experience of athletes that competed in team sports, applying a qualitative framework. A particular emphasis was placed on exploring the characteristics involved in group flow and the factors that facilitate or disrupt this experience. With institutional ethical approval, semi-structured interviews were conducted with eight $(N=8)$ athletes (mean age: $24.0 \pm 3.96$, years' experience playing their sport $9.9 \pm 2.17$ ) from a variety of standards (ranging from national to recreational), and sports (including soccer, rugby, tennis doubles, camogie, and handball). The trustworthiness of the data was established through peer debriefs, critical peer review, and member checking. Subsequent data analysis included both inductive and deductive analysis, based on Sparkes and Smith's (2013, Qualitative research methods in sport, exercise and health: From process to product. London: Routledge) recommendations. Inductive analysis created higher order themes from the raw data, and these themes were deductively coded into Csikszentmihalyi's (2002, Flow: The classic work on how to achieve happiness. 2nd ed. London. Rider
Books) model of flow, producing eight characteristics describing the experience of group flow. When athletes experienced group flow they also perceived an enhancement in team performance, and shared understanding with teammates. In particular, a perceived shared understanding between teammates was associated with heightened automaticity of movement and decisions. Group flow was reported to be facilitated by high levels of confidence within the team at both an individual and collective level, and by positive team play and interactions. Group flow disruptors included negative interactions from team-mates, such as criticism. The current study provides a detailed understanding of athletes' perceptions of group flow in relation to current flow theories, with considerations outlined for theoretical development and practical application of developing group flow.

\section{D1.P32. The importance of life skills for sports} degree students: how they contribute to students' academic performance, health-related quality of life and flourishing

\section{LORCAN CRONIN $^{1 *}$, JUSTINE ALLEN ${ }^{2}$, PAUL ELLISON ${ }^{1}$, DAVID MARCHANT' ${ }^{1}$, ANDREW LEVY $^{1} \&$ CHRIS HARWOOD $^{3}$}

\section{${ }^{1}$ Edge Hill University, ${ }^{2}$ University of Stirling, \\ ${ }^{3}$ Loughborough University \\ *Corresponding author: Lorcan.Cronin@edgehill.ac.uk}

Life skills are defined as a range of transferrable skills needed for everyday life. According to Benson and Saito's (2001, In Benson \& Pittman (Eds.), Trends in youth development: Visions, realities and challenges (pp. 135-154). London: Kluwer Academic Publishers) framework for youth development theory and research, life skills impact upon young people's academic performance, health and well-being. Thus, the aim of this study was to explore the relationships between university sports degree students' life skills (teamwork, goal setting, time management, emotional skills, interpersonal communication, social skills, leadership, and problem solving) and their academic self-efficacy, predicted academic performance, health-related quality of life (physical, emotional, social, and work/school functioning), and flourishing. With institutional ethics approval, 423 sports degree students (male $=236$, female $=187$, mean age $=$ $20.42 \pm 2.56$ years) completed a survey assessing the main study variables. This survey used existing measures to assess all variables except for life skills, which were assessed using a newly developed scale. Standard multiple regression analyses revealed that time management $(B=.51, P<.001)$, problem solving $(B=.23, P<.01)$, and goal setting $(B=.15, P$ $<.05)$ contributed significantly to students' academic selfefficacy. Time management was the only significant contributor to students' predicted academic performance $(B=$ 2.23, $P<.001)$. In terms of health-related quality of life, emotional skills $(B=.23, P<.001)$ were the only significant contributor to students' emotional functioning, social skills ( $B=.18, P<.001$ ) were the only contributor to students' social functioning, and both time management $(B=.25, P<$ $.001)$ and problem solving $(B=.14, P<.05)$ contributed significantly to students' work/school functioning. Finally, 
five life skills contributed significantly to students' flourishing: social skills $(B=.27, P<.001)$, leadership $(B=.26, P<$ $.01)$, emotional skills $(B=.20, P<.001)$, goal setting $(B=.14$, $P<.01)$, and time management $(B=.13, P<.01)$. Interpretation of the results suggest that university degree programmes and staff (e.g., lecturers or learning services) should help sports degree students develop these life skills which contribute to their academic performance, healthrelated quality of life, and flourishing. In practice, staff could provide specific learning sessions during the degree programme, that target the development of key life skills at what is a critical developmental period of young adulthood.

\section{D1.P33. Investigating the factor structure of the Dual Career Competency Questionnaire for Athletes in European student-athletes}

\section{KOEN DE BRANDT ${ }^{1 *}$, PAUL WYLLEMAN ${ }^{1} \&$ MIQUEL TORREGROSSA ${ }^{2}$}

\section{${ }^{1}$ Vrije Universiteit Brussel, ${ }^{2}$ Universitat Autónoma de Barcelona \\ *Corresponding author: koen.de.brandt@vub.be @KoenDeBrandt}

Qualitative research identified a range of important dual career (DC) competencies that facilitate athletes' progression in their dual "study and elite sport" career pathway (e.g. MacNamara \& Collins, 2010, Psychology of Sport and Exercise, 353-362). However, a valid and reliable instrument that measures athletes' important DC competencies was lacking. In response to this lack in methodology, the Dual Career Competency Questionnaire for Athletes (DCCQ-A) was developed and used as part of the Erasmus+ Sport project "Gold in Education and Elite Sport". The specific aims of this study were to (a) investigate the latent factor structure of the DCCQ-A, and (b) examine athletes' perceptions of the importance and possession of the factors identified in the DCCQ-A. With institutional ethics approval, 3,350 15-to-26-year-old athletes (mean age: $18.6 \pm 2.5 ; 53 \%$ male; $76 \%$ pupils and $24 \%$ students) from nine European countries completed the DCCQ-A in which they rated their perceived importance and possession of 38 DC competency items (i.e., DC knowledge, skills and attitudes) on a 5-point Likert-type scale. Exploratory Structural Equation Modelling (ESEM) was used to investigate the factor structure of the DCCQ-A. Athletes' perceived importance and possession of DC competencies were analysed using descriptive statistics in SPSS. ESEM supported a 29-item four-factor structure of the DCCQ with satisfactory psychometric properties (RMSEA $=.049, \mathrm{CFI}$ $=.952, \mathrm{TLI}=.934)$ and adequate internal consistency scores ( $a=.75-87$ ). The factors reflected four DC competencies: (a) DC management (e.g. time management, planning), (b) Career Planning (e.g. preparing for the unexpected, career exploration), (c) Mental Toughness (e.g. stress management, resilience), and (d) Social Intelligence \& Adaptability (e.g. making and maintaining social contacts). Furthermore, athletes perceived all four DC competencies as important for a successful DC (mean: 4.15-4.40) and reported average to good possession of the four DC competencies (mean: 3.523.85). The study advances previous research by providing the first measure of athletes' DC competency, and suggests the four-factor structure of the DCCQA-29 to be used as an evidence-based framework for DC practitioners to operationalize their work with student-athletes.

D1.P34. Ethical guidelines for dual career stakeholders: a focus group study with European dual career experts

\section{SIMON DEFRUYT ${ }^{1 *}$, PAUL WYLLEMAN ${ }^{1}$, MIQUEL TORREGROSSA ${ }^{2}$, NATALIA STAMBULOVA ${ }^{3} \&$ NICOLETTE SCHIPPER-VAN VELDHOVEN ${ }^{4}$}

\section{${ }^{1}$ Vrije Universiteit Brussel, ${ }^{2}$ Universitat Autónoma de Barcelona, ${ }^{3}$ Halmstad University, ${ }^{4}$ Windesheim University of Applied Sciences \\ *Corresponding author: simon.defruyt@vub.be @SimonDefruyt}

A growing number of elite athletes is combining elite sport and education (i.e. is pursuing a dual career). Governmental institutions, elite sport organisations and educational institutions (need to) support this combination to avoid that athletes are forced to choose between one of two endeavours. Although the EU Guidelines on Dual Careers of Athletes (2012) formulate valuable recommendations on how this support should be organised on a policy and organisational level, specific dual career (DC) guidelines on a micro level are lacking. Therefor the aim of this qualitative study is to formulate expert-based ethical guidelines for DC stakeholders. With the acquired ethical approval, 12 participants from 4 European countries are involved in a focus group, organized within the 'Advanced Olympic Research Grant Programme'. The 12 participants consist of experts in dual career, both from the academic and practice field. A semistructured approach is adopted within the focus group discussion. After transcribing the focus groups making use of Otranscribe, thematic analyses are conducted using Nvivo 10.2. Qualitative findings reveal emerging guidelines grouped into 4 main clusters: (1) the professional relationship with DC athletes, (2) confidentiality, (3) lifelong learning, (4) cooperation with other DC stakeholders. The findings are specific to the European DC context, but general with regard to (1) national context (i.e. a European perspective), (2) the specific type of organisation (e.g. governmental organisations, sport organisations, educational institutions), (3) the type of DC stakeholders (e.g. coach, teacher, study counsellor), (4) type of (challenging) situations for DC stakeholders. Practitioners can use current findings to develop codes of conduct and other guiding documents for DC stakeholders. However, researchers and practitioners are encouraged to tailor and contextualize current results towards the variables mentioned above (i.e. national context, type of organisation, type of DC stakeholders, specific challenging situations). Within the 'Advanced Olympic Research Grant Programme', the results will be used to develop an educational module for dual career support providers. Future research could pilot test this educational module with a sample of dual career support providers, further exploring and testing the application of the ethical guidelines for DC stakeholders. 
D1.P35. Providing services to youth athletes: when does parental involvement become an issue?

\section{KOTRYNA FRASER ${ }^{1 *}$}

${ }^{1}$ The University of Edinburgh

*Corresponding author: kotryna.fraser@ed.ac.uk @coach_kotryna

Sport and exercise psychology practitioners provide services to a diverse range of clientele ranging in terms of experiences and abilities, age, gender or cultural background (Hanrahan, 2010, In Hanrahan \& Andersen (Eds.) Routledge handbook of applied sport psychology (pp. 460-468). Indeed, pros and cons of parental involvement in sport, and proving support to young athletes and their families have become a more recognised topic within sport and exercise psychology (Knight et al., 2017, Current Opinion in Psychology, 16, 93-97). However, unique challenges of providing services to youth athletes (i.e. 16-to-18 years of age) and parental involvement in both sport and sport psychology support services have not been extensively addressed yet. The challenges here often include athlete's age of maturity, increasing autonomy to make decisions or growing commitments for both school and sports team. This poster, therefore, aims to compare, contrast and reflect on applied work conducted with two youth athletes from different sporting environments in Scotland (i.e. motorsport and swimming), and with significantly distinct levels of parental involvement. In both cases detailed needs analyses were conducted which involved parents and coaches to better understand the athletes, their environments and individual needs. Following the process of needs analysis, case conceptualisation and proposal of evidence-based intervention, practitioner undertook two different approaches to manage parental expectations and involvement. This was due to significant differences to what extent parental involvement was reasonable and enhancing rather than inhibiting athlete's performance in both case studies. For instance, a swimmer made critical decisions in collaboration with parents whereas a driver obeyed father's authoritarian decisions (e.g., how to progress after experiencing adversity). Similarly, lack of commitment and parental perception on this issue differed significantly in both case studies. The importance of familiarising practitioners with unique challenges presented by both youth athletes and their parents is discussed in light of practitioner's experiences, self-reflections and peer-mentoring process. It is crucial to appropriately prepare practitioners-in-training to effectively deal with challenges presented by this unique age-group athletes and their parents.

\section{D1.P36. Biochemical variations and erythrocyte membrane stability after resistance training session}

CAROLINA FREITAS SILVA ${ }^{1}$, IGOR SOMBRA SILVA ${ }^{2}$, MARIO DA SILVA GARROTE FILHO ${ }^{2}$, NILSON PENHA SILVA ${ }^{1}$, MIGUEL JUNIOR SORDI BORTOLINI ${ }^{2}$, LETICIA RAMOS DE ARVELOS ${ }^{1} \&$ ROMEU PAULO MARTINS SILVA $^{1 *}$

\section{${ }^{1}$ Federal University of Acre, ${ }^{2}$ Federal University of Uberlândia \\ *Corresponding author: romeupms@gmail.com}

Introduction: The practice of physical exercise is a factor that can cause a significant increase of enzymes indicative of muscle injury and promotes hemorheological adaptations in the blood, such as changes in aggregation, deformability and fluidity of erythrocytes. Objective: To verify the effect of a single physical training session of multiple series and circuits on the enzymatic variations and the erythrocyte membrane stability. Methods: The study population consisted initially of 108 men who finished with 21 healthy and active men. Participants held single sessions of two types of training. The first session consisted of circuit training and the second session, held a week later, consisted of a multi-series training session. Blood samples were taken before and immediately after training for the determination of erythrocyte stability and for biochemical determinations. The osmotic stability of the erythrocyte membrane was represented by the inverse salt concentration $(1 / \mathrm{H} 50)$ at the midpoint of the sigmoidal curve of dependence between hemoglobin absorbance and $\mathrm{NaCl}$ concentration. Results: There were no changes in erythrocyte membrane stability index in circuit training and multiple series. When compared to the enzyme concentrations before and after the different training sessions, plasma creatine kinase (CK$\mathrm{NAC})$, creatine kinase isoenzyme (CK-MB), lactate dehydrogenase (LDH), aspartate aminotransferase and AST, Alanine Aminotransferase (ALT) were higher in post-training at $P<$ 0.05 . In the comparison between the different training sessions, only the plasma concentrations of CK-MB and CK-NAC had their highest values in the multiple series after training at $P<0.05$. The heart rate was higher after the two training sessions, which presented higher values in the training after the training, with $P<0.05$. Conclusion: A single-circuit and multiple-series training session did not affect the osmotic stability of erythrocytes, with variation in muscle enzymes leading to increased lesion in multiple training series and greater variation in cardiac capacity with higher heart rate values in circuit training.

\section{D1.P37. The relationship between young club} swimmers' perception of task and ego orientated parental feedback and motivation

\section{MAITA FURUSA \& ITAY BASEVITCH*}

\section{Anglia Ruskin University \\ *Corresponding author: itay.basevitch@anglia.ac.uk}

Several studies have examined the importance of parental influence on children's involvement and achievement in education and sporting domains (Lavoi \& Stellino, 2008, The Journal of Psychology, 142, 471-496). However, there is limited research on the affect parents have on young swimmers. The purpose of this study was to explore young club swimmers' own goal orientation (task or ego) and their perceptions of the parent-initiated motivational climate. In addition, the aim was to examine whether swimmers' perceptions of their parents' views varied as a function of 
swimmers' age, gender, and level of practice. Participants were $n=29$ junior club level swimmers ( $n=14$ girls and $n=$ 15 boys), whom competed at various levels. Participants' average age was $13.58 \pm 1.19$ years. Approval was granted from the chair of the Faculty Research Ethics Policy under the terms of Anglia Ruskin University's policy. Following the approval, participants completed the 12-item Task and Ego Orientation in Sport Questionnaire (TEOSQ; Duda \& Nicholls, 1992, Journal of Educational Psychology, 84, 290-299) and the 18-item Parent Initiated Motivational Climate Questionnaire-2 (PIMCQ-2) (White \& Duda, 1993, Adapted Physical Activity Quarterly, 10, 125-136). A Pearson correlation was conducted and revealed a significant positive correlation between swimmers' self-reported task and their perceptions of the parental task $(r(9)=0.39 P=0.04)$, and between their ego orientation and their perceptions of their parents ego initiated motivational climate $(r(9)=0.37 P$ $=0.05$ ). Furthermore, results indicated a significant negative correlation between swimmers' age and perceptions of their parents task orientation $r(9)=0.43 P=0.02$, but no significant relationship with their parents ego orientation $r(9)$ $=-0.06 P=0.97$. With regards to gender differences, $\mathrm{a}$ one-way ANOVA revealed, no significant differences between boys and girls with regards to perceived parental ego $F(1,27)=1.3, P=.26$ and task $F(1,27)=2.5, P=.11$ motivational climate. However, results showed that boys scored significantly higher in their own ego orientation $(M=3.65, S D=0.20)$ compared to girls $(M=3.01, S D=$ $0.22), F(1,27)=4.4, P=.04$ but no significant differences were found between genders on swimmers' own task orientation, $F(1,27)=1.1, P=.29$, Overall results from the current study suggest that there is a moderate positive relationship between young club swimmers perceived parental motivational climate and their own dispositional goal orientation. In addition, results suggest that young male swimmers are more ego orientated than females however, there is no gender difference in how the parental motivation climate is perceived. Therefore, the findings support the notion that parents play an important role in influencing the motivation climate of young club swimmers.

\section{D1.P38. Working on the edge: an ethnographic study of stress, coping and expedition leadership}

\section{DANNY GOLDING*, GAIL KINMAN \& STEVE KOZUB}

\section{University of Bedfordshire \\ *Corresponding author: danny.golding@beds.ac.uk}

As the depth of research focusing on stress and coping in physical activity continues to rapidly evolve; it is only relatively recently that attention has been devoted to those that coach within this domain (Fletcher \& Scott, 2010, Journal of Sport Sciences, 28(2), 127-137). While an expansion in this field of research is seen as desirable, there have been no studies which consider work related stress and coping in adventurous contexts. Using an ethnographic approach the aim of this study was to explore the coping processes relating to stress for leaders facilitating a 5 week expedition for young people aged 16-21. Following institutional ethical approval, a cognitive ethnography approach was adopted (Ball \& Ormerod, 2000, International Journal Human-
Computer Studies, 53, 147-168) based on a transactional stress and coping theoretical framework (Lazarus \& Folkman, 1984, Stress, appraisal and coping. New York: Springer). The study used observation, interviews and diaries to capture the experiences of 8 expedition leaders facilitating a summer expedition in a mountain region close to the Arctic Circle in Northern Norway. The findings indicate that the environmental demands remained relatively innocuous for most part as the leaders harmonised with the nove contextual challenges. Experience based judgements and routines for maintaining a degree of comfort in extreme conditions were symptomatic of the proactive coping position evident in attitudes and behaviours. This equilibrium was significantly disturbed when leaders were required to work at the extremes of their level of competencies as a result of the perceived pressure from both participants and the organising body. Such demands led to both negative emotional responses and proactive coping strategies. There was some interpersonal tension that emanated from the logistical and operational demands most evident at base camp when all groups were living in close proximity. However, working autonomously and purposefully in smaller satellite groups significantly reduced the perceived levels of stress. Seeking social support from allied colleagues also provided valued opportunities for sharing a range of identified concerns. A number of leaders recognised that the commitment to providing guidance and optimal experience for young participants in wilderness areas fostered proactive coping behaviours and provided a welcome distraction to potential tensions. It would seem that working in risky and dangerous wilderness environments requires considerable experience based proactive coping and although highly demanding, expedition leaders valued the opportunity to be working at the edge of their perceived comfort zones and recognised the potential for growth.

\section{D1.P39. Team cohesion and coping strategies in professional baskeball teams in Greece}

\section{VICKY GOLTSI* \& KONSTANTINOS MEGAGIANNIS}

\section{Metropolitan College Greece \\ *Corresponding author: vgoltsi@mitropolitiko.edu.gr}

In team sports the concept of team is integral in team's effectiveness and goal achievement. At the same time, team members' resilience, the sustainability in work pressure and the positive evaluation of difficulties can predict success as well as well-being and satisfaction of team members (Carron, Colman, Wheeler, \& Stevens, 2002, Journal of Sport and Exercise Psychology, 24, 168-188). Current study aimed to support previous research evidence of the relationship between team cohesion and coping avoidance behaviours as an effective and useful tool in team sport coaching strategies and behaviours. The research explored the relationship between the two constructs, after receiving full ethical clearance from the Research Ethics Committee of the corresponding institution. 137 professional basketball players, from the top national Championship divisions (A1 \& A2) in Greece, were recruited. Team cohesion was measured with the Group Environment Questionnaire (Carron, Widmeyer, \& 
Brawley, 1985, Journal of Sport Psychology, 7, 244-266) as it was adapted to Greek language by Aggelonidis (1995, Unpublished PhD Thesis, University of Athens). Coping Avoidance behaviours were assessed with COPE (Carver, Scheier, \& Weintraub, 1989, Journal of Personality and Social Psychology, 56, 267-283), in its adaptation to Greek population by Chatzigeorgiadis and Chroni (2003, Inquires in Physical Education and Sport, 1, 19-26). Results showed a significant positive relationship between increased levels of team cohesion and positive coping of stressful events by team's members $(r(137)=.35, P<.001)$. Similarly, team cohesion significantly predicted coping - avoidance behaviour $(\beta=.35, P<.001$ ). Current findings agree with previous ones on the role of team cohesion on coping strategies that athletes and other team members adopt in order to overcome the pressure that a demanding championship imposes. Moreover, current findings implicate that professional basketball teams in Greece could get benefited from team building and team cohesion strategies. Coaching and managing staff could support and enrich team building and the subsequent team cohesion. As such, strategies like re-building new teams from the scratch every season seem that does not help identification with the team, team climate and cohesion and as such, coping might be uncertain in times of big pressure and great demand.

\section{D1.P40. Psychosocial factors facilitating use of cognitive enhancing drugs in education: a qualitative investigation of moral disengagement and associated processes}

\section{ANDREW HEYES* \& IAN BOARDLEY}

\section{University of Birmingham \\ *Corresponding author: andrewrheyes@gmail.com @andrewrheyes}

Competition and the drive for excellence has led some individuals in sport to seek methods to enhance their performance through illicit means. Although ongoing research seeks to increase understanding in a sport and exercise context, there is limited literature exploring the use of drugs by students to increase cognitive performance. This study investigated psychosocial processes associated with the use of cognitive enhancing (CE) drugs in university students through the framework of Bandura's theory of moral thought and action (1991). In depth semi-structured interviews were conducted with 9 students from a UK university with experience of prescription drug use for the purpose of cognitive enhancement in education. The study received ethical approval from the University of Birmingham. The resulting data was content analysed deductively using definitions for the eight mechanisms of moral disengagement (Bandura, 1991). The analysis evidenced six mechanisms of moral disengagement (i.e. moral justification, euphemistic labelling, advantageous comparison, displacement of responsibility, diffusion of responsibility, and distortion of consequences). Emergent themes were discussed with reference to Bandura's theory in addition to other relevant literature on CE drug use in education and performance enhancing drug use in sport and exercise. Overall, findings suggest that when explaining their CE drugs use, students would frequently morally disengage and the mechanisms of moral disengagement may help students to avoid morality and health-based deterrents when attempting to cognitively enhance. The findings demonstrate similar mechanisms of moral disengagement to those evidenced by athletes with experience of performance enhancing drug use.

\section{D1.P41. The role of sport psychology in helping athletes deal with increased media exposure}

\section{TANJA KAJTNA*}

\section{University of Ljubljana}

*Corresponding author: tanja.kajtna@fsp.uni-lj.si

An inevitable part of athletic success is increased exposure to the media. Athletes learn to deal with media as they gradually gain more experience with them and they often learn from their mistakes and slowly become better at dealing with media. Good public image of an athlete not only helps gaining funding for athlete's expenses, but also helps enhance their self-image and strengthen their identity. Psychologists and athletes frequently discuss issues related to their public image and can help them prepare for interactions with media, systematic preparation for interactions with media can reduce the stress of dealing with the media. This is even more important for very successful athletes, as they interact with media more frequently. Psychologists should emphasize the importance of good psychological preparation, as it helps to keep the athlete focused on the concrete task instead of on media attention. When athletes become famous, even "stars", the issues regarding media become more evident and psychologists have an important part in helping the athletes separate their own goals and expectations from the goals the media and public sets out for them. The aim of this study was to find out how famous athletes perceive their relationship with the media, more specifically we wanted to see how media attention influenced their career, if they see the media as help or as an obstacle... We also wanted to see how they got used to the media exposure and the role of the sport psychologist in helping them deal with it. Upon ethical approval of Faculty of sport, we conducted in-depth interviews with 5 Slovenian athletes, whom we could call stars in their sport. The interviews were semi-structured and were conducted individually and recorded. Afterwards we made transcripts of the interviews and we used content analysis to compare the results of the interviews. In their answers, the athletes describe who and how helped them when dealing with the media, they describe tactical approaches on how to give answers, they describe rehearsing interviews, having an agreement with the media on when to give statements... Their answers reflect a great complexity of their "relationship" with the media and reveal great importance of discussing these issues with a sport psychologist. Psychological interventions in sport should also include topics regarding communication and we also believe that psychologists should be more in contact with media experts and journalists in educating them how to interact with athletes. 
D1.P42. The influence of self-regulated learning and coping styles on psychological resilience in sports

\section{JOLAN KEGELAERS*, PAUL WYLLEMAN \& MART TAS}

\section{Vrije Universiteit Brussel \\ *Corresponding author: jolan.kegelaers@vub.ac.be @TopsportVUB}

As it is widely recognised that the ability to deal with and effectively overcome stressful events is essential for success in sports, the field of psychological resilience has gradually gained interest in sport psychology over the last decade. Some authors have suggested that resilience can be influenced by metacognitive skills (Fletcher \& Sarkar, 2012, Psychology of Sport and Exercise, 13, 669-678), including planning, goal-setting, monitoring, and reflecting on one's own thoughts and behaviours (also referred to as self-regulated learning). The present study aimed at examining how athletes' self-regulating skills and subsequent coping strategies can act as protective factors against the possible detrimental effects of exposure to stressful events. Following institutional ethical approval, an online survey was conducted with 235 athletes $\left(M_{\text {age }}=22.84\right.$; SD $\left.=4.47\right)$. The participants completed the Self-Regulated Learning-Self Report Scale (Toering, Elferink-Gemser, Jonker, van Heuvelen, \& Visscher, 2012, International Journal of Sport and Exercise Psychology, 10(1), 24-38), the Coping Function Questionnaire (Kowalski \& Crocker, 2001, Journal of Sport \& Exercise Psychology, 23, 136-155) and the Brief Resilience Scale (Smith et al., 2008, International Journal of Behavioral Medicine, 15, 194-200). Using hierarchical regression, we found that psychological resilience could only be partially explained by self-efficacy $(16,6 \%)$, gender $(8,6 \%)$, and avoidance coping $(5,2 \%)$. Contrary to our hypotheses, specific metacognitive skills (e.g., goal-setting, monitoring, evaluation, reflection) provided no significant predictor for psychological resilience. Our study suggests that a positive response to stressful events is not necessarily the result of the specific metacognitive or behavioural strategies used by the athlete, but rather results from the inherent belief that he/she has the ability to deal with the situation.

\section{D1.P43. Autonomy support, controlling behaviours and other forms of social influences from significant others in youth sport}

\section{ALFRED SING YEUNG LEE ${ }^{1} \&$ DERWIN KING CHUNG CHAN $^{1,2}$ \\ ${ }^{1}$ University of Hong Kong, ${ }^{2}$ Curtin University \\ *Corresponding author: Isyintic@hku.hk}

Significant others, such as coaches, parents, and peers, play a crucial role in young athletes' sport participation and experience, but different theories have conceptualised social influence in different ways which make it difficult for researchers to examine the relative role of significant others in youth sport. For example, self-determination theory (Deci \& Ryan, 1985, Journal of Research in Personality, 19(2), 109-134) concerns about autonomy support and controlling behaviours, but very little has been researched about how these theory-driven social influences matter to young athletes' sporting experience, in comparison with typical types of social influences such as positive influence (reward, praises, respect, friendliness), punishment, and dysfunction (disrespect, conflicts). This paper aimed to investigate the predictive power of autonomy support and controlling behaviours on young athletes' motivational outcomes, namely competence, effort, enjoyment and trait anxiety, comparing with other typical types of social influences (i.e., positive influence, punishment, and dysfunction). With institutional ethics approval, a total of 440 young athletes (mean age $=14.59, \mathrm{SD}=4.13$, range $=$ $9-18 ; 57.5 \%$ male) in this study completed a package of questionnaires including the Sport Climate Questionnaire (SCQ; Deci, 2001), Controlling Coach Behavior Scale (CCBS; Bartholomew, Ntoumanis, \& Thogersen-Ntoumani, 2010, Journal of Sport and Exercise Psychology, 32(2), 193-216), Perceived Social Influence in Sport Scale-2, and scales that measured the four motivational outcomes. Hierarchical multiple regression showed that autonomy support and positive influence can predict competence, effort and enjoyment in all social agents significantly $\left(R^{2}=.12-.36, P\right.$ $<.01)$. Positive influence is more important in predicting effort among all social agents $\left(\sqrt{ } \Delta \mathrm{R}^{2}=.20-.25\right)$ than autonomy support $\left(\sqrt{ } \Delta R^{2}=.16-.22\right)$. Positive influence from mother is a superior predictor on enjoyment (positive influence: $\sqrt{ } \Delta R^{2}=.23$; autonomy support: $\sqrt{ } \Delta R^{2}=.22$ ). Punishment, dysfunction, and coaches' controlling behaviours can predict effort, enjoyment and trait anxiety significantly $\left(\mathrm{R}^{2}=.03-.10, P<.01\right)$. Dysfunction performed better on predicting effort $\left(\sqrt{ } \Delta R^{2}=-.25\right)$ and enjoyment ( $\sqrt{ } \Delta \mathrm{R}^{2}=-.13$ ) than coaches' controlling behaviours (not significant). In conclusion, social influences conceptualised in self-determination theory have unique predictive power on young athletes' sporting experience, but the prediction is improved when positive influence, punishment, and dysfunction are taken into account. An integrated framework that includes multiple theoretical concepts of social influence is warranted for further investigation in future research.

D1.P44. Motivating adult outpatients with major depressive disorder towards physical activity: a self-determination approach

IOANNIS MORRES ${ }^{1 *}$, ANTONIS HATZIGEORGIADIS ${ }^{1}$, NIKOS COMOUTOS ${ }^{1}$, DIMITRIOS PLOUMPIDIS ${ }^{2}$, MARINA ECONOMOU ${ }^{2,3}$, EIRINI SIDERI ${ }^{2}$, ALEXANDROS MARIDAKIS $^{4}$, MARIA STAMOULI ${ }^{3}$, KATERINA PIKOULI ${ }^{3}$, MANOLIS LOUKADAKIS ${ }^{3}$ \& YANNIS THEODORAKIS ${ }^{1}$

\footnotetext{
${ }^{1}$ University of Thessaly, ${ }^{2}$ National and Kapodistrian University of Athens, ${ }^{3}$ Byron-Kaissariani Community Mental Health Centre, ${ }^{4}$ Eginition Day Hospital *Corresponding author: iomorres@pe.uth.gr @Morresloannis
}

Despite the antidepressant effects of physical activity, depressed patients show a sedentary lifestyle. Previous studies have reported that self-determination theory (SDT) (Ryan \& Deci, 2017, Self-determination theory: Basic Psychological Needs in Motivation Development and 
Wellness. New York: Guilford) is a promising theoretical platform with respect to motivational enhancement towards physical activity in mental health settings. However, these studies reflected partially on the SDT model, did not measure depression, and recruited mental health patients with mixed diagnoses who were mainly physically active. Therefore, the purpose of this study was to explore whether the SDT model comprising controlling and autonomous behavioural regulators and the psychological needs of competence, autonomy and relatedness is capable of predicting physical activity participation in major depressed adult outpatients. After ethical approval by the University of Thessaly, recruitment was conducted at the Byron-Kaissariani Community Mental Health Centre, Athens, Greece. Main inclusion criteria referred to age range of 18-65 and formal psychiatric diagnosis of major depression as a primary disorder. Measurements involved the Beck Depression Inventory (BDI), Stage of Change (SOC), International Physical Activity Questionnaire (IPAQ) and the SDT model referred to Psychological Needs and Behavioural Regulation in exercise. Structural equation modelling examined motivation processes to physical activity participation. A total of 206 moderately adult depressed outpatients participated in the study (mean age: $45.89 \pm 12.37$; mean BDI: $21.32 \pm$ 10.90). The results showed that (a) need satisfaction predicted positively SOC and neutralized the mediating positive effect of autonomous behavioural regulation; (b) need satisfaction predicted positively SOC and the corresponding selfreported metabolic equivalents measured by IPAQ; and (c) when depression was added to the latter model, it showed an adverse effect on need satisfaction and no effect on other constructs. The three models explained $18 \%, 15 \%$, and $18 \%$ of the variance, respectively. Our findings suggest that facilitating satisfaction of the needs of competence, autonomy and relatedness for exercise seems to be the key and preceding step towards motivational enhancement to physical activity participation among depressed patients. Synergetic fostering of patients' psychological needs by health professionals is essential in order to bring about the maximum effect; specifically, creating optimal challenges for physical activity participation while promoting individual initiatives and supporting feelings of social belonging may increase the needs of competence, autonomy, and relatedness, respectively (Morres et al., 2014, In A.G. Papaioannou \& D. Hackfort (Eds.), Physical exercise and major depressive disorder in adult patients (pp. 823-834). London: Taylor \& Francis).

\section{D1.P45. Analysis of individual perceptional differences of potentially relevant trust components in sports teams}

\section{CHRISTINA PLATH*}

\section{University of Vechta}

*Corresponding author: christina.plath@uni-vechta.de

Because of its function as a complexity-reducing mechanism trust plays a very important role for interpersonal as well as collective interactions in all contexts (Luhmann, 2014, Vertrauen. Ein Mechanismus der Reduktion sozialer Komplexität. Konstanz: UVK). According to a differential psychological perspective trust is the result of the complex relationship between personal and situational factors, so it is context-sensitive. While there are theoretical approaches and empirical research to the importance on organizational trust as well as for school contexts there are only a few surveys within the sports area (Dreiskämper, 2015, Die Vertrauenswürdigkeit der Anti-Doping Arbeit von Sportverbänden. Münster: WWU; Mayer et al., 1995, Academy of Management Review, 20, 709-734; Schweer, M., 2017, In Schweer (Ed.), Lehrer-Schüler-Interaktion (pp. 523-545). Wiesbaden: VS). Therefore the positive influences of trust especially for performance enhancement as well as the working atmosphere are discussed. In contrast there is a lack of research that examines the subjective perception of the relevance of different trust components and individual differences in team sports. As well there is an absence of theoretical approach that considers the diversity of the involved team members either. This survey tries to reduce this gap by examine the individual differences on trust components in the context of volleyball sports in a qualitative research design. With institutional ethics approval, 12 qualitative structured interviews ( $f=6, m=6$ ) were conducted and transcribed. The analysis occurred according to the method of empirical based construction of types (Kelle \& Kluge, 2010, Vom Einzelfall zum Typus. Fallvergleich und Fallkontrastierung in der qualitativen Sozialforschung. Wiesbaden: VS). The analysis identifies four comparative dimensions (implied trust theory, effects when trust is available, effects when trust is missing, antecedents of trust). Based on this dimensions the analysis indicates four qualitatively different types of trust (task-focused type, taskand-social-focused type, undifferentiated type, hierarchical-controlling type). The method of empirical based construction of types allows an investigative approach to examine specific aspects of trust. The results of these systematic differences are the foundation of the different identified types. The results illustrate individual differences with regard to the general relevance of trust as well as to the specific aspects for volleyball teams. The findings clarify the importance of context-sensitive trust and its aspects. Referring to this the individual needs and differences of the team members should be considered in training and competition situations.

D1.P46. Is giving up good for you? Goal adjustment capacities as a mediating link between hope theory, sport enjoyment and life satisfaction

JON RADCLIFFE*, PAUL DUTTON \& NINA FRYER

\author{
Leeds Trinity University \\ ${ }^{*}$ Corresponding author: J.Radcliffe@leedstrinity.ac.uk
}

Aligned with positive psychology, Hope Theory (Snyder, 2002, Psychological Inquiry, 13, 249-275) proposes that hopeful individuals achieve their goals because they are able to set goals, derive different pathways to achievement and believe they are the agents of change. Athletes with high levels of hope may therefore be driven to persevere in the pursuit of a target despite such becoming unobtainable or inappropriate and be reluctant to adjust a given objective. Goal adjustment within sport can promote positive wellbeing and failing to give up on 
goals which are unachievable may result in psychological distress (Nicholls et al., 2016, Psychology of Sport and Exercise, 27, 47-55). However, it may not be easy for a sporting participant to 'give up' (Smedema, Chan, \& Phillips, 2014, Rehabilitation Psychology, 59, 399-406), because of 'must win' cultures, where giving up may be seen as weakness (Nicholls et al., 2016, Psychology of Sport and Exercise, 27, 47-55). This study investigated whether hope affected athletes' ability to disengage from unachievable goals, and relationships between 'givingup', life satisfaction and sport enjoyment. Participants were recruited voluntarily from sports clubs across Yorkshire. Following ethical approval, 233 participants (female: $n=171$; male: $n=61$; mean age: $42.5 \pm 9.5$ years) completed an on-line questionnaire, which was analysed using SPSS 21. Four inventories were used: Goal Disengagement and Reengagement Questionnaire (GDRQ), State Hope Scale (SHS), Physical Activity Enjoyment Scale Questionnaire (PACES) and the Satisfaction with Life Scale (SWLS). Analysis showed high hope was positively related to low goal disengagement $(r=$ $-.21, P<.001)$. Furthermore, high goal disengagement correlated negatively with both sport enjoyment $(r=-.21, P<.001)$ and life satisfaction $(r=-.24, P<.001)$. However, high levels of hope predicted higher sport enjoyment levels $(r=.36, P<$ .001). Results suggest that athletes with high hope find goal disengagement challenging, thus helping sports professionals and coaches to understand why some athletes find goal disengagement more difficult than others. Thus, high levels of hope may be beneficial when objectives are achievable, but problematic when goals are unobtainable. Understanding hope theory can help athletes and coaches manage goal disengagement by demonstrating that giving up in sport may not be an admission of defeat, but a facilitator for improved sport enjoyment and life satisfaction.

D1.P47. Career support services in Europe: current status and future challenges

\section{NICKY VAN ROSSEM*, PAUL WYLLEMAN, SIMON DEFRUYT \& KOEN DE BRANDT}

\section{Vrije Universiteit Brussel}

*Corresponding author: nicky.van.rossem@vub.be
In order to optimize their vocational development, employability and employment, active and former elite athletes face different challenges and obstacles (Wylleman \& Rosier, 2016. A holistic perspective on the development of elite athletes. In Raab, Wylleman, Seiler, Elbe, \& Hatzigeorgiadis (Eds.), Sport and exercise psychology research: From theory to practice. Elsevier). Although the EU Guidelines on Dual Careers of Athletes (European Union, 2012, EU guidelines on dual careers of athletes: Recommended policy actions in support of dual careers in high-performance sport) created an overview of actions with the aim of developing sustainable dual career arrangements, recent studies report the high demand for more scientific knowledge related to career support services of (former) elite athletes. The current study aimed at (1) identifying the roles and interactions of career support services in 6 European countries, (2) comparing the roles and cooperation of career support services between the types of organisations and countries involved. The study is part of the 'Be a Winner In Sport and Employment before and after athletic Retirement' (BWISER) project, co-funded by the Erasmus+ Programme of the European Union. With institutional ethics approval, an online survey was organized in 6 different European countries: Belgium, Germany, Italy, Slovenia, Spain, and Sweden. The participants consisted of career support providers that (at least indirectly) provided support to active or former elite athletes to optimize their employability and/or employment before and/or after the athletic career termination. Career support providers from 4 types of organisations (i.e. representative bodies, elite sport organisations, employment agencies and educational institutions) were included within the sample. Results reveal significant differences in the amount and specific types of support between the different types of organisations, showing small up to moderate effect sizes. Moreover, the amount and structure of cooperation between the different organisations concerned with career support appear to be highly dependent on national context. Suggestions for optimisation of cooperation between stakeholders in a (dual) career support environment will be discussed, considering current organisation- and country-specific findings. Based on the findings of this study, avenues for further research focussing on improving the career support services in Europe will be shared. 


\section{Day 1. Posters - Sport and Performance}

\author{
D1.P48. Categorising symptoms and types of yips \\ in athletes
}

SALLY AKEHURST*, PHILIP CLARKE \& DAVID SHEFFIELD

\author{
University of Derby \\ *Corresponding author: s.akehurst@derby.ac.uk
}

Yips are a well known phenomenon in golf and may relate to similar phenomenon in other sports such as target panic in archery, but no corroborating evidence exists. Previously three types of yips, Type I (physiological based), Type II (psychological based) and Type III (both physiological and psychological based; Clarke et al., 2015, International Review of Sport and Exercise Psychology, 8, 156-184) have been proposed along with characteristic symptoms that have been experienced. However, there is no comprehensive list of symptoms for each yips category supported through first-hand athlete experience, thus providing the aim of this study. Fifty-eight athletes (30 golfers and 28 archers), aged over 18 years, completed an online survey consisting choking and yips demographic self-report forms, which required participants to identify themselves as having experienced choking and/or yips, or not, and select symptoms they had experienced from a pre-informed checklist, adding any others not listed. Two physiological and four psychological symptoms were the most commonly experienced ranging 26-37 out of 58 reports by affected athletes. Multiple Chi Square tests of independence were performed revealing significant associations for: (A) sport and yips-type, revealing that $0 \%$ of golfers were categorised as Type I compared to $27.6 \%$ of archers, $10 \%$ of golfers as Type II similar to $10.3 \%$ of archers, and $90 \%$ of golfers as Type III in comparison to $62.1 \%$ of archers; (B) sport and symptoms, revealing golfers as more likely to experience eight of the symptoms (Four physiological and four psychological), and (C) yips type and symptoms, 11 in particular (Five physiological and Six psychological), revealing those categorised as Type III, as more likely to experience all symptoms. No additional symptoms were added to the 19 pre-informed list, thus, it is considered a comprehensive checklist of symptoms for the yips, offering practitioners, coaches and athletes guidance with regard potential warning signs. Jerks and loss of control of limbs were the most prevalent physiological symptoms for Type I and III yips, whereas increased negativity, self-consciousness, nervous and anxiety were the most prevalent psychological symptoms for Type II and III yips. Golfers were more likely to experience both physiological and psychological symptoms compared to archers for whom Type I yips are more prevalent than Type II. Findings support target panic as a form of yips and the categorization of Type III yips, which was revealed as most prevalent across both sports.

\section{D1.P49. Performance analysis of trail running in undulating terrain}

GLENN BJÖRKLUND ${ }^{1,2^{*}}$, DENNIS BORN ${ }^{3}$, THOMAS STÖGGL ${ }^{4} \&$ MIKAEL SWARÉN ${ }^{5}$

\author{
${ }^{1}$ Swedish Sports Confederation, ${ }^{2}$ Mid Sweden University, \\ ${ }^{3}$ Würzburg University, ${ }^{4}$ Salzburg University, ${ }^{5}$ Royal \\ Institute of Technology \\ *Corresponding author: glenn.bjorklund@rf.se
}

Compared to track running, trail running is more technical challenging as the running consists of sections with various inclinations and surfaces. In addition, the terrain (Townshend, Worringham, \& Stewart, 2010, Medicine \& Science in Sports \& Exercise, 42, 160-169) and surface (Jensen, Johansen, \& Kärkkäinen, Sports Science, 1999, 17, 945-950) influence the runner's performance. The aim was to identify and analyse the specific sections where runners lose and gain time, when running in undulating terrain and to investigate laboratory variables and their relation to trail running performance. With institutional ethics approval, the participants (17 men and 4 women: mean age: $29 \pm 5$ years; stature: $179.5 \pm$ $0.08 \mathrm{~m}$; body mass: $69.1 \pm 7.4 \mathrm{~kg}$; fat percent: $14.2 \pm 4.8$ ), performed laboratory tests to determine $\mathrm{VO}_{2 \text { max }}$, running economy, and anthropometric characteristics. Two days later, each participant performed an outdoor trail running test, consisting of two laps on a $3.5 \mathrm{~km}$ course with a total elevation gain of $486 \mathrm{~m}$, where kinematics, kinetics and time data were collected. The overall running performance was associated to laboratory data as to absolute and relative $\mathrm{VO}_{2 \max }(r=-0.71$ and -0.82 , both $P$ $<0.01)$ as well as lean body mass $(r=-0.76, P<0.01)$ and fat percent $(r=0.84, P<0.001)$. There was a decrease in performance of $88 \pm 37 \mathrm{~s}$ from the first to the second lap $(d=0.71)$. The time lost at the different sections was dependent on the terrain $(F=19.1, P<$ $\left.0.001, \eta^{2}=0.614\right)$, showing that the runners lost most of their times on the three major uphill sections (Uphill 1 $=29.0 \pm 18.5 \mathrm{~s}$, Uphill $2=18.9 \pm 7.5 \mathrm{~s}$, Uphill $3=19.7 \pm$ $\left.14.2 \mathrm{~s}, P>0.05, \eta^{2}=0.136\right)$. The largest variation in performance was in the downhill sections with a coefficient of variation between 14-32\%. During the most technical downhill run the stride frequency increased when the ascent was steeper $\left(F=107.2, P<0.01, \eta^{2}=\right.$ $0.931)$. These results show that the intra-individual performance reduction mainly occurs in the uphill sections where the overall performance is related to $\mathrm{VO}_{2 \max }$, lean body mass and fat percent. However, the largest variation between individuals is during downhill running. Further, the present study suggests that during steeper downhills an increased stride frequency is a key factor to maintain speed, which has not been shown previously.

\section{D1.P50. Do challenge and threat states predict attentional control and performance during a pressurised soccer penalty task?}

JACK BRIMMELL ${ }^{1}$, JOHN PARKER ${ }^{1}$, MARK WILSON $^{2}$, SAMUEL VINE ${ }^{2} \&$ LEE MOORE ${ }^{3}$ 
${ }^{1}$ University of Gloucestershire, ${ }^{2}$ University of Exeter, ${ }^{3}$ University of Bath

*Corresponding author: s1204709@connect.glos.ac.uk

Under pressure, a challenge state leads to better sports performance than a threat state (Moore et al., 2013, Journal of Sport Exercise Psychology, 35, 551-562). The integrative framework of stress, attention, and visuomotor performance attributes this to a challenge state resulting in optimal goaldirected attention, and a threat state resulting in sub-optimal stimulus-driven attention and greater distractibility by threatening stimuli (Vine et al., 2016, Frontiers in Psychology, 7, 1671). However, to date, little research has tested these predictions. Thus, this study examined whether challenge and threat states influenced performance and attentional control during a pressurised soccer penalty task. With institutional ethical approval, 42 participants ( 35 male, mean age: $23.50 \pm$ 6.62 years) completed the single-trial pressurised soccer penalty task. Before the task, challenge and threat states were measured via cardiovascular reactivity to task instructions. During the task, a mobile eye tracker was used to record attentional control. After the task, performance (distance from the centre of the goal $[\mathrm{cm}]$ ) and attentional control (quiet eye duration [ms] or final fixation before the initiation of the run-up, and percentage viewing time on the goalkeeper) were determined via video analysis. The results revealed that cardiovascular reactivity predicted task performance $\left(R^{2}=.278, \beta=.546, P<.001,95 \% \mathrm{Cl}=10.14\right.$ to 32.05$)$, with a cardiovascular response more representative of a challenge state associated with greater accuracy. Furthermore, cardiovascular reactivity marginally predicted quiet eye duration $\left(R^{2}=.071, \beta=.314, P=.066,95 \% \mathrm{Cl}=-1.35\right.$ to 40.16$)$, with a cardiovascular response more indicative of a challenge state linked to longer quiet eye durations. Finally, cardiovascular reactivity predicted the percentage of time spent fixating on the goalkeeper $\left(R^{2}=.091, \beta=.343, P=.044,95 \% \mathrm{Cl}=\right.$ .02 to 1.58 ), with a cardiovascular response more reflective of a challenge state associated with more time fixating on the goalkeeper. The results support previous research showing that a challenge state benefits sports performance under pressure, and suggest that a challenge state might aid performance by encouraging longer quiet eye durations or optimising goal-directed attentional control (Moore et al., 2012, Psychophysiology, 49, 1417-1425). However, contrary to predictions, a threat state did not result in greater attention to the goalkeeper or threatening stimuli. Therefore, more research is required to test the predictions of the integrative framework of stress, attention, and visuomotor performance.

\section{D1.P51. Quantitative skill provision in undergraduate sport and exercise science programmes}

\section{SCOTT BURNET*}

\section{Southampton Solent University \\ *Corresponding author: scott.burnet@solent.ac.uk @Scott_Burnet}

Performance sport has seen considerable advances due to growth and development in science, medicine and technology, with an unprecedented ability to collect and process performance-related data (Drawer \& Brown, 2014, The Sport and Exercise Scientist, 40, 28-30). It is therefore vital that future Sport and Exercise Scientists have proficient quantitative skills (QS) which has been defined as numeracy, data handling, mathematics and statistics and their application in a given context (Wilson, 2013, International Journal of Mathematical Education in Science and Technology, 44, 796807). Whilst undergraduate Sport and Exercise Science (SES) programmes have seen increased capacity to collect quantitative data, many students struggle to meet to the QS demands of their programme of study. The aim of the study was to investigate the QS provision in UK SES programmes. With institutional ethical approval, a survey based research design was conducted, with the University and Colleges Admissions Service (UCAS) database used to identify single-honours SES programmes in the UK. Entry requirements relating to mathematics and science were obtained from all 88 higher education provider (HEP's) offering a SES programme of study. Programme specifications and module descriptors relating to mathematics, statistics or research methods based modules were retrieved from $43 \%$ of the HEP's offering a SES programme. Sixty-two percent of SES programmes required or preferred a science or mathematics A-level or equivalent as an entry requirement, although $43 \%$ stated that physical education and psychology were accepted as a science. In contrast, $38 \%$ of UK SES programmes required points and a mathematics $C$ grade at GCSE for entry onto their programme of study. Only $23 \%$ of programmes offered some form of quantitative-specific or scientifically-orientated module throughout the 3-4 y undergraduate programme. The remaining programmes delivered QS provision within generic research methods modules, with $77 \%$ typically delivering a module during each year of study. One-third of modules surveyed were equivalent to less than 20 credits. Whilst there is a clear need for SES graduates to be proficient and confident in handling and interpreting quantitative data, the exposure that SES students have to quantitatively-orientated modules is varied and inconsistent both in terms of content and the delivery pattern of the content. Whilst future research will look to examine the non-curriculum-based support that students receive throughout their degree programme, it was felt that greater QS attention was required to enhance the proficiency with which SES students handle numerical data.

\section{D1.P52. Acute effects of kinesiology tape application on skin blood flow: considerations in the management of athletic performance}

\section{SARAH CATLOW*, TANDIWE WALLACE-SIMS \& LANCE DOGGART}

\section{Plymouth Marjon University \\ *Corresponding author: scatlow@marjon.ac.uk}

The use of Kinesiology tape (K-tape) is evident across all levels of sport and exercise participation. Manufacturers claim that $\mathrm{K}$ tape application can increase blood and lymphatic flow, decrease pain from injury and increase proprioception (Korman et al., 2015, Biomedical Research International, 1, 1-5). There is a dearth of empirical evidence to support these claims. The aim of the study was to assess 
the acute effects of kinesiology tape application on skin blood flow around the gastrocnemius muscle. Following University ethics approval 20 injury free participants (mean age: $21.30 \pm 2.49$ years) attended the laboratory on two occasions, $24 \mathrm{~h}$ apart, incorporating four conditions. The conditions included: no tape (NT); instant tape application (ITA); tape application plus $24 \mathrm{~h}(\mathrm{TA}+24 \mathrm{~h})$; and instant tape removal post $24 \mathrm{~h}(\mathrm{ITR}+24 \mathrm{~h})$. For the tape condition a $20 \mathrm{~cm}$ length of tape was applied originating $2.5 \mathrm{~cm}$ below the popliteal fossa and stretched down the midline of the gastrocnemius with a $50 \%$ stretch as per the recommendations of Doggart and Catlow (2015, Journal of Sports Sciences, 33, 110-111). Blood flow was measured using a Laser Doppler flow monitor (Moor Instruments VMS-LDF, UK) with the disc positioned on the inferior aspect of the muscle avoiding interference with the tape. Blood flow was recorded in blood perfusion units (BPUs) averaged over a $10 \mathrm{~min}$ period for each condition (Bosiers \& Schneider, 2009, Critical Limb ischemia. New York: Informa Healthcare). A repeated measures ANOVA, with post-hoc Bonferroni, was used to analyse the data across the four conditions. The mean ( $\pm s$ ) BPU for each condition was noted as 42.7 BPU ( $s=21.4), 34.9 \mathrm{BPU}$ ( $\mathrm{s}$ = 17.6), 34.9 BPU ( $s=16.8), 32.7 \mathrm{BPU}(\mathrm{s}=16.9)$ for NT, ITA, TA $+24 \mathrm{~h}$ and ITR+24 $\mathrm{h}$ respectively. The repeated measures ANOVA revealed significant differences in BPUs across the four conditions $(P<0.05$; ES $=0.2$ ). Bonferroni post-hoc analysis noted significant differences between the NT and ITA $(P<0.05 ; \mathrm{ES}=0.4 ; 95 \% \mathrm{Cl}=2.2-13.1 \mathrm{BPU})$ and the NT and ITR+24 h $(P<0.05 ; \mathrm{ES}=0.4 ; 95 \% \mathrm{Cl}=0.7-18.6 \mathrm{BPU})$ conditions only. The results of this study suggest that the immediate application of $\mathrm{K}$ tape significantly decreased skin blood flow, measured in BPUs, around the gastrocnemius and continued to maintain a reduced skin blood flow post $24 \mathrm{~h}$ and following the removal of the tape after $24 \mathrm{~h}$. It would therefore appear that the application of $\mathrm{K}$ tape to a non-injured lower leg muscular site prohibits skin blood flow around the site, across a $24 \mathrm{~h}$ period, and may impact negatively on athletic performance during this time.

\section{D1.P53. A Christmas break temporarily improves the perception of well-being in academy soccer players}

\section{NEIL CLARKE ${ }^{1 *}$, MATTHEW ELLIS ${ }^{2}$, PETER MUNDY ${ }^{1} \&$ MARK NOON $^{1}$}

\section{${ }^{1}$ Coventry University, ${ }^{2}$ Coventry City FC \\ *Corresponding author: neil.clarke@coventry.ac.uk @Clarke_ND}

During the English elite youth soccer season, a training break is generally observed at Christmas. The aim of the present study was to assess the effect of an eight day Christmas break on academy soccer player's perceptions of well-being. Eighteen academy soccer players (mean \pm SD: age: $17 \pm 1$ years; stature: $180 \pm 6 \mathrm{~cm}$; body mass: $71.2 \pm 6.7 \mathrm{~kg}$ ) completed a perception of well-being questionnaire every day during a 28-day period incorporating ten days before (pre) and after (post) an eight day Christmas break. All players and parents provided written informed consent following institutional ethical approval. At 09:00 prior to training, each player was asked to rate their perceptions of seven items, each on a seven-point scale [very good $(+3)$, normal $(0)$ to very poor $(-3)]$, to monitor their perceptions of well-being related to: motivation to train, quality of previous night's sleep, quality of recovery from previous day, appetite, feeling of fatigue, level of stress and level of lower-body muscle soreness (Noon et al., 2015, Journal of Sports Sciences, 3, 2106-2115). All players were given instructions on the use of the questionnaire by the sport science staff and familiarised with the process of completing the questionnaire throughout the previous six months. The questionnaire data were typically not normally distributed so an Analysis of Variance (ANOVA) with a bootstrapping procedure of 1000 replications was used to assess any differences between the training blocks. Furthermore, confidence intervals and effect sizes using partial eta squared $\left(\eta_{\mathrm{p}}^{2}\right)$ and Cohen's $d$ were calculated. During the Christmas break small to large improvements in perceptions of wellbeing were evident [sleep quality (vs. pre: $P=0.002 ; 95 \% \mathrm{Cl}$ : $0.14,0.57 ; d=1.25$; vs. post: $P=0.004 ; 95 \% \mathrm{Cl}: 0.12,0.57 ; d=$ 0.60 ), recovery (vs. pre: $P=0.069 ; 95 \% \mathrm{Cl}:-0.01,0.41 ; d=0.75$; vs. post: $P=0.009 ; 95 \% \mathrm{Cl}: 0.10,0.51 ; d=0.98$ ), fatigue (vs. pre: $P=0.002 ; 95 \% \mathrm{Cl}: 0.15,0.63 ; d=1.09 ;$ vs. post: $P=0.001 ; 95 \% \mathrm{Cl}$ : $0.30,0.81 ; d=1.27$ ), stress (vs. pre: $P=0.014 ; 95 \% \mathrm{Cl}: 0.06,0.37$; $d=1.05$; vs. post: $P=0.008 ; 95 \% \mathrm{Cl}: 0.07,0.41 ; d=1.04)$ and lower-body muscle soreness (vs. pre: $P=0.001 ; 95 \% \mathrm{Cl}$ : 0.36 , $0.81 ; d=1.42 ;$ vs. post: $P=0.001 ; 95 \% \mathrm{Cl}: 0.66,1.12 ; d=1.42)$ ]. However, only trivial to small differences were observed between pre- and post-Christmas break $(P>0.05$; $d=0.02$ $0.37)$. Only trivial differences in motivation $\left(P=0.912 ; \eta_{\mathrm{P}}^{2}=\right.$ $0.00)$ and appetite $\left(P=0.671 ; \eta_{\mathrm{P}}^{2}=00\right)$ were observed between any training block. In conclusion, a mid-season Christmas break temporarily improves perceptions of well-being in academy soccer players, but this is not maintained once training recommences.

\section{D1.P54. The physiological demands of riding for horse riders}

\section{DANIELLE FLOOD ${ }^{1 *}$, RACHEL WHITE ${ }^{1}$, STEVE ATKINS ${ }^{2} \&$ SARAH HOBBS $^{3}$}

\section{${ }^{1}$ Myerscough College, ${ }^{2}$ University of Salford, ${ }^{3}$ University of Central Lancashire \\ *Corresponding author: dflood@uclan.ac.uk/ \\ dflood2729@student.myerscough.ac.uk}

The repetitive motions used by the rider to control the horse, dampen the vertical and horizontal acceleration of the horse's trunk, and yet maintaining good horse riding posture cannot be easily replicated on simulation or exercise equipment. As such, determining the physiological demands of horse riding requires a mixed approach of laboratory and field testing. Previous studies have quantified oxygen uptake for different gaits (Westerling, 1983, European Journal of Applied Physiology and Occupational Physiology, 50(3), 373-382) and heart rates during race riding (Trowbridge et al., 1995, European Journal of Applied Physiology and Occupational Physiology, 70(1), 66-69) when riding one horse, but different horses may necessitate different physiological demands. The aims of the this study were to establish baseline fitness data of student riders, and compare the difference in heart rate of the riders when riding two different horses at three common riding gaits; walk, trot and canter. With institutional ethical approval, participants ( $n=$ 
19), performed a standardised exercise test in the form of a 6 min Astrand-Rhyming Cycle Ergometer Test to assess fitness. Two ridden 45 min sessions one week apart, which incorporated each of the three gaits were carried out on two different horses for each rider to assess the physiological demands of different horses. Mean heart rate for each gait, each rider and each session were analysed using ANOVA. The mean heart rates for each gait were; walk 131.89 bpm, trot 132.49 bpm, and canter $145.65 \mathrm{bpm}$. Gait had a significant effect on the heart rate of the riders $(P=0.001)$. There was no significant effect of the rider $(P=0.256)$ or the horse $(P=0.374)$. A post hoc pairwise Fisher comparison showed a significant difference $(P<$ $0.05)$ between walk and canter and trot and canter. There was no significant difference $(P>0.05)$ between walk and trot. The results suggest that gait is the most significant factor when measuring heart rate, highlighting that gait indicates the intensity of the exercise, unlike the horse's way of going, which did not show any significant effect on the heart rate of the rider. Therefore, to improve the cardiovascular fitness of riders, the canter gait should be encouraged.

D1.P55. Effect of uphill and level high intensity exercises on energy cost of running, blood lactate and respiratory exchange ratio

FERNANDO GONZÁLEZ-MOHÍNO*, DANIEL JUÁREZ, INMACULADA YUSTRES AMORES \& JOSÉ MARÍA GONZÁLEZ RAVÉ

\section{University of Castilla-La Mancha \\ *Corresponding author: fernando.gmayoralas@uclm.es @Fer742}

Warm up is an accepted practice preceding nearly every athletic event such as running. However, there is little research about high intensity warm up effectiveness. In order to recommend a high intensity exercise during warm-up protocol, we should know its effects on the physiology of the runner. The aim of this study was to assess effects of high intensity exercise (uphill or level gradient) on energy cost of running $(\mathrm{Cr})$, blood lactate and respiratory exchange ratio (RER). With institutional ethics approval, eleven well-trained distance runners (mean $\pm \mathrm{SD}$ : $\mathrm{VO}_{2}$ max $68.05 \pm 4.78 \mathrm{ml} \cdot \mathrm{kg}^{-1} \cdot \mathrm{min}^{-1}$, age $25.18 \pm 3.9$ years, weight $65.36 \pm 6.8 \mathrm{~kg}$, height $177.0 \pm 0.05 \mathrm{~cm})$ performed an uphill high intensity exercise $(6 \times 6$-s uphill strides at $105 \%$ $\mathrm{vVO}_{2}$ max and $5 \%$ gradient) and level high intensity exercise ( $9 \times 20$-s level strides at $105 \% \mathrm{vVO}_{2}$ max and $1 \%$ gradient) in a randomized cross-over design. Before and $10 \mathrm{~min}$ after (passive recovery) of both high intensity exercise, the participants involved a 5 -min submaximal run at $60 \% \mathrm{vVO}_{2}$ max (1\% gradient) for measure $\mathrm{Cr}$ and RER and blood lactate. Exercise resulted in a $\mathrm{Cr}$ significantly increase $(P<0.05)$ after of level and uphill high intensity exercise in $1.43 \%$ and $4.27 \%$, respectively. Blood lactate significantly increased ( $P$ $<0.001)$ in both high intensity exercise $(1.04 \pm 0.40$ vs 3.35 $\pm 1.06 \mathrm{mmol} \mathrm{I}^{-1}$ and $0.86 \pm 0.29$ vs $2.32 \pm 0.76 \mathrm{mmol} \mathrm{I}^{-1}$, level and uphill, respectively). RER significantly decreased ( $P$ $<0.05$ ) by $9.9 \%$ and $5.6 \%$ in level and uphill exercise. The results suggest that both high intensity exercise deteriorate running economy. Recently, it has been found a decrease of running economy due to an increase of blood lactate
(Hoff, Storen, Finstad, Wang, \& Helgerud, 2016, Journal of Strength \& Conditioning Research, 30, 1373-1378) similar to our study. Another possible explanation is the change of substrate utilization as indicated by the decreased of RER (Collins et al., 2000, Journal of Sports Sciences, 18, 83-90; Zavorsky, Montgomery, \& Pearsall, 1998, European Journal of Applied Physiology and Occupational Physiology, 77, 224-230), would increase dependency on fat oxidation and as result, increase oxygen demand (Burke et al., 2017, The Journal of Physiology, 595, 2785-2807). On the base of this data, for prescribing a high intensity warm-up protocol, it is necessary a long transient phase (more than 10 min like our study) to return to basal levels and in order to avoid impairing the subsequent performance.

\section{D1.P56. Relation of race discipline to overall performance in draft legal, and draft illegal world amateur sprint triathlon}

\section{MARK HORNE*}

\section{Richmond American International University} *Corresponding author: mark.horne@richmond.ac.uk @markjameshorne

Findings from draft-legal Olympic distance triathlon typically show that the run discipline accounts for the highest proportion of variance in overall performance time when compared to other triathlon disciplines. The run accounts for between $71 \%$ and $91 \%$ of total variance in finish times (Fröhlich et al., 2014, Journal of Athletic Enhancement, 5:2; Fröhlich et al., 2013, International Journal of Kinesiology \& Sports Science, 15:1(3)). Drafting is an energy efficient method of performing a sporting activity where an athlete performs in a position which shelters them from drag. Drafting whilst cycling has been shown to reduce energy expenditure when compared to cycling in an unsheltered position (Hausswirth et al., 2001, Medicine \& Science in Sports Exercise. March 1, 33(3), 485-492). Until a rule change in early 2016, International Triathlon Union (ITU) sanctioned amateur triathlon did not allow drafting in the cycle discipline. The purpose of this analysis was twofold. Firstly to determine predictors of overall performance in draft-legal sprint triathlon, and secondly to compare draft-legal sprint race data, with published draft-illegal sprint race data (Horne, 2017, International Journal of Sports Science \& Coaching, in press). Stepwise regressions with the triathlon race disciplines (Swim, Cycle, Run) as predictor variables, and Overall Time as an outcome variable were conducted using data for 25-29 year-old athletes at the draft-legal ITU 2016 Sprint distance Age-group World Championships, and compared to data from the draft-illegal 2015 Age-group Sprint World Championships. This study utilised publicly available data from the results section of the ITU website (which was then anonymised). Permission to use the data was sought and granted from the ITU. In the 2015 draftillegal races, the cycle discipline explained the greatest proportion of variance in overall finish time for both males and females (Male $R^{2}=.59, P<.001$ : Female $R^{2}=$ $.69, P<.001)$. However, in the 2016 draft-legal races, running accounted for the greatest proportion of variance in both male and female races (Male $R^{2}=.63, P<.001$; 
Female $\left.R^{2}=.78, P<.001\right)$. Therefore, in draft-legal racing sprint distance triathlon shows a similar pattern of results to draft-legal Olympic distance racing. This differs from draftillegal racing, where cycling, rather than running predicts a greater proportion of overall variance. Therefore coaches of international age-group athletes should seek to tailor training and race plans to the drafting status of target races.

\section{D1.P57. Is there an optimal load for the} development of muscular power in the straight bar deadlift?

\section{CHELSEY LAWSON ${ }^{1 *}$, MARK LYONS ${ }^{2} \&$ MICHAEL J. DUNCAN $^{1}$}

${ }^{1}$ Coventry University, ${ }^{2}$ University of Limerick

*Corresponding author: ac2444@coventry.ac.uk

The development of muscular power is an important aspect within training to improve athletic performance (Mcbride et al., 2002, The Journal of Strength and Conditioning Research, 16, 75-82). The concept of optimal loading to develop peak power output is regularly discussed within the literature (Soriano et al., 2015, Sports Medicine, 45, 1191-1205). However there is conflict as to which "load" might optimise peak power with studies citing loads from $<30 \%$ (Cormie et al., 2007, Medicine and Science in Sports and Exercise, 39, 340-349) to $>70 \%$ of 1RM (Comfort et al., 2012, Journal of Strength and Conditioning Research, 26, 2970-2974). To date, no studies have examined peak power using the deadlift. The aim of this study was therefore to identify if an optimal load for peak power output (PP) for the straight bar deadlift (DL).13 male strength trained adults (mean age \pm S.D $=$ $25.1 \pm 5.6$ ) participated in this study, following ethics approval and informed consent. Each participant attended the performance laboratory on two occasions to (1) determine their DL $1 \mathrm{RM}$ and (2) perform DL repetitions at loads of 20-90\% 1RM 3 repetitions per load, each separated by 3 mins). PP, was assessed using a TENDO FitroDyne V-207 (Tendo Sports Machines, Slovak Republic), averaged for each of the 3 repetitions per load. Repeated measures ANOVA revealed a significant difference in PP output across loads $20-90 \%(P=0.005)$. Post-hoc analyses indicated power output at 20 and 30\% 1RM was significantly lower compared to loads $40-70 \% 1 \mathrm{RM}$ (all $P<0.05$ ). PP increased at $40 \% 1 \mathrm{RM}$ and was similar across the intensities of $40-70 \%$ (all $P>0.05$ ). PP at $90 \% 1 \mathrm{RM}$ was significantly lower than at $40-70 \% 1 \mathrm{RM}(P<0.05)$ but not different to $20-30 \% 1 \mathrm{RM}(P>0.05)$. The results suggest that there is no single intensity were PP is optimised during the straight bar $\mathrm{DL}$, contrary to prior studies (Cormie et al., 2007; Comfort et al., 2012) which examined different exercises. The results of this study do indicate that PP may be maximised during $\mathrm{DL}$ in the range 40-70\%1RM.

D1.P58. Injury rate differences among genders in U.S. community rugby-7s

VICTOR LOPEZ JR ${ }^{1,2^{*}}$, RICHARD MA ${ }^{1,2,3}$, MERYLE G. WEINSTEIN ${ }^{1,4}$, PATRIA A. HUME ${ }^{2}$, ROBERT C. CANTU ${ }^{5,6}$,

\section{CHIRSTIAN VICTORIA ${ }^{1,7}$, TARA CONDON ${ }^{1,8}$ \& ANSWORTH A. ALLEN ${ }^{9,10}$}

${ }^{1}$ Rugby Research and Injury Prevention Group, Hospital for Special Surgery, ${ }^{2}$ Auckland University of Technology, Sports Performance Research Institute New Zealand,

${ }^{3}$ University of Missouri, Missouri Orthopaedic Institute \& Comparative Orthopaedic Laboratory, ${ }^{4}$ New York University, Steinhardt School of Culture, Education and Human Development, ${ }^{5}$ Emerson Hospital, ${ }^{6}$ World Rugby, Independent Concussion Group, ${ }^{7}$ New York University, Global Institute of Public Health, ${ }^{8}$ University of North Carolina, ${ }^{9}$ Hospital for Special Surgery, ${ }^{10}$ National Basketball Association, New York Knickerbockers *Corresponding author: drvictorlopezjr@gmail.com @rripg

Rugby-7s has seen a recent surge in popularity, globally, resulting in its 2016 Olympics debut. There is a lack of Rugby-7s injury rate data, with a significant head/neck injury rate reported in amateur to elite U.S. Rugby-7s cohort (Lopez et al., 2012, American Journal of Sports Medicine, 40, 179-184; Lopez et al., 2016, Medicine \& Science in Sports \& Exercise, 48, 1320-1330; Ma et al., 2016, Medicine \& Science in Sports \& Exercise, 48, 1957-1966). Only the above studies by this group, have utilized the international consensus statement on rugby injuries, to evaluate injury rates in U.S. National Rugby-7s events considered a strategic rugby market (Fuller et al., 2007, British Journal of Sports Medicine, 41, 328-331). The aim of this study was to characterize gender injury rates in U.S. Rugby-7s, amateur (U-19) to elite national candidates. With institutional ethics approval, prospective time-loss injuries in USA Rugby tournaments (2010-2013) were collected among 13,644 U.S. players (Men =9,768; Women $=$ 3,876; age: 13-49 years) were included over 28 tournaments ( 37 days) of 2,688 matches (Men $=1,886$; Women $=802$ ) in 1,137 sides/teams (Men = 814; Women $=323$ ). Injury incidence (per 1000 player-hour (ph)) and mechanism were captured using the Rugby Injury Survey \& Evaluation (RISE) methodology (e.g. Lopez et al., 2012). Time-loss injuries were defined injuries where the players did not return to play the day of their injury. Injury severity was defined as days absent before return to training/competition (including post tournament). Overall incidence of time-loss injuries was 50.6/1000 ph. Time-loss injuries were highest among sub-elite level players (69.5/1000ph) $(P<$ 0.001 ). Fifty percent of all injuries occurred in 20-25 year olds which was consistent with the age distribution of the participants. Average injury severity was 44 days ( $\mathrm{Cl} 37.7-$ 49.4) for the $97 \%$ of players with follow-up data. Most tournament match injuries were new acute injuries (95\%), occurred during tackling (68\%), were ligament sprains $(35 \%)$, and in the lower extremity (52\%). Head/face $(22.6 \%)$ and upper extremity $(24.7 \%)$ injuries were also frequent. The U.S. amateur population had a lower injury incidence, as compared to international elite cohorts, however, they had a higher frequency of upper extremity and head/face injuries (Fuller et al., 2010, Clinical Journal of Sports Medicine, 20, 179-184). U.S. Rugby-7s head/neck injuries $(22 \%)$ were greater than international elite male Rugby-7s (5\%) (e.g. Fuller et al., 2010). U.S. injury prevention should focus on head/neck injuries in the tackle. 
Evaluating U.S. tackling techniques, may guide future training protocols to reduce injury.

\section{D1.P59. The validity and reliability of bilateral torque measured using Garmin Vector pedals.}

\section{KELLY MURRAY* \& FLORENTINA HETTINGA}

\author{
University of Essex \\ ${ }^{*}$ Corresponding author: kamurray@essex.ac.uk \\ @kmurraysportsci
}

The Garmin Vector pedals (GVP's) are considered to be a valid and reliable device for the measurement of power output during cycling across a range of intensities, when compared to an SRM (Novak \& Dascombe, 2016, Measurement in Physical Activity and Exercise Science, 20, 167-172) (Nimmerichter, Schnitzer, Prinz, Simon, \& Wirth, 2017, International Journal of Sports Medicine, 38, 439-446). The GVP's consist of strain gauges which measure torque at both the left (LP) and right (RP) pedals. Measuring bilateral torque allows coaches and scientists to evaluate cyclists for bilateral asymmetries, which could influence performance (Bini and Hume, 2014, Journal of Sports Physiology and Performance, 9, 876-881). However, to be able to determine whether a cyclist is producing torque asymmetrically, left and right GVP's should be assessed, independently, for their accuracy and repeatability. Therefore, the aim of this study was to assess the validity and reliability of torque measurements from both left and right GVP's. To eliminate the influence of human variability, this aim was investigated using static load testing. A unicycle, with the seat post fixed horizontally to a surface, was used as a cycling rig. GVP's were attached to the cranks, and each pedal was rotated and fixed at crank angles of $0^{\circ}$ to $360^{\circ}$, with steps of $45^{\circ}$. At each crank angle, each pedal was loaded with $0,8,16$, 24,32 , and $40 \mathrm{~kg}$, using hook weights. Known crank loads and angles were used to calculate expected (TRUE) torque values, which were compared against those measured by both left and right GVP's using 95\% limits of agreement (LoA). The LoA between left and right pedal torque measurements were also assessed. The 6 loads were applied to each pedal 3 times, at all crank angles, to calculate the reliability as the coefficient of variation (COV). Bland Altman analysis shows a mean bias of torque for LP vs. TRUE of $0.11 \mathrm{~N} . \mathrm{m}(95 \% \mathrm{Cl}:-0.75$ and 0.97 N.m), for RP vs. TRUE of -0.04 N.m (95\% Cl: -1.25 and $1.18 \mathrm{~N} . \mathrm{m})$ and LP vs. RP of $0.15 \mathrm{~N} . \mathrm{m}$ (95\% Cl: -0.95 and 1.24 N.m). COV was $<1.5 \%$ for $L P$, and $<2 \%$ for RP. These results demonstrate a good agreement between TRUE torque and torque measured by both LP and RP, as well as a good agreement between LP and RP. These findings suggest that GVP's are valid and reliable in the assessment of bilateral torque, and therefore could be used to assess bilateral asymmetries during cycling.

D1.P60. Is there a correlation between GPS and Tracab outputs for physical performance markers within soccer?

\section{JOSHUA RICE*}

\section{Liverpool John Moores Univerisity \\ *Corresponding author: j.rice@2014.ljmu.ac.uk}

Background: Demands of modern day soccer have increased, increasing the challenge for players; to both recover and then reproduce typical match outputs in forthcoming fixtures. Subsequently player tracking is required to; better understand the demands of practice maximise performance and minimize injury risk and optimise training load patterns. An accurate way of measuring the above is required throughout the game week, with the integration of MEMS (StatSports Viper) and TracAb against each other key. Objectives: To understand whether there is a correlation between MEMS and TracAb outputs for physical performance markers within soccer. Methods: 9 Professional footballers (mean \pm SD: age $27 \pm 4$, body mass $78.2 \pm 8.7 \mathrm{~kg}$, height $\pm 181.9 \pm 7.3 \mathrm{~cm}$ ) participated in this study. Data was collected simultaneously from both MEMS; StatSports Viper $(10 \mathrm{~Hz}) \operatorname{TracAb}(25 \mathrm{~Hz})$ and downloaded for analysis after the completion of the game. Metrics analysed for correlation; High intensity distance, High speed running distance, sprint distance, total distance covered, high metabolic load distance, explosive distance, decelerations, accelerations and total number of sprints. Results: (1) All workload measures that were quantified had no significant difference allowing for integration of the two systems against each other; (2) the correlations between the TracAb system and the MEMS technology all showed strong positive correlation (>0.94). Conclusions: Findings allow for practitioners to interchangeably use physical performance outputs from both MEMS (wearable technology) with the semi automated camera tracking (TracAb) system to create a periodized training week for the players. 


\section{Day 2. Free Communications - Biomechanics and Motor Behaviour}

\author{
D2.S4.1(1). Footstrike kinematic effects of motor \\ learning strategies to retrain running gait
}

\section{MARIANNE GITTOES ${ }^{1 *}$, KELLY ASHFORD ${ }^{1}$, CHARLOTTE MICHELMORE $^{2} \&$ ISABEL MOORE ${ }^{1}$ \\ ${ }^{1}$ Cardiff Metropolitan University, ${ }^{2}$ Cardiff University \\ *Corresponding author: magittoes@cardiffmet.ac.uk}

Running gait retraining, which uses motor learning strategies, is increasingly being advocated as an effective rehabilitation approach to allow runners to mitigate the risks of re-injury and to remain physically active (Davis \& Crowell, 2011, Clinical Biomechanics, 26, 78-83). By providing gait retraining cues, lower-limb running kinematics at footstrike may be altered to affect the mechanical risks of overuse running injuries. The aim of this study was to develop insight into the influence of a short-term gait retraining strategy on the footstrike kinematics of rearfoot-striking runners. With institutional ethical approval, lower-limb kinematics were tracked using CODAmotion V6.79.3 (Charnwood Dynamics Ltd, Leicestershire, UK) analysis during treadmill running conditions for six recreational, habitually rearfoot-striking runners (mean \pm SD age: $24.8 \pm 6.2$ years; height $1.69 \pm$ $0.08 \mathrm{~m}$; body mass $67.9 \pm 7.1 \mathrm{~kg}$ ). Each participant completed three, six minute running conditions (10 kilometres per hour) separated by a five minute rest period. A normal $(\mathrm{N})$ condition and two intervention conditions using the verbal cues of "run like you are trying to squash oranges" ( $O$ ) and "run quietly" (Q) were completed in a randomised order. Sagittal and frontal plane kinematics were derived during the last minute of each six minute running condition. Footstrike was defined using the vertical acceleration data of the first contacting foot marker. While step frequency was maintained (mean \pm SD all footstrikes: $1.40 \pm 0.11 \mathrm{~Hz}, \mathrm{n}=159$ ) across conditions, the $\mathrm{O}$ condition elicited a shallower foot orientation (difference: $\mathrm{N}=7.6^{\circ}$, effect size $[\mathrm{ES}]=0.66$; $\mathrm{Q}=6.0^{\circ}, \mathrm{ES}=0.51$ ) compared to the other conditions. While cueing had a small effect on the foot inversioneversion angle (difference: $\mathrm{O}$ to $\mathrm{N}=1.3^{\circ}, \mathrm{ES}=0.23$; $\mathrm{O}$ to $\left.\mathrm{Q}=1.0^{\circ}, \mathrm{ES}=0.18\right)$, moderate effects on the rearfoot orientation were evidenced (difference: $\mathrm{O}$ to $\mathrm{N}=3.2^{\circ}$, ES $=0.61 ; \mathrm{O}$ to $\mathrm{Q}=2.9^{\circ}, \mathrm{ES}=0.50$ ). More proximally, the two cueing conditions had large effects on the knee extension at footstrike compared to the $\mathrm{N}$ condition (O to N ES $=0.82 ; \mathrm{Q}$ to $\mathrm{N} \mathrm{ES}=0.82$ ). Conditional responses indicated the potential to elicit mechanical transitions in habitually rearfoot-striking runners using an analogy learning strategy. Short-term gait retraining strategies may accordingly be implemented in rehabilitation programmes to alleviate the kinematic risk of overuse running injury. Consideration of the associated physiological effects of the respective retraining programme are however, advocated.
D2.S4.1(2). Influence of range of motion on muscle activity whilst using the MuJo ${ }^{\mathrm{TM}}$ External Shoulder Machine

\section{VICTORIA JONES*, PETER MUNDY, NEIL CLARKE \& SAMUEL OXFORD}

\section{Coventry University \\ *Corresponding author: jonesv10@coventry.ac.uk @VJones160}

Resistance equipment is often restricted to a single plane of movement. However, multi-planar movements may be more effective, by facilitating the development of motor strategy and intermuscular coordination. Due to its moving axis cam technology, the MuJo ${ }^{\mathrm{TM}}$ External Shoulder Machine enables such movement, with independent loading of multiple joints and muscles. Furthermore, the range of motion (ROM) over which the upper limb travels can be adjusted to target specific muscles, which may have useful implications within a rehabilitation environment. However, little is currently known about how altering the ROM affects the activity of the shoulder musculature whilst using the device. Therefore, the purpose of this study was to determine the effect of altering the ROM on muscle activity during exercises performed on the MuJo ${ }^{\mathrm{TM}}$ External Shoulder Machine. Based upon an a priori power analysis ( $f=0.40, a=$ $0.05, \beta=0.80$ ), and following institutional ethics approval, nine recreationally-active male participants (means \pm SDs: age: $25 \pm 5$ years; body mass:77.06 $\pm 11.06 \mathrm{~kg}$; height:1.76 $\pm 0.09 \mathrm{~m}$ ) performed abduction and external rotation of the shoulder at twelve different ranges of motion, in a randomised, counterbalanced order. Surface electromyography (EMG) was collected from the upper trapezius, anterior and posterior deltoids, infraspinatus, and latissimus dorsi. Muscle activity was normalised to the peak activity from a 1 repetition maximum test, also performed on the machine. The effect of abduction and rotation angle on normalised peak EMG was analysed via a two-way analysis of variance with repeated measures for each muscle; effect sizes were calculated using partial eta squared $\left(\eta^{2}{ }_{p}\right)$. A significant main effect for abduction in the upper trapezius was found $\left(F_{(1.1,8.7)}=15.34, P=0.003\right)$, with a moderate effect size $\left(\eta_{p}^{2}=0.66\right)$. Bonferroni-adjusted post-hoc analysis demonstrated that all angles of abduction were significantly different from one another (95\% Cl: $90^{\circ} / 60^{\circ}$ [3.97, 26.97]; 60\% $/ 30^{\circ}$ [1.37, 22.84]); $\left.30^{\circ} / 90^{\circ}[-48.41,-6.77]\right)$. A significant main effect for rotation in the latissimus dorsi was found $\left(F_{(3,24)}=7.96, P=\right.$ $0.001)$, with a moderate effect size $\left(\eta_{p}^{2}=0.50\right)$; post-hoc tests revealed $0^{\circ}$ to be significantly lower than both $90^{\circ}(95 \% \mathrm{Cl}[0.04$, $26.80])$ and $60^{\circ}(95 \% \mathrm{Cl}[0.79,16.98])$. No significant interactions or main effects were found in the remaining muscles. In conclusion, altering the ROM results in some changes in muscle activity during abduction and rotation, perhaps indicating a greater requirement for stabilisation during the less constrained repetitions. Further studies incorporating kinematics may provide deeper understanding into the effects on motor strategy that may occur when exercising with this device. 
D2.S4.1(3). The effect of knee joint angle on quadriceps explosive strength and underpinning neural and contractile determinants

\section{MARCEL B. LANZA*, THOMAS BALSHAW \& JONATHAN FOLLAND}

\section{Loughborough University \\ *Corresponding author: m.bahia-lanza@lboro.ac.uk}

Knee extension strength (maximum voluntary torque, MVT) is known to exhibit a pronounced angle-torque relationship (de Ruiter et al., 2007, Journal of Applied Physiology, 97, 1964-1701). However, little is known about the effect of joint angle on explosive strength, the ability to rapidly increase torque from rest, or the underpinning neural and contractile determinants. This study aimed to compare quadriceps isometric explosive torque, neural drive and muscle intrinsic contractile properties at 5 different knee joint angles $\left(35^{\circ}, 50^{\circ}, 65^{\circ}, 80^{\circ}\right.$ and $95^{\circ} ; 0^{\circ}$ was full knee extension). Following institutional ethics approval and familiarisation, thirty-four young healthy males ( $22 \pm 3$ years; $1.77 \pm 0.08 \mathrm{~m} ; 74 \pm 7 \mathrm{~kg}$ ) performed two experimental sessions with a counterbalanced order. Each session involved 2 maximum, and 6-8 explosive, voluntary contractions at each angle; with recordings of torque (MVT and explosive torque at 50, 100 and $150 \mathrm{~ms}$ after contraction onset) and quadriceps surface EMG. Supramaximal femoral nerve stimulation was used to evoke octet contractions ( 8 pulses at $300 \mathrm{~Hz}$ ). Due to the pronounced influence of joint angle on maximum torque we focused on the ability to express the available torque explosively at each angle (i.e. explosive torque relative to MVT). Whilst there were some isolated subtle differences in relative explosive torque with knee joint angle $(<9 \%)$, there was no systematic increase or decrease with joint angle. Relative explosive torque at $150 \mathrm{~ms}$ at $65^{\circ}$ was lower than the other angles and at $100 \mathrm{~ms}$ $95^{\circ}$ was higher than $80^{\circ}$ and $65^{\circ}$ and $80^{\circ}$ also was higher than $65^{\circ}$ ( $P \leq 0.04$ ). Normalised EMG (to Mmax) for 0-100 ms and 0-150 $\mathrm{ms}$ was effected by joint angle being higher at more flexed angles $(P \leq 0.011)$. In contrast, octet $T_{50}$ was higher at the most extended positions $\left(35^{\circ}\right.$ and $50^{\circ} ; \mathrm{P} \leq 0.01$ ). In conclusion, relative explosive voluntary torque did not show a systematic change across joint angles, which may be a consequence of opposite effects of joint angle on neural drive (higher at more flexed positions) and the intrinsic contractile properties (evoked octet, higher at more extended positions).

\section{D2.S4.1(4). Neural and hypertrophic adaptations to maximal eccentric versus concentric training matched for total work}

\section{SUMIAKI MAEO ${ }^{1,2^{*}}$, XIYAO SHAN $^{1}$, SHUN OTSUKA ${ }^{1}$ HIROAKI KANEHISA ${ }^{3}$ \& YASUO KAWAKAMI ${ }^{1}$ \\ ${ }^{1}$ Waseda University, ${ }^{2}$ Loughborough University, \\ ${ }^{3}$ National Institute of Fitness and Sports \\ *Corresponding author: smaeo1985@gmail.com}

Eccentric training is known to induce greater muscle hypertrophy than concentric training. However, it is poorly understood whether the superiority of eccentric training is due to higher torques that can be produced during eccentric contractions, or due to resulting greater total work in a training program. Furthermore, time-course of neural and hypertrophic adaptations and their contributions to strength gains following eccentric versus concentric training are unclear. Therefore, this study aimed to elucidate neural and hypertrophic adaptations following maximal eccentric versus concentric training matched for total work. With institutional ethics approval, twelve young males conducted single-joint isokinetic $(180 \% \mathrm{~s})$ maximal eccentric contractions of the knee extensors in one leg (ECC-leg) and concentric in the other (CON-leg), 6 sets/session (3-5 sets in the initial 1-3 sessions), 2 sessions/week for 10 weeks. The preceding leg, which was switched every session, performed 10 repetitions/ set, and the following leg conducted an equivalent volume of work. In addition to maximal torque during training, agonist electromyogram (EMG) and magnetic resonance imaging (MRI)-based anatomical cross-sectional area (ACSA) and transverse relaxation time (T2) as reflective of neural drive, hypertrophy and edema, respectively, were assessed weekly throughout the training period and at pre and post. Total work did not differ between legs $(\sim 185 \mathrm{~kJ})$, but total repetitions were significantly $(P<0.05)$ less for ECC-leg $(985$ \pm 38 reps) than CON-leg (1358 \pm 70 reps). Torque and EMG (in the trained contraction condition) significantly increased in both legs after week 1 and 4, respectively, with a greater degree for ECC-leg (torque $72 \pm 56 \%$ and EMG $73 \pm 58 \%$ at post) than CON-leg (34 $\pm 30 \%$ and $20 \pm 24 \%)$. ACSA significantly increased after week 4 in ECC-leg only (3.8\% at post), without significant $\mathrm{T} 2$ changes throughout. Percent changes $(\% \Delta)$ in torque through training were highly correlated to $\%$ $\triangle E M G$ for both ECC-leg $(r=0.756-0.906$ at all weeks and post) and CON-leg ( $r=0.606-0.776$ at weeks $1,2,4,8$ and post), but not to \% $\triangle \mathrm{ACSA}$ (except for one time point in CONleg: $r=0.776$ at week 4). Multiple regression analysis revealed that $\% \triangle E M G$ solely explained $53-80 \%$ and $30-56 \%$ of the total variance in \% $\Delta$ torque through training in ECC-leg and CON-leg, respectively. These results suggest that eccentric training produces greater neural and hypertrophic changes than concentric training even when matched for total work, while most of the strength gains during 10-wk training is attributable to the increased neural drive.

D2.S4.1(5). Influence of a visual reference point for the hurdle on step regulation and hurdle kinematics in gymnastics handspring vault

\section{APOSTOLOS S. THEODOROU* \& GEORGIOS NTALLAS}

\section{National and Kapodistrian University of Athens \\ *Corresponding author: aptheod@phed.uoa.gr}

Hurdle step is the transitional phase between the gymnasts' run-up and the springboard, with the constraint of adjusting sprinting body position to one from which they can initiate takeoff without losses in velocity (Hay, J.G., 1978, The biomechanics of sports techniques (second edition) (pp. 279-329). Englewood Cliffs NJ: Prentice-Hall). The purpose of the study was to investigate if a visible takeoff point for the hurdle at the end of the approach run would influence the onset of visual regulation and hurdle kinematics. Following institutional ethical approval, 10 elite male gymnasts (age $23.4 \pm 4.9$ years, height $1.68 \pm 0.06 \mathrm{~m}$, mass 63.3 $\pm 6.2 \mathrm{~kg}$ ) performed, in a random order, 6 handspring vaults with a full approach run under two conditions interspersed 
with a $48 \mathrm{~h}$ rest: (a) with a $5 \mathrm{~cm}$ white tape on the runway (distance customized for each athlete) marking the last touchdown of the approach run and commencement of the hurdle (tape condition - T), and (b) under the usual regulations of the event (non-tape condition - NT). The runway was marked and spatiotemporal data from the 10th up to the last step of the approach and the hurdle were collected by 4 stationary cameras (sampling rate 300 $\mathrm{fps}$ ). The maximum toe-spring-board distance variability $\left(T S B V_{\max }\right)$, the onset of step regulation (Berg \& Mark, 2005, Human Movement Science, 24, 496-531), the centre of mass horizontal velocity $\left(V X_{t o}\right)$, vertical velocity $\left(V y_{t o}\right)$, takeoff velocity $\left(V_{\text {to }}\right)$ and angle of takeoff $\left(\theta_{\text {to }}\right)$ at the instant of takeoff for the hurdle were measured. In both conditions step regulation commenced on the 5th last support (4th last step) of the approach at a TSBV $\max$ of $18.48 \pm 8.85 \mathrm{~cm}$ and $18.80 \pm 12.56 \mathrm{~cm}$ for NT and T condition respectively. At $T$ condition, $V X_{\text {to }}\left(9.06 \pm 0.41{\left.\mathrm{~m} . \mathrm{sec}^{-1}\right)}^{-1}\right.$ and $V_{\text {to }}(9.35 \pm$ $0.37 \mathrm{~m} . \mathrm{sec}^{-1}$ ) increased significantly compared to NT condition $\left(V X_{\text {to }}=8.85 \pm 0.49 \mathrm{~m} \cdot \mathrm{sec}^{-1}, P=0.007, d=1.10 ; V_{\text {to }}=\right.$ $\left.9.11 \pm 0.47 \mathrm{~m} . \mathrm{sec}^{-1}, P=0.009, d=1.05\right)$. Although the absolute values of $V y_{\text {to }}$.and $\theta_{\text {to }}$ increased in $T$ condition, no significant differences were found between conditions $\left(V y_{\mathrm{to}}: \mathrm{T}=2.27 \pm 0.42 \mathrm{~m} \cdot \mathrm{sec}^{-1}\right.$ vs NT $=2.13 \pm 0.18 \mathrm{~m} \cdot \mathrm{sec}^{-1}$; $\theta_{\mathrm{to}}: \mathrm{T}=14.11^{\mathrm{O}} \pm 2.84$ vs NT $=13.57 \pm 1.48^{\mathrm{O}}$ ). The results suggest that introducing a visible takeoff point for the hurdle does not alter the onset of step regulation but significantly improves the kinematics of the hurdle. 


\section{Day 2. Free Communications - Physiology and Nutrition}

D2.S4.2(1). Effects of consuming whey and casein protein post resistance exercise on food intake and fullness in healthy males

\section{DAVID BROOM ${ }^{1 *}$, ALEX KERRIGAN $^{1}$, ELLY BOT ${ }^{1}$, LOUIS HAVENHAND ${ }^{1} \&$ LEWIS JAMES ${ }^{2}$ \\ ${ }^{1}$ Sheffield Hallam University, ${ }^{2}$ Loughborough University *Corresponding author: D.R.Broom@shu.ac.uk}

Whey and casein protein are commonly consumed after resistance exercise (Benelam, 2009, Nutrition Bulletin, 34, 126-173). At rest, whey protein consumption has been shown to decrease subsequent food intake compared to casein protein (Hall et al., 2003, British Journal of Nutrition, 89, 239-248), but little is known about the appetitive effects of whey and casein after resistance exercise. We hypothesized that whey protein would have a greater satiating effect than casein when consumed after resistance exercise. Following institutional ethical approval, 21 healthy, physically active males volunteered for this double blind, randomized, repeated measures, crossover study. After an overnight fast, having controlled their diet and refraining from vigorous activity the day before, participants consumed a standardised breakfast consisting of cereal bar and semiskimmed milk ( $15 \%$ of predicted Total Energy Expenditure) at home at $8 \mathrm{am}$. On arrival at the laboratory, fullness was measured using Visual Analogue Scales (VAS, 0-100 mm) at baseline $(10 \mathrm{am})$ and frequently throughout the trials. The resistance training protocol consisted of 6 exercises targeting all major muscle groups for 3 sets of 10 reps at $85 \%$ of 10-RM, calculated during familiarisation. Post resistance exercise $(11 \mathrm{am})$, after a standardised shaking protocol, participants consumed whey or casein protein (matched for $20 \mathrm{~g}$ protein) added to $400 \mathrm{~mL}$ of water. At $12 \mathrm{pm}$, participants were escorted to feeding booths in close proximity and provided with an ad-libitum pasta lunch. Whole-wheat fusilli pasta and Waitrose tomato and basil pasta sauce were measured using weighing scales and prepared using standardized procedures to control temperature, texture and water absorption. Participants were monitored until $12.30 \mathrm{pm}$ and after leaving the laboratory, fullness VAS were completed at $1 \mathrm{pm}$ and $2 \mathrm{pm}$. After checking normality using the ShapiroWilks test, food intake was analysed using a paired samples t-test and fullness analysed using repeated measures ANOVA with statistical significance accepted if $P<0.05$. There was no significant difference in food intake between the whey and casein trial (Whey $802 \pm 192 \mathrm{~g}$ vs Casein $856 \pm 201 \mathrm{~g} ; P>$ $0.05 ; \mathrm{dz}=0.34)$. There were no trial effects $(F(1,20)=1.107$; $P>0.05)$ or interaction for fullness $(F(5,100)=1.217 ; P>0.05)$ but there was an effect of time $(F(3,57)=41.054 ; P<0.001)$. These results suggest that when consumed immediately after resistance exercise whey and casein exert similar effects on subsequent appetite and food intake.
D2.S4.2(2). Cycling using immersive virtual reality enhances acute exercise tolerance

\author{
JAMIE HIGHTON ${ }^{1 *}$, THOMAS WILLIAMS ${ }^{1,2}$, GERARD \\ NOWLAN ${ }^{1,3}$, SERBAN POP $^{1} \&$ CRAIG TWIST $^{1}$ \\ ${ }^{1}$ University of Chester, ${ }^{2}$ University of Portsmouth, ${ }^{3}$ Sport \\ Ireland \\ *Corresponding author: j.highton@chester.ac.uk \\ @JHighton
}

Incorporating virtual reality into exercise, using a third person view of an avatar on a screen, can improve affective responses and performance (Murray et al., 2016, Psychology of Sport and Exercise, 22, 328-336). However, the physiological responses to exercise with virtual reality are unclear, whilst the effects of immersive virtual reality technology during exercise have not been explored. The aim of this study was to examine the physiological and perceptual responses to an endurance capacity test with the use of immersive virtual reality. After ethical approval, fifteen recreationally active participants (mean age: $24.9 \pm$ 4.2 years; stature: $176.7 \pm 3.4 \mathrm{~cm}$; body mass: $77.7 \pm 6.1$ $\mathrm{kg}$; $\mathrm{VO}_{2 \text { max }}: 53.1 \pm 6.3 \mathrm{ml} \cdot \mathrm{kg}^{-1} \cdot \mathrm{min}^{-1}$ ) completed two experimental trials of a cycle ergometer time-to-exhaustion test at $80 \% \mathrm{VO}_{2 \max }$ in a randomised crossover design. In one trial (VR), participants completed a laboratorybased cycle ergometer test whilst wearing a stereoscopic virtual reality headset and headphones. The virtual reality environment in this condition was designed to mimic cycling on an open, flat road. In another trial, participants completed the same cycle ergometer test but wore no headset or headphones (CON). Cardio-respiratory data and perceived exertion were measured throughout each trial, whilst affect and enjoyment were assessed after completing exercise using the International Positive and Negative Affect Schedule Short Form and Physical Activity Enjoyment Scale, respectively. All variables were analysed using magnitude-based inferences (ES $\pm 90 \%$ confidence intervals). There was a very likely moderate increase in time to exhaustion ( $E S=0.65 \pm 0.29)$ in VR $(561 \pm 158$ s) compared to CON (468 $\pm 135 \mathrm{~s})$. There were no clear or meaningful differences in oxygen uptake ( $E S=0.11 \pm$ $0.23)$, minute ventilation $(0.03 \pm 016)$, heart rate $(0.12 \pm$ $0.33)$ or blood lactate $(0.03 \pm 0.48)$ between VR and CON at 6 min of exercise (a time that all participants achieved before exhaustion). However, RPE was likely lower (ES = $0.52 \pm 0.48)$ in VR $(17.9 \pm 1.2)$ than CON $(18.6 \pm 0.8)$ at 6 $\min$. Furthermore, post-exercise positive affect $(\mathrm{ES}=0.52$ \pm 0.4 ) and exercise enjoyment ( $E S=0.82 \pm 0.47$ ) was likely higher in VR. Virtual reality can improve exercise tolerance, which is likely due to a reduced perception of exertion and an improved affective response to exercise rather than physiological changes. 
D2.S4.2(3). Reliability of ratings of perceived exertion during combined arm-leg ergometry

\section{MATHEW HILL ${ }^{1 *}$, MICHAEL PUDDIFORD ${ }^{2}$, CHRISTOPHER $\mathrm{TALBOT}^{2}$ \& MIKE PRICE ${ }^{1}$ \\ ${ }^{1}$ Coventry University, ${ }^{2}$ University of Northampton \\ *Corresponding author: matt.hill@coventry.ac.uk @DrMathewHill}

Rating of perceived exertion (RPE; Borg, 1982, Medicine and Science in Sports and Exercise, 14, 377-381) is a reliable method of assessing exercise intensity during arm-cranking (ACE) and cycling (CYC) (Eston \& Williams, 1988, British Journal of Sports Medicine, 22, 153-155). Although previous studies have investigated the cardiorespiratory responses to combined arm-leg ergometry (ALE), the reliability of RPE during this novel exercise is unknown. Therefore, the aim of this study was to assess the reliability of RPE for the prescription of exercise intensity during ALE. Following institutional ethical approval, twelve males (24.6 \pm 5.3 , years) initially undertook incremental exercise tests to volitional exhaustion for ACE $(133 \pm 14 \mathrm{~W})$ and CYC $(253 \pm 32$ W). On three subsequent occasions, participants undertook ALE using two modified Monark ergometers involving three bouts of exercise at RPE 9, 13 and 17, in that order. Each participant was allowed $4 \mathrm{~min}$ to adjust arm and leg power output to match the assigned RPE value. Power output was then clamped for the fifth minute of each RPE level. Participants were blind to power output and cadence throughout trials. Heart rate (HR), oxygen uptake $\left(\mathrm{V}_{2}\right)$, pulmonary ventilation $\left(\dot{\mathrm{V}}_{\mathrm{E}}\right)$ and overall ratings of perceived exertion $\left(\mathrm{RPE}_{\mathrm{O}}\right)$ were recorded in the 5 th min after power output was clamped. The validity of the RPE scale as a means of producing different exercise intensities was assessed using two-way (RPE $\times$ trial) analysis of variance. Intertrial variability was assessed using intraclass correlation coefficients (ICC). No significant differences were observed for $\mathrm{HR}(P=0.874), \mathrm{VO}_{2}$ $(P=0.772), \dot{\mathrm{V}}_{\mathrm{E}}(P=0.631)$ and $\mathrm{RPE}_{\mathrm{O}}(P=0.723)$ between trials (all $d=0.1-0.2)$, whereas significant differences were observed between each level of RPE (all $P<0.001, d>1.0$ ). When asked to produce exercise intensities equating to RPE 9,13 and 17 , the ALE trials elicited $52 \pm 9 \%, 70 \pm 9 \%$ and $93 \pm 10 \% \mathrm{VO}_{2 \text { PEAK }}$ of CYC trials, respectively. The ICC for HR was $.938, .910$ and .914 for RPE 9, 13 and 17, respectively. The ICC for $\mathrm{VO}_{2}$ was $.573, .521$ and .922 for RPE 9,13 and 17, respectively. RPE can be used as a reliable frame of reference for the production of exercise intensity during ALE without any formal familiarisation. Since ALE elicits a greater energy expenditure than arm or leg work alone, this novel mode of non-weight bearing exercise might prove effective for aerobic conditioning and weight control.

\section{D2.S4.2(4). Physiological responses to motor learning strategies to retrain running gait: a pilot study}

\section{ISABEL MOORE*, KELLY ASHFORD, CHARLOTTE BITCHELL \& MARIANNE GITTOES}

\section{Cardiff Metropolitan University \\ *Corresponding author: imoore@cardiffmet.ac.uk @izzymoorePhD}

Running gait retraining has been proposed as a rehabilitation strategy for running-related overuse injuries (Barton et al.,
2016, British Journal of Sports Medicine, 50, 513-526) and uses motor learning strategies to facilitate changes in running gait. Whilst risk-reducing effects in biomechanical indicators of overuse injury have been evidenced, the concurrent effects on physiological responses are unclear. The aim of the study was to develop an insight into the physiological responses to running gait retraining strategies commonly used in injury rehabilitation. With institutional ethics approval, nine recreational, rearfoot striking runners (mean \pm SD age: $24 \pm 5$ years; height $1.70 \pm 7.4 \mathrm{~m}$; body mass $68.3 \pm 6.2 \mathrm{~kg}$ ) completed three conditions of treadmill running $(6 \mathrm{~min})$ at $10 \mathrm{~km} \cdot \mathrm{hr}^{-1}$, with a fivemin rest period between conditions. A normal running condition and two intervention conditions using the verbal cues of "run quietly" (external focus of attention, EFOA) and "run like you are trying to squash oranges" (analogy motor learning, AML) were completed in a randomised order. The verbal cues were presented every $30 \mathrm{~s}$. Mean oxygen consumption and heart rate during the final two min and mean central (cRPE) and peripheral (pRPE) ratings of perceived exertion were obtained during each condition. The AML condition produced the greatest increase in oxygen consumption (2.8\%; effect size [ES] 0.51) and heart rate (3.3\%; ES 0.53) compared to the normal condition ( 34.2 vs. $33.2 \mathrm{ml}^{\mathrm{kg}} \mathrm{kg}^{-1} \mathrm{~min}^{-1}$ ). The EFOA condition had similar oxygen consumption $\left(33.3 \mathrm{ml} . \mathrm{kg}^{-1} \mathrm{~min}^{-1} ;-0.1 \%\right.$; ES $0.04)$ and heart rate $(0.7 \%$; ES 0.11$)$ to the normal condition. Oxygen consumption and heart rate were greater in the AML condition than the EFOA (2.9\%; $0.81 \mathrm{ES}$ and 2.7\%; $0.91 \mathrm{ES}$, respectively). The AML and EFOA conditions had elevated pRPE (5\%) compared to normal condition (11.4, 11.4 and 10.9 respectively), as well as cRPE $(12.4,12.0$ and 11.6 respectively; 6.9 and $3.4 \%)$. An analogy learning strategy was found to have larger effects on physiological indicators of running performance during gait retraining than an external focus of attention, possibly due to underlying biomechanical alterations evidenced in the analogy learning strategy (Gittoes et al., 2017 British Association of Sport and Exercise Science Conference). The respective effects were reflected in perceived central effort levels and partially reflected in perceived peripheral muscular effort levels. Clinicians implementing running gait retraining to reduce biomechanical risk indicators are encouraged to use analogy cues, but to consider reducing running workload due to the physiological responses associated with this motor learning strategy.

\section{D2.S4.2(5). Inter-individual variability in the appetite, acylated ghrelin and total peptide YY response to acute exercise in young men: a replicated cross-over study}

FERNANDA REISTENBACH GOLTZ ${ }^{1}$, ALICE THACKRAY ${ }^{1}$, JAMES KING ${ }^{1}$, JAMES DORLING ${ }^{1}$, GREG ATKINSON ${ }^{2}$ \& DAVID J. STENSEL ${ }^{1 *}$

\section{${ }^{1}$ Loughborough University, ${ }^{2}$ Teesside University ${ }^{*}$ Corresponding author: D.J.Stensel@lboro.ac.uk}

Acute exercise transiently suppresses appetite, which coincides with alterations in appetite-regulatory hormones. Individual variability in these responses is suspected, but replicated trials are needed to quantify them robustly. We examined the reproducibility of appetite and appetite-regulatory hormone responses to acute exercise bouts and quantified the individual differences in responses. With 
institutional ethics approval, we recruited 15 healthy, recreationally-active men; mean (SD) age: 22.9 (3.3) years, body fat: 13.1 (5.9)\%, peak oxygen uptake $\left(\mathrm{V}_{2}\right)$ : 54.9 (6.5) $\mathrm{mL} \cdot \mathrm{kg} \cdot \mathrm{min}^{-1}$. Participants were randomised to different sequences of four trials (two control and two exercise). In both exercise trials, participants completed 60-min of fasted treadmill running at $70 \%$ peak $\dot{\mathrm{V}}_{2}$. Participants rested for the equivalent time in the control trials. Appetite perceptions and circulating concentrations of acylated ghrelin and total peptide YY (PYY) were measured immediately before and after exercise/control interventions. Inter-individual differences were explored by correlating the exercise and control pre $(0 \mathrm{~h})$ to post (1 h) change scores on the two occasions. A within-participant linear mixed model was used to quantify any participant $\times$ response interaction (Senn et al., 2011, Statistical Methods in Medical Research, 20, 657-666). Exercise suppressed appetite perceptions (all ES $\geq 0.88, P<0.001$ ) and acylated ghrelin concentrations (ES $=0.62, P<0.001$ ), whilst elevating total PYY concentrations (ES $=1.49, P<$ 0.001 ) compared with control. For all variables, within-trial
SDs were substantially larger for exercise than control. Moderate-to-large positive correlations were observed between the two sets of control-adjusted exercise responses for acylated ghrelin ( $r=0.57, P=0.025)$, hunger $(r=0.82, P<0.001)$, satisfaction $(r=0.74, P=0.002)$, fullness ( $r=0.55, P=0.035)$ and prospective food consumption (PFC) ( $r=0.54, P=0.036)$. The correlation for total PYY was $0.27(P=0.339)$, but improved to 0.71 ( $P=$ 0.003 ) when a substantial outlier measurement was removed. After adjusting for baseline measurements, participant $\times$ response interactions were found for acylated ghrelin $(P<0.001)$, total PYY $(P=0.012)$, hunger $(P<$ $0.001)$, satisfaction $(P<0.001)$, fullness $(P<0.001)$ and PFC ( $P=0.053)$. Our replicated cross-over study allowed, for the first time, the interaction between participant and acute exercise response in appetite parameters to be quantified. Even after adjustment for individual baseline measurements, recreationally-active men showed individual differences in appetite, acylated ghrelin and total PYY responses to acute exercise bouts beyond any random within-subject variability over time. 


\section{Day 2. Free Communications - Psychology (Session 1)}

\section{D2.S4.3(1). Pupillometry during golf putting: a new window on the cognitive mechanisms underlying Quiet Eye}

\section{MARK CAMPBELL ${ }^{1 *}$, AIDAN MORAN $^{2}$, SEAN SURMON ${ }^{3}$, LIZ BRESSAN ${ }^{3}$ \& IAN KENNY ${ }^{1}$ \\ ${ }^{1}$ University of Limerick, ${ }^{2}$ University College Dublin, \\ ${ }^{3}$ University of Stellenbosch \\ *Corresponding author: mark.campbell@ul.ie @Dr_MarkCampbell}

It has long been established that pupillary responses provide a valid and reliable window on the 'intensity' of mental activity or cognitive effort (Hess \& Polt, 1964; Kahneman \& Beatty, 1966). As these responses are routinely and non-invasively measured during eye-tracking, they constitute a promising tool for the study of the cognitive mechanisms underlying skilled performance. Specifically, larger pupil diameter during equiluminance reflects increased attentional resource allocation. In eye-tracking research, the "quiet eye" (QE) has been consistently shown to be a key predictor of perceptual-motor expertise (Mann, Williams, Ward, \& Janelle, 2007; Vickers, 2009). Unfortunately, despite an abundance of $\mathrm{QE}$ research, there has been a dearth of theorising on the specific purpose of QE or the mechanisms that underlie this distinctive pattern of gaze behaviour with some recent exceptions (Gonzalez, Causer, Miall, Grey, Humphreys, \& Williams, 2017; Klostermann, Kredel, \& Hossber, 2013; Vine, Lee, Wlaters-Symons, \& Wilson, 2015). Therefore, the current study aims to tackle this gap in the literature by measuring the allocation of attentional resources during golf putting using pupilommetry. Participants consisted of 24 golfers ( 11 low skill/13 high skill; mean handicap $17 \& 6.9 ;$ SD 3.61/3.73) undertaking 2 blocks of ten putts - short/easy putts (from $1.83 \mathrm{~m}$ ) and longer more difficult putts (from $3.66 \mathrm{~m}$ ). Functional Data Analysis (FDA) was the optimal statistical technique employed to examine the temporal series of pupil diameter changes during putting. Results indicate that peak pupil dilation directly corresponds to the onset of $\mathrm{QE}$. This finding illustrates that $\mathrm{QE}$ onset is the most cognitively intense time for skilled golfers of different abilities. Finally, as predicted the magnitude of pupillary responses was greater for lesser skilled golfers than higher skilled golfers suggesting that the putting execution task was less cognitively challenging for better golfers. We propose that measuring pupil diameter change during tasks is a fruitful avenue for future research to follow to further advance our understanding of the perception-action variable Quiet Eye.

D2.S4.3(2). Coach, athlete, and relational influences on athletes' attitudes, intentions, and willingness to dope

PAUL FREEMAN ${ }^{1 *}$, DI FU ${ }^{1}$, DANIEL MADIGAN ${ }^{2}$ \& TIM REES ${ }^{3}$

\footnotetext{
${ }^{1}$ University of Essex, ${ }^{2}$ York St John University,

${ }^{3}$ Bournemouth University

*Corresponding author: pfreeman@essex.ac.uk
}

There has been increasing interest in the determinants of athletes' attitudes towards doping, with coaches identified as playing a key role. To address calls for research to better account for the interplay between individual and social factors, two studies were conducted with institutional ethics approval with the aim to use a generalisability theory framework to explore how athletes' thoughts about doping are influenced by their perceptions of coaches. Study 1 examined the magnitude of athlete, coach, and relational components in athletes' attitudes, intentions, and willingness to dope using a fully-crossed design. Study 2 examined the relationships between coaches' attitudes and athletes' attitudes, intentions and willingness to dope, and if a shared identity moderates these relationships at athlete and social levels using a nested design. In Study 1, 135 athletes (regionalinternational level; mean age: $20.1 \pm 2.1$ years) read descriptions of four coaches who had different attitudes towards doping (pro-doping, laissez-faire, doping is ineffective, strong anti-doping). After each script, participants rated their attitudes, intentions, and willingness to dope if they were to work with the coach. Athletes' attitudes, intentions, and willingness all comprised significant athlete (29-38\%) and relational (43-51\%) components. In Study 2, 99 high-level athletes (mean age: $19.9 \pm 1.7$ years) considered three coaches who they had worked with, and completed measures of social identity, their perceptions of the coaches' attitudes, and their own attitudes, intentions and willingness to dope if they were to work solely with each coach. Athletes' attitudes, intentions, and willingness all comprised significant athlete (63-74\%) and social (18-26\%) components. Athletes' perceptions of their coaches' attitudes to doping were consistently related to the athletes' own thoughts at athlete $(r s=.51-.86$, $\left.P_{\mathrm{S}}<.01\right)$ and social $\left(r s=.40-.48, P_{\mathrm{S}}<.01\right)$ levels. Further, social identity moderated the relationship between coach and athlete attitudes at the social level $\left(\Delta R^{2}=.02, P=.01\right)$, with the relationship stronger when athletes strongly identified with particular coaches. These findings demonstrate that athletes' thoughts about doping vary in response to different coaches, these thoughts are associated with athletes' perceptions of the coaches' thoughts and whether they identify with the coaches, and that generalisability theory can provide novel insights for anti-doping research. As athletes respond differently to the same message, a one-size-fits-all approach to anti-doping messages will not be optimal for all athletes, therefore, education programmes should reflect these subtleties and equip coaches with the skills to effectively transmit strong anti-doping attitudes to all athletes.

D2.S4.3(3). Attention, heart rate and skilled motor performance: effects of task difficulty

\section{JENNIFER HENDERSON \& CHRISTOPHER RING}

University of Birmingham

*Corresponding author: jxh569@student.bham.ac.uk @Jenny_Hendy 
Attention is one of the most crucial yet elusive skills in sport particularly when athletes are under pressure to perform. Specific cardiac patterns prior to movement onset have been associated with attention. Research, including ours, has established that a performer's heart rate decelerates in the seconds prior to the execution of a target-based motor skill (Cooke et al., 2010, Psychophysiology, 47, 1109-1118). For instance, elite golfers' heart rates drop about twenty beats-per-minute in the five seconds before they strike a putt. Although the heart rate slowing phenomenon is well documented, and appears to be relatively independent of respiratory and/or muscular influences, the specific features of this deceleration profile have yet to be established. Under the premise that increased task difficulty requires greater attention, the current study was designed to improve our understanding of this bradycardia, and furthermore determine which cardiac features correlate strongest with performance, and attentional processes. 36 golfers (18 novices, 18 experts), completed baseline ( $2 \mathrm{~m}$ from hole), and seven counterbalanced 9-ball putting conditions on an indoor putting surface. Conditions were designed to manipulate difficulty, and thus attentional demands, by altering putt distance, hole size, and surface gradient. Heart rate (electrocardiogram) was measured throughout, with performance determined by the number of holed putts in each condition. Separate repeated measure ANOVA's indicated that performance $(P<.001)$ and putt accuracy (mean radial error) $(P<.001)$ were significantly affected by condition. Similarly, main effects of difficulty were seen for several aspects of the cardiac pattern, including peakto-trough amplitude $(P<.05)$, and the rate of the heart rate deceleration $(P<.001)$. Moreover, Fisher-Z scores revealed these changes varied as a function of putt outcome. The rate of heart rate deceleration was found to be the best correlate of performance $(r=.85, P<.001)$. To conclude, heart rate deceleration in the seconds preceding a skilled motor task was affected by variations in task difficulty. Furthermore, successful performance was linked to specific cardiac features. If current results are confirmed, this study should help improve our understanding of the attention-performance relationship. Practically, these findings help validate pre-movement cardiac deceleration as a discreet measure of attention, and provides vital empirical data required to develop future biofeedback training protocols, aimed at improving attentional focus, and thus, performance under pressure.

\section{D2.S4.3(4). Differences in goal-directed self-talk use and functions between individual and team sport athletes and between genders}

\section{ALEXANDER T. LATINJAK ${ }^{1,2}$, YAGO RAMIS ${ }^{3}$ \& MIQUEL TORREGROSSA ${ }^{3}$}

${ }^{1}$ University of Suffolk, ${ }^{2}$ School for Sport and Health Sciences (EUSES), University of Girona, ${ }^{3}$ Universitat Autònoma de Barcelona *Corresponding author: alexander.latinjak@cadscrits.udg.edu @Alex_T_Latinjak

This study focused on goal-directed self-talk (GST), a selfregulatory strategy described as a controlled mental process deliberately employed towards solving a problem or making progress on a task (Latinjak et al., 2014, Psychology of Sport and Exercise, 15, 548-558). Specifically, we aimed to explore differences in GST use and functions between individual and team-sport athletes and between males and females. After obtaining the pertinent institutional ethics approvals, 67 athletes (41 males, 26 females) from individual $(n=19)$ and team ( $n=48)$ sports, answered a questionnaire on GST functions. The questionnaire was based on items from the Automatic Self-talk Questionnaire for Sports (ASTQS, Zourbanos et al., 2009, The Sport Psychologist, 23, 233-251) and assessed the use of GST in training and competition, and the use of GST aimed at cognitive (e.g., to focus) and motivational (e.g., to increase confidence) functions. Regarding results, a two-way (sport type $x$ gender) MANOVA revealed significant effects of Sport on GST use in training $(P<.01)$ and GST use in competition $(P<.05)$, indicating that individual-sport athletes use more GST in both training and competition than team-sport athletes. Further, a two-way MANCOVA, using GST use in training as covariate, yielded significant univariate effects of Sport on cognitive GST in training $(P<.001)$ and motivational GST in training $(P<.05)$, indicating that individual-sport athletes use more cognitive and motivational GST in training than teamsport athletes. Moreover, a two-way MANCOVA, using GST use in competition as covariate, evidenced a significant effect of Gender on cognitive GST in competition $(P<.05)$, indicating that female athletes use more cognitive GST in competition than male athletes. Overall, the results showed that both sport type and gender are of relevance to the design and implementation of self-talk interventions in sports. According to the IMPACT guidelines, in these interventions, self-talk has not only to be matched to the athlete's needs, but also to the coach within (Latinjak et al., 2016, The Sport Psychologist, 30, 189-194), the inner voice that accompanies the athlete during sport participation.

\section{D2.S4.3(5). From Olympic rings to the circus ring: gymnasts' transition experiences into circus}

\section{FLEUR VAN RENS $^{1 *} \&$ EDSON FILHO ${ }^{2}$

\author{
${ }^{1}$ Murdoch University, ${ }^{2}$ University of Central \\ Lancashire \\ *Corresponding author: f.vanrens@murdoch.edu.au
}

Contemporary circus is an art form in which human performers use circus skills to tell a story (Leroux, 2014, Theatre Research in Canada, 35(2), 263-279). Due to the similarities between physical skills in circus and gymnastics, world-renowned circus companies are purposefully recruiting professional athletes for their shows (Rantisi \& Leslie, 2014, Economic Geography, 91(2), 147-164). The aim of this study was to explore the experiences of gymnasts in their journeys towards becoming professional circus artists. With institutional ethics approval, eight (retired) (inter)national level gymnasts (4 male, 4 female) who work(ed) for global circus companies participated in individual semi- structured interviews. On average, the participants were 30.9 years of age, and they performed in aerial and acrobatic circus disciplines such as teeterboard, aerial silks, and Russian cradle. The interviews were transcribed verbatim and analysed using an inductive, semantic, thematic analysis approach. Results indicated 
that participants' motivation to transition to a circus career was sparked by a need to be physically active, and an urge to express themselves. Four participants reported a dislike for the restrictiveness of the sport gymnastics but a love for gymnastic skills as the main motivating factor to pursue a circus career. Resilience was necessary to realise a transition to professional circus, and a barrier that needed to be overcome by two men was parental pressure to choose a 'serious' career upon retirement from sport. Positive transition experiences were characterised by continued opportunities to develop oneself, high levels of perceived autonomy and safety, and social support by the circus community. Challenges within the transition process included adjusting to artistic performance, a perceived drop in competence and confidence, dependence on others while executing skills, and adjusting to the circus culture. Further, all participants experienced identity shifts. Some maintained their athletic identity and added a circus artist identity, whilst others shed their athletic identity in favour of a circus artist identity. This indicates that despite similarities in physical demands, experiences in career transitions to circus resemble aspects of experiences in career transitions out of sport. Collectively, the research findings suggest that the perceived transition quality affects circus artists' mental wellbeing and safety, which in turn appears to affect the likelihood of long-lasting performing careers. It is therefore recommended that the self-determined needs of gymnasts/former athletes are supported in their journey towards becoming circus artists. 


\section{Day 2. Free Communications - Psychology (Session 2)}

\section{D2.S4.4(1). An investigation into the sport ethic of equestrian eventing}

DEBORAH BREWER $^{1 *} \&$ CLARE RHODEN ${ }^{2}$

\author{
${ }^{1}$ Bishop Burton College, ${ }^{2}$ University of Worcester \\ ${ }^{*}$ Corresponding author: \\ deborah.brewer@bishopburton.ac.uk
}

Athletes regularly compete with injury and in pain (Liston et al., 2006, Sport in Society, 9, 388-402) for reasons including dedication to competitive sport, acceptance of pain, and the influential effects of peers and coaches, which can be collectively considered as sport ethic (Coakley, 2009, In Coakley (Ed.), Deviance in Sports (p. 163) New York: McGraw-Hill \& Hughes). This investigation analyses the existence of sport ethic in equestrian eventing. Professional and recreational equestrian event competitors $(n=311)$ completed a questionnaire containing: demographic questions, the Sports Inventory for Pain (SIP) (Meyers et al., 1992, Journal of Sport \& Exercise Psychology, 14, 249-261), the Sports Orientation Questionnaire (SOQ) (Gill \& Deter, 1988, Research Quarterly, 59, 191-202) and the Athletic Identity Measurement Scale (AIMS) (Brewer \& Cornelius, 2001, Academic Athletic Journal, $15,103-113)$. University ethical approval was obtained and the work was supported by British Eventing. Spearman's Rho test demonstrated a positive relationship between SIP Total Coping Response (TCR) subscale and AIMS $\left(r_{s}=0.319, \mathrm{n}=\right.$ $311, P<0.001)$, SOQ competitiveness subscale $\left(r_{s}=0.300, \mathrm{n}=\right.$ $311, P<0.001)$, SOQ goal orientation subscale $\left(r_{s}=0.317, \mathrm{n}=\right.$ $311, P<0.001)$ and SOQ win orientation $\left(r_{s}=0.126, \mathrm{n}=311, P\right.$ $=0.027$ ), indicating the presence of clear sport ethic within equestrian eventing. Further analysis of results demonstrated that a large proportion of riders competed with injury ( $\mathrm{n}=$ $245 ; 78.8 \%)$ and resumed training $(n=214 ; 68.8 \%)$ or competed against medical advice ( $n=136 ; 43.7 \%)$. Independentsample t-test revealed that riders who competed whilst injured scored significantly higher on the SIP subscales for direct coping $(t=3.747, P<0.001)$, cognitive strategies $(t=$ 2.990, $P=0.003)$ and total coping response $(t=4.227, P<$ 0.001 ) indicating they have developed better strategies to deal with pain. Significantly higher SOQ subscales for competitiveness $(t=2.003, P=0.046)$ and win orientation $(t=2.366$, $P=0.019$ ) suggests that those who compete when injured have a stronger competitive nature. This was further supported by the significantly higher score on the AIMS questionnaire ( $t=3.387, P=0.001$ ) confirming that these athletes strongly identify with their sport of equestrian eventing. The findings indicate that a high proportion of riders competed whilst injured, a similar incidence to other sports such as rugby (Liston et al., 2006, Sport in Society, 9, 388-402) and basketball (McKay et al., 2001, British Journal of Sports Medicine, 35, 103-108). Equestrian eventing competitors have a strongly developed sport ethic, with significantly higher scores for riders competing with injury, demonstrating the intertwined effect of sport ethic on athletes' judgments about competing whilst injured.

\author{
D2.S4.4(2). "When do I stop her?" Exploring \\ overuse injuries through the eyes of young \\ gymnasts' parents
}

\section{FRANCESCA CAVALLERIO ${ }^{1 *}$, ROSS WADEY ${ }^{2} \&$ CHRISTOPHER R. D. WAGSTAFF}

\author{
${ }^{1}$ Anglia Ruskin University, ${ }^{2}$ St Mary's University, \\ Twickenham, ${ }^{3}$ University of Portsmouth \\ *Corresponding author: \\ francesca.cavallerio@anglia.ac.uk \\ @youlia83
}

Youth sport parents are perceived as stakeholders in facilitating their children's talent development (Côté, 1999, The Sport Psychologist, 13, 395-417). The literature describes them as providers of experience, not only from a logistical and financial perspective, but also as source of emotional support in time of setbacks (Côté, 1999). Yet, not much is known about the experience of youth sport parents themselves when dealing with their children's injuries. Lally and Kerr (2008, Journal of Applied Sport Psychology, 24, 175-190) suggested gymnasts' parents face doubts concerning their behaviour in dealing with their daughters' daily pain, but these doubts, feelings and the role of parents in relation to sport injuries have received scarce attention. The present study aimed to gain a more in-depth understanding of the experience of young gymnasts' parents when dealing with their daughters' overuse injuries. Data for this study were collected as part of a broader research project (Cavallerio et al., 2016, Psychology of Sport and Exercise, 25, 100-109) that looked at psychosocial factors influencing overuse injuries in gymnastics. After obtaining ethical approval from the University ethical board, a 12-month ethnography was conducted in an elite rhythmic gymnastics club. Twenty-two parents participated in the study through observations, focus groups, informal conversations, and interviews. Following data transcription, emergent themes were identified using thematic analysis. Four main themes resulted from the analysis: (a) empathy (e.g., towards daughters' pain, fatigue, positive and negative moments in gymnastics); (b) worry, mainly related to recognising a lack of knowledge in relation to pain and injuries (e.g., "how long do I use palliatives such as clay and massages, before seeing a doctor?", "what will be the consequences of this constant back pain in the future?"); (c) parent-gymnast relationship, characterised by support, both emotional and logistic, and by communication; and (d) observation of club's environment (e.g., trust in the club and coaches' knowledge, control of caring behaviours, awareness of quality of coach-athlete relationship, reflection on cultural values). These themes illuminate novel aspects in parents' experience of their daughters' overuse injuries, and can help highlight areas where sport stakeholders, such as coaches and managers, can be of support (e.g., by providing information on ways of dealing with pain, explaining the structure of training while in pain). In line with the growing body of research looking at understanding the role of parents in youth sport, this study 
offers a novel resource to positively support youth sport parents in their role as providers of experience for their children.

D2.S4.4(3). Conceptualizing sporting experiences as romantic attachments: a mixed methods exploration of loving the game

\section{ALEX HODGE ${ }^{1,2^{*}}$, EMILY OLIVER ${ }^{1,2}$ \& DAVID ECCLES \\ ${ }^{1}$ Durham University, ${ }^{2}$ Wolfson Research Institute for Health and Wellbeing \\ *Corresponding author: a.c.hodge@durham.ac.uk}

Attachment theory, a dominant theory for understanding human relationships, is broadening in scope through its use in explorations of nonhuman, or non-interpersonal, relationships with places, pets, fictional characters, objects, and deities (Keefer et al., 2014, Social and Personality Psychology Compass, 8(9), 524-535). This mixed method programme of research extends the scope of attachment further by exploring whether (study (1) and how (study 2) athletes attach to their sports. Following university ethical approval, both studies also examined how sporting nonhuman attachments relate to health and wellbeing. In the first quantitative study, participants completed a theoretically-based questionnaire identifying the extent to which their experiences in sport exhibited qualities and characteristics of their interpersonal relationships (e.g., intimacy, security, obsession). Measures of attachment, relatedness, and wellbeing were also completed. Participants $(n=101)$ were predominantly university students $(n=72)$, female $(n=61)$, from North America or Europe $(n=96)$ and involved in team sports $(n=93)$. Results demonstrated that certain features of interpersonal relationships were equally present in sporting nonhuman relationships (e.g., accommodation), while others were more prevalent in sporting relationships (e.g., growth, physical harm) or interpersonal relationships (e.g., communication, intimacy). Multiple regressions controlling for interpersonal associations showed that athletes' sporting nonhuman relationships are differentially associated with a range of positive and negative wellbeing outcomes, similar to interpersonal relationships, but to a lesser degree. In the second study six participants were purposively sampled from the previous study due to the strength of their reported relationships with sport and qualitative analysis was conducted using semi-structured interviews and interpretative phenomenological analysis. Key characteristics of sporting nonhuman relationships emerged as choice, omnipotence or control, and the resultant security they offered the athletes. These results suggest that sporting nonhuman relationships: exist, influence athlete's health and wellbeing, and involve attachments and security. Conceptualising interactions with sport as relationships can inform elements of the entire athletic lifecycle including: what draws certain people to specific sports, why athletes maintain their relationships with sport, and how athletes cope upon the dissolution of a sporting nonhuman relationship.

D2.S4.4(4). Physical abuse in sport: classifying types \& understanding impact

EMMA KAVANAGH*, LORRAINE BROWN \& IAN JONES

Bournemouth University
*Corresponding author: ekavanagh@bournemouth.ac.uk @EmjKavanagh

Empirical data on physical abuse in sport is currently lacking. As a direct consequence, this remains an under-researched area in the sporting domain (Mountjoy et al., 2016, British Journal of Sports Medicine, 1-11). This presentation will explore elite athletes' experience of physical abuse within the coach-athlete relationship and its subsequent impact (immediate and longterm). University ethics was obtained prior to the collection of data. The sample comprised 11 elite athletes ( 6 females, 4 males) between 19 and 35 years of age $(M=27)$ who had competed in the United Kingdom representing England, Wales, and/or Great Britain. Athletes competed across 11 team $(n=8)$ and individual sports $(n=3)$. In-depth semi-structured interviews were completed and analysed inductively using thematic analysis (Braun \& Clark, 2006, Qualitative Research in Psychology, 3 , 77-101). Athletes reported experiencing contact and noncontact physical abuse both directly and also indirectly by witnessing the physical abuse of others within the training or competition environment. Physical abuse was seen to manifest in two primary ways: (1) the presence of physical violence (contact and non-contact violence); and (2) the use of forced physical exertion through the practice of physical punishment, exposure to excessive training methods, and pressure to play while injured or in pain. Coaches were the primary perpetrators of the abuse. The findings suggest that physical abuse can pose a significant threat to an individual's physical, emotional and psychological safety both in the moment it occurs and in the aftermath of the experience. We suggest that sport psychologists need appropriate safeguarding training in order to enhance athlete protection and well-being in sport.

D2.S4.4(5). Experienced sport psychology consultant's engagement in supervision as a tool to stay ethical

\section{LEE-ANN SHARP $^{1 *}$, KEN HODGE $^{2}$, STEVE DANISH ${ }^{3}$ \\ ${ }^{1}$ Ulster University, ${ }^{2}$ University of Otago, ${ }^{3}$ Virginia \\ Commonwealth University \\ *Corresponding author: L.sharp@ulster.ac.uk}

For those sport psychology consultants (SPCs) providing psychological support for athletes competing in pinnacle sports events, the multiple roles, clients, significant time commitments, and emotional highs and lows they may deal with are complex (Anderson, Van Raalte, \& Brewer, 2001, Professional Psychology: Research and Practice, 32, 12). The ethical considerations and challenges faced by SPCs working within the elite sports environment can be numerous and diverse given the range of SPC roles and services provided in frequently non-traditional settings. Researchers have previously cautioned the need for ethical SPCs to exercise even more caution than psychologists in traditional practice settings because of the variety of service delivery structure, multitude of time, and situational challenges they face (Haberl \& Peterson, 2006, Ethics \& Behavior, 16, 25-40). One process of monitoring the ethical practice of SPCs is that of supervision or peer support. The aim of the current investigation was to explore the ethical challenges SPCs have experienced in their applied practice in elite sport; and examine the 
engagement of experienced SPCs in monitoring and supervision of applied practice. Following university research board ethical approval, 10 experienced accredited SPCs (8 male and 2 female; 5 British, 1 Swedish, 1 Austrian/American, 3 American; $\mathrm{M}$ age = 50.44 years; $M$ years consulting experience $=21.67$ years) who provided psychological support to elite athletes competing in major national and international events in a range of team and individual sports. SPCs participated in individual semi-structured, face-to-face interviews with the primary investigator that lasted between $70 \mathrm{~min}$ to $90 \mathrm{~min}$ and yielded 188 single-spaced pages of data. Following an inductive thematic content analysis approach (Weber, 1990, Basic content analyses. London: Sage), two categories emerged within SPC discussions on the ethical challenges they face, these included, challenges to boundaries; and communication issues. Additionally, SPCs perceived supervision as essential for applied practice as it enables SPCs to monitor their practice, get to know themselves, care for themselves and monitor their boundaries. Four sub-categories emerged in support of this, these included; supervision is an essential component of applied practice; supervision enables SPCs to monitor boundaries of applied practice; supervision helps SPCs to feel supported in their applied practice; and supervision aids SPCs to get to know themselves and care for themselves. The place of supervision and peer support should be considered by all practitioners working within applied sport psychology. 


\section{Day 2. Free Communications - Sport and Performance}

\author{
D2.S4.5(1). Match demands in elite women's \\ English Premiership rugby union
}

\author{
EDDIE BRADLEY ${ }^{1,2^{*}}$, PHIL SHARPE ${ }^{2}$, BOB HOGG $^{1}$ \& \\ DAVID ARCHER ${ }^{1}$ \\ ${ }^{1}$ University of Sunderland, ${ }^{2}$ Darlington Mowden Park \\ Sharks \\ ${ }^{*}$ Corresponding author: \\ eddie.bradley@sunderland.ac.uk \\ @DrEddieBradley
}

Despite the growing popularity of women's rugby union over the past decade, little attention has been given to understanding the physical and technical demands specific to the sport. The aim of the study was to determine the match demands players are required to achieve during an elite level season and to identify if positional differences exist. Global positioning system (GPS) data were collected from one English Women's Premiership rugby union team over the course of the 2016/17 season. A total of 24 players in 8 games wore Catapult Minimax S4 units and data was obtained from players who had completed $>60$ min per game (total player games $=$ 78). Players were classified according to specific position groups (front, second, back row, scrum half, inside and outside backs). Total and relative distances were calculated at the following movement intensities; 0-6 km/h (walking), 6.1-12 $\mathrm{km} / \mathrm{h}$ (jogging), $12.1-14 \mathrm{~km} / \mathrm{h}$ (slow running), 14.1-16 km/h (medium running), $16.1-18 \mathrm{~km} / \mathrm{h}$ (fast running), $>18.1 \mathrm{~km} / \mathrm{h}$ (sprinting). Maximum velocity and player loads were calculated as an indication of overall player workload. Comparisons of mean values were made between playing positions with statistical significance determined using an independent measures ANOVA in SPSS v23. Mean total match distance was greatest for the second row $(5185 \mathrm{~m} \pm$ 1570) and least for the outside backs (3778 $m \pm 1281$ ), with similar patterns for relative distances, although no position was significantly different from any other. Outside and inside backs covered a significantly greater distance at fast running $(129 \mathrm{~m} \pm 88 ; 138 \mathrm{~m} \pm 100)$ and sprinting $(90 \mathrm{~m} \pm 103 ; 76 \mathrm{~m} \pm$ 88) speeds, whereas the second row and scrum half covered significantly greater distances walking (2356 m $\pm 667 ; 2477 \mathrm{~m}$ $\pm 380)$ and jogging (1798 $\mathrm{m} \pm 530 ; 1716 \mathrm{~m} \pm 459)$ and the front row spent the greatest overall distance walking (2488 m \pm 629 ). Outside backs produced a significantly greater maximum velocity than all other positions $(24.4 \mathrm{~km} / \mathrm{h} \pm 1.95)$, inside backs were significantly faster than the forwards and scrum half $(22.9 \mathrm{~km} / \mathrm{h} \pm 2.28)$, with the front row having the slowest maximum velocity $(20.3 \mathrm{~km} / \mathrm{h} \pm 2.3)$. Mean player load was highest in the scrum half $(559 \mathrm{AU} \pm 101)$ and second row $(531 \mathrm{AU} \pm 140)$ and these were significantly higher than the outside backs. These findings indicate the demands placed on elite female rugby players are position specific and are different from male counterparts. These demands should be taken into account when setting training drills and match strategies.

\begin{abstract}
D2.S4.5(2). An examination of a modified Yo-Yo test to measure intermittent running performance in rugby players
\end{abstract}

\author{
NICK DOBBIN ${ }^{1,2^{*}}$, SAMANTHA MOSS ${ }^{1}$, JAMIE HIGHTON ${ }^{1}$ \\ \& CRAIG TWIST $^{1}$
}
${ }^{1}$ Department of Sport and Exercise Science, University of Chester, ${ }^{2}$ Rugby Football League
${ }^{*}$ Corresponding author: n.dobbin@chester.ac.uk @Nick_Dobbin

High intensity intermittent running capacity is an essential quality for rugby league players that can be assessed using the Yo-Yo Intermittent Recovery Test (Yo-Yo IR1; Bangsbo, laia, \& Krustrup, 2008, Sports Medicine, 38, 37-51). However, that a player's running during matchplay is frequently punctuated by having to get up from the floor after a tackle means the traditional Yo-Yo IR1 does not account for the increased demands associated with the tackle (Mullen, Highton, \& Twist, 2016, International Journal of Sports Physiology and Performance, 10, 746-753). Accordingly, this study examined the influence of altering the start position before each shuttle on the physiological, perceptual and external responses during the Yo-Yo IR1. With institutional ethics approval, 17 male rugby players (mean \pm SD age $=20.4 \pm$ 1.2 years, stature $=182.6 \pm 5.7 \mathrm{~cm}$, body mass $=83.7 \pm$ $9.5 \mathrm{~kg}$ ) completed two trials of the Yo-Yo IR1 one week apart using a randomized cross over design and shuttles started in either a standing or prone position. External loads were measured using microtechnology alongside expired air, heart rate, rating of perceived exertion (RPE) and blood lactate concentration $\left([\mathrm{La}]_{\mathrm{b}}\right)$. Values are presented as mean \pm SD with differences between trials reported using effect sizes $(E S) \pm 90 \%$ confidence interval $(90 \% \mathrm{Cl})$ and magnitude-based inferences. Total distance was most likely lower during the prone compared to standing Yo-Yo IR1 $(619 \pm 160$ cf. $964 \pm 222 \mathrm{~m}$; ES \pm $90 \% \mathrm{Cl}:-1.87 \pm 0.19)$. Peak PlayerLoad ${ }^{\mathrm{TM}}(14.6 \pm 1.4$ cf. $13.9 \pm 0.9 \mathrm{AU} / \mathrm{min}$; $\mathrm{ES} \pm 90 \% \mathrm{Cl}: 0.70 \pm 0.42)$, acceleration at $4-20 \mathrm{~m} / \mathrm{s}(3.0 \pm 0.9 \mathrm{cf} .2 .4 \pm 0.6 \mathrm{~m} / \mathrm{min}$; ES $\pm 90 \%$ $\mathrm{Cl}: 0.79 \pm 0.44)$ and high metabolic power $(5.3 \pm 1.2 \mathrm{cf}$. $3.5 \pm 0.9>20 \mathrm{~W} / \mathrm{kg} / \mathrm{min}$; ES $\pm 90 \% \mathrm{Cl}: 1.80 \pm 0.32$ ) were very to most likely higher during the prone Yo-Yo IR1. Heart rate was possibly lower $(195 \pm 7$ cf. $197 \pm$ $8 \mathrm{~b} / \mathrm{min}$; ES $\pm 90 \% \mathrm{Cl}:-0.26 \pm 0.25)$, RPE very likely higher $(18.2 \pm 1.5$ cf. $17.1 \pm 1.6 \mathrm{AU} ; \mathrm{ES} \pm 90 \% \mathrm{Cl}: 0.63 \pm 0.41)$ and $\checkmark \mathrm{O}_{2 \text { peak }}$ likely higher $(50.2 \pm 4.5 \mathrm{cf} .48 .7 \pm 3.8 \mathrm{ml} / \mathrm{kg} / \mathrm{min}$; $\mathrm{ES} \pm 90 \% \mathrm{Cl}:=0.37 \pm 0.39$ ) during the prone compared to standing Yo-Yo IR1. Starting shuttles in the prone rather than standing position alters the physiological, perceptual and external responses to the Yo-Yo IR1 that emphasises a player's ability to perform metabolically demanding actions. 
D2.S4.5(3). Within and between-session reliability of jump performance in elite rugby union players

ADAM GRAINGER ${ }^{1 *}$, PAUL COMFORT ${ }^{2}$, JOHN MCMAHON ${ }^{2} \&$ PAUL JONES ${ }^{2}$

\author{
${ }^{1}$ University College Dublin, ${ }^{2}$ University of Salford \\ *Corresponding author: adam.grainger@ucd.ie \\ @atgrainger
}

Jump testing is commonly used within elite field sport settings to measure fatigue. This study assessed the within and between session reliability of three jump-tests, including countermovement jump (CMJ), squat jump (SJ) and single leg drop jumps (left $=$ SLDJ-L; right $=$ SLDJ-R). Eight elite rugby union players (age $21.0 \pm 4.4$ years, height $185.0 \pm 8.0 \mathrm{~cm}$, mass $90.0 \pm 8.2 \mathrm{~kg}$ ) completed two trials of each jump, on three occasions, using an OptoJump assessing jump height via a flight time calculation for the CMJ and SJ, and contact time for the SLDJ. Reliability within session was determined via intraclass correlation coefficients (ICC) and between session reliability was determined using both ICC and repeated measures ANOVA with Bonferroni post hoc analysis. Within session reliability was high for the $\mathrm{CMJ}$ and SJ (ICC $=0.938$; ICC $=0.954$ respectively), moderate for the SLDJ$\mathrm{L}(\mathrm{ICC}=0.759)$ and low for the SLDJ-R (ICC = 0.445). Between sessions CMJ and SJ showed high reliability $(C M J$ ICC $=0.906$; SJ ICC $=0.866)$, yet significant differences were noted between testing days 1 and 2 for $C M J(P<0.05, E S=0.329)$ and small non-significant differences observed between days for $S J$ (ES = 0.171). Unilateral jumps showed varying reliability (SLDJ-L ICC = 0.875; SLDJ-R ICC $=0.759$ ) between sessions, with a small and non-significant $(P>0.05$, SLDJ-L ES $=0.321$, SLDJ-R L ES $=0.199$ ) difference between days. This study illustrates that the CMJ represents high reliability both within and between session in comparison to the SJ and SLDJ, and that CMJ demonstrates the lowest smallest detectable difference $(S D D=1.7 \%$ ) between sessions compared to the SJ (SDD = 3.0\%), SLDJ-L (SDD = $4.8 \%$ ) and SLDJ-R (SDD = 7.9\%). Despite a potential learning effect existing for $\mathrm{CMJ}$ in this study, these results are consistent with other research (Bosquet, Berryman, \& Dupuy, 2009; Glatthorn et al., 2011), with the higher reliability reported for bilateral jumps when compared to unilateral jumps likely due to a bilateral jump requiring less variation in technique adopted. Further research investigating unilateral jumps, with a standardised technique implemented across all participants is needed as findings from this research suggest that unilateral jumps should not be used as a performance indicator. Additionally, important findings from this study indicate that practitioners should consider a change in jump height of $\geq 1.7 \%$ as meaningful when assessing elite rugby union players performing CMJ.

\section{D2.S4.5(4). Integrated internal and external} training loads for field based measures of fitness in rugby union

\section{RICHARD TAYLOR*, DAJO SANDERS \& IBRAHIM AKUBAT}

\author{
Newman Univesity \\ *Corresponding author: tayl421@newman.ac.uk \\ @DickiTaylor
}

In Rugby Union, weekly competitive matches make regular fitness testing challenging during the in-season period. Previous studies have demonstrated the integration of training load (TL) data shows relationships with indices of aerobic fitness in soccer \& hurling, making inferences of fitness possible from routinely collected training data (Akubat et al., 2014, International Journal of Sports Physiology and Performances, 9, 457-462). The aim of this study was to assess the relationships between integrated TL ratios and fitness in academy rugby union players with different training exercises common in rugby union. With institutional ethics approval, twelve academy rugby players from a National League One club competing in the Association of Colleges Elite League agreed to participate in the study (aged; $17.7 \pm 0.8$ years, height; $179.4 \pm 6.3 \mathrm{~cm}$, body mass $83.3 \pm 9.7 \mathrm{~kg}, \mathrm{VO}_{2 \max } 46.2 \pm 3.8$ $\left.\mathrm{mL} . \mathrm{kg}^{-1} \cdot \mathrm{min}^{-1}\right)$. Participants completed an incremental treadmill test for $\mathrm{VO}_{2 \max }$, velocity at blood lactate concentrations of $2 \mathrm{mmol} \cdot \mathrm{L}^{-1}$ (vLT) $4 \mathrm{mmol} \cdot \mathrm{L}^{-1}$ (vOBLA) and the heart rate-blood lactate profile for the calculation of individualised trainingimpulse (iTRIMP). The following week players engaged in three field-based training protocols: $760 \mathrm{~m}$ continuous shuttle running (CSR; $20 \mathrm{~m}$ shuttle run at $9 \mathrm{~km} \cdot \mathrm{h}^{-1}$ ), sprint interval training (SIT; $6 \mathrm{x}$ $6 \mathrm{~s}$ sprint with $54 \mathrm{~s}$ recovery) and a small sided game (SSG; $10 \mathrm{~min}$ on a $39 \times 51 \mathrm{~m}$ pitch). Internal TL was calculated using iTRIMP and external TL measures were collected using a Microelectromechanical systems device (10 Hz GPS, $100 \mathrm{~Hz}$ Accelerometer) included total distance (TD), PlayerLoad (PL), metabolic power (MP), very high-speed distance $>18 \mathrm{~km} \cdot \mathrm{h}^{-1}$ (VHSD), high-speed distance $>15 \mathrm{~km} \cdot \mathrm{h}^{-1}$ (HSD) and individualised high-speed distance based on each player's vOBLA (iHSD). The external TL was divided by the internal TL to form TD:TTRIMP, PL:iTRIMP, MP:iTRIMP, iSHD:iTRIMP, HSD:TTRIMP, VHSD:TTRIMP ratios. Correlation analysis assessed the relationships between fitness measures and the ratios from the CSR, SIT and SSG. All ratios demonstrated a large to very large associations with vOBLA (Rho=0.64-0.76) and vLT (Rho=0.63-0.71) across training drills. Associations with $\mathrm{VO}_{2 \max }$ were small to moderate ( $\mathrm{Rho}=$ $0.20-0.43$ ). The results of this study suggest that a range of integrated external:internal TL ratios can be used for the assessment of aerobic fitness in rugby union in a variety of training modalities. Furthermore, ratios using simpler measures of external load such as TD show similar relationships to more complex measures such as MP and PL.

\section{D2.S4.5(5). Exercise-induced muscle damage and changes in resting metabolic rate after intermittent running with and without physical contact}

\section{CRAIG TWIST ${ }^{1 *}$, JOSHUA LEE $^{1,2}$ \& JAMIE HIGHTON \\ ${ }^{1}$ University of Chester, ${ }^{2}$ Catapult Sports \\ ${ }^{*}$ Corresponding author: c.twist@chester.ac.uk @craig_twist}

Collision sport athletes experience tissue damage caused by mechanical strain from repeated muscle contraction and blunt force trauma from physical contact (Oxendale et al., 2016, International Journal of Sports Physiology and Performance, 11, 515-521). This study examined symptoms of exercise-induced muscle damage and resting metabolic rate $24 \mathrm{~h}$ after intermittent running with or without physical contact. With institutional ethics approval, nine male team sport players (mean $\pm s$ 
age $22.8 \pm 2.9$ years; stature $1.80 \pm 0.06 \mathrm{~m}$; body mass $80.0 \pm 5.4$ $\mathrm{kg}$ ) completed two trials of a $2 \times 23$ min intermittent running protocol with and without physical contact. Trials were performed one week apart using a randomized crossover design. Upper- and lower-body muscle function, creatine kinase activity $(\mathrm{CK})$ and resting metabolic rate were measured before and $24 \mathrm{~h}$ after both trials. Movement, heart rate and session rating of perceived exertion (sRPE) were measured during the protocol. Values are presented as mean $\pm s$ with differences between trials reported using the effect size (ES) $\pm 90 \%$ confidence interval $(90 \% \mathrm{Cl})$ and magnitude-based inferences. Relative distance $(105 \pm 4.5$ cf. $98 \pm 2.3 \mathrm{~m} / \mathrm{min}$; ES $\pm 90 \% \mathrm{Cl}: 2.86 \pm 0.81$, most likely), change in peak sprint speed ( $7 \pm 9$ cf. $2 \pm 4 \%$; ES \pm $90 \% \mathrm{Cl}: 0.57 \pm 0.72$, likely), mean heart rate (163 $\pm 7 \mathrm{cf} .157 \pm 11$ b/min; ES $\pm 90 \% \mathrm{Cl}: 0.49 \pm 0.35$, likely) and sRPE (7.4 \pm 1.3 cf. 4.7 $\pm 2.0 \mathrm{AU} ; \mathrm{ES} \pm 90 \% \mathrm{Cl}: 1.25 \pm 0.34$, most likely) were all higher during the contact trial. Upper body force loss was greater after the contact trial $(13 \pm 11 \mathrm{cf}$. $2 \pm 8 \%$; ES $\pm 90 \% \mathrm{Cl}$ : $0.45 \pm 0.54$, likely), while lower body force loss was unclear between trials ( $5 \pm 5$ cf. $5 \pm 6 \%$ : $\mathrm{ES} \pm 90 \% \mathrm{Cl}:-0.39 \pm 1.28)$. CK increased after the contact (179 $\pm 49 \%$; $\mathrm{ES} \pm 90 \% \mathrm{Cl}: 1.41 \pm 0.63$, most likely) and non-contact trials ( $191 \pm 96 \%$; ES $\pm 90 \% \mathrm{Cl}$ : $0.82 \pm 0.60$, very likely). Resting metabolic rate was increased after the contact $(+142 \pm 217 \mathrm{kcal} /$ day; $\mathrm{ES} \pm 90 \% \mathrm{Cl}: 0.50 \pm 0.47$, likely) and noncontact trials $(+157 \pm 149 \mathrm{kcal} / \mathrm{day} ; \mathrm{ES} \pm 90 \% \mathrm{Cl}: 0.67 \pm 0.37$, very likely). Intermittent running with or without contact causes exercise-induced muscle damage and small to moderate increases in resting metabolic rate. Practitioners can use these data to inform the nutritional and recovery practices of collision sport athletes. 


\section{Day 2. 5 slides in 5 min Free Communications - Biomechanics and Motor Behaviour / Physiology and Nutrition}

\author{
D2.S1.1(1). Is relative economy explained by \\ sagittal plane trunk movements in back, back/front \\ and head loading?
}

SEAN HUDSON ${ }^{1 *}$, CARLTON COOKE $^{1}$, SIMEON DAVIES ${ }^{2}$, SACHA-JANE WEST ${ }^{2}$, RAEEQ GAMIELDIEN ${ }^{2}$, CHRIS LOW $^{3}$ \& RAY LLOYD ${ }^{1}$

\section{${ }^{1}$ Leeds Trinity University, ${ }^{2}$ Cape Peninsula University of Technology, ${ }^{3}$ Leeds Beckett University \\ ${ }^{*}$ Corresponding author: s.hudson@leedstrinity.ac.uk @sean_hudson_}

Back-loading is very economical with light loads (up to $12 \mathrm{~kg}$ ) (Abe et al., 2004, Applied Ergonomics, 35, 329-335). With heavier loads $(25.6 \mathrm{~kg})$, back/front-loading appears to be more economical (Lloyd \& Cooke, 2000, European Journal Applied Physiology, 81, 486-492). This is, perhaps, because it allows for greater trunk range of motion (RoM), which is associated with a reduced requirement to generate propulsive force (Lloyd \& Cooke, 2000, Ergonomics, 43, 1331-1341). Light loads on the back shouldn't constrain posture as much as heavy loads and variations in back-loading economy may be explained by contributions to forward momentum afforded by trunk RoM. Conversely, head-loading requires a constrained, upright posture to balance the load. This study assessed the association of sagittal plane trunk movement and load carriage economy by comparing three methods that constrain posture differently. With institutional ethics approval, eighteen female volunteers (age $23 \pm 3.8$ years, mass $61.1 \pm 10.7 \mathrm{~kg}$, stature $158.9 \pm 8.1 \mathrm{~cm}$, mean $+\mathrm{sd}$ ) performed separate walking trials at $3 \mathrm{~km} \cdot \mathrm{h}^{-1}$ for back, back/front and head-loading. Each trial involved $7 \times 4$ min periods of walking, separated by two minutes rest. The initial stage involved unloaded walking, followed by walking with 3, 6, 9, 12, 15 and $20 \mathrm{~kg}$. Relative economy was assessed using the Extra Load Index (ELI = oxygen consumption loaded per total kg / oxygen consumption unloaded per kg). Forward lean was the angle of the trunk from horizontal. Trunk RoM was the change in trunk angle from heel-strike to toe-off in each step. There were no differences in relative load carriage economy between methods $(E L I=0.93 \pm 0.08,0.94 \pm 0.06$ and 0.95 \pm 0.11 , for back, back/front and head-loading, respectively; $P=$ 0.483 ), despite significant differences in forward lean (with all load mass combined Back $=6 \pm 2.7^{\circ}$, Back/front $=1.6 \pm 2.0^{\circ}$, Head $\left.=-3.5 \pm 2.8^{\circ} ; P=0.001\right)$ and trunk RoM between methods $(P=0.023)$. Furthermore, there were no relationships between relative economy and forward lean (strongest, $r=$ -0.535 ) or relative economy and trunk RoM (strongest, $r=$ -0.507). Based on mean data, load carriage economy is not explained by sagittal plane trunk movements. Analysis of individual responses may shed more light on the issue. It's likely that a number of factors combine to influence an individual's load carriage economy rather than there be a single set of generalizable factors for all individuals.

\author{
D2.S1.1(2). Biomechanical influences in injury \\ rates of United States community rugby-7s: contact \\ versus non-contact injuries
}

VICTOR LOPEZ JR ${ }^{1,2^{*}}$, RICHARD MA ${ }^{2,3}$, MERYLE G. WEINSTEIN ${ }^{1,4}$, PATRIA A. HUME ${ }^{2}$, ROBERT C. CANTU ${ }^{5,6}$, CHRISTIAN VICTORIA ${ }^{1,7}$, SAMUEL Y. HALEEM ${ }^{1,8}$, JESSICA F. DELALLO ${ }^{1,9}$ \& ANSWORTH A. ALLEN ${ }^{10,11}$

${ }^{1}$ Rugby Research and Injury Prevention Group, Hospital for Special Surgery, ${ }^{2}$ Auckland University of Technology, Sports Performance Research Institute New Zealand, ${ }^{3}$ University of Missouri, Missouri Orthopaedic Institute \& Comparative Orthopaedic Laboratory, ${ }^{4}$ New York University, Steinhardt School of Culture, Education and Human Development, ${ }^{5}$ Emerson Hospital, ${ }^{6}$ World Rugby, Independent Concussion Group, ${ }^{7}$ New York University, Global Institute of Public Health, ${ }^{8}$ City University of New York, The City College, ${ }^{9}$ Tulane School of Medicine, ${ }^{10}$ Hospital for Special Surgery, ${ }^{11}$ National Basketball Association, New York Knickerbockers *Corresponding author: drvictorlopezjr@gmail.com @rripg

Rugby-7s is growing in popularity, however there is a comparative lack of injury data. (Fuller et al., 2010, Clinical Journal of Sports Medicine, 20, 179-184; Lopez et al., 2012, American Journal of Sports Medicine, 40, 179-184). Rugby-7s has increased risk with speed, ball-in-play, sudden starts and stops (Fuller et al., 2010) and demand in play with universal roles of playing positions and associated injury, especially between "backs" and their potential velocity with contact, found higher than "forwards" (Fuller et al., 2010; Lopez et al., 2012). The aim of this study was to report injury incidence of contact and non-contact causes of U.S. Rugby-7s injuries. Prospective injury incidence (per 1000 player-hour (ph)) and mechanism were captured using the Rugby Injury Survey \& Evaluation (RISE) methodology (Lopez et al., 2012). Time-loss injuries occurred when players did not return to play the day of injury. Medical attention injuries occurred with no player absence from play. Severity was defined as days absent before return to training/competition (including post tournament). With institutional ethics approval, U19 to elite amateur players in U.S. Rugby tournaments (2010-2014) were recruited. A total of 24,418 U.S. players (Men $=17,770$; Women $=6.768$; age: $13-49$ years $)$ in 57 tournaments (72 days), 4,448 matches (Men $=3,238$; Women $=1,261$ ), and 2,038 teams (Men $=1,474$; Women $=564$ ) were included. Contact injuries $(54 \% ; n=823)$ occurred more often than non-contact $(46 \% ; \mathrm{n}=697)(P<0.001)$. Medical attention injuries occurred more often (contact $=30.4 / 1000$ ph; noncontact $=29.2 / 1000 \mathrm{ph} ; P<0.001)$ than time-loss injuries (contact $=23.8 / 1000 \mathrm{ph}$; non-contact $=16.7 / 1000 \mathrm{ph} ; P<$ 0.001). No differences were found between non-contact 
injuries and genders (men $=48.1 / 1000 \mathrm{ph}$; women $=46.95 /$ $1000 \mathrm{ph} ; P=0.621$ ). Days absent were frequent among $63 \%$ with follow-up data (contact $=34$ days; Cl: 26.5-41.3; noncontact $=32$ days; $C l: 26.6-39.3)$. Most injuries were new acute match injuries (94\%) (contact 57\%; non-contact 43\%; $P<$ 0.001). Most injuries occurred during tackling (contact $=$ $77 \%$ ), followed by running/open play (non-contact $=32 \%$ ). Ligament injuries were most common among both types of injuries (non-contact $=23 \%$; contact $=30 \%$ ). Similar rates of non-contact injuries were found in both field types (grass = $46.1 / 1000 \mathrm{ph}$; artificial $=52.4 / 1000 \mathrm{ph} ; P=0.467)$. Due to the frequency of contact injuries found in the sport, tackling techniques must be evaluated as an area for injury prevention in the developing U.S. Rugby 7s population. The cause of noncontact injuries with increased open-play needs attention for injury prevention and management.

\section{D2.S1.1(3). Pacing strategy, performance} parameters and kinematical analysis of club level adolescent rowers in a simulated rowing ergometer race

APOSTOLOS S. THEODOROU ${ }^{1 *}$, DIMITRIOS KOURBETIS ${ }^{2}$, ELENI DIMAKOPOULOU ${ }^{1}$, VASSILIOS PANOUTSAKOPOULOS ${ }^{2}$, BARNEY WAINWRIGHT ${ }^{3}$, SOKRATIS KALOUPSIS ${ }^{1}$, \& IRAKLIS A. KOLLIAS ${ }^{2}$

\section{${ }^{1}$ National and Kapodistrian University of Athens, \\ ${ }^{2}$ Aristotle University of Thessaloniki, ${ }^{3}$ Leeds Beckett University \\ *Corresponding author: aptheod@phed.uoa.gr}

Rowing ergometers are considered to simulate the rowing movement and are a widely used mode of training in rowing. Pacing strategies have significant effects on performance and it is suggested that variations in pace may be detrimental to performance (Garland, 2005, British Journal of Sports Medicine, 39, 39-42). Previous research suggested that biomechanical differences exist between elite and junior rowers at predefined paces (Cerne, Kamnik, Vesnicer, Gros, \& Munih, 2013, Human Movement Science, 32, 691-707). The aim of the study was to detect possible performance and biomechanical differences in club level male adolescent rowers in separate sectors of a simulated $2000-\mathrm{m}$ race on a rowing ergometer. With institutional ethics approval, 25 male post-pubertal adolescent club level rowers (mean age: $15.1 \pm 0.7$ years; stature: $1.79 \pm 0.05 \mathrm{~m}$; body mass: $68.3 \pm 6.4$ $\mathrm{kg}$ ) with similar training history were examined. Participants performed an all-out 2000-m simulated race on a Concept2 Model D (Concept2 Inc., Morrisville, VT) rowing ergometer. Performance parameters for each $500-\mathrm{m}$ sector of the race were retrieved using the Concept2 Utility 6.94 software. The angular kinematic parameters of the lower extremity joints were extracted with a 2D-DLT analysis that was conducted using the KAPA-MOTION v.15 software (Kapa-Invent, Orsay, France) after recording the participants with a Casio ExilimPro-EX-F1 (Casio Computer Co. Ltd, Shibuya, Japan) video camera (sampling frequency: $30 \mathrm{fps}$ ). Differences were checked with one-way repeated measures ANOVA with 4 levels of sector, using the SPSS 10.0.1 software (SPSS Inc., Chicago, IL, USA). Results revealed that the time to complete the 500-m sectors was significantly $(P<.05)$ slower after the first sector. The same trend was observed for handle pull velocity, peak stroke force, power and work and body center of mass horizontal displacement. Stroke rate was significantly lower in the middle sections than the first and last. Ankle range of motion was significantly smaller at the last sector, while the knee angle at the initiation of the pull was more flexed after the first sector. Despite the differences in body configuration from sector to sector, no differences were observed for the ankle and knee peak angular velocity. The findings suggest that performance during a simulated 2000- $\mathrm{m}$ race is related to the capability to generate power during the stroke rather than changes in lower limb kinematics, indicating that muscle coordination patterns are stable throughout the 2000-m simulated race (Turpin, Guevel, Durand, \& Hug, 2011, European Journal of Applied Physiology, 111, 3017-3029).

\section{D2.S1.1(4). Optimal timing of low dose caffeine ingestion in trained cyclists}

\section{ANDREW D. DAVENPORT ${ }^{1}$, CATHERINE R. MIKUS ${ }^{2}$, NIMA ALAMDARI $^{2}$ \& FRANCIS B. STEPHENS ${ }^{1 *}$}

\section{${ }^{1}$ University of Exeter, ${ }^{2}$ Beachbody LLC \\ *Corresponding author: f.b.stephens@exeter.ac.uk}

The use of caffeine to improve physical and cognitive performance is widespread amongst athletes (Del Coso, Muñoz, \& Muñoz-Guerra, 2011, Applied Physiology, Nutrition and Metabolism, 36(4), 555-561). Low-dose caffeine $(<3 \mathrm{mg}$. $\mathrm{kg}^{-1}$ body mass, $\sim 200 \mathrm{mg}$ ) has been shown to provide ergogenic effects without the negative side-effects often reported with higher doses (Spriet, 2014, Sports Medicine, 44, S175-S184). However, research on the most efficacious time to consume caffeine is limited. The aim of this study was to assess the effect of timing of ingestion of a commercially available caffeine drink (Energize ${ }^{T M}$, Beachbody, LLC, USA), on exercise performance and perception of effort. With institutional ethics approval, 13 (12 male, 1 female) cyclists $\left(27 \pm 2\right.$ years; $70.8 \pm 2.2 \mathrm{~kg} ; \mathrm{VO}_{2} \max : 64.5 \pm 1.4 \mathrm{ml}$. $\mathrm{kg}^{-1} \cdot \mathrm{min}^{-1}( \pm$ SEM $)$ ) completed four experimental visits after an overnight fast, separated by $\geq 1$ week. Each visit consisted of participants resting (REST) for $30 \mathrm{~min}$ before performing 30 min of steady state (SS) exercise on a cycle ergometer at around $80 \% \mathrm{VO}_{2} \max (243 \pm 8 \mathrm{~W}$ ) followed by a 15 -min timetrial (TT), with perceived exertion (RPE) measured every 10 min during SS and every 5 min during $\Pi$. In addition, before the REST, SS, and TT phases, whilst seated on the ergometer, participants consumed a $473 \mathrm{ml}$ test drink (i.e. three $473 \mathrm{ml}$ drinks per visit). The drinks contained $200 \mathrm{mg}$ caffeine (CAFF) or a caffeine-free, taste-, colour-, and calorie-matched placebo, and were administered in a double-blind, random order such that each visit tested the timing of caffeine ingestion as follows: CAFF before REST, placebo before SS and TT (CAFF-REST visit); CAFF before SS, placebo before REST and $\Pi$ (CAFF-SS); CAFF before $\Pi$, placebo before REST and SS (CAFF-TT); Placebo before REST, SS, and TT (PLA). Differences in $\Pi$ performance and RPE were identified using a repeated measures one- and two-way analysis of variance, respectively. Total work performed during $T T$ in CAFF-REST was greater than PLA $(3.53 \pm 0.14$ vs. $3.36 \pm$ $0.12 \mathrm{~kJ}^{\mathrm{kg}}{ }^{-1}$ body mass; $\left.P=0.005\right)$, and not CAFF-T (3.39 
$\pm 0.13 \mathrm{~kJ} . \mathrm{kg}^{-1}$ - or CAFF-SS $\left(3.44 \pm 0.14 \mathrm{~kJ} . \mathrm{kg}^{-1} ; P=0.231\right)$, which were similar to PLA. Furthermore, there was a treatment effect $(P<0.05)$ for RPE during SS, with the lowest values seen in the CAFF-REST condition compared to PLA and CAFF-SS combined $(P<0.05)$, but no differences were seen during $T T$ when more work was performed. In summary, ingestion of CAFF 30 min before intense SS exercise reduced RPE and improved subsequent 15-min TT performance, whereas ingestion immediately before SS or TT had no effect. This suggests that low-dose caffeine should be ingested $\geq 60$ min before expected fatigue for optimal performance.

D2.S1.1(5). One week L-Arginine supplementation did not improve $200 \mathrm{~m}$ swimming time in trained swimmers

\section{OZCAN ESEN* \& CERI NICHOLAS}

\section{University of Chester \\ *Corresponding author: ozcanesen@hotmail.com @ozcan_esen}

L-arginine (ARG) is semi-essential amino acid and important for the biosynthesis of nitric oxide (NO), an essential substance that increases blood flow (Wu \& Meininger, 2000, The Journal of nutrition, 130(11), 2626-2629). Research on healthy humans and athletic populations are still inconsistent and no studies exist to date which have examined ARG supplementation on swimmers. Parallel and non-weight-bearing body position in swimming provide more blood pumping to muscles and therefore more effective circulatory system (Maglischo \& Brennan, 1985, Swim for the health of it. Mayfield). Hence, increased NO level may be more effective on swimming performance. The present study aimed to determine the effect of ARG supplementation on $200 \mathrm{~m}$ swimming performance. Ethical approval was obtained from the Faculty of Applied and Health Sciences ethics committee at the University of Chester. All subjects trained a minimum of 3 times per week to maintain their training condition on a regular, planned basis, for health, but not for a specific event. In a randomised, cross-over, double-blind design, eight trained male swimmers firstly performed a baseline time-trial (TT) with no supplements. The next 2 trials, they consumed either: $8 \mathrm{~g}$ of ARG or PLA capsules (8 capsules of 1 gram each) over 7-days. Following supplementation period, participant completed a 200-m TT. Blood lactate concentration (BLa) was measured at rest, before and after $\Pi$ via blood lactate analyser. ARG supplementation had an $80 \%$ likely trivial effect $(-0.0 \pm 0.14 \mathrm{~s} ; \mathrm{d}=0.13)$ on $200 \mathrm{~m}$ $\pi$ performance $(146.02 \pm 10.35 \mathrm{sec} ; 147.59 \pm 10.86 \mathrm{sec}$ in ARG and PLA, respectively), an $82 \%$ likely trivial $(-0.20 \pm$ $0.24 \mathrm{~s} ; \mathrm{d}=0.04)$ in first $100 \mathrm{~m}$, and unclear effect $(-0.06 \pm$ $0.14 \mathrm{~s} ; \mathrm{d}=0.20$ ) on second $100 \mathrm{~m}$. There was a $78 \%$ likely moderate $(-1.17 \pm 0.68 \mathrm{mmol} / \mathrm{L} ; \mathrm{d}=0.49)$ increase in BLa $(13.13 \pm 1.77 \mathrm{mmol} / \mathrm{L} ; 12.15 \pm 2.67 \mathrm{mmol} / \mathrm{L}$ in ARG and PLA, respectively). ARG supplementation did not provide considerable benefits for $200 \mathrm{~m}$ swimming performance. Despite expectation was a decrease, there was a increase in BLa after ARG. This may relate to increase mean power output as per other modes of sprint exercise. Further studies should be performed on longer distance due to its more aerobic condition. ARG-depended pathway requires and uses oxygen for NO synthesis. Therefore, ARG-depended NO pathway may benefit more in more aerobic conditions.

D2.S1.1(6). Eccentric hamstring strength: influence on leg stiffness and reactive strength in elite female youth soccer players

\section{DAMIAN HARPER ${ }^{1,2^{*}}$, DALE FORSDYKE ${ }^{1,2} \&$ TOM COMYNS $^{3}$}

\section{${ }^{1}$ York St John University, ${ }^{2}$ York City F.C, F.A Tier 1 Regional Talent Club, ${ }^{3}$ University of Limerick *Corresponding author: d.harper@yorksj.ac.uk @DHMov}

There is strong evidence that eccentric hamstring strength can be increased through high compliance with the Nordic hamstring exercise (NHE), leading to substantial injury rate reduction (of up to $51 \%$ ) in soccer players (Al Attar, Soomro, Sinclair, Pappas, \& Sanders, 2017, Sports Medicine, 47, 907-916). Leg stiffness and reactive strength have important performance and injury implications, however there is a paucity of research that has investigated how eccentric hamstring strength in the NHE may influence these metrics particularly in female and youth populations. Therefore, the aim of this study was to examine the influence of eccentric hamstring strength on measures of leg stiffness and reactive strength in elite female youth soccer players. Following institutional ethical approval eighteen players (age: $14.5+1.1$ years; stature: $1.58+$ $0.06 \mathrm{~m}$; body mass: $49.7+7.6 \mathrm{~kg}$ ) were assessed for: (1) eccentric hamstring strength during the NHE measured using load cells attached to the ankle straps on the Hamstring Solo Elite (NJ Doherty Solutions, Ireland) and (2) leg stiffness (Dalleau, Belli, Viale, Lacour, \& Bourdin, 2004, International Journal of Sports Medicine, 25, 170-176) and reactive strength index (RSI) calculated from the average of the best 5 in a series of 10 maximal repeated bilateral hops (10/5 repeated jump test (RJT), Harper, Hobbs, \& Moore, 2011, BASES student conference). Players were grouped according to average peak eccentric hamstring force $(\mathrm{N})$ of both legs $\left(>228 \mathrm{~N}\right.$ : $\mathrm{ECC}_{\text {highi }}<228 \mathrm{~N}$ :

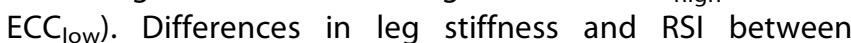
groups were analysed using magnitude-based inferences (Hopkins, 2007, Sportscience, 11, 16-20). Differences in jump height and leg stiffness obtained during the 10/5 RJT were unclear between $\mathrm{ECC}_{\text {low }}$ and $\mathrm{ECC}_{\text {high, whereas }}$ likely lower ground contact times (GCT) and higher RSI were found in players with $\mathrm{ECC}_{\text {high }}(\mathrm{GCT}, 0.19+0.02 \mathrm{~s}$; RSI, $1.34+0.27$ ) compared to $\mathrm{ECC}_{\text {low }}(\mathrm{GCT}, 0.21+0.03 \mathrm{~s}$; $\mathrm{RSI}, 1.14+0.22)$. In addition to reducing injury rates higher eccentric hamstring strength in the NHE can enhance reactive strength qualities mainly by reducing the time on the ground in which jump height is obtained. These findings have important implications for soccer performance in which the production of force during very short ground contact times is repeatedly required. 
D2.S1.1(7). The influence of cold water immersion on markers of recovery following resistance exercise

\section{LAURA WILSON*, LYGERI DIMITRIOU, FRANK HILLS, MARCELA GONDEK \& EMMA COCKBURN}

\section{Middlesex University \\ *Corresponding author: laurawilson1@live.com @Laura_Wilson1}

The use of cold water immersion (CWI) as a recovery intervention is prevalent amongst athletes. Performance of high volume, heavy load resistance exercise is known to result in disturbances of muscle function, perceptual responses and blood borne parameters. Therefore, the aim of this study was to investigate the influence of CWI on markers of recovery following an acute resistance exercise session. With institutional ethics approval, 16 men (mean age: $24 \pm 4.7$ years; stature: $2 \pm 0.05 \mathrm{~m}$; body mass $85 \pm$ $13.5 \mathrm{~kg})$ completed a lower body resistance exercise session (120 repetitions total) comprising back squats, split squats, hip thrusts and Romanian deadlifts, all performed at $80 \%$ predicted $1 \mathrm{RM}$. Participants were match paired into either $10 \mathrm{~min} \mathrm{CWI}\left(10 \pm 0.5^{\circ} \mathrm{C}\right)(n=8)$ or a placebo group $(n=8)$ based on a ratio of predicted 1RM and lean mass. Perceptions of soreness and training stress, markers of muscle function and efflux of intracellular proteins were assessed before, and at 24,48 and $72 \mathrm{~h}$ post exercise. All data were analysed using magnitude based inferences and $90 \%$ confidence intervals. The exercise session resulted in increased muscle soreness, disturbances of muscle function and increases in plasma markers of muscle damage. For perceptions of muscle soreness, there was a possibly trivial effect of CWI at all time points compared to placebo. For isometric peak force there was a possibly harmful effect of CWI at $48 \mathrm{~h}$ post $(-11.5 ; \pm 19.6 \%)$ (mean; $\pm \mathrm{CL}$ ) compared to placebo. For countermovement jump height there was a possibly harmful effect of CWI at $48 \mathrm{~h}(-3.80$; $\pm 10.8 \%)$ and 72 h $(-3.90 ; \pm 10.6 \%)$ post exercise compared to placebo. CWI had a possibly harmful effect on the efflux of creatine kinase $(C K)$ at $24 \mathrm{~h}$ post $(1.53 ; \mathrm{x} / \div 1.881)$ (mean; $\mathrm{x} / \div \mathrm{CL}$ ) and a likely harmful effect at both $48 \mathrm{~h}(1.78 ; \mathrm{x} /$ $\div 1.898)$ and $72 \mathrm{~h}(2.04 ; \mathrm{x} / \div 1.836)$ post compared to placebo. These results suggest that $\mathrm{CWI}$ is not effective at attenuating increases in perceptions of muscle soreness after resistance exercise when compared to a placebo intervention. Further, CWI has a detrimental effect on the CK response, as well as the recovery of isometric peak force and vertical jump performance. The implications of these findings should be carefully considered by athletes and practitioners employing CWI as a recovery strategy following heavy load resistance training. 


\section{Day 2. 5 slides in 5 min Free Communications - Physical Activity for Health}

D2.S1.2(1). The validity and reliability of a smartphone pedometer and the effects of the body characteristics of the user on validity.

\section{JAMES HAZELWOOD*}

\section{The University of Edinburgh \\ *Corresponding author: s1305797@sms.ed.ac.uk}

Smartphone pedometer applications have the potential to provide a wealth of physical activity data or act as a convenient aid in physical activity promotion. However, there are few studies assessing the validity and reliability of these tools and none that take into account the body characteristics of the user. As such, this study aimed to examine the validity and reliability of the smartphone pedometer application "Health" and whether its validity varies with body mass index (BMI) or waist circumference (WC). With ethical approval from the University of Edinburgh, 50 participants (Age $19.76 \pm 1.09$ years, Height $1.74 \pm 0.10$ ) completed two 5 -min walking tests on a treadmill at a self-selected speed $(4.2-6.2 \mathrm{~km} / \mathrm{h})$. Steps were counted by the "Health" application and compared to the criterion measure of manual counting, with validity assessed using paired-samples $t$-tests, $95 \%$ limits of agreement and Bland-Altman plots (Bland \& Altman, 1986, The Lancet, 327(8476), 307-310). The correlation between $\mathrm{BMI}$ or WC and difference between methods was investigated and test re-test reliability assessed using the intra-class correlation coefficient (ICC). On analysis the "Health" application was found to have a mean difference of +69.55 steps $(95 \%$ limits of agreement $-192.85-331.95[P<0.001])$ compared to the criterion measure. Mean differences were smaller and limits of agreement tighter when the pedometer was used by those with a lower BMI or WC. Furthermore, there were significant correlations between error magnitude and $\mathrm{BMI}(r=0.403, P<0.001)$ and WC $(r=0.457, P<0.001)$. "Health" demonstrated "almost perfect" test re-test reliability $(I C C=0.902)$. The results show "Health" to have poor validity which decreases as the user's BMI and WC increases. This precludes its use as a data collecting device and its role in aiding the achievement of an explicit number of daily steps without applying a BMI or WC sensitive correction factor. However, the test re-test reliability is high and so the pedometer could be used to monitor relative changes in activity level. These findings should be considered when appraising studies that measure steps or physical activity using smartphones and further investigation into other fitness wearables is warranted.

D2.S1.2(2). The effects of pool swimming training on maximal oxygen uptake in recreational swimmers or untrained participants: a systematic review and meta-analysis

\section{IAN LAHART* \& GEORGE METSIOS}

\author{
University of Wolverhampton \\ *Corresponding author: i.lahart@wlv.ac.uk \\ @IMLahart
}

Despite swimming's popularity and potential cardiorespiratory fitness enhancing effects, it is has received less scientific attention compared to other exercise modes. Therefore, the aim of this systematic review with meta-analysis was to determine the effect of pool swimming training interventions on cardiorespiratory fitness, assessed via maximal oxygen uptake $\left(V \mathrm{O}_{2} \mathrm{max}\right)$, in recreational swimmers, swimming naïve or untrained participants. To find eligible studies we searched three major electronic databases (PubMed, EMBASE, and CENTRAL) from inception to March 2017, and screened references in relevant reviews and eligible studies. Randomised controlled trials (RCTs), quasi-RCTs, and controlled trials of swimming interventions in non-elite, noncompetitive swimmers, with a measurement of $\mathrm{VO}_{2} \max$, were eligible for inclusion. We did not exclude based on language of publication. Our search of 6,712 records revealed 10 eligible studies. Eight studies included a direct measure of $\mathrm{VO}_{2} \max$, whereas, three studies included an estimated measure. Only one study measured $\mathrm{VO}_{2} \mathrm{max}$ directly during swimming exercise. Swimming had a significant and clinically meaningful effect on relative $\mathrm{VO}_{2}$ max compared to control in an analysis including children/adolescents who were healthy and those with asthma or cystic fibrosis, healthy adults, and participants with hypertension [Mean difference (MD) $6.42 \mathrm{ml} / \mathrm{kg} / \mathrm{min}, 95 \% \mathrm{Cl} 4.34,8.50 ; 1^{2}=60 \%, 8$ studies, 178 participants], and in separate subgroup analyses of children/adolescents with asthma (MD $9.67 \mathrm{ml} /$ $\mathrm{kg} / \mathrm{min}, 95 \% \mathrm{Cl} 5.84,13.51 ; \mathrm{I}^{2}=0 \%, 2$ studies, 32 participants) and healthy adults (MD $5.87 \mathrm{ml} / \mathrm{kg} / \mathrm{min}, 95 \% \mathrm{Cl}$ $2.93,8.81 ; I^{2}=76 \%, 3$ studies, 77 participants). The effect was robust with the inclusion of only RCTs in the combined populations analysis (MD $3.00 \mathrm{ml} / \mathrm{kg} / \mathrm{min}, 95 \% \mathrm{Cl}$ $0.21,5.79 ; 1^{2}=76 \%, 3$ studies, 55 participants). No significant differences in the effects on $\mathrm{VO}_{2}$ max were found between swimming and running in analysis of healthy adults, or in single studies comparing swimming to golfing in children/adolescents with asthma, and cycling in healthy adults. One study found superior effects on $\mathrm{VO}_{2} \max$ with six months of walking versus swimming. However, these findings must be interpreted with caution considering the dearth of RCT evidence, the high/unclear risk of selection, performance, detection, attrition, and reporting bias in eligible studies, and evidence of considerable heterogeneity (i.e., $\mathrm{I}^{2}=50-90 \%$ ) across some comparisons. Swimming may offer beneficial effects on $\mathrm{V}_{2} \max$ across multiple populations and effects may be comparable to other exercise modes. However, these findings are based on limited number of studies. Therefore, RCTs are required to establish the effects of swimming on $\mathrm{VO}_{2} \mathrm{max}$ in healthy populations and those with non-communicable diseases. 
D2.S1.2(3). An exploration of the meaning of physical activity for working adults and their reluctance to reduce sedentary behaviour

\section{KIARA LEWIS ${ }^{2} \&$ NICOLA ECCLES ${ }^{1 *}$}

\section{${ }^{1} C P$ Active, ${ }^{2}$ University of Huddersfield \\ *Corresponding author: nicolaeccles@cpactive.co.uk @CPActiveUK}

The relationship between the working environment and health has been well documented and "choice architecture" and "nudge theory" are gaining traction in public health, yet there is an absence of evidence of how they work and how nudges are perceived by the public (Hollands et al., 2013, $B M C$ Public Health, 13, 1218). This qualitative study aimed to explore the impact of a series of visual prompts to reduce sedentary behaviour and increase activity. With institutional ethics approval subliminal prompts were placed on outdoor media sites, as well as internally in three community work based settings within Kirklees. The initial prompts raised awareness around being sedentary "all day". These were followed by prompts to be more active four weeks later. Invitations were sent to all employees within the work sites and from this 23 participants $(m=6, f=17)$ were recruited and participated twice in focus groups (one pre intervention and one post intervention). The anonymised data, from a total of six focus groups, was analysed using Template Analysis (King and Horrocks, 2012, Interviews in qualitative research. London: Sage). From the initial focus groups 3 main themes were developed. 1. Participants perceive their daily lives as stressful and consider activity as adding to that stress 2 . Being active is seen to require a great deal of effort and willpower 3 . Strong cultural expectations result in people feeling unable to leave their desk. The prompts challenged them to rethink their sedentary behaviour and led to small lifestyle changes and a desire for more help to be active. The data also demonstrated the need to alter the discourse around physical activity in order to appeal to sedentary adults. The research provides a rich source of information on the daily constraints for middle aged, working adults and the difficulties and reluctance they have towards being active. More needs to be done to promote the benefits of an active working day and physical activity as a means to relaxation, rather than another chore at the end of the day.

D2.S1.2(4). The effects of circuit resistance exercise with added upper quadrant training on postural sway and functional fitness in older adults.

\section{SAMUEL OXFORD*, CHRISTOPHER ROSS \& MICHAEL DUNCAN}

\section{Coventry University \\ *Corresponding author: s.oxford@coventry.ac.uk @swoxford}

Falling represents a major public health problem, often leading to progressive functional decline and institutionalisation, (Benichou \& Lord, 2016, Calcified Tissue International, 98(6), 531-545). Maintaining balance involves the integration of visual, vestibular and proprioceptive information while modulating commands to the neuromuscular system (Bisson,
McEwen, Lajoie, \& Bilodeau, 2011, Gait and Posture, 33, 83-87). Core strength training has been shown to be effective for the promotion of balance and functional performance in older people (Granacher, Muehlbauer, \& Gruber, 2012, Journal of Aging Research, 2012, 1-16). Exercise modes that have the potential to promote upper quadrant muscle strength may therefore improve balance performance. To date few studies have examined whether upper quadrant training augments functional changes seen with more traditional training. The aim of this study was to compare the effect of 4 weeks of circuit training exercise vs the same training complimented with upper quadrant motor control exercises on balance and functional fitness in older people. With Institutional ethics approval, sixteen older, but otherwise healthy males $[n=3$; age, $61 \pm 3.4$ years] and females [ $n$ $=13$; age, $63.1 \pm 7.3$ years] participated in 8 weeks of lower and upper body circuit training with the last 4 weeks complimented with upper quadrant motor control exercises performed on MUJO Mechanics equipment. The training intervention was conducted one day a week for 8 weeks. Before, at 4 weeks and post 8 weeks of exercise intervention, postural sway and the best performance from the following functional assessments: Sit to stand, 6 min walk, Arm curl and the 8 foot up and go (Rikli \& Jones, 1999, Journal of Aging and Physical Activity, 7(2) 129-161) were assessed. There were no reductions in mediolateral $(-0.56 ; 95 \% \mathrm{Cl}$ $-3.51,2.39)$ or anteroposterior $(-1.09$; $95 \% \mathrm{Cl}-3.02,0.84)$ $(P>0.05)$ sway from pre-to 4 weeks however, once upper quadrant exercise was added, there were reductions in both mediolateral $(2.38 ; 95 \% \mathrm{Cl} 0.14,4.62)$ and anteroposterior $(1.98 ; 95 \% \mathrm{Cl} 0.34,3.61)$ sway from 4 weeks to 8 weeks (both $P<0.05$ ). There were also improvements in functional assessment $1-4$ and $4-8$ weeks $(P<0.05)$. The present study is the first to demonstrate that upper quadrant motor control movements are effective in alleviating functional consequences of aging, such as balance performance when added to a conventional resistance training programme.

\section{D2.S1.2(5). The application of mental skills} training for improving health in homeless young people: a qualitative case study of MST4Life ${ }^{T M}$

\section{BENJAMIN PARRY ${ }^{1 *}$, SAM COOLEY ${ }^{1}$, MARY QUINTON ${ }^{1}$, MARK HOLLAND ${ }^{2}$, JANICE THOMPSON ${ }^{1} \&$ JENNIFER CUMMING $^{1}$}

\section{${ }^{1}$ University of Birmingham, ${ }^{2}$ Newman University \\ *Corresponding author: bxp092@bham.ac.uk @benjohnparry}

MST4Life ${ }^{\text {TM }}$ is a community-based project in collaboration with a youth housing service (St Basils). Aligned with calls for sport psychology to play an active role in contributing to wider social missions, MST4Life ${ }^{T M}$ applies mental skills training to enhance social inclusion and the physical and mental health of homeless young people. The programme supports homeless young people aged 16 to 25 years in their development of mental techniques (e.g., goal-setting) through psychological (e.g., strengths-based) and pedagogical (e.g., experiential learning) approaches. As part of the programme evaluation, the current study aimed to qualitatively investigate participants' perceptions of health outcomes from 
participating in MST4Life ${ }^{\mathrm{TM}}$. Institutional ethics approval was obtained for this study. Participants in the case study were 16 homeless young people (mean age: $20 \pm 2$ ), living in support accommodation, who completed the MST4Life ${ }^{\text {TM }}$ programme - 10 weekly sessions of experiential, groupbased sessions and a 4-day/ 3-night residential trip to an Outdoor Education Centre. Physical and mental health outcomes were captured through a combination of methods (informal interviews, focus groups and diary rooms) throughout the programme and at 2- to 3-month follow-up. Trustworthiness was established through triangulation of data from three sources: young people, support workers, and outdoor instructors. Data underwent thematic analysis. Themes were generated deductively from theory-based guidelines (e.g., first order themes of physical and mental health) and inductively from data-driven outcomes (e.g., sub-themes serving as evidence form the data). The coding process for data-driven themes was conducted individually by two members of the research team, who then meet to discuss and reach consensus before results were finalised. Results indicate that participants saw an improvement in their perceived physical and mental health. Second order themes of physical health improvements were: re-engaging with physical activity, continued participation in physical activity and better understanding about a healthy lifestyle. Mental health was split into two second order themes of social and psychological outcomes. The key social themes were: tackling isolation and development of social skills (team work and leadership). Psychological outcomes pertained to improvements in perceived self-worth, self-efficacy and resilience. These results suggest the application of sport psychology techniques and approaches can be applied further than a sporting context, to elicit positive changes in physical and mental health of young people with complex and co-occurring problems. Insights from the evaluation of MST4Life ${ }^{\mathrm{TM}}$ can give practitioners a novel approach to working with some of the hardest-to-reach young people in society.

D2.S1.2(6). Accelerometer based physical activity levels, fundamental movement skills and weight status in British preschool children

CLARE ROSCOE $^{1,2^{*}}$, ROB JAMES $^{1}$ \& MICHAEL DUNCAN ${ }^{1}$

${ }^{1}$ Coventry University, ${ }^{2}$ University of Derby

*Corresponding author: roscoec@uni.coventry.ac.uk @clare_roscoe

Preschool children are recommended to participate in a minimum of $180 \mathrm{~min}$ of physical activity (PA) per day for healthy development (Department of Health, 2011, Start Active, Stay Active. London: Department of Health). Objective monitoring of PA via accelerometry provides a useful means to accurately quantify PA behaviour (Phillips, Parfitt, \& Rowlands, 2013, Journal of Science and Medicine in Sport, 16, 124-128). Cut points, have been determined for use with the GENEActiv accelerometers for 3-5 year olds (Roscoe, James, \& Duncan, 2017, European Journal of Pediatrics, 176, 1093-1098). Mastery of Fundamental Movement Skills (FMS) is a prerequisite to functioning on a daily basis (Venetsanou \& Kambas, 2011, Physical Education and Sport, 9, 81-90); they provide the building blocks for future motor skills and PA. Failure to achieve mastery in these skills could prevent preschool children from participating in PA. Therefore, this study aimed to measure PA levels, weight status and FMS in preschool children, to determine whether FMS, weight status and PA influence each other in children of this age. With institutional ethics approval, 185 pre-schoolers (age 4-5 years), from central England wore a triaxial accelerometer (GENEActiv) measuring at 10 s epochs and $100 \mathrm{~Hz}$ for 4 days, including at least one weekend day to determine PA. Height $(\mathrm{m})$ and body mass $(\mathrm{kg})$ were measured, and Body Mass Index calculated. Waist circumference and body mass index were used as a measure of weight status. The proportion of time spent in light, moderate and vigorous PA was determined using specific cut-off points for counts per minute (cpm) related to preschool children. Motor competence was determined using the Test of Gross Motor Development-2. There were no significant differences $(P>0.05)$ between total FMS and PA or total FMS and weight status. This agrees with the Stodden model (Stodden, et al., 2008, Quest, 60, 290-306) as they suggest that the strength of association between FMS and PA and FMS and weight is not strong during the early years. There was also no significant difference $(P>0.05)$ between children that meet the $3 \mathrm{hrs}$ PA guidelines and those that did not. This data is important as it is one of the first to present data on PA levels, FMS and weight status in UK preschoolers.

\section{D2.S1.2(7). Association of active commuting} (walking and cycling) with incident cardiovascular disease, cancer and mortality: findings from the UK Biobank prospective cohort study

\section{ANNE SILLARS, HUI LI, FANNY PETERMANN, NAVEED SATTAR, JILL PELL, STUART GRAY, JASON GILL \& CARLOS CELIS-MORALES*}

\section{University of Glasgow}

*Corresponding author: carlos.celis@glasgow.ac.uk @carloscelism

Active commuting such as walking or cycling has been recommended as a practical way of incorporating more physical activity into daily life; however, there is limited data on mixed-mode commuting and health outcomes. The aim of this study was therefore to investigate the association between active commuting and incident cardiovascular disease (CVD), cancer and all-cause mortality. This study included prospective data from the UK Biobank a population-based study ( $\mathrm{n}=264,337$ participants, $52 \%$ women; mean age 52.6 years). Mode of commuting (walking, cycling, mixed-mode vs non-active (car or public transport)) to and from work was the exposure variable. Outcomes were defined as incident (fatal and non-fatal) CVD and cancer; CVD, cancer and all-cause mortality. The UK Biobank study was approved by the North West Multi-Centre Research Ethics Committee and all participants provided written informed consent to participate in the UK Biobank study. The main results shows that after an average of 5-years follow up cycle commuting and mixed-mode commuting including cycling were associated with lower risk of all- 
cause mortality (Cycle HR: 0.60, [95\% Cl: 0.45-0.81], $P=$ 0.001; Mixed-mode cycle HR: 0.60, [0.46-0.78], $P<0.001$, incident CVD (Cycle HR: 0.64, [0.55-0.74], $P<0.0001$; Mixedmode cycle HR: $0.85,[0.76-0.94], P=0.003)$ and CVD mortality (Cycle HR: 0.43, [0.22-0.81], $P=0.010$; Mixed-mode cycle HR: 0.60, [0.37-0.95], $P<0.0001$ ), incident cancer (Cycle HR: 0.58, [0.50-0.67], $P<0.0001$; Mixed-mode cycle HR: 0.70, [0.63-0.79], $P<0.0001$ ) and cancer mortality (Cycle HR: 0.56, [0.39-0.81], $P=0.002$; Mixed-mode cycle HR: 0.46, [0.32-0.66], $P<0.0001)$. Walking commuting only was not associated with any health outcome. Analyses were adjusted for socio-demographic factors, comorbidities, BMI, smoking, dietary intake, leisure-time physical activity, screen-time and pollution related variables, and conducted as a landmark analysis with follow-up commenced two years after recruitment and including participants who were event-free at this time. In conclusion, cycle or mixed-mode including cycle commuting was associated with lower risk of CVD, cancer and all-cause mortality independent of major confounding factors. Initiatives to encourage and support active commuting could reduce risk of death and the burden of important chronic conditions. 


\section{Day 2. 5 slides in 5 min Free Communications - Psychology (Session 1)}

D2.S1.3(1). Linking coaches' recovery, well-being and athlete performance: a day-level study on antecedents and consequences of autonomy support in elite sport

YANNICK BALK ${ }^{1 *}$, JAN DE JONGE ${ }^{1,2}$, WIDO OERLEMANS $^{1} \&$ SABINE GEURTS ${ }^{3}$

\author{
${ }^{1}$ Eindhoven University of Technology, ${ }^{2}$ University of \\ South Australia, ${ }^{3}$ Radboud University Nijmegen \\ ${ }^{*}$ Corresponding author: y.a.balk@tue.nl \\ @yannickbalk
}

Several studies have found that well-being of coaches is related to their interpersonal behaviour towards athletes (e.g., Stebbings, Taylor, \& Spray, 2016, Journal of Sport and Exercise Psychology, 38, 292-304.). As a lack of recovery has been put forward as an antecedent of high stress levels and poor health of coaches (Lundkvist, Gustafsson, Hjälm, \& Hassmén, 2012, Qualitative Research in Sport, Exercise and Health, 4, 400-419), the aim of the current study was to investigate the role of two recovery experiences, namely physical and emotional detachment, in relation to coach well-being the next morning. Physical detachment refers to shaking off the physical exertion of work, whereas emotional detachment implies that coaches are no longer bothered by negative work-related emotions. Furthermore, the study investigated the consequences of coach well-being in terms of work engagement, autonomysupportive behaviour and athletic performance. The study employed a within-person design over a one-week period. After receiving institutional ethical approval, 31 elite coaches $\left(M_{\text {age }}=38.4 ; S D=7.6 ;\right.$ range $=22-52$ years $)$ and 96 athletes $\left(M_{\text {age }}=21.0 ; S D=3.8 ;\right.$ range $=15-33$ years $)$ completed a daily survey at two time points (after waking up, T1; at bedtime, T2). Indicators of well-being were physical fatigue and positive affect. In addition, we measured coaches' work engagement and athletes' perceptions of autonomy support, engagement and performance satisfaction. Results from multilevel structural equation modelling showed that, for elite coaches, daily physical detachment after work was negatively related to physical fatigue the next morning $(\beta=.14, P=.05)$, whereas emotional detachment was positively related to positive affect the next morning $(\beta=.22, P=.039)$. Both physical fatigue $(\beta=-.17, P=.039)$ and positive affect $(\beta=.16, P=.021)$ in the morning were related to work engagement, which was, in turn, positively related to athletes' perceptions of autonomy support $(\beta=.27, P=.013)$. For elite athletes, daily athlete engagement fully mediated the relation between perceived autonomy support and performance satisfaction $(\beta=.39, P<.001,95 \% \mathrm{Cl}=.27-.50)$. Furthermore, perceived autonomy support positively predicted athletes' performance satisfaction, via athlete engagement $(\beta=.20, P<.001$, $\mathrm{Cl}=.15-.25)$. Taken together, these findings underscore the importance of recovery for elite coaches' well-being. It is highly recommended that coaches consider ways to "switch off" emotionally from work, such as engaging in activities that are different from one's work or spending time with people who do not belong to one's work context. This will benefit their own as well as their athletes' sport experiences.

D2.S1.3(2). Can young athletes differentiate between conditional and unconditional social influence from significant others? An answer from exploratory structural equation modelling

DERWIN KING CHUNG CHAN ${ }^{1,2^{*}}$ \& MARTIN S. HAGGER ${ }^{2,3}$

${ }^{1}$ University of Hong Kong, ${ }^{2}$ Curtin University, ${ }^{3}$ University of Jyväskylä

*Corresponding author: derwin.chan@hku.hk @derwin_chan

Significant others such as coaches, parents, and teammates/ friends play important roles on the sporting experience of young athletes. Indeed, existing research is either interested in certain kinds of social influence based on a theory (e.g., motivational climate from achievement goal theory), or/and investigates only one particular social agent (e.g., parents). There has been a dearth of research that looks at general types of social influence (e.g., positive or negative, conditional or unconditional responses) that could be applicable to different social agents. Theoretically, significant others may exert conditional responses to young athletes according to their performance, for example positive reinforcement (e.g., praise or reward for achievement), and punishment (e.g., punishment for mistakes). On the other hand, they may sometimes also exert unconditional responses such as affiliation (e.g., respect, affection, supportiveness, understanding) and dysfunction (e.g., conflicts, causing negative emotions, lack of respect, negative behaviors) that are unrelated to sport performance. In this study, we use exploratory structural equation modeling (ESEM) to examine young athletes' perception of social influence from significant others. We examined if young athletes could differentiate between 4 hypothesized factors, including two conditional responses (i.e., positive reinforcement, and punishment), and two unconditional responses (i.e., affiliation, and dysfunction). Under this 4-factor structure, sixteen items of Perceived Social Influence in Sport Scale-2 were developed based on the initial version of the scale (Chan, Lonsdale, \& Fung, 2012, Scandinavian Journal of Medicine and Science in Sports, $22,558-568)$, the findings of the qualitative study about significant others in sport (Keegan, Spray, Harwood, \& Lavallee, 2010, Journal of Applied Sport Psychology, 22(1), 87 -105), and screening from two expert panel reviews. After receiving ethical approval from the Institutional Review Board of the first author's university, the items were completed by 904 young athletes from China between 9 to 18 years old (mean age $=14.59, S D=4.13$, range $=6-26 ; 57.5 \%$ male) . ESEM showed that the hypothesized 4-factor-model fitted the data acceptably well, but a 3-factor-model comprising positive influence (Factor 1; positive reinforcement and affiliation combined), punishment (Factor 2), and dysfunction (Factor 3) as 3 independent factors had better psychometric properties 
(Comparative Fit Index $\geq .960$, Tucker-Lewis Index $\geq .935$, and Root-Mean Square Error of Approximation $\leq .088$, $a>.75$, factor loading $>.67$, cross-loading $<.33$ ). In conclusion, young athletes could differentiate between conditional or unconditional responses from significant others ONLY for negative social influence (i.e., punishment and dysfunction), but not for positive social influence (i.e., positive reinforcement and affiliation). It might imply that young athletes were able to identify if feedbacks from significant others were either constructive or intentional, but only exclusively for negative, but not positive, feedbacks.

\section{D2.S1.3(3). Situational antecedents of outward emotional reactions in table tennis}

\section{JULIAN FRITSCH ${ }^{1,2^{*}}$, DIANA ZERDILA ${ }^{3}$, ANNE-MARIE $\mathrm{ELBE}^{2}$ \& ANTONIS HATZIGEORGIADIS}

\section{${ }^{1}$ University of Thessaly, ${ }^{2}$ University of Copenhagen, Denmark, ${ }^{3}$ Democritus University of Thrace *Corresponding author: JulianFritsch@gmx.de}

An emotion is typically defined to involve changes in one's subjective experience, physiological processes, and observable behaviour (Gross, 2015, Psychological Inquiry, 26, 1-26). Looking at the existing literature about the role of emotions in sport, most research has focused on the subjective experience by the use of various self-report questionnaires. At the same time, the observable component in terms of facial expressions, gestures, postures, or verbalizations (i.e., outward emotional reactions) has only received little attention. However, the systematic observation of athletes' outward emotional reactions might be a viable alternative to common practice, thereby, tackling the lack of studies conducted in actual sport competitions. Hence, the main purpose of this study was to look at different situational antecedents of positive and negative outward emotional reactions in table tennis players. Following institutional ethical approval and the consent of participants and coaches, 15 table tennis matches, involving 20 players ( 7 females) with a mean age of 16.70 ( \pm .73 ) were video recorded during the finals of youth National Championship in Greece. Based on the footage, 1921 outward emotional reactions were classified by two coders into three categories: neutral, positive, or negative. Furthermore, each preceding point was classified according to its outcome (won vs. lost), its stage in the tournament (group-phase vs. playoff match), its time point in the match, and its time point in the set. Multinomial logistic regression analysis revealed that point outcome $(P<0.01)$, stage of the tournament $(P<0.01)$, and time point in the match $(P<0.01)$ were significant predictors of positive and negative outward emotional reactions. On the one hand, positive outward emotional reactions were more likely to be observed after winning a point $(B=19.796$; $P<$ $0.01)$ and during play-off matches $(B=-1.316 ; P<0.01)$ and less likely in the first third of a match $(B=-.704 ; P<0.01)$. On the other hand, negative outward emotional reactions are more likely to be observed after losing a point $(B=4.805 ; P<0.01)$, and less likely to be observed in the first $(B=-.641 ; P<0.01)$ and second third $(B=-.377 ; P<0.05)$ of a match. It is intuitive that the outcome of points hugely determines the subsequent outward emotional reactions. The findings that the stage of a tournament and time point in the match are also significant predictors indicate that players should deliberately prepare to deal with the heightened emotional impact in such situations.

\section{D2.S1.3(4). Two case studies of decision-making} under stress

\section{ANNE-CLAIRE MACQUET ${ }^{1 *}$ \& HÉLOÏSE LACOUCHIE ${ }^{2}$ \\ ${ }^{1}$ French Institute of Sports, ${ }^{2}$ Paris Descartes University *Corresponding author: anne-claire.macquet@orange.fr}

High-level athletes operate in dynamic environments and make rapid decisions under stress. This case study aimed to explain how judo players made their decisions in non-routine and challenging events, in order to understand what differentiated them from non-experts. It used the Recognition Primed-Decision model (Klein, 2008, Human Factors, 50, 456-460). According to this model, experts use their experience to make good decisions without comparing alternative courses of action. They assess situations by comparing them with similar previously experienced situations associated with typical actions and stored in memory. If the current situation is analogous to the typical situation in memory, then experts implement the corresponding action and adapt it to the current situation. With institutional ethics approval, two female high-level judokas, ranked within the top 50 judokas worldwide at the time, participated in the study. An interview was conducted with each athlete separately, in which they were asked to describe and comment on the decisions made under stress during an important recent match. Following the Critical Decision Method (Crandall, Klein, \& Hoffman, 2006. Working minds. Cambridge, MA: MIT Press), interviews were used to enable the judokas to describe the decisions made, the decision effectiveness and the processes used to make these decisions. Data processing was carried out using a comparative method (Corbin \& Strauss, 1990, Basics of qualitative research. Newbury Park, CA: Sage). Results showed that under stress the judokas prioritised the use of their favourite technique in two ways: either naturally, in which case the judokas tried to carry out their specific technique directly, or the judokas decided to manipulate a risky situation to make it resemble the typical situation supposed to be stored in memory and associated with the favourite technique, and then implemented the favourite technique. For several minutes, the one-off decision was ineffective at wining the contest; the two-stage decision enabled them to win. Judokas used a situation recognition process to make decisions that was based on four by-products: (a) expectancies (e.g. opponent's tendencies); (b) relevant cues (e.g., opponent's grip); (c) plausible goals (e.g., catch the sleeve); and (c) typical action (favourite technique). Judokas reported differences between themselves (experts) and novices in such situations: ability to maintain concentration despite fatigue, negative emotions and tight score. Results suggest that the ability to turn a risky situation under stress into one the judokas knew how to handle differentiated them from non-experts. Developing situation manipulation under stress could be a useful objective for training.

D2.S1.3(5). The relationship between personality, gender, mental toughness and athletic coping skills in fencers

\section{GERARD BREADEN MADDEN* \& MIKE WESTGATE}

\section{Northern Ireland Fencing Ltd.}

*Corresponding author: gbmadden01@gmail.com @NIFencingLtd 
Fencing is an Olympic combat sport with three disciplines (foil, épée, and sabre), where competitors try to score points ("hits") on their opponent using swords. A highly tactical and mentally demanding sport; a fencer's ability to out-think an opponent and remain focused under pressure is a valuable attribute. Implementing appropriate psychological skills training in fencing may be advantaged by an awareness of certain characteristics of fencers, as well as strengths and weaknesses in their mental skills. With ethical approval from a national coaching institute, ninety-eight UK/Ireland-based fencers (forty-five female) completed questionnaire measures of personality, athletic coping skills, and mental toughness. A distinctive personality profile of fencers emerged; a fencer may be described as outgoing and assertive, self-disciplined, and striving for achievement. Fencers also indicated above-average neurotic traits, suggesting a proneness to experiencing anxiety, self-consciousness, and stress. When evaluated by discipline, sabre fencers demonstrated significantly lower conscientiousness compared to foilists and épéeists, while no gender differences in personality were indicated. Personality traits were used to predict mental toughness and coping skills in hierarchical regression analyses. Neuroticism and Conscientiousness appear useful in predicting coping skills that characterise both fencing discipline and gender, while Extraversion, Openness, and Agreeableness feature less. Two-way ANOVA using fencing discipline and gender as between-subjects factors revealed no significant interaction between these factors for mental toughness or athletic coping skills. However, significant main effects were found for both discipline and gender individually; Foilists indicated significantly less ability to use all aspects of a difficult training environment to their advantage, relative to épéeists and sabreurs $(F(1,96)=$ $1.667 ; P=.037)$. Age may contribute to this effect; foilists were on average the youngest in this sample, and may therefore have less total lifetime training experience. Regarding gender; female fencers demonstrated some characteristics consistent with existing literature, including lower scores in mental toughness $(F(1,96)=6.986 ; P=$ .010), concentration skills (i.e., the ability to focus on the task at hand; $F(1,96)=6.085 ; P=.015)$, and coping with adversity (i.e., remaining positive when things are going badly; $F(1,96)=6.041 ; P=.016)$. Overall, the pattern of results demonstrated is complex, but plausible. Fencing coaches may benefit from an awareness of these patterns in order to prioritise certain types of psychological skills training, or as a means to identifying a specific fencer(s) with a potential skills deficit.

D2.S1.3(6). Psychological resilience, challenge and threat states, and sports performance under pressure

\section{LEE MOORE $^{1 *}$, TOM YOUNG ${ }^{2} \&$ MUSTAFA SARKAR ${ }^{3}$}

${ }^{1}$ University of Bath, ${ }^{2}$ University of South Wales,

${ }^{3}$ Nottingham Trent University

*Corresponding author: I.j.moore@bath.ac.uk @LeeJMoore1

Psychological resilience, or the ability to use personal qualities to withstand stress, is thought to determine who succeeds and fails under pressure (Couto, 2002, Harvard Business Review, 80, 46-55). Moreover, psychological resilience is predicted to aid pressurized sports performance by encouraging athletes to appraise stressful situations as a challenge (Fletcher \& Sarkar, 2012, Psychology of Sport and Exercise, 13, 669-678). However, to date, little research has examined these propositions. Therefore, the aim of this study was to examine if psychological resilience predicted challenge and threat states and performance during a pressurized sporting task. With institutional ethical approval, one hundred participants ( 64 males, 36 females; mean age: $21.94 \pm 4.98$ years) completed the Sport Resilience Scale (SRS; Sarkar, Fletcher, Stride, \& Munir, in preparation, Psychology of Sport and Exercise), before performing a competitive dart-throwing task. Before the task, instructions were given to elevate pressure (e.g., rewards for best performers), and challenge and threat states were assessed via self-report (demand resource evaluation score), and cardiovascular reactivity (challenge/threat index; as Moore, Wilson, Vine, Coussens, \& Freeman, 2013, Journal of Sport and Exercise Psychology, 35, 551-562). After the task, performance was recorded in terms of mean distance from the bullseye (in $\mathrm{cm}$ ). Findings revealed that resilience significantly predicted demand resource evaluations $\left(R^{2}=.07, \beta=.28, P=.005,95 \% C l=0.02\right.$ to 0.09$)$, with greater resilience associated with evaluating the task as a challenge (personal coping resources exceed task demands). Furthermore, resilience significantly predicted challenge/threat index $\left(R^{2}=.03, \beta=.21, P=.043,95 \% \mathrm{Cl}\right.$ $=0.00$ to 0.07 ), with greater resilience related to a cardiovascular pattern consistent with a challenge state (higher cardiac output and lower total peripheral resistance). Resilience also significantly predicted performance $\left(R^{2}=\right.$ $.07, \beta=.27, P=.006,95 \% C l=-0.13$ to -0.04$)$, with greater resilience associated with better dart-throwing accuracy. Finally, mediation analyses revealed significant indirect effects for demand resource evaluations $(95 \% \mathrm{Cl}=0.03$ to 0.19 ), and challenge/threat index (95\% Cl=0.00 to 0.15), implying that resilience benefitted pressurized performance by promoting a challenge state. These findings extend previous research by showing that psychological resilience benefits sports performance under pressure by fostering a challenge state. Practitioners should therefore consider building resilience to aid the performance of athletes who frequently battle with pressure (Fletcher \& Sarkar, 2016, Journal of Sport Psychology in Action, 7, 135-157).

D2.S1.3(7). The relationship between anxiety and depression symptoms and help-seeking intentions in individual sport athletes and university students: the moderating role of gender and participant status

\section{RICHARD TAEHTINEN* \& HAFRUN KRISTJANSDOTTIR}

\section{Reykjavik University \\ *Corresponding author: richard10@ru.is}

Previous studies suggest that athletes may be less likely than non-athletes [Watson, 2005, Journal of College Student Development, 46(4), 442-449] to seek professional help for mental health issues. However, few studies have 
explored the role of current symptoms of common mental disorders in athletes' help-seeking intentions. Hence, this study aimed to explore the relationship between type of symptoms (i.e. no symptoms, anxiety only, depression only, and comorbid anxiety and depression) and helpseeking intentions, and the moderating role of gender and participant status (athlete or non-athlete) in this relationship. The inclusion criteria for all participants was being 18 years or older. The athlete sample consisted of male $(n=85)$ and female $(n=97)$ athletes, competing at the national and/or international level in any of the individual sports that are members of the National Olympic and Sports Association of Iceland. The comparison group consisted of male $(n=101)$ and female $(n=270)$ university students currently enrolled in one of the seven Icelandic Universities. Participants answered an online questionnaire; help-seeking intentions were assessed by the Icelandic version of Beliefs About Psychological Services (I-BAPS); anxiety symptoms by Generalized Anxiety Disorder 7 (GAD-7); and depression symptoms by
Patient Health Questionnaire (PHQ-9). Ethics approval was granted by Icelandic Bioethics Committee. A significant main effect of symptoms on help-seeking intentions was observed among females and this was moderated by participant status $\left[F(3,342)=2.91, P=.035\right.$, partial $\left.\eta^{2}=.03\right]$; female athletes with depression symptoms reported lower intentions than female students with depression symptoms. There was no main effect of symptoms among athletes, but a significant cross-over interaction effect of symptoms and gender on intentions was observed [F (3, 166) $=2.84, P=.040$, partial $\left.\eta^{2}=.05\right]$; non-symptomatic female athletes reported higher intentions than non-symptomatic male athletes, and female athletes with depression symptoms reported lower intentions than male athletes with depression symptoms. Experiencing depression symptoms may decrease female athletes' help-seeking intentions. Hence, although several studies have supported the notion that males have lower help-seeking intentions than females, this relationship may not be as straightforward among athletes as previously indicated. 


\section{Day 2. 5 slides in 5 min Free Communications - Psychology (Session 2)}

\author{
D2.S1.4(1). Examination of factors influencing \\ sport-enjoyment in physical education in \\ adolescents
}

\section{ELIANE STEPHANIE ENGELS* \& PHILIPP ALEXANDER FREUND \\ Leuphana University of Lüneburg \\ *Corresponding author: eliane.engels@leuphana.de}

The importance of sport-enjoyment for a long-term commitment to physical activity is well-established (Scanlan, Carpenter, Schmidt, Simons, \& Keeler, 1993, Journal of Sport \& Exercise Psychology, 15, 1-15). Thus, enjoyment in sport and activity should ideally be promoted in physical education, because in school it is possible to reach a large number of young people. The aim of the present study was to assess the impact of 7 factors influencing enjoyment in physical education. Based on empirical findings (Scarpa \& Nart, 2012, Social Behavior and Personality, 40, 203-204) and backed by social cognitive theory (Pekrun, 2000, Motivational psychology of human development (pp. 143-163)), it was expected, that sportenjoyment depends on factors of social environment (social-relatedness, social handling, autonomy, teachingskills, parental-support) and achievement cognitions (perceived competence, general physical ability). Sport-enjoyment was measured by 3 scales (pleasure: $a=.85$; flowexperience: $a=.65$; recovery: $a=.84$; Authors, submitted). The 7 influencing factors were assessed by newly developed scales ( $a=.78-.85$ ). In a cross-sectional design with institutional ethic approval, $N=1,598$ pupils (female: $50.4 \% ; 12-18$ years, $M=14.39$ years, $S D=1.28$ ) from grades 7 to 10 completed the questionnaire. The results indicated moderate to strong positive and significant correlations of the 3 facets of sport-enjoyment and all 7 influencing factors $(r=.26$ to $.74, P<.001)$. The strongest association was found between flow-experience and social-relatedness and the lowest between autonomy and recovery. Latent regression analysis revealed significant $(P<.05)$ regression coefficients from $\beta=-.12$ to $\beta=.59$. Perceived competence and social relatedness showed the strongest prediction for pleasure. The tested latent structural equation model showed good fit $\left(X^{2}=1324.589, d f=360, P<.001, C F I\right.$ $=.984, \mathrm{TLI}=.980, \mathrm{RMSEA}=.041)$. In conclusion, social relatedness and perceived competence are the most important factors for the experience of sport-enjoyment in school and should be promoted in physical education classes. These findings are in line with results for sportenjoyment in contexts outside school (Scarpa \& Nart, 2012, Social Behavior and Personality, 40, 203-204). The present study features important practical implications for physical education and should facilitate the design of interventions for increasing sport-enjoyment with a view to developing an active lifestyle.

\section{D2.S1.4(2). A case study focusing on the delivery and evaluation of a motivation program for youth sport coaches}

\section{HELEN FERGUSON ${ }^{1 *}$, MICHAEL NOETEL $^{2}$ \& CHRIS LONSDALE $^{2}$}

\author{
${ }^{1}$ University of Wollongong, ${ }^{2}$ Australian Catholic \\ University \\ *Corresponding author: fhelen@uow.edu.au \\ @HelenFerguson10
}

This applied case study focuses on the delivery and qualitative evaluation of a motivation programme for youth sport coaches based on self-determination theory (Ryan \& Deci, 2002, In Deci \& Ryan (Eds.) Handbook of self-determination research (pp. 3-33). Rochester, NY: University of Rochester Press). This programme was delivered to youth sport coaches $(\mathrm{N}=12$; Male $=9$ Female $=3)$ from a range of sports (Soccer, Rugby, Tennis, Netball, Hockey, Ten Pin Bowling, Basketball, \& Cricket). The age of the coaches ranged from 23-74 years old (Mean $=46$ years). The level of the athletes being coached ranged from recreational youth sport athletes to regional academy athletes. The aim of this coach programme was to improve athlete selfdetermined motivation and reduce athlete burnout (adapted from Langan, Blake, Toner, \& Lonsdale, 2015, The Sport Psychologist, 29, 293-301). Fundamentally the programme aimed to provide coaches with ideas of how they could promote autonomy, belongingness and competence for their athletes during their coaching sessions to improve self-determined motivation and enjoyment. The programme is delivered through two face-to-face workshops, 11 online modules and two group mentoring sessions. The online modules include videos depicting good and poor practice to demonstrate how each of the strategies can be used effectively in the coaching environment. Coaches were approached via their sports club and invited to take part in the programme. This case study will provide insights into the lead practitioner's reflections on delivering the programme, as well as feedback from participating coaches, which was obtained through end of session reviews and formal evaluation interviews conducted upon completion of the programme. The challenges associated with the delivery of a comprehensive coach programme to time poor volunteer coaches are explored along with suggestions for overcoming these challenges. The important role of the facilitator is considered to provide suggestions of ways to increase adherence to the programme. In addition, the pivotal role of the first workshop is identified as 'buy in' from coaches at this stage indicated their likelihood of completing the whole programme. Finally, challenges associated with evaluating the effectiveness of the programme are explored along with ways that these challenges were managed. This program is discussed in the context of promoting coaching practice that incorporates 
sport and exercise psychology strategies for encouraging self-determined motivation in youth sport athletes. Recommendations for future practice are provided with suggestions of how the programme could be disseminated to a larger population.

D2.S1.4(3). Methodology goes wild: advocating the advancement of cognitive ethnography in physical activity research

\section{DANNY GOLDING*, GAIL KINMAN \& STEVE KOZUB}

\section{University of Bedfordshire \\ *Corresponding author: danny.golding@beds.ac.uk}

Ethnography has an established pedigree across a range of disciplines and it is somewhat surprising that it is only relatively recently that there has been a proliferation of this research tradition in applied sport and exercise psychology (Krane \& Baird, 2005, Journal of Applied Sport Psychology, 17(2), 87-107). While advocates recognise the unique and rich opportunities such prototypical approaches provide; the potential for exploring cognitive processes has been rather more elusive. However, contemporary developments in other fields of psychology have embraced the ethnographic tradition and have evolved procedures conducive to capturing cognitive processes, once considered elusive to such approaches. This has established a platform for contextual and ecological orientated approaches bringing together two previously perceived incompatible methodologies. Cognitive Ethnography (Ball \& Ormerod, 2000, International Journal Human-Computer Studies, 53(1), 147-168) offers a method of inquiry that prioritises the contextual specificity of cognitive processes by promoting the capture of data in-situ, while developing a realistic and culturally relevant perspective on the phenomenon being investigated. This approach was adopted in two PhD studies which explored stress and coping in an expedition context. Following institutional ethical approval, data was collected as part of expeditions in the Pyrenees and sub-Arctic Norway over periods of 2 and 3 weeks respectively. The research projects used a theoretical framework based upon Transactional models of stress and coping (Lazarus \& Folkman, 1984, Stress, appraisal and coping. New York: Springer) which recognises stress as a cognitive mediational process involving complex and dynamic appraisal processes. Cognitive ethnography provided a suitable field-orientated mechanism for capturing experience and meaning as an alternative to simulated and laboratory investigative techniques typically used within cognitive approaches. It allowed retrospective recall concerns to be addressed and offered a template for recording situated behaviours and cognitive processes. The research projects are the first studies to use cognitive ethnography within an expedition context and have pioneered the use of this approach within sport and exercise psychology. The five slides will briefly outline the distinctive elements of cognitive ethnography using examples from the expedition research projects and focus on what, why and how this methodology can contribute to the field of sport and exercise psychology.

\section{D2.S1.4(4). Mental skills support within elite professional tennis: reflections on a 5-year programme of delivery}

\section{JOHN MATHERS*}

\section{University of Stirling \\ *Corresponding author: j.f.mathers@stir.ac.uk}

Success in professional tennis is associated with the ability to deliver an effective combination of psychomotor skills in elite competitive settings. Despite the lack of empirical evidence to link mental skills (such as imagery, positive self-talk, and goal-setting) to performance outcomes in a professional context, there is substantive belief that such skills can help an athlete succeed in a stressful environment and that these skills can be learned through practice and training (Van Raalte, Cornelius, Hatten, \& Brewer, 2000, Journal of Sport and Exercise Psychology, 22, 345-356). This applied practice case study explores the programme of mental skills support that was delivered to an elite professional male tennis player over a fiveyear period and describes the stages of intervention that were used to frame the service delivery. The participant provided informed consent to allow the delivery approach and content to be shared with the wider world. The intervention stages included: (1) Education/Awareness, (2) Assessment/Profiling, (3) Mental Skills Learning, (4) Application of mental skills in competition, and (5) Evaluation. The mental skills intervention adopted a cognitive-behavioural approach, was embedded within the Individualised Zone of Optimal Functioning Theory (IZOF) and was delivered through a "coaching approach" which was the participant's preferred method of delivery. The programme adopted a "practitioner-led" philosophy in the first year of support, became more "participant-led" in the subsequent years, and culminated with the participant reaching a state of self-sufficiency by the end of the intervention period. The programme of intervention coincided with an improvement in ATP world ranking (Mens Doubles) from number 73 (at the onset of the work) to world number 1 within the fourth year of the intervention period. There were three main delivery dilemmas associated with the work and these were a) the ethics of providing applied support whilst collecting data for the purposes of research, b) managing relationships with the coach and the other member of the doubles team and c) deciding when the applied work should come to an end. These dilemmas were resolved by establishing clear ethical boundaries at the outset of the work, managing client expectations, and working within a clear set of roles and responsibilities whilst attending training sessions and professional events. The reflections of this work are likely to be of interest to applied practitioners and may provide tentative guidelines about how sports psychologists and coaches can work collaboratively to benefit the athlete. 
D2.S1.4(5). The effects of achievement goals and motivational context on psychological functioning and sport performance: an experimental investigation

MAIRI MULVENNA*, JAMES W. ADIE, LUKE SAGE, NIGEL WILSON \& DOUGLAS HOWAT

\section{Coventry University \\ *Corresponding author: mulvennm@uni.coventry.ac.uk @MairiMulvenna}

Integrating tenets of self-determination theory and the hierarchical model of achievement motivation (see Vansteenkiste, Lens, Elliot, Soenens, \& Mouratidis, 2014, Educational Psychologist, 59, 153-174), the current experiment tested the effects of approach-based achievement goal adoption under different motivational contexts on the psychological functioning and performance of competitive participants in a sport setting. A 3 (task-/self-/other-approach goal) $\times 2$ (autonomous/controlling context) repeated measures (time: baseline and post-manipulation performance) design was employed. Following ethical approval, 114 competitive participants $\left(M_{\mathrm{age}}=23.53 ; \mathrm{SD}=4.56\right)$ were asked to perform the first trial (baseline) of a novel basketball shooting task. Participants were then randomly assigned to one of the six experimental conditions and subsequently completed a selfreport measure of stress appraisals prior to performing the shooting task for a second time (post-manipulation). A multisection questionnaire capturing indices of psychological functioning (state anxiety, task enjoyment, perceived competence and goal attainment) was administered at the end of the experiment. A series of 3 (task-/self-/other-approach goal) $x$ 2 (autonomous/controlling context) ANOVAs were conducted on indicators of psychological functioning. A significant twoway interaction $(P<0.05)$ found that the group adopting a task-approach goal under a controlling context appraised the shooting task as significantly more threatening than their counterparts following the same goal under autonomy-supportive conditions. Furthermore, the findings revealed that the task-approach group generally reported higher challenge appraisals, lower cognitive anxiety, higher perceptions of competence and higher goal attainment (all $P<.05$ ) than the other-approach group only. The results also revealed a significant interaction $(P<0.05)$ for the effect of time and context on performance. Specifically, participants following approachbased goals under an autonomy-supportive context significantly improved their shooting performance from the first to the second attempt of the task $(P<0.05)$. Lastly, the findings revealed that those in a self-approach goal condition significantly outperformed their counterparts in the task-approach goal condition, regardless of motivational context $(P<0.05)$. Our findings partially support the notion that the motivational context underpinning the adoption of approach-based goals is salient in determining the psychological functioning and performance among participants in a novel sport situation.
D2.S1.4(6). A neophyte practitioner's reflections on providing sport psychology support to an injured footballer

\section{RACHAEL NEWPORT ${ }^{1}$, DAVID SHEARER ${ }^{2}$ \& CAMILLA $\mathrm{KNIGHT}^{1}$}

\author{
${ }^{1}$ Swansea University, ${ }^{2}$ The University of South Wales \\ *Corresponding author: 921130@swansea.ac.uk \\ @Newport_R
}

Adversity related growth has been identified as an important element for developing elite athletes (Sarkar, Fletcher, \& Brown, 2015, Journal of Science and Medicine in Sport, 18, 475-479). One of these adversity related experiences that an elite athlete can be faced with is a career threatening injury. Salim, Wadey and Diss (2016, Journal of Applied Sport Psychology, 28, 154-169) identified that elite athletes faced with a serious injury, who have a high level of hardiness and a support network to facilitate the management of emotions, are able to reframe their injury into a challenge situation. This current case study explores the reflections of a neophyte practitioner carrying out individual consultancy with a footballer to overcome a career threatening injury and create growth using an Acceptance and Commitment Therapy approach. The individual consultancy was carried out in accordance with the BASES Code of Conduct (2016), BPS Code of Ethics and Conduct (2009) and BPS Practice Guidelines (2017). This consultancy began with a needs analysis, a Performance Profile and Test of Performance Strategies were used to track the changes in the athlete's psychological skills (Thomas, Hardy, \& Murphy, 2007, v3). Seven one-to-one sessions and informal communications were carried out. An ACT approach was chosen, as an appropriate alternative to the commonly used CBT, to encourage rapport building and create change in how the athlete relates to their thoughts. The sessions exposed the challenge of working in a team environment, where it can be difficult to find a confidential, private and psychologically safe space. Furthermore, this case study demonstrated the need for athletes to have a strong social support network in the home and training environment during a serious injury period (Mitchell, Evans, Rees, \& Hardy, 2014, British Journal of Health Psychology, 19, 486-508). Being new to the environment, meant that rapport and trust with the athlete was still in the early stages of development. Conversely, the injury rehabilitation staff had a strong relationship with the athlete and he felt comfortable confiding in them. From re-administration of the Performance Profile and Test of Performance Strategies an improvement in the athlete's emotional control was identified. Therefore, the willingness for athletes to confide in injury rehabilitation staff should be encouraged. It should be welcomed for injury rehabilitation staff to seek education on the value of emotional support during injury periods to maximise and encourage athlete growth. 
D2.S1.4(7). Intrapersonal and interpersonal factors influencing the academic performance of studentathletes at university: a mixed-method case study

\section{OLIVIER ROUQUETTE ${ }^{1,2}$, PHILIPPE GODIN ${ }^{3}$, GUY LORIES ${ }^{3}$ \& ANNE-SOPHIE LEURQUIN ${ }^{3}$}

\author{
${ }^{1}$ Swansea University, ${ }^{2}$ Université Grenoble-Alpes, \\ ${ }^{3}$ Université Catholique de Louvain \\ *Corresponding author:919267@swansea.ac.uk \\ @olivier_rqt
}

Elite athletes studying at university face multiple transitions and challenges at athletic, psychological, psycho-social and academic levels (Stambulova et al., 2015, Psychology of Sport and Exercise, 21, 4-14). Considering regional characteristics of a French speaking university in Belgium, this study aimed to develop a better understanding of interpersonal and intrapersonal factors that influence academic performance of studentathletes. Online questionnaires had been previously developed by the support service at university and were mostly composed of categorical variable and open-ended questions to collect information on the academic-sport combination. With institutional ethics approval, retrospective dataset from 2008-14 (dataset 1, $\mathrm{N}=284$ ) were analysed. Two prospective datasets from January and June 2015 (dataset 2, N = 91; and dataset $3, N=60$ ) included additional questions to assess selfregulatory skills and practices. In addition, six student-athletes (sports science, $\mathrm{n}=2$, human sciences, $\mathrm{n}=2$, engineering and life sciences, $n=2$ ) were interviewed to gain a better understanding of the academic strategies, and specific difficulties encountered due to their dual engagement. Quantitative data were analysed using non-parametric statistics, qualitative data using thematic analysis. Results from dataset 1 showed that from 2008 to $2014,76 \%$ of the student-athlete succeeded at university (pass or above). The dataset 2 highlighted intrapersonal factors associated with academic performance such as having missed classes due to sport participation, $X^{2}(1)=$ 4.484, $P<0.05$; academic self-evaluation, $x^{2}(6)=20.06, P<$ 0.05 ; academic planification, $x^{2}(8)=16.65, P<0.05$; and goal performance setting, $X^{2}(2)=9.29, P<0.05$. The dataset 3 showed that interpersonal factors such as first year university student-athletes were more represented in the lower group of academic performances, $X^{2}(2)=9.49, P<0.05$. The type of study chosen had an impact on academic performance as none of the first-year sports science students were able to succeed in all their exams, and their academic performance was significantly lower than other groups, $X^{2}(3)=9.358, P<$ 0.05 . The qualitative analysis further revealed that fatigue and the cumulative physical load might be a key factor that undermined the academic performance of student-athletes engaged in sports science. In sum, the results suggest that, in addition to intrapersonal characteristics skills and practices, interpersonal factors such as the type of study chosen might have a major influence on the academic performance of student-athletes at university. Further works might consider evaluating the physical and intellectual load combination for student-athletes at university. 


\section{Day 2. 5 slides in 5 min Free Communications - Sport and Performance}

\author{
D2.S1.5(1). Implicit and explicit coordination \\ mechanisms in youth football: temporal \\ adaptations in team cognition and correlations to \\ team performance

\section{MARC BLASER* \& ROLAND SEILER}

\section{University of Bern, Institute of Sport Science ${ }^{*}$ Corresponding author: marc.blaser@ispw.unibe.ch}

Members of a sport team ideally coordinate their actions in a way, that the desired outcome is reached as efficient as possible (Eccles \& Tenenbaum, 2004, Journal of Sport \& Exercise Psychology, 26, 542-560). However, flowing and efficient team action or plays without any coordination errors (e.g. bad pass) are differently coined in sport teams. Particularly in interactive team sports (e.g. football) which are characterised by highly complex, dynamic and uncertain situations, it is an advantage to share a common understanding concerning potential future actions. Otherwise, team members have to communicate (e.g. verbally) their thoughts and ideas what might be impossible due to time pressure and cognitive costs. The aim of the study was twofold: Firstly, we were interested how common understanding (implicit coordination mechanism) and verbal communication (explicit coordination mechanism) develop over time and practice and secondly, how the more appropriate implicit mechanism is related with team performance variables. With institutional ethics approval, 32 under 18 and under 21 youth football players (male, mean age: $17.8 \pm 1.1$ ) performed a football task in teams of two. The task consisted of passing and running elements that are common in football. After a training phase and before two testing phases, players evaluated their actions and the actions of their assigned teammates regarding action type, location, and timing. Out of these evaluations, an accordance index of common understanding was computed. Furthermore, verbal communication during the task was recorded and the whole task itself was videotaped. Team performance variables consisted of time to finish the task, fluidity (e.g. direct passing) and frequency of errors. Results show on one hand that common understanding significantly increased over time and practice $\left(\eta^{2}=.26, P=.003\right)$. On the other hand, verbal communication was reduced, but not on a significant level $\left(\eta^{2}=.13, P=.164\right)$. Furthermore, no significant correlations were found with quantitative team performance variables (e.g. time). Nonetheless, accordance index was significantly correlated with qualitative team performance measures (e.g. fluidity-error-coefficient; $R^{2}=.261, P=.043$ ). The preliminary results indicate a link between implicit and explicit coordination mechanisms: when common understanding increases, verbal communication decreases. Additionally, the correlation between common understanding and team performance is only apparent when qualitative aspects are considered. Further investigation is needed to uncover the oblique relations.

\author{
D2.S1.5(2). Breast support implications for an elite \\ female rower: a multi-disciplinary case study
}

JENNY BURBAGE*, MITCH LOMAX \& CHRIS MILLS

\author{
University of Portsmouth \\ *Corresponding author: jenny.burbage@port.ac.uk \\ @jenny_burbage
}

One elite female rower (22 years, $76.5 \mathrm{~kg}, 1.82 \mathrm{~m}$ ) approached the researchers after suffering with costochronditis, with a concern this was related to their breast support choice. Pain had reduced substantially following a rest period and change in activities (to cycling), yet the athlete sought help identifying appropriate breast support for rowing that would not aggravate this issue or inhibit their performance. The aim of this case study was to investigate whether introducing a high support (HS) bra would affect their biomechanical and physiological responses to rowing. After institutional ethical approval, a 12-min rowing ergometry test (Concept II, USA) consisting of a 3 min warm-up then 3-mins of light-intensity, moderate-intensity and highintensity exercise was completed in the participant's own bra (OB; low compression support) and in a HS bra (36F; Shock Absorber S4490, UK). An iPad (120 Hz; Apple, USA) was used to capture mean trunk flexion (+ve), extension (-ve) and upper arm angles $\left(^{\circ}\right.$ ) at the start of the drive phase, and trunk ROM during the drive phase, in the sagittal plane; video data were analysed using Quintic Biomechanics (UK) software. Expired air was recorded throughout each test using a metabolic cart (Quark CPET, Cosmed, Italy, connected to a small mask). There was little difference $(<$ $2.5^{\circ}$ ) in trunk flexion, extension or total ROM during light and moderate-intensity rowing. Trunk ROM was however $5^{\circ}$ less in the HS bra during high-intensity rowing, attributed to less trunk extension at the end of the drive phase $\left(-39.8^{\circ}\right.$ in the $\mathrm{OB}$ condition compared to $-43.2^{\circ}$ in the HS bra). Upper arm position (mean $3.1^{\circ}$ ) was more vertical in the HS bra compared to the $\mathrm{OB}$ (mean $14.5^{\circ}$ ). The participant tended to pull the rope higher in the $\mathrm{OB}$ condition, changing their arm mechanics. By pulling their arms back a further $\sim 10^{\circ}$ on each stroke, in a lower position, may have benefits for their stroke performance. Ventilation (153 vs. 142.3 I. $\mathrm{min}^{-1}$ ), oxygen uptake (3933 vs. $3882 \mathrm{ml} . \mathrm{min}^{-1}$ ) and carbon dioxide output (4641 vs. $4294 \mathrm{ml} \cdot \mathrm{min}^{-1}$ ) were all lower in the HS compared to the $\mathrm{OB}$ bra in the high-intensity trial. These differences were minimal and could reflect natural unavoidable variation. However, the data do indicate that the HS bra did not negatively affect their physiological performance. The biomechanical results also support the notion that wearing this HS bra does not negatively affect rowing performance, and could actually produce some small positive performance outcomes. 
D2.S1.5(3). Interday reliability and usefulness of reactive strength index derived from the ten to five repeated jump test

\section{THOMAS COMYNS ${ }^{1 *}$, EAMONN FLANAGAN ${ }^{2}$, DAMIAN HARPER $^{3}$, SEAN FLEMING ${ }^{1}$ \& EVAN FITZGERALD ${ }^{1}$ \\ ${ }^{1}$ University of Limerick, ${ }^{2}$ Sport Ireland Institute, ${ }^{3}$ York St John University \\ *Corresponding author: tom.comyns@ul.ie \\ @comyns_tommy}

Reactive strength index (RSI) has been described as an individual's ability to change quickly from an eccentric to a concentric contraction (Young, 1995, New Studies in Athletics, 10, 825-833). RSI has been used in the practical strength and conditioning setting and exercise science literature as a means of quantifying stretch-shortening cycle performance (Flanagan \& Comyns, 2008, Strength and Conditioning Journal, 30, 32-38). A drop jump test has been used extensively to assess an athlete's RSI capabilities. Recently, RSI has been derived from the average of the best 5 jumps obtained from a series of 10 maximal repeated bilateral hops (10/5 repeated jump test (RJT)) (Harper, Hobbs, \& Moore, 2011, BASES student conference). Limited research exists on the interday reliability and usefulness (ability to detect the smallest worthwhile change) of the 10/5 RJT with no reported research for a female population. This study aimed to assess the reliability and usefulness of RSI derived from the 10/5 RJT for an amateur female field sport population. With institutional ethical approval, 15 female participants (mean age: $21.1 \pm 0.9$ years; stature: $1.65 \pm 0.73 \mathrm{~m}$; body mass: $62.0 \pm 5.1 \mathrm{~kg}$ ) (mean $\pm \mathrm{s}$ ) completed 2 trials of the 10/5 RJT with $60 \mathrm{~s}$ rest after a specific warm-up protocol on two testing sessions separated by a minimum of $48 \mathrm{~h}$. A 10/5 RJT technique familiarisation session preceded the two testing sessions. For all $10 / 5$ RJT trials the participants were instructed to minimise ground contact time and maximise jump height. The best trial from each testing day was used for the interday reliability and usefulness analysis. Acceptable reliability was determined at an ICC $\geq 0.8$ and a $\mathrm{CV} \leq 10 \%$ (Hopkins, 2000, Sports Medicine, 30, 1-15). The reported ICC for RSI was $0.91(95 \% \mathrm{Cl} ; 0.76$ to 0.97$)$ and the $\mathrm{CV}$ was $7.3 \%$. In order to assess the usefulness of the test the typical error (TE) was compared to the smallest worthwhile change (SWC). The SWC for RSI in the 10/5 RJT test was 0.09 units (SWC\%: 7.0\%), while the TE was 0.14 units. Therefore the usefulness of this test for this female population is rated as 'marginal' (Hopkins, 2004, Sportscience, 8, 1-7). The results suggest that the 10/5 RJT is a reliable test for RSI as both criteria for acceptable reliability were satisfied but the ability of the test to detect the SWC is marginal.

D2.S1.5(4). A comparison of the hormonal and performance profiles of male Greco-Roman and Freestyle wrestlers during maximal exercise testing

\section{BLAIR CREWTHER*, ZBIGNIEW OBMIŃSKI \& LECH BORKOWSKI}

Institute of Sport, National Research Institute

\section{*Corresponding author: blair.crewther@gmail.com}

Greco-Roman (GR) and Freestyle (FR) wrestling are similar combat sports that employ grappling and throw techniques to pin one's opponent to the mat. However, FR wrestlers can use their legs as weapons and they are permitted to grab their opponents below the waist. These technical differences require specific training methods and adaptations for wrestling success (Obmiński et al., 2016, Journal of Combat Sports and Martial Arts, 7, 29-33). To better characterise these requirements, we compared the hormonal and performance profiles of male GR and FR wrestlers during maximal exercise testing. Under ethical approval, 14 GR (age: $23.3 \pm 3.7$ years) and 14 FR (age: $22.0 \pm 2.7$ years) male wrestlers completed a graded cycling test on a Monark ergometer, starting at 2 $\mathrm{W} \cdot \mathrm{kg}^{-1}$ and increasing by $0.75 \mathrm{~W} \cdot \mathrm{kg}^{-1}$ every $3 \mathrm{~min}$ until exhaustion. Pedalling rate was maintained at $60 \mathrm{rpm}$. Total work, maximal heart rate $(\mathrm{HR})$ and power output were monitored during exercise. Blood cortisol, testosterone and growth hormone $(\mathrm{GH})$ were measured in a fasted state at 8 am ( $3 \mathrm{hrs}$ before testing), before warming up and $3 \mathrm{~min}$ after exercise. The work performed by the GR $\left(2.19 \pm 0.50 \mathrm{~kJ}^{\circ} \mathrm{kg}^{-1}\right)$

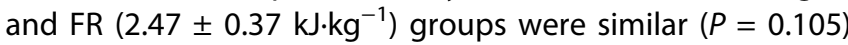
with an effect size (ES) difference of 0.6 , whilst their respective HR $\left(182 \pm 9.7 \mathrm{~b} \cdot \mathrm{min}^{-1} ; 195 \pm 6.5 \mathrm{~b} \cdot \mathrm{min}^{-1}\right)$ and power responses $\left(3.47 \pm 0.38 \mathrm{~W} \cdot \mathrm{kg}^{-1} ; 3.82 \pm 0.37 \mathrm{~W} \cdot \mathrm{kg}^{-1}\right.$ ) favoured the $\mathrm{FR}$ athletes $(\mathrm{ES}=1.5$ and $0.9, P<0.02$ ). Hormone reactivity was similar in both groups. Relative to morning cortisol $\left(606 \pm 130 \mathrm{nmol} \cdot \mathrm{L}^{-1}\right)$ and testosterone $(25.0 \pm 6.28$ $\mathrm{nmol} \cdot \mathrm{L}^{-1}$ ), both hormones decreased significantly prior to warming up ( $E S=-0.9$ and -0.8 ), before subsequently increasing after exercise ( $E S=0.8$ and 2.1). Post-exercise $\mathrm{GH}$ was also elevated $(E S=2.0)$ from warm-up values (3.51 $\pm 4.45 \mathrm{ng} \cdot \mathrm{ml}^{-1}$ ). Stepwise regression revealed that morning cortisol predicted total work $\left(r^{2}=15.4 \%\right)$ and power $\left(r^{2}=\right.$ $15.0 \%)$, whereas the HR response was predicted $\left(r^{2}=16.0 \%\right)$ by training experience $(P<0.05)$. Some biomechanical and physiological differences were identified between male GR and FR wrestlers, but with matching hormonal patterns. For male wrestlers, morning cortisol activity provides a possible individual biomarker of exercise performance several hours later (Obmiński et al., 2016).

\section{D2.S1.5(5). Influence of stochastic movement} patterns on external and internal load measures during simulated rugby league match play

\section{THOMAS MULLEN*, CRAIG TWIST \& JAMIE HIGHTON}

\section{University of Chester}

*Corresponding author: t.mullen@chester.ac.uk @tmullen_sport

Exercise protocols based on time-motion analysis have been devised to replicate the movement and physiological characteristics of rugby league match play (Waldron et al., 2013, International Journal of Sports Physiology and Performance, 8, 484-489), which often comprise repeated cycles of activity. Arguably, these repeated cycles are unlikely to accurately replicate the dynamic movement patterns associated with team sports, given that repetitive actions are associated with 
task-unrelated thinking that can alter the perceptual and physiological response to exercise (Greig et al., 2007, British Journal of Sports Medicine, 41, 908-913). Accordingly, the aim of this study was to assess stochastic order of activity during a rugby league match simulation protocol (RLMSP-i). With institutional ethics approval, eleven male rugby players (age $=21.2$ years, $\mathrm{s}=2$; stature $=1.80 \mathrm{~m}, \mathrm{~s}=0.10$; body mass $=$ $80.5 \mathrm{~kg}, \mathrm{~s}=6.4$; predicted $\mathrm{VO}_{2 \max }=50.8 \mathrm{ml} . \mathrm{kg}^{-1} \cdot \mathrm{min}^{-1}, \mathrm{~s}=$ 3.8) (mean \pm SD) performed two trials of the RLMSP-i, in a randomised order. Trials were matched for movement commands and distances, but the order of events were in a repeated (control, CON) or stochastic (random, RDM) order. Movement distance and speed (via GPS), Stroop test performance (errors and time taken) and blood lactate concentration were measured during all trials, with knee extensor contractile properties (maximum voluntary contraction; MVC, voluntary activation; VA\%) measured pre and post. Subjective task load (NASA-TLX) and session rating of perceived exertion (SRPE) were recorded immediately after the protocol. MVC and VA\% between CON $\left(282.7 \pm 80.7\right.$ N.m ${ }^{-1}$;

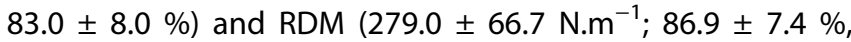
effect size; $E S=0.04$ ) were unclear. Subjective task load was similar between trials (physical demand; $E S=0.30 \pm 0.49$, temporal demand; $\mathrm{ES}=0.29 \pm 0.51$, performance; $\mathrm{ES}=0.46$ \pm 0.79 , effort; $\mathrm{ES}=0.13 \pm 0.57$, frustration; $\mathrm{ES}=0.15 \pm 0.48$ ), with a likely small increased mental demand after RDM (ES = $0.56 \pm 0.57)$. Stroop test time and accuracy were impaired after RDM compared to CON (possibly small; $\mathrm{ES}=0.22$; $\mathrm{ES}=$ 0.30 , respectively). Peak speed across the entire RDM protocol was higher (likely moderate, ES $=0.50$ ), compared to CON, with a likely moderate increase in SRPE after RDM (6.5 \pm 1.3$)$ compared to $\operatorname{CON}(5.5 \pm 1.8 ; \mathrm{ES}=0.52)$. These results suggest that stochastic order of events increases the mental demand, internal and external loads. This has implications for researchers and practitioners using cyclical simulation protocols or training methods that fail to consider the stochastic nature of competitive match play.

D2.S1.5(6). The reliability of integrated external: internal load ratios for field based measures of fitness in rugby union

\section{RICHARD TAYLOR*, DAJO SANDERS, TONY MYERS \& IBRAHIM AKUBAT}

\section{Newman Univesity \\ *Corresponding author: TAYL421@newman.ac.uk @DickiTaylor}

In Rugby Union, weekly competitive matches make regular fitness testing challenging during the in-season period. Previous studies have demonstrated the integrated training load (TL) ratios are valid measures of aerobic fitness (Akubat et al., 2014, International Journal of Sports Physiology and Performances, 9, 457-462), although the reliability and sensitivity of these measures has not been reported. The aim of this study was to assess the reliability and sensitivity of integrated TL ratios in three different training exercises common in rugby union. With institutional ethics approval, twelve academy rugby players from a National League One club competing in the Association of Colleges Elite League agreed to participate in the study (aged; $17.8 \pm 0.5$ years, height; $178.8 \pm 6.4 \mathrm{~cm}$, body mass $81.7 \pm 9.7 \mathrm{~kg}, \mathrm{VO}_{2 \max } 45.8$ $\pm 3.9 \mathrm{~mL} \cdot \mathrm{kg}^{-1} \cdot \mathrm{min}^{-1}$ ). Participants completed an incremental treadmill test for the calculation of the individualised training-impulse (iTRIMP). Players engaged in three field-based training protocols: $760 \mathrm{~m}$ continuous shuttle running (CSR; $20 \mathrm{~m}$ shuttle run at $9 \mathrm{~km} \cdot \mathrm{h}^{-1}$ ), sprint interval training (SIT; $6 \mathrm{x}$ 6 s sprint with 54 s recovery) and a small sided game (SSG; 10 min on a $39 \times 51 \mathrm{~m}$ pitch) which were replicated seven days later. Internal TL was calculated using iTRIMP and external TL measures were collected using a Microelectromechanical systems device (10 Hz GPS, $100 \mathrm{~Hz}$ Accelerometer) included total distance (TD), PlayerLoad (PL), metabolic power (MP), very high-speed distance $>18 \mathrm{~km} \cdot \mathrm{h}^{-1}$ (VHSD), high-speed distance $>15 \mathrm{~km} \cdot \mathrm{h}^{-1}$ (HSD) and individualised high-speed distance based on each player's vOBLA (iHSD). The external TL was divided by the internal TL to form TD:ITRIMP, PL: iTRIMP, MP:iTRIMP, iSHD:iTRIMP, HSD:iTRIMP, VHSD:iTRIMP ratios. Within-subject test-retest absolute reliability was assessed by comparing these ratios for each protocol for the two identical trials. The $\mathrm{CV}$ of the ratios was 7.69 $-16.69 \%$ for the CSR and $7.06-11.49 \%$ for the SIT. CV for TD:ITRIMP, PL:ITRIMP and MP:ITRIMP ratios for SSG 10.5 to $11.7 \%$ respectively. The moderate worthwhile difference (0.6) for the SIT and the SSG protocols were larger than the TEM for all integrated ratios. For the SSG only MP:iTRIMP and HSD:iTRIMP ratios demonstrated a smallest worthwhile difference equal to the TEM. The results of this study suggest that a range of integrated ratios are potentially reliable for monitoring changes in players fitness in rugby union for a variety of training modalities. The TD:iTRIMP, PL:iTRIMP and MP:iTRIMP integrated ratios demonstrated the best reliability during the SIT protocol and MP:ITRIMP during the SSG protocol.

\section{D2.S1.5(7). The effects of $\beta$-alanine on indoor bouldering performance}

\section{DANIEL WOOD*}

Leeds Beckett University

${ }^{*}$ Corresponding author:

d.wood.sportsscience@gmail.com @DWoodSports

Bouldering is a niche discipline of rock climbing devoid of ropes where greater emphasis is placed on the problem solving and gymnastic aspects compared to other climbing disciplines (Philips et al., 2012, Strength and Conditioning Journal, 34(3), 1-18). Exercise induced blood acidosis is an acute physiological condition brought on by extended bouts of high intensity exercise and is regarded as the main cause of falls within climbing literature (Hill et al., 2007, Amino Acids, 32(2), 225-223; La Torre et al., 2009, The Journal of Sports Medicine and Physical Fitness, 49, 19-24). Beta Alanine (B-ALA) has been shown to reduce blood acidosis in high intensity exercise (Hill et al., 2007, Amino Acids, 32(2), 225-223) therefore could have potential ergogenic effect upon climbing performance (Smith, Storey, \& Ranchordas, 2017, International Journal of Sport Nutrition and Exercise Metabolism, 1-28 (in press)). Therefore, the aim of this study was to assess the effects of B-ALA on indoor bouldering performance in an amateur competition setting. 
This is the first study to date to test the effectiveness of a dietary supplement in improving climbing performance. With institutional approval, 10 climbers graded V3-V5 (BALA: $n=5$, Placebo: $n=5$ ) were recruited and assessed with a climbing performance trial (CPT) and in climbing related fitness with an anaerobic fatigue trial (AFT) before and after a 4-week supplementation of $4 \mathrm{~g}$ /day B-ALA or placebo (cornflour). The CPT involved the completion of 10 problems (bouldering climbing routes), measures including overall climbing score, RPE and session RPE (s-RPE). During the AFT participants performed climbing related callisthenic exercises maximally, with performance gauged by the number of reps completed and subsequent fatigue through RPE (Borg, 1982, Medicine \& Science in Sports \& Exercise, 14(5),
674-678). B-ALA improved s-RPE significantly compared to placebo $(P<0.05)$. There were no significant differences found in any other variables measured. This suggests that B-ALA has potential as a training and competition aid within amateur bouldering to suppress perceived effort and therefore improve performance psychosomatically through training. Effects in line with the physiological hypothesis from the findings of (Hill et al., 2007, Amino Acids, 32(2), 225-223; La Torre et al., 2009, The Journal of Sports Medicine and Physical Fitness, 49, 19-24); were recorded anecdotally, which could provide direction for future research in this field. However as these were not measured variables no conclusions can be made at this point, other than B-ALA effects s-RPE. 


\section{Day 2. Posters - Biomechanics and Motor Behaviour}

\section{D2.P1. Does blindfolded balance training work?}

\section{DIANA AVANS*, CHRISTA FRENCH, KEROLLOS MAKRAMALLA \& VERONICA SELWANES}

\section{Vanguard University of Southern California \\ *Corresponding author: davans@vanguard.edu}

Vision plays a significant role in balance. Since nerve fibres from the eyes interact with the vestibular system, researchers have hypothesized that occluding vision in sighted individuals would force the vestibular system to compensate for the loss of visual input resulting in improved balance over time after an initial increase in errors (Nagy, Feher-Kiss, Barnai, et al., 2007, European Journal of Applied Physiology, 100, 97-104; Giagazoglou et al., 2009, European Journal of Applied Physiology, 107(5), 571-579; Proteau, Tremblay, DeJaeger, 1998, Journal of Motor Behavior, 30(2), 143-150). The purpose of this study was to determine if extended blindfolded balance training would encourage this shift to increased proprioceptor use/response and when sight was returned, balance scores would show greater improvement than those that completed the training sighted. The university IRB reviewed and approved the study. Nine males and 15 females, mean age $=21.6 \pm 3.8$ years, completed an 8-week balance training regimen. The Biodex Balance System SD Limits of Stability (LOS) test, a good indicator of dynamic control within a generalized postural sway (2003, Balance Systems Operations and Service Manual, 49), and the Postural Stability Test (PS) evaluating centre of balance, were used pre/post training. The LOS test consisted of three timed trials performed at the recommended stability level of 4 . The PS test was performed at a stability level of 4 to 8 . The participants were paired based on their pre-test scores and then randomly assigned to the experiment or control group. Supervised balance training exercises were performed twice a week, for 30 min per session with the experimental group wearing blindfolds. Every two weeks the exercises were altered and the time held in each was increased to introduce novelty to the proprioceptors and increase strength/endurance. Exercises were chosen based on previous research and recommendations (NHSChoice, 2014, retrieved from http://www.nhs.uk/Livewell/fintess/ Documents/NHS_balance_exercise.pdf; National Institute on Aging, 2015, Exercise and physical activity, http://www.nia. nih.gove/health/publication/exercise-physical-acitivty/sam ple-exercises-balance). Mann-Whitney $U$ analysis found no significant differences between groups in the Limits of Stability test including quadrant measurements $(P=.843)$ or the Postural Stability test $(P=.443)$. Overall, balance showed significant improvement in both groups $(P<0.05)$ but blindfolded training did not significantly increase the improvement. Therefore, our hypothesis was not supported. However, training did improve balance. Many of our participant's balance was poor and they reported feeling improvement in both balance and stability
D2.P2. Controlling the timing of feedback affects
performance gains in a maximum power task LAURA EDWARDS ${ }^{1} \&$ ADAM HAWKEY $^{1,2^{*}}$

\author{
${ }^{1}$ Southampton Solent University, ${ }^{2}$ University of Dundee \\ *Corresponding author: adam.hawkey@solent.ac.uk \\ @a_hawkey
}

Feedback, provided by strength and conditioning (S\&C) coaches, can have a substantial impact on how an athlete performs (Staub et al., 2013, Journal of Strength \& Conditioning Research, 27(8), 2067-2072). Therefore, S\&C coaches need to consider both the timing and frequency of feedback to ensure it is used appropriately. Research has shown that in using feedback, which focuses on the effects of movements (external focus), is advantageous compared to feedback focusing on the movements themselves (internal focus) during the performance of complex motor skills (Jeffreys, 2011, Professional Strength and Conditioning, 20, 30-32). However, it is currently unclear as to the optimum time to provide this feedback with regard to the performance of maximum power tasks. The aim of the current study was to investigate whether the previously observed benefits of an external focus of attention were enhanced when timing of feedback was controlled for tasks requiring maximum power. Following institutional ethics approval one $(n=1)$ male strength athlete (age $=27$ years; mass $=$ $80 \mathrm{~kg}$; height $=1.85 \mathrm{~m}$ ) performed four sets of three Jump Squat (JS) exercises with different conditions of feedback (no feedback, concurrent feedback and terminal feedback) given for each set. A linear transducer (Gym Aware) device, a wire from which was attached to an Olympic barbell with a tethered cord, was used to determine peak bar velocity $\left(\mathrm{m} \cdot \mathrm{s}^{-1}\right)$ and peak power $(\mathrm{W})$ of each jump, both averaged across the jumps. Valid and reliable estimates of force and power $(r=0.86-1.00)$ have been reported during the performance of isoinertial exercise using a single linear transducer system (Cormie et al., 2007, Journal of Strength and Conditioning Research, 21, 424-430) and measurements have been strongly correlated to force platforms $r=0.84$ 0.93 (Crewther et al., 2010, British Journal of Sports Medicine, 44(14)). Results from the current study reported an increase $(13 \%)$ in peak velocity when using feedback compared to no feedback. However, the most crucial finding was external coaching cues given once the athlete had finished the JS (terminal) was $8 \%$ more beneficial in generating peak velocity than providing feedback during the movement (concurrent). These results suggest, for this particular individual, that in order to enhance the production of power and velocity during the JS, S\&C coaches should not only be aware of providing feedback but also when to provide that feedback. Providing feedback after completion of the exercise appears, at least with this athlete, to provide the largest performance gains when peak power is the key training component. 
D2.P3. A positional analysis of the optimal drop jump height of amateur level basketball players

\section{BRADLEY MARSDEN* \& LYSANDER POLLITT}

\author{
Leeds Beckett University \\ *Corresponding author: \\ bradleymarsden@btinternet.com \\ @BradleyMarsden2
}

Lower limb power is vital to basketball performance due to the explosive nature of the sport. To assess dynamic abilities and the effectiveness of plyometric training programmes, drop jumps (DJ) from varying heights are commonly implemented (Byrne et al., 2010). However, considering different physiques and demands across positions, players may not be appropriately stimulated by a general drop height and identifying an optimal drop height $(\mathrm{ODH})$ could be advantageous. While research has acknowledged the potential of an $\mathrm{ODH}$, this has not been established for specific sports or athletes. The main purpose of this study was therefore to identify the $\mathrm{ODH}$ across positions within club-level basketball players. A secondary objective was to determine whether countermovement jump (CMJ) height could be used as an indicator for $\mathrm{ODH} .12$ club-level basketball players, split equally by position (i.e. guards, forwards, centres), completed three familiarisation sessions and one testing session; each involved three maximal CMJ and DJ from $0.3 \mathrm{~m} 0.45 \mathrm{~m}$ and $0.6 \mathrm{~m}$ in a fixed order onto a force platform $(1000 \mathrm{~Hz})$. Reactive strength index (RSI) (i.e. jump height $(\mathrm{JH}) /$ ground contact time) was used to evaluate DJ performance and $\mathrm{JH}$ for CMJ. Natural variability was assessed through a selection of reliability statistics (e.g. coefficient of variation (CV)) during familiarisation. To compare differences between playing positions across drop heights, a mixed ANOVA was used along with effect sizes (ES). RSI was highest at DJ0.3m for guards (129.2) and centres (112.2), however for forwards RSI was similar at DJ0.3m (146.9) and DJ0.45m (147.0). Neither the main effect for RSI at each drop height ( $F=3.476, P=0.053)$, nor the interaction with playing position ( $F=0.364, P=0.831$ ) reached statistical significance. Moderate ES were observed between guards and centres (0.791-0.940) and between forwards and centres (0.820-0.998) at each height. CMJ performance (34.5 \pm 5.3 , range $26.4-43 \mathrm{~cm})$ was not a good predictor for $\mathrm{ODH}\left(\mathrm{r}^{2}=\right.$ 0.443). By the final session, variability reduced across drop heights and was lowest at DJ0.3m (CV 6.6\%), but larger at DJ0.45m (CV 11.2\%) than DJ0.6m (CV 8\%). The results indicate that an $\mathrm{ODH}$ may not exist across playing positions. Although on average the ODH was DJ0.3m, this may not reflect individual responses. Future research must involve larger participant numbers and narrower distinctions between drop heights should be considered. Furthermore, high variability highlights that appropriate familiarisation to the demands is essential during DJ.
Coaches implementing DJ training should consider these findings before exposing players to increasing drop heights.

D2.P4. Case study validation of a linear position transducer for measuring jumping performance

\section{MATTHEW WATSON* \& ADAM HAWKEY}

\section{Southampton Solent University \\ *Corresponding author: 3watsm21@solent.ac.uk @mattwatsonhj}

Athletes, coaches and support staff are taking an increasingly scientific approach to designing and monitoring training programs, Halson 2014, Sports Medicine, 44(2), 139-147). However, with constant technological developments in jump and power based measuring tools, practitioners are seeking reliable equipment that is cost effective and can be used in any training environment Crewther et al., 2010, Sports Medicine, 44(14)). Therefore, the purpose of the current study was to ascertain the validity of a linear position transducer (LPT: GymAware Power Tool, Kinetic Performance Technologies, Canberra, Australia) when compared against a gold standard portable force platform (PFP: Kistler Quattro Jump 9290CD) for the measurement of peak force (PF) and peak power (PP) during a jump squat (JS). Following institutional ethics approval one $(n=1)$ male participant (age $=26$ years.; height $=1.92 \mathrm{~m}$; mass $=79.5$ $\mathrm{kg}$ ), with international track and field experience, $>$ six years weight training history, and proficiency in JS, volunteered for the current study. The participant completed four sets each of three single JS repetitions at $20 \mathrm{~kg}, 30 \mathrm{~kg}, 35 \mathrm{~kg}$ and $40 \mathrm{~kg}$ respectively. The JS was used due to the eccentric-to-concentric contraction having a strong correlation within sporting power based movements Cormie et al., 2007, The Journal of Strength \& Conditioning Research, 21, 424-430). To ensure parity of results, PP and PF were measured from the JS on both testing apparatus simultaneously. The JS depth was self-selected, ensuring a fast eccentric to concentric contraction to utilise elastic energy storage, in accordance with Bobbert et al., 1996, Medicine \& Science in Sports \& Exercise, 28(11), 1402-1412. Results showed $\mathrm{PF}$ and PP both had a strong positive linear relationship for the LPT and PFP ( $r=0.87$ and $r=0.83 \& r=0.87$ and $r=0.80)$ and the correlation between the testing methods for both parameters suggested strong significance $(P=0.06 ; P=0.08)$. Results from the current study suggest that the LPT shows strong correlation to the gold standard PFP when used to measure JS in a training environment. These findings, combined with the reported simplicity, portability, and relatively low cost of using the LPT Harris et al. (2010, The Journal of Strength \& Conditioning Research, 32(4), 66-79), provide strong evidence for its incorporation into a sports coaching setting. Further investigation is now required to ascertain the LPT's effectiveness in measuring other power based exercises. 


\section{Day 2. Posters - Physical Activity for Health}

D2.P5. Construct validity of the resistance training skills battery in children aged 7-10 years

\section{MICHAEL DUNCAN*, CHELSEY LAWSON, RUTH BOAT, JASON TALLIS, LEANNE RAYMOND, VICTORIA JONES \& EMMA EYRE}

\author{
Coventry University \\ *Corresponding author: \\ michael.duncan@coventry.ac.uk \\ @mikedunky
}

The Resistance Training Skills Battery for Children (RTSBC) has recently been developed as a measure of resistance training skill competence and readiness to undertake loaded resistance training in children and youth (Lubans et al., 2014, Journal of Strength and Conditioning Research, $28,1373-1380)$. RTSBc scores were significantly related to field based tests of muscular fitness in adolescents aged 12-16 years, evidencing construct validity of this measure (Lubans et al., 2014). No studies to date have examined the validity of the RTSBC in children younger than 12 years of age. The present study examined this issue. Following institutional ethics approval and informed parental consent, 27 children (21 males, 6 females) aged 7-10 years (Mean age \pm $\mathrm{SD}=8.3 \pm 1.3$ years) took part in this study. The RTSBC was determined using video analysis of six skills (suspended row, push-up, step-up, body-weight-squat, front support and overhead-press) following the guidelines of BebichPhilip et al. (2015, Pediatric Exercise Science, 28, 473-480). General Motor Competence was assessed using the Test of Gross Motor Development-2 (TGMD2, Ulrich, 2001, Test of Gross Motor Development, Texas: PRO-ED). Muscular fitness was assessed using a seated $1 \mathrm{~kg}$ medicine ball throw (MBT) and standing long jump (SLJ). $10 \mathrm{~m}$ sprint speed was also assessed using infrared timing gates (Fusion Sport, Coopers Plains, Australia). Pearson's product moment correlations indicated that RTSBC scores were significantly related to TGMD2 scores $(r=.875, P=0.001)$, MBT $(r=.400$, $P=0.047), \mathrm{SLJ}(r=.511, P=0.001)$ and sprint speed $(r=$ $-.722, P=0.001)$. Using a median split for RTSBC scores, children were categorised as high or low resistance training competence. Univariate ANOVAs were then used to examine whether measures of muscular fitness and TGMD2 scores differed as a function of RTSBC competence. Results indicated that children who were classified as high for resistance training competence had significantly higher TGMD2 $(P=0.001)$, MBT $(P=0.047)$ and SLJ $(P=0.034)$ scores and significantly faster $10 \mathrm{~m}$ sprint speed $(P=0.001)$. This study demonstrates construct validity of the RTSBC as measure of general motor competence and muscular fitness in children aged 7-10 years. The current study also supports assertions (Lubans, et al., 2014), based on adolescent data, that the RTSBc can be used to determine competency and readiness for resistance training in pediatric populations.
D2.P6. Beneficial effects of a modest enhancement of physical activity in pain perception: a preliminary study using quantitative sensory testing and electroencephalography

\section{ANNE KING*, PHOEBE BLACKNEY, STUART EGGINTON, CATHERINE GRAHAM, RAE LARMOUR, ELEANOR NUNNS \& SAMANTHA WAITE}

\author{
University of Leeds \\ ${ }^{*}$ Corresponding author: a.e.king@leeds.ac.uk
}

Exercise is a recommended non-medical intervention for chronic pain (Rasu, Sohraby, Cunningham, \& Knell, 2013, Journal of Pain, 14, 568-578). However more data is needed to inform an evidence-based approach to the concept of "exercise as a prescription for pain"'. In this preliminary study, we utilised quantitative sensory testing (QST) and recordings of contact heat-evoked potentials (CHEPS) within the electroencephalograph (EEG) to assess the impact of a short-term exercise programme on pain perception in healthy participants. All protocols had local ethical approval. Participants ( $n=22 ; 11$ females, 11 males, age $23+7$, normal BMI range) undertook a short-term exercise programme (duration 1-2 weeks; target of $25 \%$ increase daily steps relative to a control baseline). Established QST protocols were used for measurement of cutaneous hot pain threshold (HPT), cold pain threshold (CPT) and pressure pain threshold (PPT). CHEPS were recorded using an EEG methodology (BrainVision, UK) with thermal stimuli $\left(49-51^{\circ} \mathrm{C}\right)$ applied to the forearm surface (Pathway System, MEDOC, Israel). During the EEG study, participants used a numerical rating scale (NRS, 1-10) to verbally report their subjective pain intensity. Data are means \pm S.E.M. and unpaired Student's t-tests were used for statistical analyses with significance set at $P<0.05$. A Cohen's $d$ value was calculated to infer a small $(<0.02)$, medium (0.2-0.5) or large ( $>0.5)$ effect size. Post-exercise, there was a significant increase in HPT temperature (from $44.73 \pm 0.70$ to $46.89 \pm 0.49{ }^{\circ} \mathrm{C}, P<0.05 ; d=0.85$ ) whilst the CPT temperature was reduced (from $10.86 \pm 2.19$ to $4.98 \pm$ $6.42{ }^{\circ} \mathrm{C}, P<0.05 ; d=0.75$ ) indicating hypoalgesia and increased resilience to either hot or cold pain. The PPT showed a non-significant trend to increase from the control value of $489.83 \pm 56.34$ kilopascal $(\mathrm{kPa})$ to a post-training value of $615.48 \pm 56.44 \mathrm{kPa}(P>0.05 ; d=0.52)$ indicative of hypoalgesia. Within the EEG, the CHEPS amplitude posttraining $(16.70 \pm 1.85 \mu \mathrm{V})$ was significantly lower compared to pre-exercise values $(19.94 \pm 2.47 \mu \mathrm{V} ; P<0.05 ; d=0.53 ; \mathrm{n}=$ 4) with a concomitant reduction in verbally reported pain rating NRS values (control of $7.13 \pm 0.48$ compared to $5.13 \pm$ 0.83 post-exercise; $P<0.05 ; d=0.88$ ). Overall, these preliminary data suggest that modest, short-duration physical activity can positively influence an individual's pain experience. Extended studies are required to validate these data and to determine if such programmes may be beneficial to those with chronic pain. 
D2.P7. Validity of the novel Isometric Exercise Scale (IES) for measuring rating of perceived exertion during isometric exercise training

\section{JOHN W. D. LEA*, JAMIE M. O'DRISCOLL \& JONATHAN D. WILES}

\section{Canterbury Christ Church University \\ *Corresponding author: John.lea1@canterbury.ac.uk @sportscipodcast}

Isometric exercise (IE) training interventions have been shown to be effective at reducing resting arterial blood pressure (O'Driscoll et al., 2017, Physiological Reports, 5, e13236). IE intensity is generally determined using torque electromyography (EMG), heart rate (HR), or knee joint angle; however, rating of perceived exertion (RPE) has been shown to be a valid and useful measure of IE intensity (Hummel et al., 2005, European Journal of Applied Physiology, 95, 321-326) and may provide a more accessible means of determining IE workload. The RPE scales currently being used were not specifically designed for use with IE; therefore, the aim of this study was to assess the validity of the novel "Isometric Exercise Scale" (IES) during a continuous incremental isometric wall squat test. Following institutional ethical approval, 29 male participants (age: $23.2 \pm 4.0$ years; stature: $180.9 \pm$ $7.8 \mathrm{~cm}$; body mass: $82.7 \pm 17.3 \mathrm{~kg}$; BMl: $25.2 \pm 4.5 \mathrm{~kg} \cdot \mathrm{m}^{-2}$ ) (mean $\pm \mathrm{s}$ ) completed 4 isometric tests, on separate occasions, with $48 \mathrm{~h}$ in-between. Each test consisted of 5 isometric wall squat intensities, determined by knee joint angle from $135^{\circ}$ to $95^{\circ}$ (easiest to hardest respectively) in $10^{\circ}$ increments; each intensity was held for 2 min (Goldring et al., 2014, Journal of Sport Sciences, 32, 129-136). The test was continuous, with no breaks between intensities, and the test continued until volitional fatigue or completion of the 5 work stages. Throughout the exercise protocol, RPE (IES and Borg's CR-10), HR and blood pressure (BP) were recorded. Construct validity was established by correlating RPE from the CR-10 scale and the IES, using linear regression analysis. Concurrent validity of the IES scale was measured by correlating the IES results with: Time-under-tension, HR and BP. Construct validity results showed a strong positive and linear relationship $(r=0.967, P<0.001)$ between the CR-10 and IES results. Concurrent validity coefficients showed positive linear relationships between the IES and Time-under-tension $(r=0.849, P<0.001), \mathrm{HR}(r=0.819, P<$ $0.001)$ and $B P(r=0.841, P<0.001)$. These results suggest that the IES is a valid measure of IE intensity; additionally, the IES showed good agreement with the Borg CR-10 scale. As such, the IES can be used as a useful measure of exercise intensity during IE.

D2.P8. Health regulatory foci promotion and prevention, selection optimization and compensation strategy and physical activity: a meditational analysis

\section{MANON LAROCHE*, PEGGY ROUSSEL \& FRANÇOIS CURY}

\section{Aix Marseille University \\ *Corresponding author: manon.laroche@univ-amu.fr}

To date, several studies have demonstrated the relevance of Regulatory Focus Theory (Higgins, 1997, American
Psychologist, 52, 1280) in the health research area (Gomez, Borges, \& Pechmann, 2013, Journal of Consumer Psychology, $23,451-463)$. In a health-related context, a regulatory focus promotion is reflected by a concern for health-related gains and opportunities, whereas a regulatory focus prevention is reflected by a concern for health-related problems and mistakes. In this study, our purpose was to examine the impact of these health regulatory foci on physical activity, and to evaluate in what extent this impact could be explained by the use of Selection, Optimization and Compensation (SOC) strategy (Freund \& Baltes, 2002, Journal of Personality and Social Psychology, 82, 642). A cross-sectional study (web survey) was conducted with 513 volunteer participants ( 265 men) aged from 18 to 82 years $\left(M_{a g e}=36.95, S D=16.33\right)$. With institutional ethics approval, the participants completed self-report questionnaires measuring health regulatory foci promotion and prevention (Gomez et al., 2013), health condition (Kempen, Jelicic, \& Ormel, 1997, Health Psychology, 16, 539-546), Physical Activity (PA) (Robert et al., 2004, Annales de Réadaptation et de Médecine Physique, 47, 546-554), and SOC strategy used in physical activity (Ziegelmann \& Lippke, 2007, International Journal of Behavioral Medicine, 14, 30-39). First, simultaneous multiple regressions were conducted on PA and on SOC strategy. The basic regression model was composed of health promotion focus, health prevention focus, gender, education level, age, and health condition. When regressing PA $\left(R^{2}=\right.$ $0.17)$, the main results showed that health promotion focus $(\beta=0.20)$ was a positive predictor whereas health prevention focus $(\beta=-0.11)$ was a negative predictor. When regressing SOC strategy $\left(R^{2}=0.22\right)$, health promotion focus $(\beta=0.41$ ) was found to be a positive predictor. Secondly, SOC strategy was included in the basic model $\left(R^{2}=0.24\right)$ and was found to be a positive predictor of PA $(\beta=0.30)$. Finally, Bootstrap procedure revealed that SOC strategy partially mediated the positive relationship between health promotion focus and PA. For the first time in the literature these results suggest that health regulatory foci are related to $\mathrm{PA}$, and that the positive relationship between health promotion focus and PA is in part explained by the use of SOC strategy. Thus, health promotion focus (not health prevention focus) seems to generate in individuals a capacity to manage themselves proving conducive to physical activity.

D2.P9. Test-retest agreement of a novel portable sit-to-stand device in adults aged $20-70$ years

\section{PETER MUNDY, JASON TALLIS, SAM OXFORD, EMMA EYRE \& MICHAEL DUNCAN}

\section{Coventry University \\ *Corresponding author: ab9674@coventry.ac.uk @Peter_Mundy}

The sit-to-stand (STS) test is a well-used and validated test of lower limb muscle function (Tsuji et al., 2015, International Journal of Gerontology, 9, 111-118). Commonly, practitioners assess the time taken to complete a set number of STS repetitions or total number of repetitions performed in 30-s (McCarthy et al., 2004, Journal of Gerontology A, 59, 
1207-1212). However, such tests do not always reflect lower limb function because they are influenced by muscular endurance. The Tanita Zaritz is portable device which has been developed to assess muscle strength and speed during STS but, to date, no data on its test-retest agreement are available. Following institutional ethical approval and informed consent, 105 20-70 year old adults (57 males, 48 females) performed two sets of STS trials on the Tanita Zaritz (Tanita Inc, Tokyo, Japan). Test-retest agreement for measures of leg muscle strength (Peak Force) and leg muscle speed (Rate of Force Development) were determined using 95\% Limits of Agreement (LoA) (Atkinson \& Nevill, 1998, Sports Medicine, 26, 217-38). Results indicated acceptable agreement for leg muscle strength $(95 \%$ LoA $=0.13)$ with low levels of systematic bias (Bias $=0.00$ ). Further, the results were similar for leg muscle speed, with acceptable agreement $(95 \%$ LoA $=2.19)$ and low levels of systematic bias (Bias $=0.07$ ). This study indicates that test measurements taken using the Tanita Zaritz device demonstrate acceptable levels of agreement, with a low level of bias. Therefore, the Tanita Zaritz is reliable, time-efficient, and portable means to assess lower limb muscle function in adults aged 20-70 years.

\section{D2.P10. An aerobic exercise intervention in} overweight women decreased fat mass but was partially compensated by increased appetite but not by increased sedentary behaviour or decreased non-exercise physical activity

\section{ANNA MYERS*, MICHELLE DALTON, CATHERINE GIBBONS, GRAHAM FINLAYSON \& JOHN BLUNDELL}

\section{Sheffield Hallam University \\ *Corresponding author: a.myers@shu.ac.uk @anna_myers1}

Exercise-induced weight loss is often less than expected and highly variable between individuals. This implies some degree of compensation in response to the exercise-induced energy deficit. Given that energy intake (EI) and non-exercise physical activity (NEPA) and sedentary behaviour (SB), are major determinants of body weight, compensation in either of these could undermine the exercise-induced energy deficit and compromise weight loss. The aim of this study was to examine changes in body composition, appetite, NEPA and SB in response to a 12-week supervised and monitored aerobic exercise intervention in overweight and obese women. Twenty-four women aged 33.1 years $(S D=11.7)$ with a body mass index (BMI) of $27.9 \mathrm{~kg} / \mathrm{m}^{2}(\mathrm{SD}=2.7)$ completed 12-weeks of supervised exercise $(500 \mathrm{kcal}, 5$ times per week). Body weight, waist circumference (WC), body composition, resting metabolic rate (RMR), total daily $\mathrm{El}$ and subjective appetite sensations were measured at baseline (weeks 0 ) and week 12. Free-living PA and SB were measure at baseline, week 1 and 10 of the exercise intervention, and at post-intervention. NHS ethical approval was obtained prior to any measures being taken. There was a significant reduction in body mass $(M=0.83 \mathrm{~kg}, \mathrm{SD}=1.85)$, BMI ( $\left.M=0.30 \mathrm{~kg} / \mathrm{m}^{2}, \mathrm{SD}=0.66\right), \mathrm{WC}(\mathrm{M}=3.62 \mathrm{~cm}, \mathrm{SD}=$ $3.85)$, fat mass ( $F M ; M=1.50 \mathrm{~kg}, S D=2.18)$ and a significant increase in fat-free mass (FFM; $M=0.67 \mathrm{~kg}, \mathrm{SD}=0.98$ ). There was an increase in total $[P=.028]$, ad libitum $[P=.030]$ and snack box El $[P=.048]$. This was accompanied by an increase in hunger $[P=.016]$ and a decrease in fullness $[P=.040]$ throughout the day during week 12 of the exercise intervention compared with baseline. There was no evidence for a compensatory reduction in NEPA $[P=.99]$ and no increase in $\mathrm{SB}$ as a result of increased structured exercise $[P=.03]$. In fact exercise displaced SB. Twelve weeks of supervised aerobic exercise resulted in significant weight (FM) loss and an increase in FFM. There was a compensatory increase in El in response to the exercise that was associated with an increase in hunger and a decrease in fullness throughout the day. There was no evidence for a compensatory reduction in NEPA or an increase in SB. The structured exercise seemed to displace some SB.

\section{D2.P11. The acute physiological effects of high} and low velocity resistance exercise in older adults

\section{DARREN RICHARDSON ${ }^{1 *}$, MICHAEL DUNCAN ${ }^{1}$, ALFONSO $^{*}$ JIMENEZ ${ }^{1}$, VICTORIA JONES ${ }^{1}$, PAUL JURIS ${ }^{2}$ \& NEIL CLARKE $^{1}$ \\ ${ }^{1}$ Coventry University, ${ }^{2}$ UMASS Amherst \\ *Corresponding author: richa190@uni.coventry.ac.uk}

Resistance exercise has been explored as a means of improving functional performance in older adults, with much debate on whether high (HVE) or low (LVE) velocity resistance exercise is more effective (Tschopp, Sattelmayer, \& Hilfiker, 2011, Age Ageing, 40, 549-556). However, investigations into these two modes of resistance exercise in older adults have largely failed to examine acute physiological changes. The aim of the present study was to determine if workload matched, high velocity (HVE) and low velocity (LVE) resistance exercise elicit differing acute physiological responses in older adults. Following institutional ethics approval, ten recreationally active older adults (Five males: age: $66 \pm 3$ years, and five females: age: $68 \pm 2$ years), completed three sets of bicep curl, leg press, calf raise, leg extension, leg curl, seated row, chest press and tricep extension on six separate occasions (Three HVE and three LVE sessions). Systolic blood pressure, diastolic blood pressure and blood lactate were measured at rest and immediately post-exercise. Heart rate and rating of perceived exertion were measured post warm-up, and after each set of every exercise. There appeared to be no significant differences in lactate responses between HVE $\left(4.3 \pm 2.1 \mathrm{mmol} \cdot \mathrm{L}^{-1}\right)$ and LVE $\left(4.6 \pm 2.8 \mathrm{mmol} \cdot \mathrm{L}^{-1}\right)(\mathrm{F}(1,9)=0.028 ; P=0.872 ; 95 \% \mathrm{Cl}$ : $-0.6,0.7 ;=0.003)$ and heart rate responses were not significantly different between HVE and LVE $(F(1,9)=0.045 ; P=$ 0.837; 95\%Cl: $-3.6,3.0 ;=0.01)$. Furthermore, systolic blood pressure responses were similar following HVE (139 \pm $19 \mathrm{mmHg})$ and LVE $(136 \pm 18 \mathrm{mmHg})(\mathrm{F}(1,9)=0.023 ; P=$ $0.884 ; 95 \% \mathrm{Cl}:-5.3,6.0 ;=0.003)$ and diastolic blood pressure responses were also similar for HVE $(72 \pm 7 \mathrm{mmHg})$ and LVE $(74 \pm 9 \mathrm{mmHg})(\mathrm{F}(1,9)=1.516 ; P=0.249 ; 95 \% \mathrm{Cl}:-3.8,1.2 ;=$ 0.14). However, ratings of perceived exertion were significantly greater following LVE $(14 \pm 0)$ compared with HVE (12 $\pm 1)(\mathrm{F}(1,9)=13.059 ; P=0.006 ; 95 \% \mathrm{Cl}: 0.4,1.8 ;=0.59)$. Clear recommendations cannot be drawn from the findings of the present study, nevertheless HVE may be a more appealing 
mode of resistance exercise to prescribe to older adults, as it may produce a comparable physiological stimulus to LVE while being perceived as significantly less exerting. Exercise practitioners and those working with older adults might therefore want to employ HVE preferentially given the link between increased exercise intensity and reductions in pleasure (Ekkekakis, Parfitt, \& Petruzzello, 2011, Sports Medicine, 41, 641-671). Consequently, further investigation of the affective responses to both HVE and LVE would be a beneficial next step in clarifying the general recommendations for older adults.

\section{D2.P12. Biomechanical correlation analysis of} capoeira movement "meia lua de compasso", among three different-level practitioners in Bogota

MANUEL A. RIVEROS, JOSE L. GARCIA, CARLOS E. MEL, ADRIANA R. GUTIEREZ, CARLOS A. COLLAZOS, OSCAR F. RUBIANO*, ANGELA MARIA RODRIGUEZ \& JEISSON ESTEBAN MONTES

\section{Manuela Beltrán University \\ *Corresponding author: oscar.rubiano@umb.edu.co}

Capoeira is a martial art originated from Afro-Brazilian culture. In Brazil, Capoeira is considered the national sport. Capoeira practice has expanded internationally due to the migration of masters practicing this martial art. There are few scientific studies on capoeira biomechanics. The objective of this study was to correlate kinetic and kinematic variables during vertical jump tests among three different level of capoeira practitioners during "meia lua de compasso" movement. The study was approved by the Manuela Beltrán university ethics committee. The vertical jump tests were measured using a BTS 6000 force platform according to protocols established to obtain Maximum-Strength Peak (MEP) data measured in Newtons (Nw) and, Maximum Speed Displacement (MSD) in meters per second $(\mathrm{m} / \mathrm{s})$ with both, right and left limb. Participants were 18 subjects from both genders (men $=9$ ), classified into beginner (BEG), intermediate (INT) and advanced (ADV) level. For each level, there were 3 men with $X^{-}$age of $27 \pm 6.2$ years and 3 women with $(X)^{-} 25 \pm 5.1$. Other measurements included body composition using an Omron HBF-516B impedance meter scale. The mean results for MSD by gender at the beginner's level for males were $11 \mathrm{~m} / \mathrm{s}$ for the right and left side. Females' MSD mean was $7.13 \mathrm{~m} / \mathrm{s}$. Intermediatelevel male participants MSD was $13.50 \mathrm{~m} / \mathrm{s}$ and female mean $11.5 \mathrm{~m} / \mathrm{s}$. Males' advanced level mean values was $14.88 \mathrm{~m} / \mathrm{s}$ and females' mean value was around $12.2 \mathrm{~m} / \mathrm{s}$. Values for MEP were: $702.23 \pm 55.9 \mathrm{Nw}$ for male, female $611.80 \pm 200.2$ $\mathrm{Nw}$ at the beginners' level; $715.50 \pm 155.7 \mathrm{Nw}$ male, female $526.25 \pm 49.4 \mathrm{Nw}$ at the intermediate level and, 756.51 level $\pm 167.7 \mathrm{Nw}$ in male, $528.79 \pm 20.8$ for female participants at advanced level. Results indicated non-statistical correlation for training land MEP (Spearman's Rho $r=-0.14 ; P=0.58$ ). However, a moderate correlation level between MSD and technique mastering level for both, right $(r=0.66, P<$ $0.005)$ and left side $(r=0.72 ; P<0.002)$. Even though muscular strength is an important factor for capoeira practitioners, the results suggested that the technique mastering level has a higher impact. The MSD seems to play an important role in training planning in order to obtain optimal results. Maximizing the effectiveness of this capoeira movement may depend more on the speed, not on strength level. They also suggest the need training over all the neuromuscular system (NMS) in order to improve intra and intermuscular coordination of fast-twitch muscular fibres.

\section{D2.P13. Supervised physical activity and foetal} heart rate response in pregnant smokers

\section{OLGA ROLDAN-REOYO ${ }^{1 *}$, RUBEN BARAKAT ${ }^{2}$, MIREIA $^{2}$ PELAEZ ${ }^{3} \&$ LINDA MAY ${ }^{4}$}

\section{${ }^{1}$ Swansea University, ${ }^{2}$ Technical University of Madrid, ${ }^{3}$ European University of The Atlantic, ${ }^{4}$ East Carolina University}

*Corresponding author:

olga.roldanreoyo@swansea.ac.uk

@camotilllo

Foetal chronic hypoxic stress is associated with maternal smoking (Bush et al., 2000, Placenta, 21(8), 824-833) which might affect foetal heart development (Paradis et al., 2015, PLOS, 10(2),1-21) and result in lower foetal heart rate (FHR) (Kapaya et al., 2014, The Journal of Maternal-Fetal \& Neonatal Medicine Dec(15), 1-5). Physical activity (PA) during pregnancy has been used as a tool for smoking cessation during pregnancy, even though, PA has not been effective for behavioural change in pregnant women who continued smoking (Ussher et al., 2015, Health Technology Assessment, 19(84), 1-135). However, PA during pregnancy has a positive effect in foetal heart development and autonomic nervous system function (May et al., 2010, Early Human Development, 86(4), 213-7) which might mitigate the harmful effect of smoking during gestation. Therefore, the aim of this work was to evaluate FHR response in smoking pregnant women who attended a PA programme during pregnancy. Eighty-one pregnant women were enrolled in the study and allocated into Exercise Group (EG) or Control Group (CG). All participants signed an informed consent and the study was approved by institutional Ethics Committee. EG participants attend a PA programme three times a week. Programme sessions lasted for $60 \mathrm{~min}$ and the intensity was moderate. CG participants followed their normal obstetrical care. All participants in the study performed a FHR assessment protocol between 34-36 weeks of gestation, the protocol consisted in a $3 \mathrm{~min}$ walking test at $40 \%$ intensity using Karvonen formula, participants were asked to fast for $90 \mathrm{~min}$ prior the protocol. For the present work only data from pregnant smokers have been analysed based on cigarettes smoked per day $(E G=4.2 \pm 2.4$ vs $C G=5.8 \pm 2.6 ; P>0.05)$. Seven pregnant women (EG) and 4 pregnant women (CG) were analysed. All participants delivered healthy offspring, no statistical differences between groups (gestational age at birth, birth weight, height, head circumference and Apgar score; $P>0.05$ ). Results showed no statistical differences in resting FHR (EG $=145.7 \pm$ 14.3 beats $/ \mathrm{min}$ vs $C G=137.5 \pm 8.7$ beats $/ \mathrm{min} ; P>0.05)$ and FHR after maternal effort $(150.1 \pm 17.3$ beats/min vs $C G=143.3 \pm$ 15.7; $P>0.05)$. Significant differences were found in FHR recovery time after maternal effort $(E G=2 \min 17 \mathrm{~s}$ vs $C G=7 \mathrm{~min} 17 \mathrm{~s}$; $P<0.05)$. Although smoking during pregnancy is harmful for 
foetal heart development, PA might have a protective effect evidenced by results in foetuses from EG, indicators the foetus is normoxic. These findings are evidence that foetuses of exercisers are more likely to be normoxic as well as enhanced cardiac autonomic nervous system function. However, extrapolate this results is difficult due to our small sample.

D2.P14. The associations between diabetes and both cardiovascular disease and all-cause mortality are modified by grip strength: evidence from UK Biobank prospective population-based cohort study

ANNE SILLARS, HUI LI, FANNY PETERMANN, NAVEED SATTAR, JILL PELL, JASON GILL, STUART GRAY \& CARLOS CELIS-MORALES*

\section{University of Glasgow \\ *Corresponding author: carlos.celis@glasgow.ac.uk @carloscelism}

Both grip strength and Type 2 diabetes (T2D) are predictors of mortality and cardiovascular disease (CVD) (Celis-Morales et al., 2016, European Heart Journal, 38(2), 116-122; Ntuk et al., 2017, Diabetic Medicine, 34(8), 1120-1128). People with diabetes are known to have lower grip strength, but it is not known whether these risk factors interact in predisposing to adverse health outcomes. The aim of this study was to determine the interactions between T2D and grip strength and their association with health outcomes. We undertook a prospective, general population cohort study using data from the UK Biobank. Cox proportional hazard models were used to explore the associations between both grip strength and T2D and the outcomes of all-cause mortality and CVD incidence/mortality, and to test for interactions between diabetes and grip strength. Grip strength was measured using standardised protocols across all 22 UK Biobank centres. Low and High levels of grips strength (expressed in $\mathrm{kg}$ ) were defined using age and sex-specific median as cut-off point. The UK Biobank study was approved by the North West MultiCentre Research Ethics Committee and all participants provided written informed consent to participate in the UK Biobank study. Of 498,348 UK Biobank participants, 24,745 (5.0\%) had selfreported diabetes at baseline. Over a median follow-up of 7.0 years [IQR 6.3 to 7.7], 6,545 participants developed CVD and 14,148 (2.8\%) died; 980 from CVD. Type 2 diabetic participants were at higher risk of all-cause and CVD mortality and CVD incidence. There were significant interactions whereby the risk of all-cause mortality was higher in participants with diabetes with low grip strength (HR: $2.38[2.14 ; 2.64])$ than high grip strength (HR: 1.25 [1.11; 1.42]). Participants with diabetes were only at significantly increased risk of CVD incidence and mortality if they also had low grip strength. The risk of adverse health outcomes among diabetic patients may be attenuated in those with high grip strength. Low grip strength may be useful to identify a higher risk sub-group of diabetes patients. Intervention studies are required to determine if resistance exercise can reduce risk.
D2.P15. Fundamental motor skill proficiency of the UK child population during early childhood and middle childhood, while considering weight status and ethnicity

\section{LEANNE JAYE WALKER*, MICHAEL DUNCAN \& EMMA EYRE}

\section{Coventry University}

*Corresponding author: leanne.jaye.walker@gmail.com

During childhood, physical activity (PA) participation is essential for good physical, psychological and social development (Janssen \& LeBlanc, 2010, International Journal of Behavioral Nutrition and Physical Activity, 7, 40). Fundamental Motor Skills (FMS) proficiency is observed as a facilitating and obstructive factor to PA engagement (Lubans et al., 2010, Sports Medicine, 40(12), 1019-1035); with FMS proficiency as a precursor and consequence of childhood weight status (Robinson et al., 2015, Sports Medicine, 45(9), 1273-1284). PA engagement levels differ between ethnic groups along with weight status (Eyre \& Duncan, 2013, ISRN: Obesity) and FMS proficiency has been found to differ between countries (Bardid et al., 2016, Acta Paediatrica, 105 (6), e281-e290). Few UK based studies have explored FMS proficiency while also considering ethnicity. There are also a lack of UK studies addressing specific time periods where children are expected to have acquired FMS (early childhood; EC) and undergone the development stage (middle childhood; MC); as in the UK PE curriculum. The study aimed to examine FMS proficiency in EC and MC while considering ethnicity and controlling for body mass index (BMI) and maturation. Following institutional ethical approval and parental informed consent, 192 UK children in EC $(51 \%$ male, $49 \%$ female; mean age $\pm S D=4.9 \pm 0.27$ years) and 122 children in MC (54\% male, $56 \%$ female; $9.6 \pm 0.49$ years) participated in the study. Height $(\mathrm{cm})$ and body mass $(\mathrm{kg})$ were measured using Stadiometer (Seca 213, Germany) and scales (Tanita BF305, Tanita Inc, Japan). BMI was calculated $\left(\mathrm{kg} / \mathrm{m}^{2}\right)$. FMS was assessed using the Test of Gross Motor Development-2 (Ulrich, 2000, Examiner's Manual). Analysis of Covariance indicated a sex (boys vs. girls) by developmental stage (EC vs. MC) interaction, for object control and total FMS proficiency. Boys and girls in MC were significantly more proficient compared to EC children of the same sex $(P<$ $0.05)$. Children in $M C$ demonstrated significantly greater proficiency than EC children across all skills $(P<0.01)$. Sex disparities were shown in object control and total FMS proficiency of MC children; boys presented significantly greater proficiency than girls $(P>0.05)$. There was no significant difference in FMS between ethnic groups, nor were BMI or maturation significant covariates (both, $P>0.05$ ). UK children are progressing through the PE curriculum although sex disparities persist. Where controllable, environments facilitating a sex disparity reduction, focused towards object control skills for girls, may potentially improve the reduced PA engagement of MC girls; subsequently improving health across the lifespan. 
D2.P16. The effects of high intensity interval training on body composition for health risk reduction in obese Saudi Arabian male university students

\section{PHILIP WATKINS ${ }^{1,2^{*}}$}

\section{${ }^{1}$ Sports Performance Consultancy, ${ }^{2}$ King Fahd University of Petroleum and Minerals \\ *Corresponding author: \\ phil@sportsperformanceconsultancy.co.uk @SPCEducation}

Saudi Arabia has one of the highest levels of obesity in the world, with $75 \%$ of Saudi adults overweight/obese and an estimated 20,000 obesity-related deaths per year (AI Naami, 2016, Saudi Journal of Obesity, 4, 59-67). High-intensity interval training (HIIT) is a time-efficient strategy for inducing favourable metabolic adaptations in healthy and diseased cohorts; however few studies have evaluated its impact within obese populations (Kelly, Xenophontos, King, \& Nimmo, 2017, BMC Obesity, 4:17, 1-12). Therefore, the aim of this intervention was to evaluate the effects of a 4 week HIIT weight management programme on body composition in obese, Saudi Arabian males. With ethics approval and informed consent, 30 obese Saudi Arabian male University students (mean age: $18.5 \pm 0.6$ years; stature: $1.72 \pm 0.1 \mathrm{~m}$; body mass: $108.1 \pm 12.4 \mathrm{~kg}$; body mass index: $36.5 \pm 2.9 \mathrm{~kg} /$ $\mathrm{m}^{2}$ ) (mean $\pm \mathrm{s}$ ) with no previous resistance exercise experience or contraindications registered for this optional weight management curriculum-based programme. Following baseline measures (bioelectrical impedance analysis (BIA): Camry EF921) and a familiarisation session, participants performed HIIT consisting of 10 bodyweight exercises with maximal effort, administered as a group circuit, 2 times per week, $48 \mathrm{~h}$ apart for 4 weeks. Work-rest ratios were periodised (undulating variation), exercise selection and order remained constant, and session rate of perceived exertion (sRPE) values were recorded $30 \mathrm{~min}$ after each workout. Paired sample t-tests and effect sizes analysed pre- and post-intervention data, and a two-way repeated measures ANOVA tested for differences in SRPE. Significant reductions in body mass $(P<0.01$; Effect Size: 0.42$)$, body mass index ( $P$ $<0.01$; effect size: 0.44$)$, total fat mass $(P=0.02$; effect size: $0.39)$ and fat mass index $(P=0.02$; effect size: 0.36$)(P<0.05)$ were found. There were no significant changes in SRPE or percent body fat (PBF), however a reduction in the PBF prepost mean difference (percent body fat: $0.6 \pm 0.2 \%$ ) (mean \pm s) $(P>0.05)$ was observed. Results suggest 4 weeks of HIIT may facilitate weight loss for health risk reductions as part of an effective long-term weight management strategy in Saudi Arabian young men. However, practitioners should embrace alternative methods of enquiry to evaluate influences of culture, values and societal norms on programme effectiveness. Understanding the environment is a key skill that will enhance professional practice.

D2.P17. Effects of quadriceps strength asymmetry on stair negotiation

ABIGAIL PAGE*, CALLUM OSLER \& TOM OUTRAM

University of Derby
${ }^{*}$ Corresponding author: A.Page@derby.ac.uk

Stair negotiation is among the most challenging and hazardous types of locomotion (Roys, 2001, Applied Ergonomics, 32(2), 135-139). When exposure is taken into account, stairs are a common location for falls. Furthermore, asymmetry of leg strength has also been found to impair gait and increase fall risk. Therefore, the aims of this study were (a) to assess quadriceps strength asymmetry in healthy young adults and (b) to examine the biomechanical impact of this asymmetry on stair negotiation. With institutional ethics approval, nine participants (5 men and 4 women; age: $21 \pm 2$ years; stature: $1.78 \pm 0.10 \mathrm{~m}$; body mass: $88 \pm 20 \mathrm{~kg} ; \mathrm{BMI} 27 \pm 7$; mean \pm SD) completed at least five isometric maximal voluntary contractions of the quadriceps to assess strength asymmetry. Participants then completed twelve stair negotiation trials ( 6 ascent, 6 descent; equal numbers of trials leading with the stronger and weaker leg) on a three-step staircase comprising three force plates and surrounded by a twelve-camera motion capture system. Thirty-nine reflective markers were placed according to the Vicon Plug-in-Gait fullbody model. Vertical ground reaction forces (GRF) and wholebody centre of mass (CoM) were calculated. Results showed that quadriceps strength asymmetry ranged from $1.8-29.9 \%$ and was, on average, $13.1 \pm 10.0 \%$. However, when comparing weaker and stronger legs, results show there to be no significant difference in the GRF loading slope (weaker vs stronger; ascent:

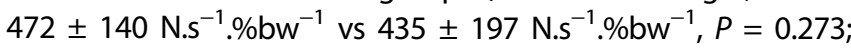
descent: $932 \pm 295 \mathrm{~N}^{-1} \mathrm{~s}^{-1} \% \mathrm{bw}^{-1}$ vs $1013 \pm 284 \mathrm{~N}^{-1} \mathrm{~s}^{-1} \% \mathrm{bw}^{-1}, P=$ 0.099), GRF unloading slope (ascent: $-1009 \pm 246 \mathrm{~N} \cdot \mathrm{s}^{-1} \% \mathrm{bww}^{-1}$ vs $-989 \pm 252 \mathrm{~N} . \mathrm{s}^{-1} . \% \mathrm{bw}^{-1}, P=0.677$; descent: $-543 \pm 123 \mathrm{~N} \cdot \mathrm{s}^{-1} . \%$ $\mathrm{bw}^{-1}$ vs $-592 \pm 165 \mathrm{~N} \cdot \mathrm{s}^{-1} \% \mathrm{bw}^{-1}, P=0.298$ ), or medio-lateral CoM range (ascent: $117 \pm 30 \mathrm{~mm}$ vs $116 \pm 18 \mathrm{~mm}, P=0.926$; descent: $106 \pm 22 \mathrm{~mm}$ vs $96 \pm 23 \mathrm{~mm}, P=0.352$ ). These results suggest that there are no biomechanical effects of quadriceps strength asymmetry on stair negotiation in a young, healthy adult population. This is likely due to relatively low strength asymmetry levels, on average. However, recommendations for future research include the study of individuals who demonstrate greater leg strength asymmetry, for example, older adults.

\section{D2.P18. Barriers to physical activity for sexual minority women aged 18-25 living in the United Kingdom}

\section{MIRANDA WESTON* \& AMBER MULCAHY}

\section{King's College London \\ *Corresponding author: miranda.weston@kcl.ac.uk}

Previous research suggests that sexual minority women (SMW) face specific barriers to physical activity in addition to those reported by other populations of women (Brittain et al., 2006, Women \& Health, 43, 75-92). Research in this area is limited to the experiences of homosexual women, rather than women identifying as other minority sexualities, and findings are predominantly from the United States. Therefore the purpose of this study was to identify the factors affecting physical activity, and how they are of influence, for SMW aged 18-25 living in the United Kingdom. A mixed methods explanatory design was adopted, comprising a 16-item questionnaire followed by semi-structured interviews. With institutional ethics approval and informed consent from 
all participants, self-identifying SMW completed the questionnaire $(N=100)$ and individuals were selected from the pool of respondents to participate in semi-structured interviews ( $N=10)$. Using Likert-type scales to investigate general and SMW-specific barriers, questionnaire respondents reported their participation in physical activity and perceptions of how various factors influenced it. The interviews were audio-recorded and analysed using grounded theory analysis, grouping data into themes. When considered as a cohort, SMWs reported the most influential factors to be time, motivation, and tiredness. However, when considering those who had experienced homophobia in isolation, 'methods of addressing homophobia and sexism in physical education and sport' was reported as the most influential barrier. $47.0 \%$ of participants $(\mathrm{N}=47)$ indicated that they had experienced homophobia during physical activity and, of that number, $68.09 \%(N=32)$ stated that this had affected their future participation. The latter group reported a lower average hours of physical activity, excluding walking, in the past week $(2.6 \mathrm{~h})$ compared to $3.54 \mathrm{~h}$ in the rest of the cohort. This was statistically significant $(P=0.048)$. Some of the common barriers identified following interview data analysis include: (1) navigating the intersecting identities of being both a woman and non-heterosexual; (2) discomfort in same-sex changing rooms due to sexual minority status; (3) the isolation associated with a 'masculine' appearance and subsequent assumptions about the individual's sexuality; and 4) discomfort within a physical activity setting with men. One participant commented, "The locker rooms can be a hostile place for a gay person ... I've experienced the worst homophobia in football". In conclusion, these results suggest an oversight when assessing the needs of SMW in sport and physical activity settings, associated with their experiences as a minority group, and should be a focus for future research. 


\section{Day 2. Posters - Physiology and Nutrition}

\author{
D2.P19. Salivary testosterone and cortisol \\ relationships in healthy adults under stress and \\ non-stress conditions: a within-participant \\ approach
}

\section{BLAIR CREWTHER ${ }^{1 *}$, LIAM KILDUFF ${ }^{2}$, CHARLIE FINN ${ }^{2,3}$, PHIL SCOT ${ }^{4} \&$ CHRISTIAN COOK ${ }^{5}$ \\ ${ }^{1}$ Institute of Sport, National Research Institute, ${ }^{2}$ A-STEM Research Centre, Swansea University, ${ }^{3}$ University of the West of Scotland, ${ }^{4}$ England and Wales Cricket Board, ${ }^{5}$ UCRISE, Canberra University \\ *Corresponding author: blair.crewther@gmail.com}

The steroids testosterone and cortisol are often viewed as having antagonist properties, whereby the stress-induced release of cortisol may dampen testosterone activity. However, positive between-participant relationships between these hormones have been reported under physical (Crewther et al., 2017, European Journal of Sport Science, 17, 188-194) and psychological stress (Maestripieri et al., 2010, Stress, 13, 413-424), which could signify complimentary actions to maintain homeostasis. To verify this interaction, we examined the salivary testosterone and cortisol relationships in healthy adults under stress and non-stress conditions, but from a within-participant perspective. With ethical approval, 12 males (age: 24.5 \pm 5.3 years) and 14 females (age: $23.5 \pm 3.9$ years) randomly completed three treatments on different days; (1) 5 $\times 10$ s cycling sprints combined with viewing a 5 -min video with "aggressive" content (S-V), (2) the same stressors in reverse order (V-S), and (3) a control session with no stimuli. Six saliva samples were taken across treatment for hormone determination, equating to a 1-hour sampling period. When data from both sexes were combined, testosterone was higher (Effect size [ES] difference $=0.4, P=0.03$ ) in the S-V session $\left(410 \pm 232 \mathrm{pmol} \cdot \mathrm{L}^{-1}\right)$ versus the control session $\left(359 \pm 173 \mathrm{pmol} \cdot \mathrm{L}^{-1}\right)$, but neither differed from the $\mathrm{V}$-S treatment $\left(383 \pm 188 \mathrm{pmol} \cdot \mathrm{L}^{-1}\right)$. No treatment effect on cortisol was identified (ES differences $=0.1$ to $0.2, P=0.161$ ) when comparing the S-V $\left(5.38 \pm 2.93 \mathrm{nmol} \cdot \mathrm{L}^{-1}\right), \mathrm{V}-\mathrm{S}(5.09 \pm$ $\left.3.58 \mathrm{nmol} \cdot \mathrm{L}^{-1}\right)$ and control sessions $\left(4.79 \pm 2.73 \mathrm{nmol} \cdot \mathrm{L}^{-1}\right)$. Within-participant modelling revealed positive testosterone and cortisol relationships $(P<0.01)$ in males across the S-V $(r=0.49), V-S(r=0.81)$ and control $(r=0.36)$ treatments. Similar correlational results $(P<0.01)$ were demonstrated within females under each respective condition $(r=0.68, r$ $=0.67, r=0.61$ ). The finding of positive relationships (within a person) between salivary testosterone and cortisol in healthy adults, who presented a broad physiological range, is suggestive of covarying steroid release patterns that could initiate complimentary effects (Crewther et al., 2017).
D2.P20. Influence of cold water immersion and cherry juice on recovery following a $\mathbf{4 2 . 2} \mathbf{~ k m}$ trail run

KATHERINE PAICE*, LYGERI DIMITRIOU, LAURA WILSON, FRANK HILLS, SCOTT SINCLAIR, MARCELLA GONDEK, KEDRIC KWAN XUE BIN, ALYSSA WOOD \& EMMA COCKBURN

\section{Middlesex University \\ *Corresponding author: kat.paice@outlook.com @kat_paice}

Both cherry juice (CJ) (Howatson et al., 2009, Scandinavian Journal of Medicine and Science in Sport, 20, 843-852) and cold water immersion (CWI) (White and Wells, 2013, Extreme Physiology and Medicine, 2) are effective for recovery following strenuous exercise. Therefore, this study examined whether CWI in conjunction with $\mathrm{CJ}$ is more effective for recovery than either alone. With ethical approval 38 endurance-trained males (age 40 \pm 7 years; height $1.8 \pm 7.7 \mathrm{~m}$; mass $76.2 \pm 10.0 \mathrm{~kg}$ ) were randomly assigned to one of four interventions (placebo (PL); $\mathrm{CJ} ; \mathrm{CWl} ; \mathrm{CWI}$ and $\mathrm{CJ}(\mathrm{MIX})$ ), before completing a $42.2 \mathrm{~km}$ run. Markers of muscle damage (muscle soreness (DOMS); maximum voluntary isometric contraction (MVIC); creatine kinase (CK)), inflammation (interleukin-6 (IL-6); C-reactive protein (CRP)) and oxidative stress (protein carbonyls (PC); superoxide dismutase (SOD); Thiobarbituric acid reactive substances (TBARS)) were measured at baseline (B), and immediately (P) (IL-6, PC and SOD only), $24 \mathrm{~h}$ and $48 \mathrm{~h}$ post run. Probabilistic magnitude based inferences analyses (difference; $\pm 90 \% \mathrm{Cl}$; difference; $\mathrm{x} / \div 90 \% \mathrm{Cl}$ for blood variables which are reported as factors), for changes over time, revealed MIX was likely harmful versus PL $(-11 ; \pm 7 \%)$, CWI $(-9$; $\pm 7 \%)$ and $\mathrm{CJ}(-8 ; \pm 8 \%)$ for changes in MVIC B-24h. MIX was likely, possibly and likely harmful versus $\mathrm{PL}(1.4 ; \mathrm{x} / \div 1.5), \mathrm{CWI}(1.2 ; \mathrm{x} / \div 1.7)$ and CJ $(1.7 ; \mathrm{x} / \div 1.6)$ for CK B-48h. B-24h MIX was possibly, most likely and very likely harmful for changes in CRP versus PL $(1.8 ; \mathrm{x} /$ $\div 2.7)$, CWI $(8.2 ; \mathrm{x} / \div 2.3)$ and $\mathrm{CJ}(5.4 ; \mathrm{x} / \div 2.6)$. B-post MIX was likely harmful $(2.8 ; \mathrm{x} / \div 2.4)$ and possibly harmful $(2.0 ; \mathrm{x} / \div 2.4)$ in comparison to PL and CWI for changes in IL-6. B-48h MIX was likely, likely and very likely harmful in comparison to PL $(1.4 ; \times / \div 1.5)$, CWI (1.8; $\mathrm{x} / \div 1.6)$ and $\mathrm{CJ}(2.1 ; \mathrm{x} / \div 1.5)$ for changes in SOD. B-24h MIX was likely $(0.5 ; \mathrm{x} / \div 1.8)$ and possibly $(0.7 ; \mathrm{x} / \div 1.8)$ beneficial for TBARS in comparison to $\mathrm{PL}$ and $\mathrm{CJ}$. Independently both treatments have been found to improve recovery; together they may limit blood supply to damaged tissues and reduce some oxidative stress markers which may be necessary for an accelerated recovery. However, further investigation is needed to clarify this and any other potential mechanisms. In conclusion, combined use of $\mathrm{CWI}$ and $\mathrm{CJ}$ is detrimental to recovery, based on measures of muscle function, inflammation and oxidative stress, following a $42.2 \mathrm{~km}$ run. 
D2.P21. Assessing the impact of dietary protein on recovery indices from resistance exercise when nutrient timing is controlled for

\section{JUSTIN ROBERTS*1 ${ }^{*}$ ANASTASIA ZINCHENKO ${ }^{2,3}$, CRAIG SUCKLING ${ }^{1}$, LEE SMITH ${ }^{1}$, JAMES JOHNSTONE ${ }^{1}$ \& MENNO HENSELMANS ${ }^{3}$}

\section{${ }^{1}$ Anglia Ruskin University, ${ }^{2}$ University of Cambridge, ${ }^{3}$ Bayesian Bodybuilding Ltd. \\ *Corresponding author: justin.roberts@anglia.ac.uk @drjustinroberts}

Previous research has demonstrated that peri-exercise protein ingestion is beneficial for adaptive gains following resistance training (Cribb \& Hayes, 2006, Medicine and Science in Sports and Exercise, 38(11), 1918-1925). High protein intakes (in excess of $2.0 \mathrm{~g} \cdot \mathrm{kg}^{-1} . \mathrm{d}^{-1}$ ) have also been reported to enhance recovery indices (Hoffman et al., 2010, Amino Acids, 38(3), 771-778), contrary to evidence that no further training benefits are observed above $1.8 \mathrm{~g} \cdot \mathrm{kg}^{-1} \cdot \mathrm{d}^{-1}$ (Lemon et al., 1992, Journal of Applied Physiology, 73(188), 767-775). This study investigated the effect of total dietary protein, whilst controlling for protein timing, on recovery indices from resistance exercise. Following University Faculty Ethics Committee approval, fourteen strength-trained individuals were randomised to two 10-day dietary regimes with a protein content of $1.8 \mathrm{~g} \cdot \mathrm{kg}^{-1} \cdot \mathrm{d}^{-1}$ (PROMOD) or $2.9 \mathrm{~g} \cdot \mathrm{kg}^{-1} \cdot \mathrm{d}^{-1}$ $\left(\mathrm{PRO}_{\mathrm{HIGH}}\right)$ in a cross-over design. On three consecutive days at the end of each regime (days 8,9,10), participants performed 3 sets of squat, bench press and bent-over rows at $80 \% 1$ repetition maximum until volitional exhaustion. A $0.4 \mathrm{~g} \cdot \mathrm{kg}^{-1}$ whey protein concentrate/isolate beverage was provided $30 \mathrm{~min}$ before and after training sessions to control for nutrient timing. Recovery was assessed across days 8-10 via assessment of perceived muscle soreness, bioelectrical impedance phase angle (as a proxy measure of muscle integrity), plasma creatine kinase (CK) and tumor necrosis factor-a (TNF-a) levels prior to daily exercise; as well as repetition performance count for each exercise. A two-way repeated measures ANOVA was performed for the main analyses, with Bonferonni post-hoc pairwise comparisons where applicable. For repetition count, no significant differences were reported between conditions $(P>0.05)$. For squat performance, total repetition count was lower at day $10(19.7 \pm 1.8)$ compared to day $8\left(23.0 \pm 2.0 ; P=0.006, n p^{2}\right.$ $=0.335 ; 95 \% \mathrm{Cl}$ for difference -5.65 to -0.92 ) within $\mathrm{PRO}_{\mathrm{MOD}}$ only. Post-exercise CK concentrations significantly increased across test days $\left(P<0.001, n p^{2}=0.583\right)$ increasing from 293 $\pm 48 \mathrm{U.L}^{-1}$ to $718 \pm 140 \mathrm{U.L}^{-1}$ within $\mathrm{PRO}_{\mathrm{HIGH}}$ and from $281 \pm$ $45{\mathrm{U} . \mathrm{L}^{-1}}^{-1}$ to $656 \pm 155 \mathrm{U}^{-\mathrm{L}^{-1}}$ within $\mathrm{PRO}_{\mathrm{MOD}}$, although no differences were reported between groups. No differences for TNF- $a$ or muscle soreness were reported between groups. Phase angle was significantly greater at day 10 for $\mathrm{PRO}_{\text {HIGH }}\left(8.26 \pm 0.82^{\circ}\right)$ compared with PRO $\mathrm{MOD}\left(8.08 \pm 0.80^{\circ}\right.$, $P=0.012, n p^{2}=0.237 ; 95 \% \mathrm{Cl}$ for difference -0.55 to -0.08$)$. Protein intake at $2.9 \mathrm{~g}^{\mathrm{kg}} \mathrm{kg}^{-1} \cdot \mathrm{d}^{-1}$ did not enhance indices of recovery following resistance exercise when peri-exercise protein consumption was controlled for. This finding suggests that daily protein intakes of $1.8 \mathrm{~g} \cdot \mathrm{kg}^{-1}$ may be sufficient for consecutive days of resistance exercise in habitually trained individuals.
D2.P22. Neuromuscular fatigue following cycling above critical power

LISA SCHÄFER*, MARK HAYES \& JEANNE DEKERLE

\author{
University of Brighton \\ *Corresponding author: L.Schafer@brighton.ac.uk
}

Critical torque is defined as the highest torque an indivudal can sustain for an indefinite period of time and has been described as a critical threshold for neuromuscular fatigue development. Similar levels of peripheral fatigue have been reported following exhaustive single limb exercise above critical torque (Burnley et al., 2012, Journal of Applied Physiology, 113, 215-223). Whether similar findings are observed after whole body exercise remains to be investigated. Therefore, the aim of this study was to compare the magnitude of neuromuscular fatigue, and in particular, the role of peripheral mechanisms in the aetiology of fatigue during cycling above critical power (CP). With institutional ethics approval, 12 healthy male participants (mean \pm SD age: $23 \pm 4$ years; $\left.V O_{2 \text { peak }}: 52.2 \pm 9.0 \mathrm{ml} \cdot \mathrm{kg}^{-1} \cdot \mathrm{min}^{-1}\right) \mathrm{com}$ pleted an incremental cycling test to determine $\mathrm{VO}_{2 \text { peak }}$ 4-5 constant-load trials to task failure to calculate CP and three randomly assigned experimental trials above $\mathrm{CP}$ where power was set to fully deplete the anaerobic work capacity (AWC) in $3(\mathrm{P}-3 \times 1)$ and $12 \mathrm{~min}(\mathrm{P}-12 \times 2)$, respectively. $\mathrm{P}-12$ was performed twice to quantify absolute reliability. Pre- and post-exercise, neuromuscular function assessment of the right knee extensors was completed to measure maximal voluntary contraction (MVC), potentiated twitch force (POT) and doublet twitch forces at $10 \mathrm{~Hz}$ (PS10) and $100 \mathrm{~Hz}$ (PS100) using femoral nerve stimulation. Voluntary activation (VA) was calculated using the interpolated twitch technique. Two-way repeated measures ANOVA were used to test for differences in condition and time. Neuromuscular measures showed good between-day reliability post-exercise with coefficient of variations ranging from 5 to $8 \%$ (95\% lower LoA expressed as percent change from pre-exercise: $-16 \%$, $-23 \%,-21 \%,-16 \%,-31 \%$ for MVC, POT, PS10, PS100 and VA, respectively). Cycling above $C P$ induced neuromuscular fatigue (MVC: $-19 \pm 8 \%$ for P-3 vs $-16 \pm 6 \%$ for P-12) composed of both peripheral fatigue (POT: $-36 \pm 12 \%$ vs $-31 \pm 11 \%$; PS10: $-39 \pm 18 \%$ vs $-36 \pm 18 \%$; PS100: $-18 \pm 4 \%$ vs $-13 \pm 3 \%$ for P-3 and P-12, respectively), and central fatigue (VA: $-12 \pm$ $3 \%$ for $\mathrm{P}-3$ and $-13 \pm 3 \%$ for $\mathrm{P}-12)$, with no significant difference $(P>0.05)$ between $\mathrm{P}-3$ and $\mathrm{P}-12$ post-exercise in each recorded measure (MVC: $d=0.09$; POT: $d=0.16$; PS10: $d=-0.03$; PS100: $d=0.26$; VA: $d=-0.10$ ). The results indicate that the magnitude of neuromuscular fatigue observed following full depletion of AWC, is not dependent upon the power selected above CP.

D2.P23. Lutein and Zeaxanthin: do they influence visual performance in athletes?

PARIMALA SIVAPERUMAN ${ }^{1 *}$, NUR SHAKIRAH B. ROOHAIZAD ${ }^{2}$, JEI NI SIM ${ }^{2}$, SYAKIRAH B. MUSATAFA ${ }^{2}$, CHONG HAO NATHANAEL ONG ${ }^{1}$, HONG EN JOSHUA CHUA $^{1}$ \& RICHARD SWINBOURNE ${ }^{1}$ 
${ }^{1}$ Sport Singapore, ${ }^{2}$ Temasek Polytechnic

*Corresponding author:

Parimala_sivaperuman@sport.gov.sg

Visual function can be enhanced via an increased intake of dietary lutein and zeaxanthin (L\&Z). Participants in batting sports involving exposure to intense blue light conditions may experience eye damage, thus affecting their visual processing speed of fast balls and subsequent motor reaction times (Hammond \& Fletcher, 2012, American Journal of Clinical Nutrition, 96, $1207 \mathrm{~S}$ $-1213 S)$. Therefore, the aim of this study was to assess the dietary intake of $L \& Z$ and their correlation with visual and motor reaction times in batting sport athletes and non-athletes. Upon ethical approval, 23 athletes (mean age: $22.1+4.3$ years) and 9112: 30 non-athletes (mean age: $21.3+2.6$ years) completed a semi-quantitative, validated food frequency questionnaire (FFQ) (Bassett et al., 2016, Public Heath Nutrition, 19, 2357-2368) to assess dietary L\&Z intake over the preceding 12 month period. The FFQ was accompanied by validated tests for reaction time (RT); (the time retinal cells detect a visual stimulus and send electrical signals to the visual cortex) and motor time (MT); (the time needed for the neuromuscular system to send information to the muscles) (Schuhfried, 2012, Vienna test system: Psychological assessment. Moedling: Schuhfried). On average, athletes consumed $29 \%$ more L\&Z compared to nonathletes. A moderate but unclear effect size was observed between groups for dietary L\&Z intake $(d=0.47, \pm 0.5,9125$ : $P=0.25)$. Compared to non-athletes, athletes recorded a moderate but unclear faster RT $(d=0.58, \pm 0.82, P=0.01)$. No significant difference was observed in MT between groups $(P=$ $0.86)$. Small $\left(r_{s}=-0.107, P=0.628\right)$ and moderate $\left(r_{s}=-0.423, P=\right.$ 0.44 ) correlations between L\&Z intake and RT and MT were observed among athletes, respectively. Thus, it is suggested that in athletes a higher intake of $L \& Z$ is associated with a faster RT and MT. Conversely, a high intake of L\&Z was associated with a slower RT and MT in non-athletes $\left(r_{s}=0.165, P=0.38\right)$ and $\left(r_{s}=\right.$ $0.113, P=0.55)$. Though unclear, this study showed trends that athletes consumed higher intakes of $L \& Z$ and possessed a faster visual performance time, compared to non-athletes. A relationship between the dietary intake of $L \& Z$ and visual and motor reaction times among athletes may exist. This study adds to the limited body of visual performance research in athletes, and may suggest a competitive advantage that is currently underutilised. Further research, involving a controlled $L \& Z$ intervention between athlete populations, is warranted.

D2.P24. Effect of sodium bicarbonate ingestion on intermittent running and hockey specific skill

\section{CAROLINE SUNDERLAND* \& DAVID MACUTKIEWICZ}

\section{Nottingham Trent University \\ *Corresponding author: caroline.sunderland@ntu.ac.uk}

Research has demonstrated that bicarbonate supplementation can enhance repeated cycle or running sprints, which take place within team sports interspersed with walking, jogging and running. Despite, the extensive research completed relating to sodium bicarbonate supplementation, to date, no studies have investigated whether bicarbonate supplementation improves team sport specific running and skill, which is often the determinant of match outcome. Thus, the aim was to examine the effect of sodium bicarbonate supplementation on team-sport running and skill performance. Following ethical approval and informed consent, 8 elite female field hockey players (age $23 \pm 5 \mathrm{y}$, body mass $62.6 \pm 8.4 \mathrm{~kg}$, height $1.66 \pm 0.05 \mathrm{~m}$ ) completed three Field Hockey Skill Tests (FHST) interspersed with four sets of the Loughborough Intermittent Shuttle Test. Between $3 \mathrm{~h}$ and 90 min prior to exercise, participants were supplemented with $0.2 \mathrm{~g} \cdot \mathrm{kg}^{-1}$ body mass (BM) of a placebo (maltodextrin) or $0.3 \mathrm{~g} \cdot \mathrm{kg}^{-1} \mathrm{BM}$ sodium bicarbonate. The FHST required participants to dribble round cones in a specified order before shooting in a goal, the area of which was randomly determined by the switching on of a light. Field hockey skill performance incorporated overall performance time (PFT), movement time (MT), decision-making time (DMT), and penalty time (PT). Sprint time (ST), blood lactate (YSI, Stat 2300 Plus, USA), bicarbonate, pH (Radiometer Ltd, ABL 700, UK) and base excess were measured throughout. Data (mean \pm SD) were analysed using a two-way analysis of variance (ANOVA) with repeated measures (trial $x$ time), with Cohen's $d$ effect sizes used to interpret the magnitude of differences. Bicarbonate concentration and $\mathrm{pH}$ were greater during the bicarbonate trial compared with the placebo (both $P<0.001$ ). Bicarbonate did not alter PFT (placebo: $87.9 \pm 6.9 \mathrm{~s}$; bicarbonate: $89.0 \pm 7.8 \mathrm{~s}, P=0.544, \mathrm{~d}=0.16$; range -11.3 to $7.2 \%$ improvement), MT, DMT, PT or ST (placebo: $2.87 \pm 0.12 \mathrm{~s}$; bicarbonate: $2.86 \pm 0.12 \mathrm{~s}, P=0.893, \mathrm{~d}=0.09)$. RPE was lower during the bicarbonate condition $(P=0.021, \mathrm{~d}=0.48$; placebo: $13 \pm 2$; bicarbonate: $12 \pm 2$ ). Acute ingestion of bicarbonate did not improve sprint or sport specific skill performance. The inter-individual variability in response to bicarbonate (Saunders, Sale, Harris, \& Sunderland, 2014, International Journal of Sports Physiology and Performance, 9, 627-632) may partially explain the lack of a performance improvement in the current study. Bicarbonate ingestion did result in a lower perception of effort during team sport running, which may be important in a competitive match situation.

D2.P25. The effects of low and moderate dose caffeine supplementation on upper and lower body maximal voluntary concentric and eccentric muscle force

\section{JASON TALLIS*, HARLEY YAVUZ \& MICHAEL DUNCAN}

\section{Coventry University}

*Corresponding author: tallisj2@uni.coventry.ac.uk @jason_Tallis

A review of the literature has highlighted that the performance enhancing effect of caffeine on muscular strength may be greater in larger and lower body muscle groups (Warren et al., 2010, Medicine and Science in Sport and Exercise Science, 42, 1375-1387). Despite this, there is a distinct dearth of data that directly explores differences in erogenicity between upper body and lower body musculature. As such, the present study sought to investigate the effects of low and moderate doses of caffeine on the maximal voluntary strength of the elbow flexors and knee extensors. Following institutional ethics approval and completion of informed consent, ten apparently healthy, recreationally active, but non-specifically 
strength trained males (Mean \pm SE: Age: $21 \pm 0.3$ years; height: $176 \pm 2.1$; body mass: $73.9 \pm 3.4$ ) agreed to participate in the study. Participants were low habitual caffeine users (Mean \pm SE; $122 \pm 40.9 \mathrm{mg} /$ day) as identified by the completion of a caffeine consumption questionnaire. Using a randomised, counterbalanced and double blind procedure, isokinetic concentric and eccentric strength of the elbow flexors and the knee extensors was measured at 60 and $180 \mathrm{deg} / \mathrm{s} 60 \mathrm{~min}$ following consumption of a placebo, $3 \mathrm{mg} \cdot \mathrm{kg}^{-1}$ body mass caffeine and $6 \mathrm{mg} \cdot \mathrm{kg}^{-1}$ body mass caffeine. Assessments were separated by at least $48 \mathrm{~h}$ and were conducted at the same time of day. Repeated measures ANOVA revealed that there was no effect of caffeine on the maximal voluntary concentric and eccentric strength of the elbow flexors, or the eccentric strength of the knee extensors ( $P>0.05$ in each case). 3 and $6 \mathrm{mg} \cdot \mathrm{kg}^{-1}$ body mass caffeine caused an increase in peak concentric force of the knee extensors at $180 \mathrm{deg} / \mathrm{s}$. These differences were approaching significance with the lower caffeine dose $(P=0.08 ; d=0.68)$, but reached significance with the higher caffeine dose $(P=0.03 ; d=0.83)$. No difference was apparent between the two concentrations $(P=0.64 ; d=0.09)$. Only $6 \mathrm{mg} \cdot \mathrm{kg}^{-1}$ body mass caused an increase in peak concentric force during repeated contractions of the knee extensors which was approaching significance $(P=0.06 ; d=0.47)$. These findings demonstrate that relatively low dose caffeine treatment may be effective in improving muscular strength in nonspecifically trained individuals, but only in larger muscle groups of the lower limb. There was no clear dose response effect, however the results infer that the effective caffeine concentration to evoke improved muscle performance may be related to contraction type.

\section{D2.P26. Effect of the re-booting cognition} technique on blood lactate concentration during the post-exercise period

\section{ALYX TAYLOR $^{1 *}$, CHRISTIN MASKUS $^{2} \&$ VIJAY PABBATHI}

${ }^{1}$ AECC University College, ${ }^{2}$ American Chiropractic Centre ${ }^{*}$ Corresponding author: ataylor@aecc.ac.uk

The Re-Booting Cognition (RBC) technique is the simultaneous use of pulse and extended exhalation as a process for speeding recovery for optimum performance in sport. The participant concentrates on their own pulse while carrying out breathing and relaxation exercises (Pabbathi, 2010, Pulse-Mediated relaxation: The secret of using pulse to achieve relaxation. Bournemouth, Dorset; Pabbathi, 2017, Winning begins through recovery. Bournemouth, Dorset). The study was designed to investigate the effect of using the RBC technique for 7 min during recovery from intense exercise on the blood lactate concentration and perceived relaxation of healthy participants. Thirty healthy students, mean age: 25.00 (SD 5.08), range: 19-42 years, took part. The study group $(n=15)$ participants were trained in the RBC technique and the control group $(n=15)$ participants were not trained prior to the experiments. Each participant cycled for 5 min with 4 min warm up at approx $50 \mathrm{rpm} 0.5 \mathrm{Kp}$ then the final min at maximum rpm possible. Perceived exertion (RPE; Borg
Scale) was recorded immediately after. The study group then practised $\mathrm{RBC}$ for 7 min and the control group sat quietly for the same period of time. Blood samples were taken before and after the intervention. All participants were asked to report their observations during recovery and the study group used a 5 point Likert scale to evaluate the effect of the RBC. There was a statistically significant decrease in mean blood lactate concentration in the study group from $10.03 \mathrm{mmol} / \mathrm{I}$ (SD 1.70) to $8.64 \mathrm{mmol} / \mathrm{I}(\mathrm{SD} 1.65)(P=0.031)$ after using RBC. The control group showed no significant change in lactate from $9.59 \mathrm{mmol} / \mathrm{l}$ (SD 2.05) before to $9.68 \mathrm{mmol} / \mathrm{l}$ (SD 1.71) after sitting quietly for $7 \mathrm{~min}$. Blood lactate concentration post exercise was significantly related to perceived exertion measured on the Borg scale, $r_{s}=.44$, $95 \% \mathrm{BCaCl}[.743, .021], P=008$. All participants in the test group reported feeling relaxed during the use of RBC. These results suggest that the use of the $\mathrm{RBC}$ technique during the post exercise period can increase perceived relaxation and may help reduce the concentration of blood lactate. The controlled breathing of the $\mathrm{RBC}$ technique may have a role in attenuating the sympathetic signalling during the recovery.

\section{D2.P27. Contributions of knee extensor moment arm and quadriceps femoris muscle volume on knee extensor strength in children}

\section{NOBUAKI TOTTORI, TADASHI SUGA, MIYUKI HORI, TADAO ISAKA \& SATOSHI FUJITA}

\section{Ritsumeikan University \\ *Corresponding author: sh0037xp@ed.ritsumei.ac.jp}

Muscle strength, measured as the joint torque, is an important factor for performance in both activities of daily living and sporting events. Joint torque has previously been determined not only by muscle size but also moment arm (MA) (Baxter \& Piazza, 2014, Journal of Applied Physiology, 116, 538-544). However, it is unknown what the contributions of MA to joint torque in children. Larger increase in muscle strength compared with the increaser in muscle size was observed in adolescents (Kanehisa, Yata, Ikegawa, \& Fukunaga, 1995, European Journal of Applied Physiology and Occupational Physiology, 72, 150-156). Therefore, we hypothesized that a strong correlation would exist between MA and joint torque as same as muscle size. The aim of this study was to examine the relationship between joint torque and both MA and muscle size in the knee extensors of preadolescent boys. Twenty boys (mean \pm SD; age: $10.7 \pm$ 0.9 years; height: $142.0 \pm 8.1 \mathrm{~cm}$; body mass: $34.7 \pm 6.4 \mathrm{~kg}$ ) participated in this study with institutional ethical approval. All participants were of an age before peak height velocity as assessed by existing regression equations (Mirwald, Baxter-Jones, Bailey, \& Beunen, 2002, Medicine and Science in Sports and Exercise, 34, 4 689-694). Peak isometric and isokinetic knee extensor torques were measured by dynamometer (BIODEX system 3, BIODEX Medical). Isokinetic extension was performed over the full range of motion at angular velocities of $60 \mathrm{deg} \cdot \mathrm{s}^{-1}$ and $180 \mathrm{deg} \cdot \mathrm{s}^{-1}$. Participants performed two knee extensions for $3 \mathrm{~s}$ with 1-min rest between assessments. Quadriceps femoris anatomical cross-sectional area (ACSA), muscle volume (MV), and 
knee extensor MA were measured by magnetic resonance imaging. Knee extensor isometric torque was significantly correlated with knee extensor MA $\left(r=0.724, R^{2}=0.524\right.$, $P<0.001)$ in addition to quadriceps femoris ACSA $(r=$ $\left.0.513, R^{2}=0.263, P=0.021\right)$ and $\mathrm{MV}\left(r=0.617, R^{2}=0.381\right.$, $P=0.004)$. Knee extensor isokinetic torque was significantly correlated with knee extensor MA in addition to quadriceps femoris $A C S A$ and $M V$ at each of the two angular velocities tested. Multiple regression analysis resulted in strong correlations between knee extensor torque and both MA and quadriceps femoris MV during isometric $\left(r=0.760, R^{2}=0.578, P<0.001\right)$ and isokinetic tests. The present findings demonstrated that longer MA might be a strong contributor to generating higher joint torque in knee extensor at least as important as muscle size in children. 
Day 2. Posters - Psychology

\begin{abstract}
D2.P28. The direct and organisational stress moderating effects of perceived social support on athletic performance in elite sport
\end{abstract}

\author{
RACHEL ARNOLD ${ }^{1 *}$, THOMAS EDWARDS ${ }^{1} \&$ TIM REES $^{2}$ \\ ${ }^{1}$ University of Bath, ${ }^{2}$ Bournemouth University \\ *Corresponding author: rsa24@bath.ac.uk \\ @RachArnoldRachArnold
}

Increasingly, research is demonstrating that organisational stressors can not only be a prevalent and problematic type of stressor for athletes; but can also be experienced more than competitive demands. One model that can be used to explain the relationship between organizational stressors and outcomes is the meta-model of stress (Fletcher et al., 2006, In Hanton \& Mellalieu (Eds.), Literature reviews in sport psychology (pp. 321-373). Hauppauge, NY: Nova), which suggests that environmental stressors are mediated by certain processes (e.g., appraisal, coping) and, as a consequence, can result in various outcomes. Additionally, the model posits that various personal and situational characteristics can moderate the stress process. One moderator that has received scholarly attention in relation to competitive stress is that of social support; however, scholars have yet to examine this moderator in relation to organisational stress. The purpose of this study, therefore, was to examine the relationship between organisational stressors and perceived performance in sport, with particular focus on the potential moderating role of social support. The hypotheses were as follows: the frequency of organisational stressors will directly predict perceived performance in athletes $(\mathrm{H} 1)$; perceived social support will directly predict perceived performance irrespective of levels of stressors $(\mathrm{H} 2)$; and, perceived social support will have stress-buffering effects on perceived performance; specifically, the detrimental relationship between organizational stressors and performance would be reduced for those with high perceived support versus those with low levels (H3). With institutional ethical approval, 118 elite athletes ( 58 male; $M_{\text {age }}=20.50$ ) were recruited who competed across a total of 35 sports at a national $(n=24)$ or international level $(n=94)$. Participants completed an online questionnaire pack which measured perceived organisational stressors, social support, and athletic performance. In addition to the main effects identified (viz. $\mathrm{H} 1$ partially supported, H2 fully supported), moderated hierarchical regression analyses also found five significant interactions $(P<.05)$ which demonstrated that social support did act as a significant moderator of the relationship between organisational stressors and performance. Contrary to extant literature, however, the findings illustrated reverse buffering whereby social support exacerbated rather than mitigated athletes' stress reactions (i.e. impaired performance) when experiencing greater frequencies of organisational stressors. By discussing various explanations for reverse buffering, this study not only advances theoretical understanding of organisational stress, but also presents a number of significant implications for athletes, coaches, and applied practitioners aiming to enhance performance in pressurized and demanding situations.

\section{D2.P29. The psychological impact of injury on amateur horse riders}

\section{EMMA DAVIES* \& MERLIN PERLO}

\section{University Centre Hartpury}

*Corresponding author: Emma.Davies5@hartpury.ac.uk @Emma_Dave

The psychological and emotional responses after serious injury are known to affect an individual's well-being, as well as their likelihood of effectively and successfully returning to sport. While injuries in equestrians may be well documented, research into the short and long-term psychological implications are scant. Injured athletes were found to go through a range of negative emotions during a period of injury rehabilitation, such as: anxiety, anger, confusion, depression, impaired self-esteem, hopelessness and worry. These psychological processes have the potential to affect recovery, both physical and psychological, with a major concern being that many athletes retain an element of fear of re-injury. The aim of this study was to identify emergent common themes within the amateur rider cohort to provide an appraisal of the psychological and emotional processes. Ethical consideration was granted by the institutional ethics committee prior to data collection. Five amateur riders were interviewed about their experiences when they suffered a serious injury (defined as requiring a minimum of 21 days off), considering the psychological and emotional processes participants encountered in the period from injury to recovery. This study found that amateur riders face similar negative psychological impact after injury as in other sports, however, while some long term consequences were seen as each participant changed their riding practices after the incident, more research is needed to determine the extent to which these processes affect longevity and success within the sport. With this information an understanding can be formed of the implications for the sport and its participants, as well as potential support regarding psychological interventions and their application to equestrian sport.

D2.P30. Psychological and emotional responses of elite riders to the injury of their horses

\section{EMMA DAVIES*, JULIA ENNIS \& RICHARD COLLINS}

\section{University Centre Hartpury \\ *Corresponding author: Emma.Davies5@hartpury.ac.uk @Emma_Dave}

Sport is considered a high risk environment for athletes sustaining injury. Athletes are known to experience negative 
emotional and psychological responses to injury, which can destabilise identity. For athletes, identity is typically constructed around participation in sport and after injury, this identity can be threatened. Within equestrian sport, the addition of a partner athlete also at risk of injury presented a unique circumstance for investigation. The purpose of the present study was to investigate the emotional and psychological responses of elite riders to the injury of their horses. Ethical consideration was granted by the institutional ethics committee prior to data collection. Five riders competing internationally in dressage, show jumping and eventing were interviewed about their experiences when their horse suffered a serious, or career-ending injury. Thematic analysis revealed that depression, denial and guilt were common emotional responses; these emotions mirror those experienced by athletes when injured themselves. Injury to their horse led riders to question their identity as elite athletes, and question their careers within equestrian sport. The nature of the sport means riders are at heightened risk of the injury-related psychological stress, as riders perceive the horse as part of the identity they develop as an athlete. Coping resources for riders are needed within equestrian sport to minimise the psychological impact of injury, either of rider or horse particularly at elite level. Goal setting and social support systems are suggested beneficial for coping with injury in other environments and should be implemented within sports science support for elite riders.

\section{D2.P31. Understanding junior to senior level transition experiences: a case study of an elite rugby league academy}

\section{CHARLOTTE HINCHLIFFE*, ANDREW MANLEY, BEN JONES \& SUSAN BACKHOUSE}

\section{Leeds Beckett University \\ *Corresponding author: \\ C.R.Hinchliffe@leedsbeckett.ac.uk @SportPsychHinch}

The transition from junior to senior level has been described as the most difficult and most crucial within-career transition in sport (Stambulova et al., 2009, International Journal of Sport and Exercise Psychology, 7, 395-412). Successful transition occurs when athletes are able to effectively cope with any demands they encounter, and coping is influenced by the interaction of transition resources and barriers (Stambulova [2003]. In N. Hassmén (Ed.), SIPF Yearbook 2003 (pp. 97-109). Örebro: Örebro University Press). The purpose of the present study was to explore the demands, resources, and barriers experienced by adolescent rugby league players during the transition from junior to senior level. Following institutional ethical approval, retrospective timeline interviews were conducted with 26 players $(100 \%$ male; age $17-20$ years, mean age $=17.81$ ) from a professional academy squad. Interviews sought to elicit a data-rich description of participants' season-long experiences. To encourage players to consider holistic experiences from both within and outside of the rugby context (Wylleman \& Lavallee, 2004, In Weiss (Ed.), Developmental sport and exercise psychology: A lifespan perspective (pp. 507-527). Morgantown, WV: Fitness Information Technology), prior to interviews participants also engaged with a self-portrait task. An inductive content analysis of the data revealed that participants encountered demands in four domains of life: rugby, school/work, relationships, and maturation. Within each of these domains, demands were categorised into higher-order, lower-order and sub-themes. Resources and barriers were categorised as either internal or external to participants and were clustered under the following higher order themes: internal themes included psychological characteristics, psychological skills and individual factors; external themes included social environment factors, structural environment factors and situational factors. The specific context in which the present study was conducted (i.e. adolescent rugby league) provides a novel contribution to the literature and an insight for practitioners working in similar contexts into the experiences of athletes currently transitioning from junior to senior level. The importance of supporting athletes holistically is emphasised, including monitoring demands both within and outside of the sporting domain. An insight into situations (e.g. injury) when athletes experience particularly high numbers of demands is provided, and it is suggested extra support for athletes may be required in these circumstances. Finally, a range of external resources (e.g. social support) and internal resources (e.g. self-regulation) that facilitate effective coping are identified. These resources can be used to inform effective models of talent development and facilitative environments in which optimal development can occur.

\section{D2.P32. Antecedents of competitive state anxiety} and self-confidence among deaf male soccer players

\section{JOSEF D. BAINES ${ }^{1 *}$, RICHARD MULLEN ${ }^{2}$, COSTAS I. KARAGEORGHIS ${ }^{1}, \&$ JIM A. CROMWELL ${ }^{3}$}

\author{
${ }^{1}$ Brunel University London, ${ }^{2}$ University of South Wales, \\ ${ }^{3}$ Exeter Royal Academy for Deaf Education \\ *Corresponding author: josefbaines@yahoo.com \\ @BainesJosef
}

The competitive state anxiety response has been a subject of systematic research for over five decades (Uphill, 2016, In Lane (Ed.), Sport and exercise psychology (2nd ed., pp. 50-75). London: Routledge). Researchers have focused primarily on intervention strategies designed to help athletes cope with or reduce high levels of competitive state anxiety (Rumbold, Fletcher, \& Daniels, 2012, Sport, Exercise, and Performance Psychology, 1, 173-193). Identification of such antecedents enables sport psychologists to design effective interventions to combat the potentially deleterious effects of anxiety. In disability sport, much of the literature chronicles athletes who have physical impairments (Martin, 2013, In Hanrahan, \& Andersen (Eds.), Routledge handbook of applied sport psychology (pp. 432-440). New York, NY: Routledge). Albeit that previous research focused on disabled athletes' experiences, the results may not readily generalise to athletes with other impairments; for example, deaf/hard of hearing (deaf/hoh) athletes, who define themselves as able-bodied (Vose, Clark, \& Sachs, 2013, In Hanrahan \& Andersen (Eds.) (pp. 450-459)). Accordingly, the purpose of this study was to examine the situational antecedents of multidimensional state anxiety 
and self-confidence among male deaf/hoh soccer players. A correlational design was employed with situational antecedent variables used as predictors of state anxiety variables. With institutional ethics approval, 101 deaf/hoh males (mean $+s$ age: $25+6.0$ years; mean $+s$ soccer experience: $15.42+$ 8.9 years) completed two questionnaires $1-2 \mathrm{~h}$ prior to a match. Three situational factors, namely, perceived readiness, coach influence, and match conditions, were measured using the Pre-Match Questionnaire (Lane, Rodger, \& Karageorghis, 1997, Perceptual and Motor Skills, 84, 427-433). The three subcomponents of competitive state anxiety (self-confidence, somatic anxiety, and cognitive anxiety) were measured using the revised Competitive State Anxiety Inventory-2 (Cox, Martens, \& Russell, 2003, Journal of Sport \& Exercise Psychology, 25, 519-533). Multiple regression analyses indicated that only perceived readiness significantly predicted $(\beta=-1.68, P<0.05)$ the cognitive state anxiety experienced by deaf/hoh soccer players. Situational factors did not predict either self-confidence or somatic state anxiety. At an applied level, sport psychologists should focus their attention on pre-competition interventions that increase perceived readiness and reduce cognitive state anxiety. Future research should address the psychometric properties of the questionnaires employed in this study, and of other popular questionnaires in the sport psychology literature, for deaf/hoh athletes. Such work would serve to bolster the research base.

D2.P33. A holistic case study approach to predelivery routines in training and competition

\section{ADAM KELLY*}

\section{Southampton Solent University \\ *Corresponding author: adam.kelly@solent.ac.uk @adamfkelly}

Pre-performance routines have been consistently exhibited across a variety of closed self-paced skills. More recently, authors have suggest that elite cricket batsmen may utilise pre-delivery routines (PDR) to enhance their focus (Cotterill, 2011, Journal of Sport Psychology in Action, 2, 81-91; Barker \& Slater, 2015, The Psychologist, 28, 552-557). However, little research has explored routines holistically in both training and competition environments. This case-study consisted of two phases. The first phase aimed to explore the behavioural consistency in both environments. Second phase aimed to examine any psychological strategies employed and what functions both behaviours and psychological strategies serve within a PDR. Institution ethical approval was granted prior to data collection. The participant was a 31 year old male professional cricketer, with 10 years' professional playing experience. A two phase methodology was utilised; observation and interview. First phase, participant was video recorded in both environments and footage was analysed post-event. The participant received 110 deliveries during data collection (68 in competition and 42 in training). Second phase, the interview was conducted under an Interpretative Phenomenological Analysis approach. The interview started under a 'think aloud protocol', followed by semi-structured interview questions. The interview lasted 64 min. Data were systematically analysed through;
Immersion, Annotation, Emerging Themes and Identifying Super-Ordinate Themes. Findings revealed that behavioural consistency was higher in competition (84\%) than in training (54.7\%). Main behaviours served the same functions in both environments; setting of the feet served comfort, shuffling feet served enhancing balance and lifting bat up served to switch on focus. The participant reported utilising self-talk and attention allocation strategies. Interestingly, the self-talk phrase 'watch the ball, play straight' formed a rhythmic mantra which enabled the participant to block out distractions and provide a 'fierce' focus. However, in training the participant would add technique-based instructional self-talk phrases to improve weaknesses, although avoid these phrases in competition. Attention was allocated towards the external and distal stimuli; the ball. Interestingly, attentional focus differed in competition where fluctuation occurs, through switching focus on/off between deliveries, compared to a constant focus during training. When comparing environments the participant highlighted the difficulty of replicating the PDR in training due to external factors which are not present in competition such as, minimal time between deliveries. Furthermore, the use of technique-based self-talk in training would be occasionally experienced in competition, reducing the 'fierce' focus to a state of 'you are almost playing blind'. In summary, it appeared difficulty for the participant to replicate the PDR's in both environments. The training-competition paradigm provides practitioners with an additional challenge when developing PDRs with cricket batsmen and should be considered when working with this population.

\section{D2.P34. An investigation of athletes' life skills transfer and application experiences}

\section{KELSEY KENDELLEN* \& MARTIN CAMIRÉ}

\section{University of Ottawa}

*Corresponding author: kelsey.kendellen@uottawa.ca @KelseyKendellen

A recent review of the literature indicates that there is some evidence that the life skills (e.g., leadership, self-regulation) learned in sport can transfer and be applied in settings beyond sport (Pierce, Gould, \& Camiré, 2017, International Review of Sport and Exercise Psychology, 10, 186-211). However, this evidence is primarily based on research that has been conducted using cross-sectional designs (e.g., oneshot interviews) to retrospectively examine athletes' perceptions of life skills transfer (Turnnidge, Côté, \& Hancock, 2014, Quest, 66, 203-217). Longitudinal research is needed to study the transfer process by documenting athletes' personal accounts and feelings of transfer over time. The purpose of this study was to investigate athletes' life skills transfer and application experiences. Ethical approval was granted from the University's Research Ethics Board. Individuals that had a prolonged history of organised sport participation (i.e., minimum of five years) as children and adolescents were recruited from the University's intramural sport program. A total of 13 athletes (seven males, six females) between the ages of 18 and 24 years old (Mage $=21.77$ ) agreed to participate and were asked to write journal entries using a social networking website over a 3-month period. The 
participants were provided with guiding questions to follow when completing an entry: (a) Where did I apply this particular skill that I learned from sport? (b) Who was around me when I applied this skill? and (c) How did I apply this skill? A total of 69 journal entries were collected, with each participant writing three to eight entries $(M=5.31)$ with each entry ranging from 67 to 685 words $(M=274.30)$. The findings revealed that the participants believed that successfully applying the life skills they learned from sport in non-sport contexts helped them make positive contributions to themselves as well as the transfer/application context. For example, one participant wrote multiple entries on how applying the emotional regulation skills she learned in sport during her teaching practicum helped her manage her nerves when leading class discussions and dealing with behavioural management issues. Ultimately, this participant believed applying emotional control skills in the classroom setting helped her gain confidence over time in her teaching. Overall, our findings suggest that successful life skills transfer involves mutually beneficial interactions between athletes and the contexts in which they apply the skills learned during sport participation. Ultimately, the findings extend the transfer literature by illustrating how the skills developed in sport are actually applied beyond sport.

\section{D2.P35. Psychological skills training for musicians}

\section{JOHANNES L. HATFIELD*}

\section{Norwegian Academy of Music \\ *Corresponding author: johannes.l.hatfield@nmh.no}

Guidance regarding psychological preparation for high performance is paramount for both musicians and athletes. However, there are surprisingly few research studies in music with the main objective of enhancing musicians' psychological skills. In music research, Self-Regulated Learning (SRL) has for more than two decades been applied as a conceptual framework for research on instrumental practice. However, this body of research has mainly been concerned with exploratory aspects such (e.g., to what degree music students are self-regulated, what self-regulation strategies music students apply during instrumental practice, and how one might facilitate self-regulated learning in children and adult music learners). With this in mind, there is a need for SRL intervention research providing musicians with psychological prerequisites applicable in an increasingly competitive work environment. The aim of the present research was to investigate personal benefits, perceptions, and the effect of a 15-week psychological skills training programme implemented within a frame of SRL. A mixed methods intervention study was selected as a suitable research design including effect size measures (i.e., T-test and Wilcoxon's signed rank) and a triangulation of qualitative methods (i.e., semi-structured interviews, a research log, and participants' diaries). Results revealed that the psychological skills intervention increased students' ability to accept adversities and take chances, this in turn, reduced participants' worry and anxiety in performance situations. The quantitative results revealed that the participants had increased in psychological skills such as goal setting ( $\mathrm{t}=4.90 ; P=<0.01)$, imagery $(\mathrm{t}=3.05 ; P=<0.05)$, arousal-regulation $(\mathrm{t}=2.76 ; P=$
$<0.05)$, concentration $(\mathrm{t}=4.74 ; P=<0.001)$, self-observation ( $\mathrm{t}=9.22 ; P=<0.0001)$, worry $(Z=-2.20 ; P=<0.05)$ and coping $(Z=2.33 ; P=<0.05)$. An 8-month follow up interview revealed that the participants were still actively applying the psychological skills. Combined, the present research reveals the effectiveness of both the implementation and application of principles from SRL and psychological skills in the field of music. In essence, the results yields that these two aspects should be further investigated in relation to one another. Moreover, the efficiency of SRL in music might be improved including psychological constructs addressing performance enhancement as part of the SRL model.

D2.P36. Making the team: an exploration of male student athletes' engagement in initiation activities

\section{MOIRA E. LAFFERTY ${ }^{1 *} \&$ CAROLINE WAKEFIELD $^{2}$}

\section{${ }^{1}$ University of Chester, ${ }^{2}$ Liverpool Hope University *Corresponding author: m.lafferty@chester.ac.uk @ProfMoiraL}

Despite a zero tolerance approach by the National Union of Students, British Universities and Colleges Sport, and higher education institutions initiation ceremonies still take place as a means of welcoming new members to sporting teams (Lafferty et al., 2016, International Journal of Sport and Exercise Psychology). With the majority of research focused on North American and Canadian sport relatively little is known about initiation activities in a United Kingdom context, or why athletes engage in such behaviours. Waldron and Kowalski (2009, Research Quarterly for Exercise and Sport, 80, 291-302) have suggested that engagement could be explained by over conformity to two elements of the sport ethic model (Hughes and Coakley, 1991, Sociology of Sport Journal, 8, 307-325), namely making sacrifices and striving for distinction. Therefore, the aim of the present research was to examine the nature of initiation activities in male university sport players and explore whether emergent themes mapped to the sport ethic model. Following institutional ethical approval information advertising the study was sent to sporting societies at several higher education institutions. Athletes who met the inclusion criteria of having participated in an initiation ceremony were invited to contact the research team. This random purposeful sampling approach (Patton, 2002, Qualitative evaluation and research methods (3rd ed.). California: Sage) resulted in a participant sample of 19 male athletes (mean age: $20.4 \pm 1.5$ years) representing a range of sports. After giving informed consent each athlete participated in a semi-structured interview lasting between 35-50 min. All interviews were recorded, transcribed verbatim, and analysed through a two phase procedure of data organization and interpretation following established thematic analysis guidelines (Braun \& Clarke, 2006, Qualitative Research in Psychology, 3, 77-101). Results were captured within two dimensions, the initiation and outcomes representing 6 and 2 themes respectively. Within these dimensions emerging themes of the group structure and hierarchy, shared experiences, coercion, initiation challenges, health risk behaviours through alcohol 
consumption, and feelings of being a team member mapped to the four areas of the sport ethic model in contrast to the work of Waldron and Kowalski (2009). These findings suggest that there are both similarities and differences in initiation activity engagement of UK student athletes compared to the United States. Furthermore, the highlighted differences in over conformity to the sport ethic model suggest that intervention development to deter participation in initiation activities should be context and culturally specific.

\section{D2.P37. Understanding mental toughness in Scottish rugby union: an interpretative phenomenological approach}

\section{STEPHEN LECKEY ${ }^{1 *}$, VICTORIA PENPRAZE ${ }^{1}$, ROSS WHITE $^{2} \&$ NIALL MACFARLANE ${ }^{1}$}

\footnotetext{
${ }^{1}$ The University of Glasgow, ${ }^{2}$ The University of Liverpool *Corresponding author: s.leckey.1@research.gla.ac.uk @Lecks_1
}

Professional rugby teams invest vast amounts of time and resource into the evaluation of the physical, technical and tactical sides of the game, in an attempt to gain a competitive advantage. The physiological demands of the game have been well documented (Cunniffe et al., 2009, The Journal of Strength \& Conditioning Research, 23, 1195-1203), however there is currently a lack of empirical literature regarding mental toughness in professional rugby union. Thus, the purpose of this study was to explore player's and coach's perceptions of mental toughness, within Scottish Rugby Union. We utilised a qualitative, cohort study design and purposeful sampling was employed to gather participants. With institutional ethics approval, 5 current professional players (Mean age $=$ $27.4, \mathrm{SD}=2.1$ years, mean professional playing experience $=$ $8.0, \mathrm{SD}=0.7$ years) and 3 current professional coaches (Mean age $=42.0, S D=9.2$ years, mean professional coaching experience $=20.0, S D=6.2$ years) participated. Players and coaches were interviewed individually, following a semi-structured interview schedule. Interview data was transcribed verbatim and an Interpretative Phenomenological Approach (IPA) was utilised to derive themes (Smith (Ed.), 2007, Qualitative psychology: A practical guide to research methods. Sage). The results identified key characteristics (goal-orientated, growth mentality, self-determined) and behaviours (performance behaviours, work ethic, sacrificial acts) associated with mentally tough rugby players. Analysis of this data also exposed the challenges and situations that demand mental toughness (return from injury, team (de)selection), along with the sociocultural influences (team values, cultural architects) on mental toughness development. We have extended the mental toughness literature by detailing the characteristics and behaviours of mentally tough rugby players. The practical implications of these findings are discussed, with a focus on the identification and development of mental toughness characteristics and behaviours within Scottish rugby union.
D2.P38. Individual and combined effects of externally focused instruction and enhanced performance expectancy on motor learning

DAVID MARCHANT*, LORCAN CRONIN, EMMA HUNTLEY, EVELYN CARNEGIE \& PAUL ELLISON

\author{
Edge Hill University \\ *Corresponding author: \\ david.marchant@edgehill.ac.uk
}

Enhanced expectancies for positive performance outcomes and an external focus of attention have independent and additive beneficial effects on motor learning. Expectancies are often enhanced through (false) positive social-comparative feedback or through "good" performance feedback. Changing task performance criterion such that "good" performances appear achievable also elevates expectancies, but the interaction with externally focused instruction has not been explored. The present study examined the independent and combined effects of externally focused instruction and performance criterion based enhanced expectancies on motor learning. The study was approved by university ethical review. In a $2 \mathrm{X}$ 2 design, fifty-three novice golfers (naïve to the study purpose) practicing putting golf balls (5 blocks of 10 putts to a target $150 \mathrm{~cm}$ away) were or were not provided with external focus instruction, and did or did not putt under conditions to enhance performance expectancies. This resulted in 4 groups: external focus (EF), enhanced expectancy (EE), external focus/enhanced expectancy (EF-EE) and control (C). EF instructions directed attention towards the target and movement of the club. For $\mathrm{EE}$, balls coming to rest within a larger circle surrounding the target constituted a "good" trial. Participants completed delayed retention and transfer (from180 cm) tests with instructions and/or circle not provided. Practice: no significant effect of condition on practice performance. Retention test: a significant main effect for $\operatorname{EF}\left(F(1,49)=13.66, P=.001, n p^{2}=.22\right)$, with retention accuracy enhanced with $E F$ present. No main effect for $\mathrm{EE}\left(\mathrm{F}=1.07, P=.31, \eta p^{2}=.02\right)$, or $\mathrm{EF} \mathrm{X} \mathrm{EE}$ interaction $\left(F=.24, P=.62, \eta p^{2}=.01\right)$ observed. Transfer test: a significant main effect for $E F\left(F=7.76, P=.01, n p^{2}\right.$ $=.14)$, with accuracy enhanced with EF. No EE main effect $\left(\mathrm{F}=.51, P=.48, n p^{2}=.01\right)$, or EF X EE interaction $(\mathrm{F}=.06$, $\left.P=.81, \eta p^{2}=.001\right)$ observed. Post-practice self-efficacy (SE) was enhanced in the presence of $E E$, but no differences were observed pre-retention. The findings support the proposal that effects of EF and EE mediate learning through different mechanisms. Overall, EF promoted enhanced learning, yet the learning of the EF-EE group was superior to the EE alone group. As such, whilst the EE criterion did not benefit SE and performance, the EF promoted performance and SE. Therefore, the effects of EF instruction were robust despite non-optimal motivation, supporting proposals that it has a greater influence on motor learning. 
D2.P39. We all look but do we see? Evaluating an observation intervention for trainee practitioners

\section{EMILY MARTIN ${ }^{1 *}$, STACY WINTER ${ }^{1} \&$ TIM HOLDER $^{2}$}

${ }^{1}$ St Mary's University, ${ }^{2}$ University of Central Lancashire

*Corresponding author: emily.martin@stmarys.ac.uk @emilymartin 11

The practice of observation has received limited literary attention within the sport psychology domain, despite its propensity to provide insightful information and direct assessment of client behaviour (Holder \& Winter, 2017, Sport, Exercise, and Performance Psychology, 6, 6-19). The current study aimed to deliver and evaluate an observation intervention to enhance the professional development of trainee practitioners. Specific objectives of the intervention were to encourage innovation of tangible ideas for immediate use in applied practice, and to develop comfort and confidence in implementing observation. All participants were registered on either the British Association of Sport and Exercise Sciences (BASES) supervised experience or British Psychological Society (BPS) stage-2 training pathways. Following institutional ethical approval, the final sample included 14 participants in the intervention group (mean age: $29.79 \pm 8.74$ years) and nine participants in the control group (mean age: $28.89 \pm 4.01$ years). The one-day intervention workshop comprised of three main activities (symposia, practical application, and role plays) aimed to encourage active participation in sharing, applying, and developing knowledge of observation (Tashman \& Tenenbaum, 2013, Journal of Sport Psychology in Action, 4, 71-85). The control group did not receive any formal observation training. Six indicators (value, comfort, confidence, competence, understanding, and frequency of observation) were used to measure intervention effectiveness across three time points (pre-intervention, two-weeks post, and six-months post intervention) to determine the maintenance of any change to observation practice. Results demonstrated the intervention had been effective in prompting a significant increase $(P<.001)$ in trainee observation practices from pre to six-months post intervention, for confidence, comfort, and understanding, when compared to the control group $(P>.05)$. Follow-up qualitative enquiry was also conducted six-months post intervention to extend quantitative findings. Using an interpretative phenomenological analysis (IPA) approach, semi-structured interviews were used to collect data from seven participants selected from the intervention group via maximum variation sampling (Sparkes \& Smith, 2014, Qualitative research methods in sport, exercise and health: From process to product. Oxon: Routledge). Four superordinate themes emerged: situational variables that influence observation frequency, changes made to observation approach, influential learning moments, and the perceived effectiveness of the intervention. Overall, findings support the intervention effectiveness in eliciting and maintaining change in trainee practitioners' observation practices. As such, the current gap in observation research requires further development, particularly surrounding the training of its use among applied practitioners.
D2.P40. Preliminary validation of the Exercise Attentional Questionnaire in physical education

MAXIME MASTAGLI*, JEAN-PHILIPPE HAINAUT, AURÉLIE VAN HOYE \& BENOIT BOLMONT

\author{
Université de Lorraine \\ *Corresponding author: \\ maxime.mastagli@univ-lorraine.fr
}

Emotional and attentional states have received considerable attention in exercise psychology. However, very few questionnaires have been developed to measure attentional states within the physical education context. The purpose of this paper was to develop and validate the "Exercise Attentionnal Questionnaire (EAQ)" in a French student sample. After an item selection using a committee approach, study 1 underlines the content validity of a pool of 20 items $(N=20)$. Study 2 tested the factorial structure of the questionnaire using exploratory and confirmatory factor analysis among Faculty of Sport Sciences students $(N=164)$, as well as external validity with selfconfidence and anxiety relationship (Martens, Burton, Vealey, Bump, \& Smith, 1990, Competitive anxiety in sport (pp. 127-140). The supported model is composed of four factors and indicates satisfactory internal consistency: concentration on the task ( $\alpha=.88 ; 5$ items; e.g., I was concentrated to succeed), distraction on the task (a $=.78$; 4 items; e.g., I happened to think of something else rather than exercise), concentration through teacher support ( $\alpha=.88 ; 5$ items; e.g., I felt that the teacher was helping me to concentrate on the exercise), and distraction through teacher support ( $a=.79 ; 4$ items; e.g., During the exercise, I was unconcentrated by the teacher). Low but significant correlations were found between "self-confidence" and "concentration on the task" ( $r=-.275 ; P<.001)$, and also with "concentration through teacher support" $(r=-.188 ; P<.05)$ and between "cognitive anxiety" and "distraction on the task" " ( $r=.159 ; P<.05)$. One-way ANOVA, with the four factors of the questionnaire as dependent variable, found no differences between boys and girls $(P>.05)$. Nevertheless, significant differences $(P<.05)$ found due to social desirability (Reynolds, 1982, Journal of clinical psychology, 38(1), 119-125 ("higher social desirable" have higher "concentration on the task" and "concentration through teacher support")), and to the type of sport practiced outside of university ("no practice" have higher "concentration on the task" than "individual sport") and depending on the teacher giving the lesson for "concentration on the task", "concentration through teacher support" and "distraction on the task" $(\mathrm{N}=6)$. Present findings provide preliminary evidence for the validity of the EAQ. The present work is in progress to increase the sample and examine the structure validity among other samples and others exercises contexts. 
D2.P41. The contribution of coping related variables and cardiac vagal activity on prone rifle shooting performance under pressure

\section{EMMA MOSLEY ${ }^{1,2^{*}}$, SYLVAIN LABORDE ${ }^{3,4}$ \& EMMA KAVANAGH ${ }^{2}$}

\author{
${ }^{1}$ Southampton Solent University, ${ }^{2}$ Bournemouth \\ University, ${ }^{3}$ German Sport University, ${ }^{4}$ University of Caen \\ *Corresponding author: emma.mosley@solent.ac.uk \\ @emma_mosley
}

Understanding athletic performance under pressure is vital. Recently a combined approach to this area has been examined including several facets of psychophysiology such as personality traits, stress appraisals and cardiac vagal tone, inferred from heart rate variability measurement (Laborde, Lautenbach, \& Allen, 2014, Journal of Physiology and Behaviour, 139, 532-540). It has been suggested that a combination of different factors allows for better prediction of athletes performance under pressure. Thus the aims of this study were (1) to assess the predictive role of coping related variables (CRV) on cardiac vagal activity (derived from heart rate variability), and (2) to investigate the influence of CRV (including cardiac vagal activity) on prone rifle shooting performance under low pressure (LP) and high pressure (HP) conditions. After institutional ethical approval, participants $(n=38$, mean age $=55 \pm 14.8$, years experience $=31.1 \pm$ 21.5) competed in a shooting task under LP and HP conditions. Cardiac vagal activity measurements were taken at baseline, task and during recovery for $5 \mathrm{~min}$. Self-reported ratings of stress were recorded at three time points via a visual analogue scale. Upon conclusion of the task, self-report measures of motivation, stress appraisal, attention, perceived pressure and trait CRV questionnaires (Decision Specific Reinvestment Scale, Movement Specific Reinvestment Scale and Trait Emotional Intelligence Questionnaire) were completed. Results indicated that resting cardiac vagal activity had no predictors. Task cardiac vagal activity and cardiac vagal reactivity (difference from resting to task) was predicted by resting cardiac vagal activity and trait El self control in both LP and HP conditions. Cardiac vagal recovery (difference from task to post task) was predicted by decision reinvestment in HP $\left(R^{2}=.08, P=.045\right)$. Shooting task performance was predicted by experience and post task cardiac vagal activity in the LP condition which accounted for $22 \%$ of the variance in shooting score. In the HP condition cardiac vagal recovery and trait El emotionality accounted for $28 \%$ of the variance in shooting score. The current research suggests that a combination of CRV including cardiac vagal activity variables influence cardiac vagal activity throughout a pressure task. It also indicates that shooting performance is positively influenced by effective cardiac vagal recovery.

\section{D2.P42. Examining the association between mental toughness and metacognition}

\author{
JOHN K. PARKER ${ }^{1 *}$, MARTIN I. JONES $^{2} \&$ GEOFF P. \\ LOVELL $^{3}$ \\ ${ }^{1}$ University of Gloucestershire, ${ }^{2}$ University of Exeter, \\ ${ }^{3}$ University of the Sunshine Coast \\ *Corresponding author: jparker@glos.ac.uk
}

Metacognition refers to our ability to control, modify, and interpret cognitive activity (Wells \& Cartwright-Hatton, 2004, Behaviour Research and Therapy, 42, 385-396). Research suggests dysfunctional metacognitive beliefs can be an important factor in the development and maintenance of mental health problems (Cotter, Yung, Carney, \& Drake, 2017, Behaviour Research and Therapy, 90, 25-31). Mental toughness represents a collection of personal resources that may act as a positive indicator of mental health (Gucciardi et al., 2016, Journal of Science and Medicine in Sport, 20, 307-311). Therefore the purpose of our study was to assess whether an inverse relationship existed between mental toughness and metacognitive factors associated with psychopathology. Following institutional ethical approval 60 undergraduate university students (mean age: $21.07 \pm 4.19$ years) studying various sport science related degree programmes voluntarily consented to participate in the current study. Participants completed the Metacognitions Questionnaire-30 (Wells \& Cartwright-Hatton, 2004, Behaviour Research and Therapy, 42, 385-396) and Mental Toughness Index (Gucciardi et al., 2015, Journal of Personality, 83, 26-44). Questionnaires were counterbalanced to reduce order effects with participants on average taking nine minutes to complete both questionnaires. A Pearson bivariate correlation recorded significant negative relationships between the mental toughness index, total MCQ-30 ( $r=-.334, P<0.01$, BCa 95\% Cl: $-.639-.084)$ scores, positive beliefs about worry $(r=-.329, P=<0.01$, BCa 95\% Cl: $-.652-.010)$, and negative beliefs about thoughts concerning uncontrollability and danger $(r=$ $-.330, P=<0.05$, BCa $95 \% \mathrm{Cl}:-.652-.109)$ subscale scores. Our investigation suggests that mental toughness is a statelike psychological resource that may contribute to people being less likely to adopt metacognitive beliefs, judgements, and monitoring tendencies associated with the development and maintenance of psychological disorders. More specifically, mental toughness scores were negatively associated with positive beliefs about worry suggesting that participants were unlikely to consider worrying as a means to cope and avoid problems in the future. In addition, responses to items indicative of persistent worrying thoughts that can be accompanied with unpleasant somatic effects were also inversely associated with mental toughness scores. Practitioners may wish to consider developing higher levels of mental toughness as a means of mitigating against the adoption of negative metacognitive processing and in doing so minimise the emergence of negative mental health symptoms (e.g., depression, anxiety).

\section{D2.P43. Identification of central context-sensitive aspects of trust in volleyball teams via a mixed methods approach}

\section{CHRISTINA PLATH*}

\section{University of Vechta}

*Corresponding author: christina.plath@uni-vechta.de

Trust plays an important role in the context of team sports, within relationships between team members, and regarding to performance (Mach et al., 2010, Journal of Occupational and Organizational Psychology, 83, 771-794). Besides, trust 
works as a complexity-reducing mechanism by which certain options of operations even become possible (Luhmann, 2014, Vertrauen. Ein Mechanismus der Reduktion sozialer Komplexität. Konstanz: UVK). A differentiated analysis of personal and situational factors of trust especially for the interactive sports' context is still missing up to now. Therefore, the aim of this survey was to identify the systematic differences of the subjective perception concerning the relevant components, antecedents and effects of trust within volleyball teams. Therefore a mixed methods approach with two phases (first qualitative then quantitative inquiry) was chosen. With institutional ethics approval, 'phase 1 ' included the conduct and analysis of 12 structured interviews ( $N=12)$ according to the method of empirical based construction of types (Kelle \& Kluge, 2010, Vom Einzelfall zum Typus. Fallvergleich und Fallkontrastierung in der qualitativen Sozialforschung. Wiesbaden: VS). Four qualitatively different types of trust were identified (task-focused type, task- andsocial-focused type, undifferentiated type, hierarchical-controlling type) which differ concerning the subjective perception of central antecedents, effects as well as the general relevance of trust in the context of volleyball. On the basis of the qualitative results a questionnaire was developed in 'phase 2 ' which measures the subjective perception of antecedents, situational factors as well as effects of available trust within volleyball teams. The questionnaire was used at three different times. A five-item scale of "context-sensitive effects of trust" was estimated (t1: $a=0.752 ; \mathrm{t} 2: a=$ 0.766 ; $\mathrm{t} 3: a=0.743)$. A cluster analysis based on this scale identified three different clusters at time $1\left(X^{2}(2, N=285)=\right.$ 217.311, $P<0.01)$ and $2\left(X^{2}(2, N=302)=231.567, P<0.01\right)$ and two different clusters at time $3(U=45.00 ; P<0.01)$. The findings of the survey stress the importance of considering the context-specific elements as well as individual differences of relevant trust components for successful individual performance and team interactions.

\section{D2.P44. Three images not to worry about Worry}

YAGO RAMIS $^{1 *}$, CARME VILADRICH ${ }^{1}$, MIQUEL TORREGROSSA ${ }^{1}$, ALEXANDER LATINJAK ${ }^{2,3}$ \& JOAN PONS ${ }^{1}$

\section{${ }^{1}$ Universitat Autònoma de Barcelona, ${ }^{2}$ University of Suffolk, ${ }^{3}$ School for Sport and Health Sciences (EUSES), University of Girona \\ ${ }^{*}$ Corresponding author: yago.ramis@uab.cat @YagoRamisUAB}

Competitive anxiety is defined as a sport-specific affective response that appears before or during competition (e.g., Martens, 1977, Sport Competition Anxiety Test). Where state anxiety refers to the situational affective overactivation before a specific competition, trait competitive anxiety is the tendency of individuals to react to competitive scenarios with state anxiety. Measures developed to assess both trait and state competitive anxiety have included both somatic and cognitive factors in their inventories and, although different instruments vary on the assessment of physical and cognitive symptoms, one factor common to most measures and which seems to be generally associated with cognitive anxiety is the factor labelled as Worry. Most research that has assessed competitive anxiety using questionnaires tends to find that both elite and young athletes report systematically higher levels of Worry than other anxiety factors, frequently suggesting that athletes worry too much. During the last decade, we have focused specifically on assessing young athletes' competitive anxiety in multiple studies, all of them ethically approved by a higher education institution. We will present three graphical results to discuss the relationship between the Worry factor and other anxiety subscales (i.e., somatic anxiety, concentration disruption and self-confidence) as well as with the general competitive anxiety levels: (a) Confirmatory factor analysis of the SAS-2 will present information about the stability of the structure of the questionnaire along studies and populations and will show the similarities of this factor in comparison with the factors of somatic anxiety and concentration disruption; (b) The information function of the three subscales of the SAS-2 as well as of their 15 items, will show how each factor of the SAS-2 informs about different aspects of general competitive anxiety and how athletes report levels of worry at low levels of competitive anxiety whereas somatic anxiety and concentration disruption only arise with higher levels of general competitive anxiety; (c) A network analysis based on Qgraph package for R (Epskamp, Cramer, Waldorp, Schmittmann, \& Borsboom, 2012, Journal of Statistical Software, 48, 1-18), will inform of the differentiated relationships of the factors of the SAS-2 with variables such as appraisals, competitive emotions and self-confidence. We will finally discuss on the interpretation of the reported levels of worry in athletes to explain that this anxiety factor should not be considered essentially as a negative consequence in youth sports, but as an indicator of athletes' adequate appraisal of the importance of the competition at stake.

\section{D2.P45. Psychological problems in youth sports}

\section{LIUDMILA ROGALEVA* \& VALERII MALKIN}

\section{Ural Federal University \\ *Corresponding author: I.n.rogaleva@urfu.ru}

The main problem in the youth sports is a small percentage of young talented sportsmen who achieve top results in adult sports. One of the reasons of this situation is related to the peculiarities of young athlete' personality. The purpose of the study is to reveal specific change in personality development in young athletes. More than 140 sportsmen of 15-17 years took part in the study. After two years of training at the Olympic Reserve School we defined young sportsmen were showing poor progress. Analysis of the psychological indicators of the sportsmen's data has shown that after 2 years of training these sportsmen demonstrated the increase in material motives for doing sports - from 17 to $39 \% P>0.001$, the prestige values - from 37 to $41 \%$, and the decrease in the self-improvement motives from 23 to $12 \% P>0.05$. The self-esteem studies showed the increase in the number of sportsmen with low self-esteem from 23 to $36 \% P>0.05$, the decrease in the number of sportsmen with reasonable self-esteem from 34 to $26 \% P>0.05$. At the same time we studied the psychological climate within the groups of these sportsmen (Khanin, 1980). We indicated the increase in such indicators as indifference from 3.6 to $6.1 P>0.001$, 
disappointment with sports activities from 4.2 to $6.3 P>$ 0.001 , lack of cooperation from 4.1 to $5.6 P>0.001$. Studies of the dynamics of the relations with a coach demonstrated the decrease in the assessment of professionalism from 5.4 to $3.3 P>0.001$, the component of emotional relations from 4.4 to $2.6 P>0.001$, the behavioral component from 5.6 to 3.2 scores $P>0.001$. The sports results of these sportsmen were unstable; they tended to show worse performance. More than $60 \%$ of the young sportsmen finished their sports activities in a few years. The conducted studies show that in the course of the sports activities young sportsmen often demonstrate the decline of a number of personal characteristics and psychological indicators. This leads to significant psychological problems, and does not contribute to forming a psychologically stable and self-confident personality. These data allow us to assume that one of the reasons for the fact that many young sportsmen do not achieve top results in the adult sports is the underestimation of the importance of psychological work with the young sportsmen by the coaches.

\section{D2.P46. The impact of stress on empathic accuracy in coach-athlete dyads participating in elite level individual based sports \\ ELIZABETH SCHOLEFIELD ${ }^{1 *}$, STEPHEN PACK ${ }^{1}$ \& ROSS LORIMER $^{2}$ \\ ${ }^{1}$ University of Hertfordshire, ${ }^{2}$ Abertay University \\ *Corresponding author: esscholefie1@yahoo.co.uk @ESSPsych}

Characterised by a demand to perform to optimum levels in intense pressure situations, together with the highly visible and public nature of performance outcomes, elite sport has the potential to be extremely stressful. At times of stress both coaches and athletes have reported difficulties in maintaining positive and effective interactions within their coach-athlete partnerships (Holt \& Hogg, 2002, The Sport Psychologist, 16, 251-271; Olusoga et al., 2009, Journal of Applied Sports Psychology, 4, 442-459). Accurate interpersonal perception is a key skill in maintaining positive interactions, therefore the present study explored the impact of stress on levels of empathic accuracy achieved by coach-athlete dyads operating in elite sport. With institutional ethics approval and in line with previous investigations exploring empathic accuracy, 4 elite coaches (mean age: $36.6 \pm 4.8$ years) and 20 elite athletes (mean age: 18.5 \pm 1.7 years), forming 20 coach-athlete dyads, volunteered to participate from a range of individual based sports (e.g. gymnastics, cycling, athletics and swimming). An adaptation of the unstructured dyadic interaction paradigm (Lorimer and Jowett, 2009, Psychology of Sport and Exercise, 10, 152-158) was used to explore empathic accuracy, whereby each dyad was filmed during two training sessions and one competition. Dyad members individually viewed selected video footage of interactions that had occurred during each recorded session, recalling what they remembered thinking/feeling during the interactions, while making inferences about what their partner's thought/felt at each point. Comparisons of participant's self-reports and inferences for each interaction were used to calculate a percentage score of empathic accuracy during each session. Participants completed a simple stressor frequency scale to establish levels of stress experienced in training compared to competition. It was found that empathic accuracy achieved by elite coaches and athletes remained relatively stable across both training sessions (coaches training 1: $M=31.4, S D=11.87$; coaches training 2: $M=31.79, S D=7.87$; athletes training $1: M=25.48, S D=$ 11.06; athletes training $2: M=26.50, S D=15.95$ ), however accuracy was seen to increase during competition (coaches competition: $M=42.49, S D=18.27$; athletes competition: $M$ $=35.85, S D=17.08)$. In addition, all participants reported experiencing significantly increased stress during competition, compared to training $(Z=5.19, P<.001)$. The results suggest that the distinct nature of the elite training and competition environments can affect levels of empathic accuracy achieved within coach-athlete dyads operating in individual based sports. This has implications for understanding how coach-athlete interactions are influenced by stress.

D2.P47. The role of decision specific reinvestment in pattern recall in rugby union

\section{SEBASTIAN SHERWOOD ${ }^{1,2^{*}}$, TIAKI SMITH ${ }^{1,2} \& \mathrm{RICH}$ MASTERS $^{1,3}$

\author{
${ }^{1}$ University of Waikato, ${ }^{2}$ Chiefs Super Rugby team, \\ ${ }^{3}$ University of Hong Kong \\ *Corresponding author: seb_49_5@hotmail.co.uk
}

The Decision Specific Reinvestment Scale (DSRS) can be used to assess an individual's predisposition for conscious processing of decisions. The Scale assesses decision reinvestment (an individual's propensity to consciously monitor the processes involved in making a decision), and decision rumination (an individual's propensity to focus on negative evaluation of previous poor decisions). Evidence suggests that individuals who score highly on the DSRS perform worse, and make slower decisions, under pressure in sports such as basketball (Kinrade et al., 2015, Psychology of Sport and Exercise, 20, 11-19), netball (Jackson et al., 2013, International Journal of Sport Psychology, 44, 331-350) and korfball (Kinrade et al., 2010, Journal of Sport Sciences, 28, 1127-1135). To date, the propensity for decision specific reinvestment has not been investigated in rugby union. We used the DSRS to examine the influence of consciousness on decision-making during a pattern recall task. Pattern recall is an essential skill for a rugby union player, it facilitates decision-making and has been shown to explain up to $40 \%$ of the variance on an anticipation task (Farrow et al., 2010). To gain an understanding of the role of decision reinvestment and rumination in pattern recall ability in elite rugby players. Fifty-seven elite rugby union players (age $22.63 \pm 3.14$ years) viewed still images of structured rugby patterns $(\mathrm{N}=20)$ for 5 s before occlusion. Participants were instructed to recall player positions by marking them on a blank template as quickly and accurately as possible. Players' recall accuracy $(\mathrm{cm})$ and response time (s) were measured and decision reinvestment and rumination were assessed using the DSRS. Ethical approval was obtained and all participants signed informed consent. 
Pearson correlations revealed a significant negative correlation between decision reinvestment and pattern recall accuracy $(r=-.348, P=0.008)$. Decision reinvestment scores were also positively associated with response time $(r=.359$, $P=0.006)$. Rumination was not significantly associated with recall accuracy or decision time. The results suggest that players with a higher propensity to consciously monitor their decision-making took longer to make decisions but, paradoxically, were less accurate at recalling patterns, compared to players with a lower propensity to consciously monitor their decision-making. Sperling (1960, Psychological monographs: General and applied, 74, 1) showed that iconic memory for visual information is substantial but decays extremely rapidly, so increased time between encoding and retrieval of visual information disrupts recall. High reinvestors in our study may have demonstrated poorer recall for associated reasons.

D2.P48. How do goals influence subjective experience during performance? An experimental test of the effects of goal types on performance, flow and clutch states, confidence and perceived challenge

\section{CHRISTIAN SWANN*, MATTHEW SCHWEICKLE, SCOTT GROVES \& STEWART A. VELLA}

\section{University of Wollongong \\ *Corresponding author: cswann@uow.edu.au @cswannpsych}

Optimal subjective experiences are highly desirable for practitioners, coaches, researchers, and individuals engaging in sport and exercise. Recent qualitative evidence suggests that two such experiences (flow and clutch) underlie excellent performances in sport (Swann et al., 2017, Journal of Applied Sport Psychology). Furthermore, this research suggests that the type of goal pursued determines which state is experienced, and that such goals are causal mechanisms for the occurrence of each state. To build on that qualitative work, this study aimed to examine the effects of goal types on flow and clutch states during task performance. Secondary aims were to investigate the effects of goal types on objective performance, subjective perceptions of performance, confidence, and perceived challenge. Participants $\left(N=95 ; M_{\text {age }}=24.89, S D=9.27\right.$ ) were randomised to one of three goal conditions: (i) open goals (e.g., "see how well you can do"); (ii) specific goals of incrementally increasing difficulty; and (iii) do-your-best goals. Participants were asked to perform a Letter and Number Identification Task (Hardy \& Fazey, 1990, Mental training, Leeds: National Coaching Foundation) repeated six times, which provided a measure of objective performance. Flow and clutch states were measured on a dichotomous scale including six items. Perceptions of performance, confidence, and perceived challenge were each measured using single-items. Dependent variables were analysed separately employing a repeated measures design (mixed model). Results indicated significant interactions between goal types and all dependent variables. Participants prescribed open or do-your-best goals experienced significantly higher levels of flow than those prescribed specific goals; who conversely experienced significantly higher levels of clutch states. Participants assigned specific goals performed significantly better than those prescribed open or do-your-best goals. However, those assigned open or do-your-best-goals reported greater subjective perceptions of performance, higher confidence, and feeling more optimally challenged. These findings provide experimental support for the role of goal types in determining flow and clutch states, and further understanding of the psychological effects of these goal types. Specifically, these findings suggest that specific, challenging goals - widely advocated as best practice (Locke \& Latham, 2002, American Psychologist, 57, 705-717) - induce experiences of lower confidence, higher challenge, and lower perceptions of performance. Conversely, open and do-your-best goals induce experiences of higher confidence, more optimal challenge, and higher perceptions of performance. Implications of these findings are discussed in terms of application to research and applied practice in sport and exercise, including contexts such as exercise prescription and promotion.

\section{D2.P49. Career trajectories: the not always easy path to dual career}

\section{MIQUEL TORREGROSSA ${ }^{1 *}$, SUSANA PALLARES ${ }^{1}$, JOSE L. CHAMORRO $^{2}$, YAGO RAMIS ${ }^{1}$, ALEXANDER LATINJAK ${ }^{3}$ \& ANNA JORDANA ${ }^{1}$}

\section{${ }^{1}$ Universitat Autonoma de Barcelona, ${ }^{2}$ Universidad Europea de Madrid, ${ }^{3}$ Escola Universitaria de Salut i Esport \\ *Corresponding author: miquel.torregrossa@uab.cat @miktorregrossa}

A dual career (DC) is defined as a career with the major foci on sport and studies or work (Stambulova \& Wylleman, 2015, Psychology of Sport and Exercise, 21, 1-3). Its promotion is a priority both to prevent problems following retirement from elite sport and to provide opportunities for those talented athletes that will not make a successful junior to senior transition. Before the definition and use of DC term, we proposed three different models of trajectories depending on how the athletic and the educational-vocational level were combined (Pallarès, Torregrossa, Azócar, Selva, \& Ramis, 2011, Cultura, Ciencia y Deporte, 17, 93-103). Athletes follow 3 different trajectories: (a) linear when they are exclusively focused on athletic career, (b) convergent when they prioritize sport over education or employment, and (c) parallel when both dedications are equally prioritized. However, following one type of trajectory or another is not always the athletes' choice and depends on the sporting culture of each sport. With institutional ethics approval, four different studies ( $n_{1}=20$ water polo players, $n_{2}=23$ individual sports athletes, $n_{3}=6$ basketball players, $n_{4}=16$ soccer players) were conducted to describe the trajectories followed by athletes in different sports. Results showed three different groups of sports regarding trajectories and DC. In 'super-professionalized sports' (i.e., soccer) athletes find significant barriers to follow parallel or convergent trajectories and are often forced by the environment (clubs, managers, coaches) to follow linear trajectories if they want to pursue a professional sporting career. In 'semi-professionalized sports' (e.g., water polo) the lack of 
economic resources and big contracts compels athletes to follow either convergent (sport combined with a subordinated job in the same club) or parallel careers (sport combined with studies). In 'traditional Olympic sports', most of them non-professional (e.g., track and field, rowing), athletes find more flexibility and possibilities to choose their trajectories, varying their priorities as a function of Olympic cycles. Following parallel and convergent trajectories (DC) should be an athletes' choice not determined only by the group of sports and their sporting culture. Considering that following parallel and convergent trajectories (DC) have shown long term benefits for retirement (Torregrossa et al., 2015, Psychology of Sport and Exercise, 21, 50-56) its promotion is more urgent in 'super-professionalized sports' were a cultural change at an organizational (clubs and federations) and personal level (coaches and families) is needed.

\section{D2.P50. The effects of challenge and threat states on competitive sprint cycling performance}

\section{NATHAN WOOD ${ }^{1 *}$, LEE J. MOORE ${ }^{4}$, JOHN PARKER ${ }^{1}$, MATTHEW I. BLACK ${ }^{3}$ \& PAUL FREEMAN ${ }^{2}$ \\ ${ }^{1}$ University of Gloucestershire, ${ }^{2}$ University of Essex, \\ ${ }^{3}$ Loughborough University, ${ }^{4}$ University of Bath \\ *Corresponding author: Nathanw53@gmail.com @nathanMwood}

Studies have shown that while a challenge state aids sports performance, a threat state hampers performance (Moore et al., 2012, Psychophysiology, 49, 1417-1425). The cardiovascular pattern exhibited during a challenge state (relatively higher cardiac activity and lower resistance in the blood vessels), is thought to provide an efficient spike of energy to the brain and muscles that prepares the body for immediate action. This short burst of energy has been predicted to enhance the performance of tasks requiring anaerobic power (Jones, et al., 2009, International Review of Sport and Exercise Psychology, 2, 161-180). However, to date, no research has examined this proposition. Thus, this study aimed to assess the effects of challenge and threat states on competitive sprint cycling performance. With institutional ethical approval, 35 participants (22 male; mean age: $25.8 \pm 7.9$ years) completed familiarisation, baseline, and pressure sprints on a cycle ergometer (Lode Excalibur Sport V2, Groningen, Netherlands). The 30-s sprints were preceded by a 3-min warm-up and separated by 10-mins of rest. Before the pressure sprint, participants received instructions about the competitive nature of the sprint (e.g., prizes and punishments for the best and worst performing participants, respectively). Challenge and threat states were then assessed via self-report (demand resource evaluation score [DRES]) and cardiovascular reactivity (challenge threat index [CTI]; as Moore et al., 2013, Journal of Sport and Exercise Psychology, 35, 551-562). Finally, sprint performance was assessed in terms of change in peak power (PP) from the baseline to pressure sprint. The results revealed that while participants exhibited an increase in heart rate before the pressure sprint $(2.23 \pm 4.75 \mathrm{bpm} ; t(30)=2.62, P=.014)$, suggesting that they were actively engaged in the task, neither DRES $\left(R^{2}=.03, \beta=\right.$ $.18, P=.331,95 \% \mathrm{Cl}=-17.80$ to 51.12 , nor $\mathrm{CTI}\left(\mathrm{R}^{2}=.03, \beta=\right.$ $.17, P=.360,95 \% \mathrm{Cl}=-39.88$ to 14.93$)$, predicted change in PP. Thus, the results raise questions about whether challenge and threat states influence the performance of competitive sporting tasks that require anaerobic power, and suggest that further research is needed.

D2.P51. Associations between physical fitness and academic performance is independent of achievement motivation in Japanese junior high school students

\section{KOJ YAMATSU ${ }^{1 *}$, TOSHIHIRO NAKAJIMA ${ }^{2}$, NORITERU MORITA $^{3}$, TORU ISHIHARA ${ }^{4}$ \& MASATO SAGAWA ${ }^{3}$ \\ ${ }^{1}$ Saga University, ${ }^{2}$ Fuji Women's University, ${ }^{3}$ Hokkaido University of Education, ${ }^{4}$ Hokkaido University \\ ${ }^{*}$ Corresponding author: kyamatsu@cc.saga-u.ac.jp}

Recent research suggested that physical fitness was associated with academic performance in children and adolescents (Hillman, Erickson, \& Kramer, 2008, Nature Reviews Neuroscience, 9, 58-65). Achievement motivation may be related to academic performance (Uhlinger \& Stephens, 1960, Journal of Educational Psychology, 51, 259-266) and may be associated with physical fitness (Nishida \& Sawa, 1993, The Japanese Journal of Educational Psychology, 41, 125-134). Therefore, it is unknown whether achievement motivation moderates the relationship between physical fitness and academic performance. The purpose of this study was to examine the relationship between physical fitness and academic performance independent of achievement motivation. Participants were 394 (girls: 49.5\%, mean body mass index [BMI]: $19.0 \pm 3.0$ ) first year (7th grade in USA) students of public junior high schools in Japan. After obtaining institutional ethics approval, all students and their parents completed the questionnaires. Physical fitness scores and details of academic performance (school grade) were obtained from school records. The physical fitness tests (50$\mathrm{m}$ sprint, standing broad jump, repeated side-steps, sit and reach, sit-ups, hand grip strength, handball throw, and 20mshuttle run) that were authorized by the Japanese Ministry of Education, Culture, Sports, Science and Technology were used. Achievement motivation (self-fulfillment achievement motivation [SFAM] and competitive achievement motivation [CAM]) was evaluated through the Japanese version of achievement motivation questionnaires (Horino \& Mori, 1991, The Japanese Journal of Educational Psychology, 39, 308-315). The total grade points of the 8 school subjects (GP8; Japanese, Social studies, Math, Science, English, Music, Arts, and Home economics/Vocational technology) were used as academic performance scores. Physical fitness score was significantly correlated with GP8 $(r=0.275, P<0.05)$. SFAM was significantly correlated with GP8 $(r=0.142, P<0.05)$, but CAM was not significantly correlated with GP8 $(r=0.041$, n.s.). Multiple regression analysis after controlling for several covariates (gender, household income, fathers' and mothers' educational histories, BMI) showed that physical fitness score $\left(R^{2}=0.215\right.$, beta $\left.=0.255, P<0.05\right)$ was significantly related to GP8. However, SFAM (beta $=0.059$, n.s.) was not remain a factor for GP8. These results suggest that higher physical fitness was associated with better academic performance independent of achievement motivation among Japanese junior high school students. 


\section{Day 2. Posters - Sport and Performance}

\section{D2.P52. Drive performance indicators for able- bodied and disabled golfers}

\author{
IAN KENNY ${ }^{1 *}$, MARK CAMPBELL ${ }^{1}$, SEAN SURMON ${ }^{2} \&$ LIZ \\ BRESSAN $^{2}$ \\ ${ }^{1}$ University of Limerick, ${ }^{2}$ Stellenbosch University \\ *Corresponding author: ian.kenny@ul.ie
}

Golf is a popular leisure and competitive activity for individuals with disabilities (Bragaru et al., 2011, Sports Medicine, 41, 721-740). The current golf handicap system does not fully take into account challenges of playing golf with any form of physical disability. The aim of this study was to examine golf driving performance measures, comparing golfers with various types of physical disabilities to able-bodied golfers. Through drive shot ball launch analysis using a radar tracking system (EDH Flightscope X2), this study compared amputees (single leg), deaf, visually impaired, polio, Les Autres and arthrogryposis golfers to able-bodied golfers with similar golf handicaps. With institutional ethics approval, twentyseven able-bodied participants (handicap category 3 , $12.4 \pm 7.0$ ) and fifteen disabled participants (handicap category 3, $18.2 \pm 9.2$ ) hit ten drives each. Able-bodied golfers presented longer but less accurate drives (208.1 $\mathrm{m}$ carry, $22.8 \mathrm{~m}$ lateral deviation) than golfers with a disability $(157.6 \mathrm{~m}$ carry $[P<0.001, \mathrm{~d}=1.33, \mathrm{Cl}=185.9$ -194.2], $17.0 \mathrm{~m}$ lateral deviation $[P<0.01, \mathrm{~d}=0.07, \mathrm{Cl}=$ -7.1-3.1]). Shorter drive length indicates that the golfers with a disability might not be able to generate the body angular speeds equivalent to those of able-bodied golfers with the same golf handicap. Consequently golfers with a disability have to possess more accuracy in order to be competitive. Golfers with a disability hit more shots in regulation (on the fairway) (80.3\%) compared to their able-bodied counterparts (59.9\%). Ball sidespin $(P<0.05$, $\mathrm{d}=-0.51, \mathrm{Cl}-90.4-3.8)$ and backspin $(P<0.05, \mathrm{~d}=0.08$, $\mathrm{Cl}=2961.0-3139.7)$ components were lower for golfers with a disability, showing more control. Lower ball sidespin and side angle component magnitude for golfers with a disability demonstrated that their more accurate drive performance was not as a result of shorter drives. The difference in outcome performance cannot be accounted for by the small difference in golf handicap score, thus golfers with a disability appear to be penalised/ disadvantaged by the current golf handicap classification rules. Implicit is the concern that a golf handicap calculated based on scores earned on one type of course (e.g. relatively flat course, few bunkers) might not accurately reflect the potential for a golfer with a disability on a very different type of course (e.g. very hilly course). Golfers with a disability developed effective ball launch compensatory strategies to overcome the challenges presented by playing golf with their respective disabilities.
D2.P53. Next day subjective and objective recovery indices following acute low and high training loads in team sport players

\section{MARK R. NOON ${ }^{1}$, ROB S. JAMES ${ }^{1}$, NEIL D. CLARKE ${ }^{1}$, RICHARD J. TAYLOR ${ }^{2} \&$ C. DOUGLAS THAKE ${ }^{1}$ \\ ${ }^{1}$ Coventry University, ${ }^{2}$ Newman University \\ *Corresponding author: aa5349@coventry.ac.uk @mark_mrnoon}

Assessments applied to monitor aspects of athlete recovery must be sensitive to changes in recovery status induced by different training loads. The aim of this study was to determine the sensitivity of selected subjective and objective monitoring assessments in detecting changes in group and individual responses to low and high load bouts of high intensity intermittent exercise. In a counterbalanced crossover design, ten Academy Rugby Union players (mean \pm SD: age: $18 \pm 1$ years, stature: $180 \pm 7 \mathrm{~cm}$, body mass: $86.6 \pm 18.5$ $\mathrm{kg}$ ) performed a low load (15 $\mathrm{min}$ ) and a high load (90 min) bout of high intensity intermittent exercise (Loughborough Intermittent Shuttle Test) one week apart. A seven item subjective self-report well-being Questionnaire (WQ) (Noon et al., 2015, Journal of Sports Sciences, 33, 2016-2115) countermovement jump (CMJ), resting heart rate $\left(\mathrm{HR}_{\text {rest }}\right)$, heart rate variability (HRV) were collected immediately prior to and $20 \mathrm{~h}$ following each trial. All participants and parents provided written informed consent following Institutional ethical approval. Paired t-tests were used to determine any differences between the low load and high load trials. Furthermore, confidence intervals and effect sizes using Cohen's $d$ were also calculated. To determine individual responses to the high load and low load trials, the likelihood of a change for each individual was assessed considering the typical error and smallest worthwhile change (Al Haddad, 2011, International Journal of Sports Medicine, 32, 598-605). Subjective self-report WQ items showed small to large deteriorations following the high load compared to low load $(d=$ $0.4-1.5, P=0.03-0.57)$. A very large increase in $\operatorname{HR}_{\text {rest }}(d=2.1, P$ $=0.02,95 \% \mathrm{Cl}: 1,10$ beats $\left.\cdot \mathrm{min}^{-1}\right)$, moderate decrease in HRV indices $(d=0.7, P=0.04,95 \% \mathrm{Cl}:-0.18,0.00 \mathrm{~ms}$ and $d=0.7, P=$ $0.01,95 \% \mathrm{Cl}:-0.21,-0.04 \mathrm{~ms}$ for In SDNN and rMSSD, respectively) and no change in countermovement jump $(d=0.0, P=$ $0.97,95 \% \mathrm{Cl}:-1.3,1.3 \mathrm{~cm}$ ) were evident when the high load was compared to low load. Individual WQ responses revealed $7 / 9$, $7 / 9,6 / 9,6 / 9,5 / 9,3 / 9$ and $1 / 9$ participants reported deteriorations in recovery, sleep quality, motivation, muscle soreness, fatigue, stress and appetite, respectively, following the high load compared to low load. Individual analysis indicated a negative response following the high load compared to low load in $\mathrm{HR}_{\text {rest, }}$ In SDNN and In rMSSD for 4/6, 2/6 and $1 / 6$ participants, respectively. Selected WQ items detected group and individual responses to high load and low load highlighting their potential utility. However, objective assessments lacked the sensitivity to detect small individual changes. 
D2.P54. The effect of ego depletion on challenge and threat states and performance under pressure

\section{JESSICA O'BRIEN ${ }^{1 *}$, LEE MOORE ${ }^{2}$, SIMON FRYER ${ }^{1}$ \& JOHN PARKER ${ }^{1}$}

\section{${ }^{1}$ University of Gloucestershire, ${ }^{2}$ University of Bath *Corresponding author: jmsobrien23@gmail.com}

A challenge state benefits pressurized sports performance more than a threat state (Moore et al., 2012, Psychophysiology, 49, 1417-1425). However, to date, little research has investigated the factors that influence these states. One such factor is ego depletion, or a reduction in self-control resources, which has been shown to have a detrimental effect on the performance of subsequent self-control tasks (Englert \& Bertrams, 2012, Journal of Sport and Exercise Psychology, 54, 580-599). Therefore, this study investigated the effects of ego depletion on challenge and threat states and performance during a pressurized muscular endurance task that required self-control. On approval of institutional ethics, 58 participants (mean age: 27.79 \pm 11.79 years) were randomly assigned to either an ego depletion or control group. Participants completed two trials of an isometric handgrip task (40\% of maximum voluntary contraction), separated by a transcription task. This task required selfcontrol for the ego depletion group (omit letters " $e$ " and " $n$ "), but not the control group (copy text in full). Before the second handgrip task, participants received instructions to elevate pressure, and then challenge and threat states were assessed via selfreport (demand resource evaluation score), and cardiovascular reactivity (challenge/threat index: as Moore et al., 2013, Journal of Sport and Exercise Psychology, 35, 551-562). The results revealed that the transcription task required more self-control for the ego depletion group than the control group, with the latter transcribing fewer words $(73.92 \pm 19.86$ vs $110.78 \pm 16.74$, $P<.001, d=-2.00)$, and making more errors ( $10.42 \pm 4.12$ vs. 4.13 $\pm 2.69, P<.001, d=1.80$ ). Furthermore, the ego depletion and control groups did not differ in terms of challenge and threat states, either in terms of demand resource evaluation score $(0.34$ \pm 0.93 vs. $0.00 \pm 1.03, P=.201, d=0.34)$, or challenge/threat index $(-0.04 \pm 0.99$ vs. $-0.38 \pm 0.96, P=.256, d=0.34)$. Moreover, the groups did not differ in terms of change in endurance performance from the first to second trial of the handgrip task ( $4.21 \pm 92.62$ s vs. $16.35 \pm 64.00$, seconds, $P=.571, d=-0.15)$. The findings suggest that ego depletion does not influence challenge and threat states or performance during a pressurised sporting task, adding to recent research questioning the existence of the ego depletion effect (Hagger et al., 2016, Perspective on Psychological Science, 11, 546-573).

D2.P55. Multiple single-subject approach: running pace and step characteristics among 400-m hurdlers

\section{MITSUO OTSUKA* \& TADAO ISAKA}

\section{Ritsumeikan University \\ *Corresponding author: otsuka-a@st.ritsumei.ac.jp}

The purpose in this study was to investigate the running pace and step characteristics among the world-class 400-m hurdlers so as to understand whether the elite hurdlers are individually more reliant on first-part pace or latter-part pace during the race and more reliant on step frequency (SF) or step length (SL). The total number of analysed races were 232 from publicly available television broadcasts, with twelve hurdlers performing in $17.8 \pm 4.3$ races. For each run of each hurdlers, finish time $(\mathrm{T})$, the rap time (RT) during the first and latter parts in 400-m hurdles races, the average SF and average SL were analysed with institutional ethics approval. The first and latter parts in races were divided at the instant of touchdown during the 5th hurdle clearance (almost $185 \mathrm{~m}$ from a start line). To determine any first-part or latter-part RT reliance and SF or SL reliance for an individual hurdler, the $90 \%$ confidence intervals $(\mathrm{Cl})$ for the difference between the first-part RT-T versus latter-part RT-T relationships and between the difference between the SF-RT versus SL-RT relationships was derived using a criterion nonparametric bootstrapping technique. Seven hurdlers performed these races with a latter-part RT reliance. The largest negative difference (latter-part RT reliance) was -0.50 ( $\mathrm{Cl}$ range: -0.93 to -0.20 ). In contrast, the highest positive difference (first-part RT reliance) was a 0.10 ( $\mathrm{Cl}$ range: -0.09 to 0.29$)$ and no hurdlers performed these races with a first-part RT reliance. Also, hurdlers performed these races with various combinations of SF and SL reliance during the first and latter parts. During the first part in the race, eight hurdlers performed these races with a SF reliance and the largest negative difference (latter-part reliance) was $-1.00(\mathrm{Cl}$ range: -1.00 to -1.00 ). During the latter part in the race, the number of hurdlers with a SF reliance decreased to two, most of hurdlers did not yield either reliance. These multiple single-subject approaches suggest that individual glycolytic and aerobic capacities contribute to high performance in long sprint event and this could be evaluated during the step frequency during the latter part in the race.

\section{D2.P56. Internal and external responses to linear versus multi-directional running}

\section{CHELSEA OXENDALE*, JAMIE HIGHTON \& CRAIG TWIST}

\section{University of Chester}

${ }^{*}$ Corresponding author: c.oxendale@chester.ac.uk @ChelseaOxendale

An individual's physiological response to running is dependent on the number of directional changes performed (Hader et al., 2014, BMC Sports Science, Medicine and Rehabilitation, 6. doi:10.1186/2052-1847-6-2). However, the utility of available measures of external and internal intensity during linear and multi-directional running remains unclear. Thus, the purpose of the study was to examine internal and external measures during linear and multi-directional running over the same distance. After institutional ethics approval, twelve team sport athletes (age: $20.8 \pm 2.7$ years; stature: $1.76 \pm 0.12 \mathrm{~m}$; mass: $73.3 \pm 12.8 \mathrm{~kg}$; $\mathrm{VO}_{2 \text { peak }}: 45.2 \pm$ $2.9 \mathrm{ml} . \mathrm{kg}^{-1} \cdot \mathrm{min}^{-1}$ ) completed a linear and multi-directional trial. Each trial consisted of $8 \times 175 \mathrm{~m}$ bouts of intermittent sprinting $(105 \mathrm{~m})$ and jogging $(70 \mathrm{~m})$ with $120 \mathrm{~s}$ of rest between bouts. Movement was quantified using a $10 \mathrm{~Hz}$ micro-technology device. Oxygen consumption $\left(\mathrm{VO}_{2}\right)$, blood lactate $(\mathrm{B}[\mathrm{La}])$, summated heart rate $(\mathrm{sHR})$ and rating of perceived exertion (RPE) were measured during each trial. Differences between trials (mean \pm SD) were assessed using 
magnitude based inferences. High speed distance (677.2 \pm 57.5 cf. $416.5 \pm 97.4$ m; ES; $\pm 90 \%$ Cl: $2.4 ; \pm 0.3$ ) was most likely higher during the linear trial, whereas time at high power $(118.4 \pm 11.7 \mathrm{cf} .111 .5 \pm 10.2 \mathrm{~s} ;-0.5 ; \pm 0.4)$ and the number of accelerations performed ( $48.3 \pm 21.5$ cf. $7.2 \pm 3.8$; $-1.7 ; \pm 0.4$ ) were likely to most likely higher during the multidirectional trial. $\mathrm{VO}_{2}\left(27.0 \pm 2.6 \mathrm{cf} .26 .0 \pm 1.9 \mathrm{ml.kg}^{-1} \mathrm{~min}^{-1}\right.$; $-0.4 ; \pm 0.5)$, sHR (78.1 \pm 10.5 cf. $69.1 \pm 10.1 ;-0.8 ; \pm 0.4), \mathrm{B}[\mathrm{La}]$ $\left(10.2 \pm 2.0\right.$ cf. $8.2 \pm 2.6$ mmol. $\left.\mathrm{L}^{-1} ;-0.9 ; 0.4\right)$ and RPE (15.7 \pm 1.1 cf. $14.9 \pm 1.1 ;-0.3 ; \pm 0.5$ ) were possibly to very likely higher during the multi-directional compared with the linear trial. The external to internal ratio using measures of high speed: sHR $(1.5 ; \pm 0.4)$, high speed: $\mathrm{VO}_{2}(1.7 ; \pm 0.6)$, high speed: $\mathrm{B}[\mathrm{La}]$ $(3.1 ; \pm 1.5)$ and high speed: RPE $(2.0 \pm 0.5)$ demonstrated very likely large differences between trials. Conversely, high power: summated $\mathrm{HR}(0.3 ; \pm 0.4)$, high power: $\mathrm{VO}_{2}(-0.16 ; \pm 0.6)$ and high power: RPE $(-0.05 ; \pm 0.5)$ demonstrated small to trivial differences between trials. More directional changes induce a greater physiological and perceptual demand, despite reducing high speed distance covered. Time at high power better reflects changes in internal intensity compared with time at high speed during running.

D2.P57. Within and between-level, cross-seasonal comparison of anthropometric, physical and psychological measures in 4 developmental levels of elite youth rugby union

\section{VICTORIA PENPRAZE ${ }^{1 *}$, JACK URQUHART ${ }^{1,2}$ \& DERRICK SPEIRS $^{2}$}

${ }^{1}$ University of Glasgow, ${ }^{2}$ Scottish Rugby Union

*Corresponding author:

Victoria.Penpraze@glasgow.ac.uk

@vikipen

Understanding the development of talented rugby union players in pathway and academy settings is important for continued preparation and support of players into the professional rugby environment (Kobal et al., 2016, Journal of Strength Conditioning Research, 30, 2433-2439). This study aimed to (i) compare anthropometric, physical and psychological capacities in Scottish Rugby Union (SRU) elite players between 4 developmental levels and (ii) compare within each level across the season. With institutional ethics approval, Under 16 (U16, $\mathrm{N}=18)$, Under 18 (U18, N $=16)$, academy stage $2(S t 2, N=9$ ) and academy stage 3 (St3, N = 12) players completed 3 testing blocks over 2016-2017 season (early, mid and late). Anthropometric (height, weight, percentage body fat, fat free mass (FFM), fat mass), speed (10 \& $30 \mathrm{~m}$ sprint, $10 \mathrm{~m}$ momentum), aerobic fitness (YoYo Intermittent Recovery Test-1), anaerobic endurance (Repeated Sprint Ability), power (counter movement jump (CMJ) and standing long jump (SLJ)), strength (3 repetition maximum (RM) squat, bench, chin), agility (505) and psychological (Sports Mental Toughness Questionnaire (SMTQ)) characteristics were completed. Early season, most statistically significant differences were between ST3 and U16/U18 in weight (95\% Confidence Intervals of difference $(2.1,29.5) \&(3.2,33.8))$ respectively, FFM $((1.4,23.0) \&(6.4,28.2)), \operatorname{CMJ}((8.0,18.6) \&(1.2,18.6))$, allometrically scaled $3 R M$ squats $((250.4,527.4) \&(175.6$,
483.8)), bench $((40.6,71.9) \&(28.4,61.8))$, chin $((84.5,297.9)$ $\&(73.1,296.4))$ and $10 \mathrm{~m}$ momentum $((14.1,169.4) \&(25.8$, 199.4)). St3 were faster over $30 \mathrm{~m}$ than U16 $(-0.53,0.04)$. Similar patterns were seen in mid and late season. Additionally in mid-season, St3 and St2 had significantly greater power than U16 (CMJ, $(4.3,19.3),(2.6,17.6))$ \& SLJ $(11.2,63.6),(6.6,65.6)$ respectively). St3 had greater MT than U16 $(0.1,6.0)$. Repeated measures ANOVA show no statistically significant changes for agility, speed, aerobic or anaerobic fitness across the season for any playing level although large effect sizes were seen. U16 significantly improved lower body power (SLJ, $(3.9,35.5)$ ) and 3RM bench $(2.0,16.7)$ across the season. MT improved for U18 $(0.1,6.0)$, St2 $(2.0,7.0)$ and St $3(0.1,16.0)$. As expected, St3 were stronger, had greater power and lean mass, however, without having greater agility, aerobic fitness or anaerobic endurance, than younger players. Where no differences were found between the academy and pathway levels, or no changes across season, even where differences would be expected (e.g. body fat), greater focus can be made on these aspects of development in training.

\section{D2.P58. The effect of 10 weeks of targeted physical training for High Speed Craft Users on physical and cognitive performance}

\section{DEBBIE RISIUS*, GABRIELLA MITCHELL, CLAIRE FORD, BETHANY ELLIS \& MIKE FETSCH}

\section{Defence Science and Technology Laboratory *Corresponding author: DJRisius@Dstl.Gov.UK}

Military injuries positively correlate with lost duty days, early attrition from service and diminished combat effectiveness. Exposure to Shock \& Vibration (S\&V), such as that experienced by personnel on high speed boats, increases injury risk by up to five times. The quality of physical training and musculoskeletal condition can influence injury risk, and is improved by implementing role-specific exercises. The Royal Navy developed a new targeted physical training programme for high speed boat users, aiming to reduce injury occurrence by specifically targeting common injury sites of $\mathrm{S} \& \mathrm{~V}$ exposure. The aim of this study was to investigate the effectiveness of the $S \& V$ training programme at improving physical and cognitive performance and reducing post-transit fatigue in Royal Marine coxswains over a 10 week period. With MoD ethics approval, a randomised controlled trial was conducted ( $\mathrm{n}$ = 20). Participants carried out 7 different physical and cognitive tests (Vertical jump, plank hold, grip strength, back strength, squat hold, N-back test, Stroop test) immediately before and after a standardised $3 \mathrm{~h}$ high speed boat transit. After 10 weeks the same procedures were followed to assess any changes in baseline or post-transit fatigue performance. Participants kept a training diary throughout the 10 week period and heart rate was monitored throughout the boat transits. The data show no difference between the control and intervention group as a result of the 10 week training period in either baseline physical or cognitive performance, heart rate during transit, or post-transit levels of fatigue $(P>.05)$. However, differences in post-transit fatigue were observed between 
the two test days for both groups in vertical jump height $(+7.5 \%)$, grip strength $(+12.0 \%)$, possibly due to transit differences. Qualitative results indicated that the will to follow such a training programme was limited in this population due to sporadic work schedules, effort to design the training plan, and high pre-existing fitness levels. Mobility and flexibility work were recommended by participants as a more appropriate injury prevention strategy for Royal Marine Coxswains. This study indicates that the new S\&V training programme did not improve the physical performance, cognitive performance, or fatigability of Royal Marine Coxswains. However, the S\&V training programme may be more effective amongst populations with a more routine work schedule and a lower overall fitness level.

\section{D2.P59. The effects of Internal versus external} attentional focus instructions on muscle activation and movement effectiveness during a barbell back squat

\section{MICHAEL STARKEY, MATTHEW SEDGWICK \& JON RADCLIFFE*}

\section{Leeds Trinity University \\ *Corresponding author: j.radcliffe@leedstrinity.ac.uk}

An external focus of attention (attention placed upon movement effects or the environment) compared to an internal focus of attention (attention placed upon own bodily movements) has been consistently associated with an enhanced performance through an increase in the effectiveness and efficiency of the movement in a range of sport and exercise related tasks (Wulf, 2013, International Review of Sport and Exercise Psychology, 6, 77-104). However, there is limited research examining the application of attentional focus on isotonic resistance exercise. Therefore, the aim of this study was to compare the acute effects of internal and external focus of attention on movement effectiveness (power) and efficiency (muscle activity) during the barbell back squat, a multi-joint resistance exercise. Following institutional ethical approval, eleven male undergraduate students with more than one year of resistance training experience (mean $\pm \mathrm{s}$, age: $21.5 \pm 1.3$ years; stature: $1.82 \pm 0.07 \mathrm{~m}$; body mass: 90.7 $\pm 16.3 \mathrm{~kg}$ ) performed six sets of four repetitions of a barbell back squat at $70 \%$ of their one-repetition maximum. The first three sets completed were with either an internal or external attentional focus and then the second three sets the opposite focus, decided through randomised ordering. Peak and average power during each repetition was determined using a linear position transducer and muscular activity, of the rectus femoris, vastus lateralis, vastus medialis and biceps femoris, was determined using surface-electromyography (EMG). The means across all repetitions in a condition was determined and a Paired T-test used to compare between conditions. Peak and average power was significantly greater in the external compared to internal focus condition by $25 \pm$ $20 \%$ (External: $1142 \pm 289 \mathrm{~W}$, Internal: $948 \pm 327 \mathrm{~W}, P<0.01$, $d=0.62$ ) and $10 \pm 6 \%$ (External: $573 \pm 137 \mathrm{~W}$, Internal: $522 \pm$ $137 \mathrm{~W}, P<0.01, d=0.37$ ) respectively. There were small but not significant decreases in peak EMG for the rectus femoris and vastus medialis and in integrated EMG for the rectus femoris, vastus medialis and biceps femoris during the lifts in the external compared to internal attentional focus condition ( $P>0.05, d=0.2-0.5$ ). The results suggest that an external focus of attention acutely improves movement effectiveness during a barbell back squat without any concomitant increase in muscle activation. Consequently, performance enhancement may come as a result of more coordinated motor unit recruitment patterns during an external focus.

\section{D2.P60. Reliability and validity of Garmin Vector power meter compared to the Cyclus 2 ergometer}

\section{BEN STEPHENSON*, MICHAEL HUTCHINSON, KEITH TOLFREY \& VICTORIA GOOSEY-TOLFREY}

\section{Loughborough University \\ *Corresponding author: B.T.Stephenson@lboro.ac.uk @BStephenson311}

Several portable pedal-based power meters, such as Garmin Vector (GV) and Look Keo, have recently been studied for their reliability and validity. However, none have been compared with the Cyclus 2 (C2), an electromagnetically braked ergometer commonly used for laboratory cycling tests. Thus, the aim of this study was to assess the reliability and validity of the Garmin Vector power meter in comparison to the C2 over a range of power outputs (PO). 14 male and 5 female recreationally active participants (age: $25 \pm 6$ years; body mass: $74.9 \pm 7.6 \mathrm{~kg}$; stature: $1.80 \pm 0.09 \mathrm{~m} ; \dot{V} \mathrm{O}_{2 \max } 50 \pm 9$ $\mathrm{ml} \cdot \mathrm{kg}^{-1} \cdot \mathrm{min}^{-1}$ ) (mean $\pm \mathrm{SD}$ ) undertook an incremental exercise test to exhaustion and three $10 \mathrm{sec}$ maximal sprints. The protocol was repeated two to seven days later to assess reliability. During the protocol, PO was recorded continuously via both devices to determine validity. Ethical approval was granted by the University's ethical committee. The mean coefficient of variation (CV) for GV PO was $2.1 \%$. There was a strong correlation between GV and C2 PO $(r=0.997, P<$ $0.001)$, however GV significantly underestimated $P O(z=$ $-12.655, P<0.001, E S=0.125)$, displaying a systematic error of $-8 \mathrm{~W}$ (95\% confidence interval $=-9$ to $-7 \mathrm{~W}$ ) and random error of $6 \mathrm{~W}$. The $95 \%$ limits of agreement were -19 to $3 \mathrm{~W}$. There was no heteroscedasticity or proportional bias in the PO offsets during the incremental test. During the sprints, there was a significant difference in $\mathrm{PO}(z=-8.394, P$ $<0.001$, ES $=0.720$ ) whilst offsets were heteroscedastic and bias was proportional. Despite a $-8 \mathrm{~W}$ offset between the two devices, it is possible to transform PO. Further, with the acceptable PO variation, the GV can be a useful tool to regulate and monitor cycling $P O$ to track changes in performance or quantify training load. Nonetheless, GV should not be used to monitor PO during maximal sprints due to evidence of proportional bias.

D2.P61. The order of the predictive runs significantly affects the parameters of the speedduration relationship

\section{CHRISTOPH TRISKA ${ }^{1 *}$, BERNHARD KOLLER ${ }^{1,2}$, HARALD $^{*}$ TSCHAN $^{1}$ \& BETTINA KARSTEN ${ }^{3,4}$}


${ }^{1}$ University of Vienna, ${ }^{2}$ Austrian Institute of Sports Medicine, ${ }^{3}$ LUNEX University, ${ }^{4}$ University of Greenwich ${ }^{*}$ Corresponding author: christoph.triska@univie.ac.at @christophtriska

To determine critical speed (CS) and the total distance covered above CS until task failure $\left(D^{\prime}\right)$, predictive trials are generally performed in the order from the longest to the shortest trial (Jenkins \& Quigley, 1992, Medicine and Science in Sports and Exercise, 24(11), 1283-1289). Using a single-visit approach, these predictive trials can be interspersed by 30 min passive rest to ensure a full reconstitution of $D^{\prime}$ (Galbraith et al., 2015, International Journal of Sports Physiology and Performance, 9(6), 931-935.). The aim of this study was to assess a potential effect of a different predictive trial order (i.e. from longest to shortest vs from shortest to longest) on $\mathrm{CS}$ and $D^{\prime}$. To determine $\mathrm{CS}_{1} / \mathrm{CS}_{2}$ and $D^{\prime}{ }_{1} / D^{\prime}{ }_{2}$ ten recreationally active participants performed two fieldtests. These field-tests comprised of three predictive timetrial runs which were between 2,5 , and $10 \mathrm{~min}$ in duration. One field-test was performed in a descending order (from shortest to longest; $C S_{1}$ and $D^{\prime}{ }_{1}$ ) and the second field-test was performed in an ascending order (from longest to shortest; $\mathrm{CS}_{2}$ and $D_{2}^{\prime}$ ). Field-tests were assigned in a random order and participants were asked to cover the greatest distance possible within the given times. A linear regression of speed vs inverse of time was used to resolve CS and $D^{\prime}$ (speed $=D^{\prime} \times 1 /$ time $\left.+\mathrm{CS}\right)$. All procedures were approved by the host institution's local ethics committee. $\mathrm{CS}_{1}$ and $\mathrm{CS}_{2}$ were $3.72 \pm 0.43 \mathrm{~m} \cdot \mathrm{s}^{-1}$ and $3.51 \pm 0.50 \mathrm{~m} \cdot \mathrm{s}^{-1}$, respectively, and $D^{\prime}{ }_{1}$ and $D^{\prime}{ }_{2}$ were $136.5 \pm 19.2 \mathrm{~m}$ and $190.5 \pm 20.1 \mathrm{~m}$, respectively. A paired-samples t-test revealed significant differences for CS $(P=0.001)$ and $D^{\prime}(P<0.001)$. Significant correlations between the two field-tests were found for CS ( $r$ $=0.963)$, but not for $D^{\prime}(r=-0.057)$. No significant differences in distance covered were found for the $5 \mathrm{~min}$ time-trial between the two field-tests $(P=0.823)$, but for the 10 and 2 min time-trial $(P=0.004$ and $P=0.006$ respectively). Present results suggest that the order of the predictive trials has a significant effect on CS and $D^{\prime}$. Starting with the longest predictive trial leads to a significant faster predicted $3000-\mathrm{m}$ time $(778 \pm 90 \mathrm{~s}$ vs $815 \pm 112 \mathrm{~s} ; P=0.010)$. While starting with the longer predictive trial consistently resulted in higher CS, such a trend was not evident for results of $D^{\prime}$, as $D^{\prime}$ showed no significant correlation. It is speculated that a 30-min passive rest might not be sufficient to fully recover from a preceding trial and subsequent trials are negatively affected by fatigue.

\section{D2.P62. Thinking aloud: an exploration of cognitions in professional snooker}

\section{JAMES WELSH*, STEPHEN DEWHURST \& JOHN PERRY}

\section{University of Hull \\ ${ }^{*}$ Corresponding author: j.c.welsh@hull.ac.uk @JayCWelsh}

Presently, there is a dearth of exploration into the cognitive processes of elite sports performers during real-time performance. Therefore, this study explored the cognitions of seven professional snooker players during real-time solo practice performance using a Think Aloud (TA) protocol. This involved players verbalizing their thoughts in their naturalistic practice environments. Player's verbalizations were recorded during each solo practice performance, transcribed verbatim, and analyzed via protocol analysis. Analyses revealed an array of stressors and coping strategies. Specifically, the results highlighted key stressor themes in relation to table conditions, distractions, and mistakes. While alternatively, shot preparation was essential to problemfocused coping, with rationalizing being integral to emotion-focused coping. Further, the results highlighted the visual-perceptual and cognitive expertise of players, with regards to identification of problem balls and cueball spatial awareness, insofar as unearthing the deliberate structure to practice routines. Future research should continue to dissect the sport- specific nuances that underpin real-time performance during practices, in order to further understand the dynamic interaction of cognition and performance. TA is an appropriate methodology to use in the domain-specific sport of snooker.

\section{D2.P63. Analysis of finalist swimmers in senior world championships with previous participation in junior category}

\section{INMACULADA YUSTRES AMORES*, DANIEL JUÁREZ, FERNANDO GONZÁLEZ-MOHINO \& JOSÉ MARÍA GONZÁLEZ RAVÉ}

\section{University of Castilla-La Mancha \\ *Corresponding author: inmaculada.yustres@uclm.es}

There are many factors affecting the development of talent in senior in swimming, being studied most of them for performance optimization. Focusing on the influence of performance in junior categories in obtaining results in senior swimming categories in World Championships, just one study has been founded (Yustres, Fernández, Martín, \& González-Ravé, 2017, Plos One. [publication ahead of print]. Having it as reference, characterization of these swimmers will be analyzed providing fully information for early specialization and talent detention. Therefore, the aim of this study was to analyze finalist swimmers from Senior World Championships with previous participation in Junior World Championships, adding the effect of different variables in the performance obtained. With institutional ethics approval, 719 finalist swimmers at the Senior World Championships 2007, 2009, 2011, 2013, 2015 comprised the database, of which just $17.1 \%$ participated previously in junior categories being our study sample. The study was based on the ordinal variable "position". An ordinal logistic regression model (OFR) proportional hazards was used for the evaluation of variable factors on since. Of the $17.1 \%$ data related to Junior swimmers, $44.7 \%$ were men; $62.6 \%$ remain one year of competition, being in the second year of maintenance just $28.5 \%$ and $8.1 \%-0.8 \%$ respectively in third and fourth year. Highlighting that $85.7 \%$ of men participated in 200 backstroke. Swimmers' percentage who reached top positions (1-3) turned out to be $32.5 \%$, however the last positions (6-8) would reach $47.9 \%$. Model determined that staying in the various championships was a significant factor $(P<0.05)$ in the achievements of getting 
better places. On the other hand, variables gender, event, style and country and theirs interactions were no significant $(P<0.05)$ with variable position as dependent. The analysis conclude that the number of swimmers who participate in junior categories prior senior in World Championships is quite sort. Having a high probability of repeating participation the second year but going it down in following years. Being the fact of participating in various championships a plus for achieving better positions. Besides, swimmers who participate in 200 backstroke in junior categories are more likely to repeat participation in senior categories. Therefore, participating in junior categories is recommended in order to remain no more than 3 years, being the positions obtained better each year. Regarding, 200 backstroke is the most recommended event to repeat participation in senior.

\section{D2.P64. The archetypes of masculinity and collective unconscious in elite English football academies}

\section{NICHOLAS ZUCH}

\section{University of Central Lancashire \\ *Corresponding author: nazuch@uclan.ac.uk @nickyzucho}

It is hypothesised that the human unconscious consists of two layers: the personal unconscious and collective unconscious (Jung, 1959, The archetypes and the collective unconscious. London: Routledge). Personal experience is theorised to define the former while inherent behavioural patterns, dubbed archetypes, form the latter, these being universal to all human minds. Previous studies reveal that individuals exhibiting mature archetypes, specifically the warrior archetype, can enhance development and performance in sport. (Moore \& Gillete, 1990, King, warrior, magician, lover: rediscovering the archetypes of the mature masculine. San Francisco: Harper Collins Publishers). The psychosocial journey from immature hero to mature warrior is conceptualised as a complex interweaving between an individual's collective unconscious, inherited self, and environment that is yet to be contextualised. Therefore, the aims of this study on talent development and performance in English football academies are to explore the implications of the immature and mature archetypes called the hero and the warrior, and their bipolar counterparts the bully and the coward, and the sadist and the masochist. This is with a view to exploring the impact of various talent development environments and coaching methods on the psychosocial development of football players. Following ethical approval, a two stage ethnographic study was conducted using an interpretist approach. Stage one was comprised of eight field observations (four per team) of an under nine and under ten team in a category three English football academy. In stage two semi-structured interviews with key academy staff members concerning their experiences with and perceptions of the participants and the club's talent pathway to establish major and minor themes. Results indicate that although they are considered psychosocially immature, participants were accessing the warrior/hero and their bipolar archetypes numerous times during one session/ game. Participants who displayed behaviours of the warrior and hero archetypes regularly were perceived by academy staff to be developing and performing more effectively in comparison to participants habitually accessing the bipolar archetypes. Results also show a potential link between the use of planned traumas in a situationalbased approach underpinned by a warrior/hero-based culture and effective development of these archetypes. Further research is required to fully comprehend the psychosocial maturation process from the immature to the mature warrior archetype and the mediators to psychosocial growth before stronger conclusions are made. Correspondingly, the researcher recommends that practitioners in English football academies identify the archetypes that an athlete is displaying and facilitate appropriate experiences to enhance development and performance. 


\section{Index}

\section{Key to abstract codes}

Unique codes. Each abstract has an unique code to assist you in identifying whether the abstract is a poster or free communication presentation and in which session it will be presented.

Page numbers. For each abstract a page number is provided, giving the location of where it is published in this booklet.

Poster presentations. There are two poster presentation sessions:

1. Day 1, 13:15-14:15

2. Day $2,12: 50-13: 45$.

Poster presentations have a code such as D2.P15 - Day 2, poster board number 15.

Free communication presentations. There are 10 free communication presentation sessions:

1. Day 1, 14:15-15:45 (5 parallel sessions)

2. Day 2, 11:05-12:20 (5 parallel sessions).

Free communication presentations have a code such as D2.S2.3(4) - Day 2, session 2.3, fourth presentation.

Abdullah, Nurul-Fadhilah, D1.P5, s21

Abt, Grant, D1.S3.5(1), s16

Adie, James W., D2.S1.4(5), s77

Akehurst, Sally, D1.P48, s42

Akhurst, Liam, D1.P10, s23

Akubat, Ibrahim, D1.S3.5(1), D2.S4.5(4), D2.S1.5(6), s16, s61, s81

Al Shehhi, Mouza, D1.P12, s24

Alamdari, Nima, D2.S1.1(4), s64

Alexandrova, Albena, D1.P20, s28

Allen, Answorth A., D1.P58, D2.S1.1(2), s46, s63

Allen, Danyelle, D1.P22, s30

Allen, Justine, D1.P32, s34

Aning, Jonathan, D1.P6, s21

Archer, David, D2.S4.5(1), s60

Arnold, Rachel, D1.S3.5(4), D2.P28, s17, s97

Ashford, Kelly, D2.S4.1(1), D2.S4.2(4), s48, s52

Ashton, Ruth, D1.P6, s21

Atkins, Steve, D1.P54, s44

Atkinson, Greg, D2.S4.2(5), s52

Avans, Diana, D1.P22, D2.P1, s30, s83

Backhouse, Susan H., D2.S3.2, D2.P31, s1, s98

Bailey, Daniel, D1.S3.1(1), s3

Baines, Josef D., D2.P32, s98

Baker, Julien S., D1.P21, s29

Balent, Boris, D1.P23, s30

Balk, Yannick, D2.S1.3(1), s71

Ballister, Jessica, D1.S3.4(4), s14

Balshaw, Thomas G., D1.S3.2(1), D2.S4.1(3), s7, s49

Barakat, Ruben, D2.P13, s88

Barnes, Andrew, D1.P1, s19
Barrett, Steve, D1.S3.5(1), s16

Basevitch, Itay, D1.P25, D1.P28, D1.P37, s31, s32, s36

Batterham, Rachel, D1.P18, s27

Bedford, Kieran, D1.P3, s20

Bigliassi, Marcelo, D1.S3.4(5), s14

Bishop, Nicolette, D1.S3.2(4), s8

Bitchell, Charlotte, D2.S4.2(4), s52

Björklund, Glenn, D1.P49, s42

Black, Matthew I., D2.P50, s107

Blackney, Phoebe, D2.P6, s85

Blaser, Marc, D2.S1.5(1), s79

Blijlevens, Suzan J.E, D1.P24, s31

Blundell, John, D2.P10, 587

Boal, Mariette, D1.P25, s31

Board, Elisabeth M., D1.S3.2(5), D1.P15, s9, s26

Boardley, Ian, D1.P40, D2.S3.2, s1, s38

Boat, Ruth, D1.P4, D1.P26, D2.P5, s20, s31, s85

Bolmont, Benoit, D2.P40, s102

Bonfanti, Noelia, D1.P16, s26

Bonner, Stuart, D1.P1, s19

Bool, Kayan, D1.P24, s31

Borkowski, Lech, D2.S1.5(4), s80

Born, Dennis, D1.P49, s42

Bortolini, Miguel Junior Sordi, D1.P36, s36

Bosnar, Ksenija, D1.P23, s30

Bott, Elly, D2.S4.2(1), s51

Bradley, Chris, D1.P27, s32

Bradley, Claire, D1.P13, s25

Bradley, Eddie, D2.S4.5(1), s60

Bradley, Helen E., D1.P5, s21

Bray, James, D1.S3.5(1), s16 
Breaden Madden, Gerard, D2.S1.3(5), s72

Bressan, Liz, D2.S4.3(1), D2.P52, s54, s108

Brewer, Deborah, D2.S4.4(1), s57

Brick, Noel, D1.S3.4(1), s13

Brimmell, Jack, D1.P50, s42

Broom, David, D2.S4.2(1), s51

Brown, Lorraine, D2.S4.4(4), s58

Broyles, Stephanie T., D1.S3.1(6), s5

Bruce, Andrew C., D1.S3.4(5), s14

Bruno, Pedro, D1.P28, s32

Burbage, Jenny, D2.S1.5(2), s79

Burleigh, Mia, D1.P21, s29

Burnet, Scott, D1.P51, s43

Burns, Stephen F., D1.S3.1(4), s4

Camiré, Martin, D2.P34, s99

Campbell, Mark J., D1.S3.3(1), D1.S3.4(1), D2.S4.3(1), D2.P52, s10, s13, s54, s108

Cantu, Robert C., D1.P58, D2.S1.1(2), s46, s63

Carlin, Liz, D1.P29, s33

Carnegie, Evelyn, D2.P38, s101

Catlow, Sarah, D1.P52, s43

Cavallerio, Francesca, D2.S4.4(2), s57

Celis-Morales, Carlos, D1.P14, D2.S1.2(7), D2.P14, s25, s69, s89

Chamorro, Jose L., D2.P49, s106

Champagne, Catherine M., D1.S3.1(6), s5

Chaput, Jean-Philippe, D1.S3.1(6), s5

Charnock, Lewis, D1.P30, s33

Chester, Neil, D2.S3.2, s1

Chua, Hong En Joshua, D2.P23, s93

Clarke, Neil D., D1.P19, D1.P53, D2.S4.1(2), D2.P11, D2.P53, s28, s44, s48, s87, s108

Clarke, Philip, D1.P48, s42

Cockburn, Emma, D1.P17, D2.S1.1(7), D2.P20, s27, s66, s92

Collazos, Carlos A., D1.S3.1(5), D2.P12, s5, s88

Collins, Helen, D1.P9, s23

Collins, Richard, D2.P30, s97

Comfort, Paul, D2.S4.5(3), s61

Comoutos, Nikos, D1.P44, s39

Comyns, Thomas, D2.S1.1(6), D2.S1.5(3), s65, s80

Condon, Tara, D1.P58, s46

Connaughton, Alison, D1.P7, s22

Conway, Becky, D1.P2, s19

Cook, Christian, D2.P19, s92

Cooke, Andrew, D1.S3.3(6), s12

Cooke, Carlton, D2.S1.1(1), s63

Cooley, Sam, D2.S1.2(5), s68

Cooper, Christopher, D1.P10, s23

Corbett, Jo, D1.S2.2, s1

Coussens, Adam, D1.P31, s34

Coyle, Melissa, D1.S3.3(2), s10

Crewther, Blair, D2.S1.5(4), D2.P19, s80, s92

Cromwell, Jim A., D2.P32, s98

Cronin, Lorcan, D1.P32, D2.P38, s34, s101

Cullen, Matthew, D1.S3.4(2), s13

Cumming, Jennifer, D2.S1.2(5), s68

Cumming, Sean, D1.S3.1(6), s5

Currell, Kevin, D2.S3.2, s1

Cury, François, D2.P8, s86

Cvijanovic, Tamara, D1.P23, s30

Dallaway, Neil, D1.S3.5(2), s16

Dalton, Michelle, D2.P10, s87
Danish, Steve, D2.S4.4(5), s58

Davenport, Andrew D., D2.S1.1(4), s64

Davies, Emma, D2.P29, D2.P30, s97

Davies, Simeon, D2.S1.1(1), s63

De Brandt, Koen, D1.P33, D1.P47, s35, s41

De Jonge, Jan, D2.S1.3(1), s71

De Muynck, Gert-Jan, D1.S3.4(3), s13

De Vivo, Marlize, D1.P8, s22

Deane, Frank, D1.S3.3(4), s11

Defruyt, Simon, D1.P34, D1.P47, s35, s41

Degraeuwe, Lotte, D1.S3.4(3), s13

Dekerle, Jeanne, D2.P22, s93

Delallo, Jessica F., D2.S1.1(2), s63

Denstel, Kara D., D1.S3.1(6), s5

Dewhurst, Stephen, D2.P62, s112

Diaz, Jaime, D1.S3.4(2), s13

Dimakopoulou, Eleni, D2.S1.1(3), s64

Dimitriou, Lygeri, D1.P17, D2.S1.1(7), D2.P20, s27, s66, s92

Dobbin, Nick, D2.S4.5(2), s60

Doggart, Lance, D1.P52, s43

Dorling, James, D1.P18, D2.S4.2(5), s27, s52

Duncan, Audrey, D1.P9, s23

Duncan, Michael J., D1.S3.1(2), D1.P4, D1.P10, D1.P26, D1.P57, D2.S1.2(4), D2.S1.2(6), D2.P5, D2.P9, D2.P11, D2.P15, D2.P25, s3, s20, s23, s31, s46, s68, s69, s85, s86, s87, s89, s94

Dutton, Paul, D1.P46, s40

Easton, Chris, D1.P21, s29

Eccles, David, D2.S4.4(3), s58

Eccles, Nicola, D2.S1.2(3), s68

Economou, Marina, D1.P44, s39

Edwards, Laura, D2.P2, s83

Edwards, Thomas, D2.P28, s97

Egginton, Stuart, D2.P6, s85

Elbe, Anne-Marie, D2.S1.3(3), s72

Elferink-Gemser, Marije T., D1.P24, s31

Ellis, Bethany, D2.P58, s110

Ellis, Matthew, D1.P19, D1.P53, s28, s44

Ellison, Paul, D1.P32, D2.P38, s34, s101

Engels, Eliane Stephanie, D2.S1.4(1), s75

Ennis, Julia, D2.P30, s97

Esen, Ozcan, D2.S1.1(5), s65

Esteban Montes, Jeisson, D2.P12, s88

Eyre, Emma, D1.S3.1(2), D1.P10, D1.P26, D2.P5, D2.P9, D2.P15, s3, s23, s31, s85, s86, s89

Ferguson, Helen, D1.S3.3(5), D2.S1.4(2), s12, s75

Fernandes, John F. T., D1.S3.2(2), s7

Fetsch, Mike, D2.P58, s110

Filho, Edson, D2.S4.3(5), s55

Finlayson, Graham, D2.P10, s87

Finn, Charlie, D2.P19, s92

Fitzgerald, Evan, D2.S1.5(3), s80

Flanagan, Eamonn, D2.S1.5(3), s80

Fleming, Sean, D2.S1.5(3), s80

Flood, Danielle, D1.P54, s44

Fogarty, Andrea, D1.S3.3(5), s12

Fogelholm, Mikael, D1.S3.1(6), s5

Folland, Jonathan P., D1.S3.2(1), D2.S4.1(3), s7, s49

Ford, Claire, D2.P58, s110

Forsdyke, Dale, D2.S1.1(6), s65

Fountoulakis, Charalampos, D1.S3.4(2), s13 
Fraser, Jonathan, D1.P1, s19

Fraser, Kotryna, D1.S3.3(3), D1.P35, s11, s36

Freeman, Jasmine, D1.P22, s30

Freeman, Paul, D2.S4.3(2), D2.P50, s54, s107

Freitas Silva, Carolina, D1.P36, s36

French, Christa, D2.P1, s83

Freund, Philipp Alexander, D2.S1.4(1), s75

Fritsch, Julian, D2.S1.3(3), s72

Fryer, Nina, D1.P46, s40

Fryer, Simon, D2.P54, s109

Fu, Di, D2.S4.3(2), s54

Fujita, Satoshi, D2.P27, s95

Furusa, Maita, D1.P37, s36

Gallacher, Michael, D1.P9, s23

Gamieldien, Raeeq, D2.S1.1(1), s63

Garcia, Jose L., D1.S3.1(5), D2.P12, s5, s88

Garrote Filho, Mario Da Silva, D1.P36, s36

Gennuso, Gabriel, D1.S3.2(6), s9

Geurts, Sabine, D2.S1.3(1), s71

Gibbons, Catherine, D2.P10, s87

Gibson, Kass, D1.S3.3(2), s10

Gibson, Oliver, D1.S2.2, s1

Gilbert, Stephen, D1.P6, s21

Gill, Jason, D1.P14, D2.S1.2(7), D2.P14, s25, s69, s89

Gillison, Fiona B, D1.S3.1(6), s5

Gittoes, Marianne, D2.S4.1(1), D2.S4.2(4), s48, s52

Glynn, Jonathan, D1.S3.4(2), s13

Godin, Philippe, D2.S1.4(7), s78

Golding, Danny, D1.P38, D2.S1.4(3), s37, s75

Goltsi, Vicky, D1.P39, s37

Gondek, Marcella, D2.S1.1(7), D2.P20, s66, s92

González Ravé, José María, D1.P55, D2.P63, s45, s112

González-Mohíno, Fernando, D1.P55, D2.P63, s45, s112

Goosey-Tolfrey, Victoria, D2.P60, s111

Gorczynski, Paul, D1.S3.3(2), s10

Goulding, Richie, D1.S3.2(3), s8

Graham, Catherine, D2.P6, s85

Grainger, Adam, D2.S4.5(3), s61

Grant, Kirsten, D1.S3.5(6), s18

Gray, Stuart, D1.P14, D2.S1.2(7), D2.P14, s25, s69, s89

Groves, Scott, D2.P48, s106

Gutierez, Adriana R., D1.S3.1(5), D2.P12, s5, s88

Hagger, Martin S., D2.S1.3(2), s71

Hainaut, Jean-Philippe, D2.P40, s102

Haines, Matthew, D1.S3.1(3), s4

Haleem, Samuel Y., D2.S1.1(2), s63

Hamer, Mark, D1.S3.4(5), s14

Harper, Damian, D2.S1.1(6), D2.S1.5(3), s65, s80

Harwood, Chris, D1.P32, s34

Hassan, David, D1.P29, s33

Hatfield, Johannes L., D2.P35, s100

Hatzigeorgiadis, Antonis, D1.P44, D2.S1.3(3), s39, s72

Havenhand, Louis, D2.S4.2(1), s51

Hawkey, Adam, D1.P2, D1.P3, D2.P2, D2.P4, s19, s20, s83, s84

Hayes, Mark, D2.P22, s93

Hazelwood, James, D2.S1.2(1), s67

Heijboer, Mathieu, D1.S3.5(5), s18

Henderson, Ginny, D1.P9, s23

Henderson, Jennifer, D2.S4.3(3), s54

Hendricks, Sharief, D1.S3.4(6), s15
Henselmans, Menno, D2.P21, s93

Herring, Matthew P., D1.S3.3(1), s10

Hettinga, Florentina, D1.S3.5(3), D1.P59, s17, s47

Heyes, Andrew, D1.P40, s38

Highton, Jamie, D2.S4.2(2), D2.S4.5(2), D2.S4.5(5), D2.S1.5(5),

D2.P56, s51, s60, s61, s80, s109

Hill, Mathew, D1.P4, D2.S4.2(3), s20, s52

Hill, Timothy, D1.P15, s26

Hills, Frank, D1.P17, D2.S1.1(7), D2.P20, s27, s66, s92

Hinchliffe, Charlotte, D2.P31, s98

Hobbs, Sarah, D1.P54, s44

Hockey, Pierre, D1.P3, s20

Hodge, Alex, D2.S4.4(3), s58

Hodge, Ken, D2.S4.4(5), s58

Hoekstra, Sven, D1.S3.2(4), s8

Hogg, Bob, D2.S4.5(1), s60

Holder, Tim, D2.P39, s102

Holland, Mark, D2.S1.2(5), s68

Holloway, Kathryn, D1.P11, s24

Hori, Miyuki, D2.P27, s95

Horne, Mark, D1.P56, s45

Howat, Douglas, D2.S1.4(5), s77

$\mathrm{Hu}$, Gang, D1.S3.1(6), s5

Hudson, Amanda, D2.S3.2, s1

Hudson, Sean, D2.S1.1(1), s63

Hughes, David, D1.P21, s29

Hume, Patria A., D1.P58, D2.S1.1(2), s46, s63

Huntley, Emma, D2.P38, s101

Hurst, Josh, D1.P4, D1.P26, s20, s31

Hutchinson, Jasmin, D1.S3.4(4), s14

Hutchinson, Michael, D2.P60, s111

Isaka, Tadao, D2.P27, D2.P55, s95, s109

Ishihara, Toru, D2.P51, s107

James, Lewis, D2.S4.2(1), s51

James, Rob S., D2.S1.2(6), D2.P53, s69, s108

Jei Ni Sim, D2.P23, s93

Jimenez, Alfonso, D2.P11, s87

Johnston, Richard, D1.P12, s24

Johnstone, James, D2.P21, s93

Jones, Ben, D2.P31, s98

Jones, Ian, D2.S4.4(4), s58

Jones, Jenny, D1.P18, s27

Jones, Martin I., D2.P42, s103

Jones, Martyn, D1.P9, s23

Jones, Paul, D2.S4.5(3), s61

Jones, Victoria, D1.P26, D2.P5, D2.S4.1(2), D2.P11, s31, s85, s48, s87

Jordana, Anna, D2.P49, s106

Juárez, Daniel, D1.P55, D2.P63, s45, s112

Juris, Paul, D2.P11, s87

Kajtna, Tanja, D1.P41, s38

Kaloupsis, Sokratis, D2.S1.1(3), s64

Kamijo, Yoshi-Ichiro, D1.S3.2(4), s8

Kanehisa, Hiroaki, D2.S4.1(4), s49

Karageorghis, Costas I., D1.S3.4(5), D2.P32, s14, s98

Karsten, Bettina, D2.P61, s111

Kashiwabara, Kyoko, D1.S3.1(4), s4

Katzmarzyk, Peter T., D1.S3.1(6), s5

Kavanagh, Emma, D2.S4.4(4), D2.P41, s58, s103 
Kawakami, Yasuo, D2.S4.1(4), s49

Keegan, Richard, D1.S3.3(5), s12

Kegelaers, Jolan, D1.P42, s39

Kelly, Adam, D2.P33, s99

Kelly, Seamus, D1.S3.5(4), s17

Kendellen, Kelsey, D2.P34, s99

Kenny, Ian, D2.S4.3(1), D2.P52, s54, s108

Kerrigan, Alex, D2.S4.2(1), s51

Kidokoro, Tetsuhiro, D1.S3.1(4), s4

Kilduff, Liam, D2.P19, s92

King Chung Chan, Derwin, D1.P43, D2.S1.3(2), s39, s71

Kilgallen, Conor, D1.S3.4(2), s13

King, Anne, D2.P6, s85

King, James, D1.P18, D2.S4.2(5), s27, s52

King, Lewis, D1.P31, s34

Kinman, Gail, D1.P38, D2.S1.4(3), s37, s76

Kinoshita, Tokio, D1.S3.2(4), s8

Knight, Camilla, D2.S1.4(6), s77

Kolimechkov, Stefan, D1.P20, s28

Koller, Bernhard, D2.P61, s111

Kollias, Iraklis A., D2.S1.1(3), s64

Konings, Marco, D1.S3.5(3), s17

Kourbetis, Dimitrios, D2.S1.1(3), s64

Kozub, Steve, D1.P38, D2.S1.4(3), s37, s76

Kristjansdottir, Hafrun, D2.S1.3(7), s73

Kuriyan, Rebecca, D1.S3.1(6), s5

Kurpad, Anura V., D1.S3.1(6), s5

Kwan Xue Bin, Kedric, D2.P20, s92

Laborde, Sylvain, D2.P41, s103

Lacouchie, Héloïse, D2.S1.3(4), s72

Lafferty, Moira E., D2.P36, s100

Lahart, Ian, D2.S1.2(2), s67

Lamb, Kevin L., D1.S3.2(2), s7

Lambert, Estelle V., D1.S3.1(6), s5

Lanza, Marcel B., D1.S3.2(1), D2.S4.1(3), s7, s49

Larmour, Rae, D2.P6, s85

Laroche, Manon, D2.P8, s86

Latinjak, Alexander T., D2.S4.3(4), D2.P44, D2.P49, s55, s104, s106

Lawson, Chelsey, D1.S3.1(2), D1.P26, D1.P57, s3, s26, s46, D2. P5, s85

Lea, John W. D., D2.P7, s86

Leckey, Stephen, D2.P37, s101

Lee, Joshua, D2.S4.5(5), s61

Leicht, Christof, D1.S3.2(4), s8

Leurquin, Anne-Sophie, D2.S1.4(7), s78

Levy, Andrew, D1.P32, s34

Lewis, Kiara, D2.S1.2(3), s68

Lewis, Liane, D1.P6, s21

Li, Hui, D1.P14, D2.S1.2(7), D2.P14, s25, s69, s89

Liddle, Sarah, D1.S3.3(4), s11

Littlewood, Martin, D1.P30, s33

Lloyd, Ray, D2.S1.1(1), s63

Lomax, Mitch, D2.S1.5(2), s79

Lonsdale, Chris, D2.S1.4(2), s75

Lopez Jr, Victor, D1.P58, D2.S1.1(2), s46, s63

Lories, Guy, D2.S1.4(7), s78

Lorimer, Ross, D2.P46, s105

Loukadakis, Manolis, D1.P44, s39

Lovell, Geoff P., D2.P42, s103

Low, Chris, D2.S1.1(1), s63

Lucas, Sam, D1.S3.5(2), s16

Lyons, Mark, D1.P57, s46
Ma, Richard, D1.P58, D2.S1.1(2), s46, s63

Macfarlane, Niall, D2.P37, s101

Macintyre, Tadhg, D1.S3.4(1), s13

Macquet, Anne-Claire, D2.S1.3(4), s72

Macutkiewicz, David, D2.P24, s94

Maden-Wilkinson, Thomas M., D1.S3.2(1), s7

Madigan, Daniel, D2.S4.3(2), s54

Maeo, Sumiaki, D2.S4.1(4), s49

Maher, Carol, D1.S3.1(6), s5

Maia, José, D1.S3.1(6), s5

Makramalla, Kerollos, D2.P1, s83

Malkin, Valerii, D2.P45, s104

Manley, Andrew, D2.P31, s98

Manolopoulos, Konstantinos N., D1.P5, s21

Marchant, David, D1.P32, D2.P38, s34, s101

Maridakis, Alexandros, D1.P44, s39

Marsden, Bradley, D2.P3, s84

Marshall, Ryan, D1.S3.2(5), s9

Martin, Emily, D2.P39, s102

Martins Silva, Romeu Paulo, D1.P36, s36

Marwood, Simon, D1.S3.2(3), s8

Maskus, Christin, D2.P26, s95

Massey, Garry J., D1.S3.2(1), s7

Mastagli, Maxime, D2.P40, s102

Masters, Rich, D2.P47, s105

Mathers, John, D2.S1.4(4), s76

Matsudo, Victor, D1.S3.1(6), s5

Matthews, James, D1.S3.5(4), s17

Maxwell, Neil, D1.S2.2, s1

May, Linda, D2.P13, s88

Maylor, Benjamin, D1.S3.1(1), s3

McConnachie, Thomas, D1.P9, s23

McKernie, Jed, D1.S3.5(6), s18

McMahon, John, D2.S4.5(3), s61

McSwiggan, Linda, D1.P9, s23

Megagiannis, Konstantinos, D1.P39, s37

Mel, Carlos E., D2.P12, s88

Mellalieu, Stephen, D1.S3.4(6), D1.P7, s15, s22

Metsios, George, D2.S1.2(2), s67

Michelmore, Charlotte, D2.S4.1(1), s48

Mikus, Catherine R., D2.S1.1(4), s64

Mills, Chris, D2.S1.5(2), s79

Mills, Hayley, D1.P8, s22

Mills, Kate, D2.S3.2, s1

Mire, Emily F., D1.S3.1(6), s5

Mitchell, Gabriella, D2.P58, s110

Miyashita, Masashi, D1.S3.1(4), s4

Molan, Conor, D1.S3.5(4), s17

Moore, Isabel, D2.S4.1(1), D2.S4.2(4), s48, s52

Moore, Lee J., D1.P50, D2.S1.3(6), D2.P50, D2.P54, s42, s73, s107, s109

Moran, Aidan, D2.S4.3(1), s54

Morita, Noriteru, D2.P51, s107

Morres, loannis, D1.P44, s39

Morris, Martyn, D1.P26, s31

Morris, Rob, D1.P30, s33

Mosley, Emma, D2.P41, s103

Moss, Samantha, D2.S4.5(2), s60

Muggeridge, David J., D1.P21, s29

Mulcahy, Amber, D2.P18, s90

Mullen, Richard., D2.P32, s98

Mullen, Thomas, D2.S1.5(5), s80 
Mulvenna, Mairi, D2.S1.4(5), s77

Mundy, Peter, D1.P53, D2.S4.1(2), D2.P9, s44, s48, s86

Murray, Kelly, D1.P59, s47

Murray, Mark, D1.S3.5(6), s18

Musatafa, Syakirah B., D2.P23, s93

Myers, Anna, D2.P10, s87

Myers, Tony, D1.S3.5(1), D2.S1.5(6), s16, s81

Nakajima, Toshihiro, D2.P5, s107

Nesti, Mark, D1.P30, s33

Newhouse, Safron, D1.P11, s24

Newport, Rachael, D2.S1.4(6), s77

Nicholas, Ceri, D2.S1.1(5), s65

Nicholson, Graham, D1.P12, s24

Ni Sim, Jei, D2.P23, s93

Niven, Ailsa, D1.P13, s25

Noetel, Michael, D1.S3.3(5), D2.S1.4(2), s12, s75

Noon, Mark R., D1.P19, D1.P53, D2.P53, s28, s44, s108

Nowlan, Gerard, D2.S4.2(2), s51

Ntallas, Georgios, D2.S4.1(5), s49

Nunns, Eleanor, D2.P6, s85

O'Brien, Jessica, D2.P54, s109

O'Driscoll, Jamie M., D2.P7, s86

Obmiński, Zbigniew, D2.S1.5(4), s80

Oerlemans, Wido, D2.S1.3(1), s71

Olds, Tim, D1.S3.1(6), s5

Oliver, Emily, D2.S4.4(3), s58

Ong, Chong Hao Nathanael, D2.P23, s93

Onywera, Vincent, D1.S3.1(6), s5

Orton, Charlie, D1.S3.1(1), s3

Osler, Callum, D2.P17, s90

Otsuka, Mitsuo, D2.P55, s109

Otsuka, Shun, D2.S4.1(4), s49

Outram, Tom, D2.P17, s90

Oxendale, Chelsea, D2.P56, s109

Oxford, Samuel, D2.S4.1(2), D2.S1.2(4), D2.P9, s48, s68, s86

Pabbathi, Vijay, D2.P26, s95

Pack, Stephen, D2.P46, s105

Page, Abigail, D2.P17, s90

Paice, Katherine, D2.P20, s92

Paice, Kathryn, D1.P17, s27

Pallares, Susana, D2.P49, s106

Panoutsakopoulos, Vassilios, D2.S1.1(3), s64

Parker, John K., D1.P50, D2.P42, D2.P50, D2.P55, s42, s103, s107, s109

Parry, Benjamin, D2.S1.2(5), s68

Peart, Daniel, D1.S3.5(1), s16

Pelaez, Mireia, D2.P13, s88

Pell, Jill, D1.P14, D2.S1.2(7), D2.P14, s25, s69, s89

Penha Silva, Nilson, D1.P36, s36

Penpraze, Victoria, D2.P37, D2.P57, s101, s110

Perlo, Merlin, D2.P29, s97

Perry, John, D2.P62, s112

Petermann, Fanny, D1.P14, D2.S1.2(7), D2.P14, s25, s69, s89

Petrov, Lubomir, D1.P20, s28

Phillips, Shaun, D1.P13, s25

Picknell, Gareth, D1.P12, s24

Pikouli, Katerina, D1.P44, s39

Plath, Christina, D1.P45, D2.P43, s40, s103

Ploumpidis, Dimitrios, D1.P44, s39

Pollitt, Lysander, D2.P3, s84
Pons, Joan, D2.P44, s104

Pop, Serban, D2.S4.2(2), s51

Pottratz, Suzanne T., D1.S3.4(5), s14

Price, Mike, D1.S3.2(6), D2.S4.2(3), s9, s52

Prot, Sara, D1.P23, s30

Pucci, Andrea, D1.P18, s27

Puddiford, Michael, D2.S4.2(3), s52

Quinton, Mary, D2.S1.2(5), s68

Radcliffe, Jon, D1.P46, D2.P59, s40, s111

Radesca, Krizia, D1.P16, s26

Ramis, Yago, D2.S4.3(4), D2.P44, D2.P49, s55, s104, s106

Reistenbach Goltz, Fernanda, D2.S4.2(5), s52

Ramos De Arvelos, Ramos, D1.P36, s36

Raymond, Leanne, D1.P4, D1.P26, D2.P5, s20, s31, s85

Rees, Tim, D2.S4.3(2), D2.P28, s54, s97

Rhoden, Clare, D2.S4.4(1), s57

Rice, Joshua, D1.P60, s47

Richardson, Darren, D2.P11, s87

Ring, Christopher, D1.S3.5(2), D2.S4.3(3), s16, s54

Risius, Debbie, D2.P58, s110

Riveros, Manuel A., D1.S3.1(5), D2.P12, s5, s88

Robbins, Daniel, D1.P3, s20

Roberts, Justin, D2.P21, s93

Roberts, Ross, D1.S3.3(6), s12

Robinson, Scott R., D1.P5, s21

Roche, Denise, D1.S3.2(3), s8

Rodriguez, Angela Maria, D2.P12, s88

Rogaleva, Liudmila, D2.P45, s104

Roldan-Reoyo, Olga, D2.P13, s88

Roohaizad, Nur Shakirah B., D2.P23, s93

Rooney, Kevin D., D1.P21, s29

Roscoe, Clare, D2.S1.2(6), s69

Ross, Christopher, D2.S1.2(4), s68

Rouquette, Olivier, D2.S1.4(7), s78

Roussel, Peggy, D2.P8, s86

Rubiano, Oscar F., D1.S3.1(5), D2.P12, s5, s88

Sagarra, Manuel Lapuente, D1.S3.5(1), s16

Sagawa, Masato, D2.P51, s107

Sage, Luke, D2.S1.4(5), s77

Sanders, Dajo, D1.S3.5(5), D2.S4.5(4), D2.S1.5(6), s18, s61, s81

Sarkar, Mustafa, D1.S3.3(5), D2.S1.3(6), s12, s73

Sarmiento, Olga L., D1.S3.1(6), s5

Sattar, Naveed, D1.P14, D2.S1.2(7), D2.P14, s25, s69, s89

Saxton, John, D1.P6, s21

Schäfer, Lisa, D2.P22, s93

Schipper-Van Veldhoven, Nicolette, D1.P34, s35

Scholefield, Elizabeth, D2.P46, s105

Schweickle, Matthew, D1.S3.3(5), D2.P48, s12, s106

Scott, Phil, D2.P19, s92

Sedgwick, Matthew, D2.P59, s111

Seiler, Roland, D2.S1.5(1), s79

Sellars, Paul, D1.S3.4(6), s15

Selwanes, Veronica, D2.P1, s83

Shafizadeh, Mohsen, D1.P1, s19

Shahvail, Moji, D1.S3.3(3), s11

Shan, Xiyao, D2.S4.1(4), s49

Sharp, Lee-Ann, D2.S4.4(5), s58

Sharpe, Phil, D2.S4.5(1), s60

Shearer, David, D2.S1.4(6), s77 
Sheehan, Rachel B., D1.S3.3(1), D1.S3.4(1), s10, s13

Sheffield, David, D1.P48, s42

Sheldon, William D., D1.S3.2(5), s9

Sherwood, Sebastian, D2.P47, s105

Sideri, Eirini, D1.P44, s39

Sillars, Anne, D1.P14, D2.S1.2(7), D2.P14, s25, s69, s89

Sinclair, Scott, D2.P20, s92

Sing Yeung Lee, Alfred, D1.P43, s39

Sivaperuman, Parimala, D2.P23, s93

Smith, Lee, D2.P21, s93

Smith, Paul, D1.S3.2(6), s9

Smith, Tiaki, D2.P47, s105

Soenens, Bart, D1.S3.4(3), s13

Sombra Silva, Igor, D1.P36, s36

Speirs, Derrick, D2.P57, s110

Stambulova, Natalia, D1.P34, s35

Stamouli, Maria, D1.P44, s39

Standage, Martyn, D1.S3.1(6), s5

Starkey, Michael, D2.P59, s111

Steell, Lewis, D1.P14, s25

Stensel, David J., D1.S3.1(4), D1.P18, D2.S4.2(5), s4, s27, s52

Stephens, Francis B., D2.S1.1(4), s64

Stephenson, Ben, D1.S3.2(4), D2.P60, s8, s111

Stevens, Rebecca C., D1.S3.4(5), s14

Stodden, David, D1.S3.1(2), s3

Stöggl, Thomas, D1.P49, s42

Stow, Mike, D2.S3.2, s1

Suckling, Craig, D2.P21, s93

Suga, Tadashi, D2.P27, s95

Sunderland, Caroline, D1.S2.2, D2.P24, s1, s94

Surmon, Sean, D2.S4.3(1), D2.P52, s54, s108

Swann, Christian, D1.S3.3(5), D2.P48, s12, s106

Swarén, Mikael, D1.P49, s42

Swinbourne, Richard, D2.P23, s93

Taehtinen, Richard, D2.S1.3(7), s73

Tajima, Fumihiro, D1.S3.2(4), s8

Talbot, Christopher, D2.S4.2(3), s52

Tallis, Jason, D1.P10, D1.P26., D2.P5, D2.P9, D2.P25, s23, s31, s85, s86, s94

Tamminen, Katherine, D1.S3.3(5), s12

Tas, Mart, D1.P42, s39

Taylor, Alyx, D2.P26, s95

Taylor, Lee, D1.S3.5(1), s16

Taylor, Richard J., D2.S4.5(4), D2.S1.5(6), D2.P53, s61, s81, s108

Tew, Garry, D1.P6, s21

Thackray, Alice, D2.S4.2(5), s52

Thake, Douglas C., D2.P53, s108

Theodorakis, Yannis, D1.P44, s39

Theodorou, Apostolos S., D2.S4.1(5), D2.S1.1(3), s49, s64

Thompson, Janice, D2.S1.2(5), s68

Tolfrey, Keith, D2.P60, s111

Tolfrey, Vicky, D1.S3.2(4), s8

Torregrossa, Miquel, D1.P33, D1.P34, D2.S4.3(4), D2.P44, D2. P49, s35, s55, s104, s106

Tottori, Nobuaki, D2.P27, s95

Towlson, Chris, D1.S3.5(1), s16

Tremblay, Mark S., D1.S3.1(6), s5

Triska, Christoph, D2.P61, s111

Tschan, Harald, D2.P61, s111

Tudor-Locke, Catrine, D1.S3.1(6), s5
Twist, Craig, D1.S3.2(2), D2.S4.2(2), D2.S4.5(2), D2.S4.5(5), D2.S1.5(5), D2.P56, s7, s51, s60, s61, s80, s109

Urquhart, Jack, D2.P57, s110

Van Hoye, Aurélie, D2.P40, s102

Van Rens, Fleur, D2.S4.3(5), s55

Van Rossem, Nicky, D1.P47, s41

Vansteenkiste, Maarten, D1.S3.4(3), s13

Vella, Stewart A., D1.S3.3(4), D1.S3.3(5), D2.P48, s11, s12, s106

Victoria, Chirstian, D1.P58, D2.S1.1(2), s46, s63

Viladrich, Carme, D2.P44, s104

Vine, Samuel, D1.P50, s42

Visscher, Chris, D1.P24, s31

Wadey, Ross, D2.S4.4(2), s57

Wagstaff, Christopher R. D., D2.S4.4(2), s57

Wainwright, Barney, D2.S1.1(3), s64

Waite, Samantha, D2.P6, s85

Wakefield, Caroline, D2.P36, s100

Walker, Leanne Jaye, D1.S3.1(2), D2.P15, s3, s89

Wallace-Sims, Tandiwe, D1.P52, s43

Wallis, Gareth A., D1.P5, s21

Walsh, Neil, D1.S2.2, s1

Watkins, Philip, D2.P16, s90

Watson, Matthew, D2.P4, s84

Weinstein, Meryle G., D1.P58, D2.S1.1(2), s46, s63

Welsh, James, D2.P62, s112

West, Sacha-Jane, D2.S1.1(1), s63

Westgate, Mike, D2.S1.3(5), s72

Weston, Miranda, D2.P18, s90

White, Rachel, D1.P54, s44

White, Ross, D2.P37, s101

Whiting, Stewart, D1.P21, s29

Wilcox, Chris, D1.S3.5(1), s16

Wiles, Jonathand., D2.P7, s86

Williams, Thomas, D2.S4.2(2), s51

Wilson, Laura, D1.P17, D2.S1.1(7), D2.P20, s27, s66, s92

Wilson, Mark, D1.P50, s42

Wilson, Nigel, D2.S1.4(5), s77

Winter, Stacy, D2.P39, s102

Wood, Alyssa, D2.P20, s92

Wood, Alyssia, D1.P17, s27

Wood, Daniel, D2.S1.5(7), s81

Wood, Nathan, D2.P50, s107

Woodman, Tim, D1.S3.3(6), s12

Wylleman, Paul, D1.P24, D1.P33, D1.P34, D1.P42, D1.P47, s31, s35, s39, s41

Yamatsu, Koji, D2.P51, s107

Yanaoka, Takuma, D1.S3.1(4), s4

Yavuz, Harley, D2.P25, s94

Young, Tom, D2.S1.3(6), s73

Yustres Amores, Inmaculada, D1.P55, D2.P63, s45, s112

Zakrzewski-Fruer, Julia, D1.S3.1(1), D1.S3.1(6), s3, s5

Zerdila, Diana, D2.S1.3(3), s72

Zhang, Shuge, D1.S3.3(6), s12

Zhao, Pei, D1.S3.1(6), s5

Zinchenko, Anastasia, D2.P21, s93

Zuch, Nicholas, D2.P64, s113 




\section{Alert me}

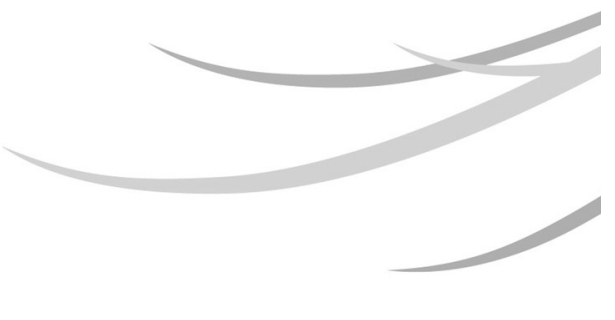

Taylor \& Francis Online offers a range of email and RSS alerts to keep you up to date with our publications

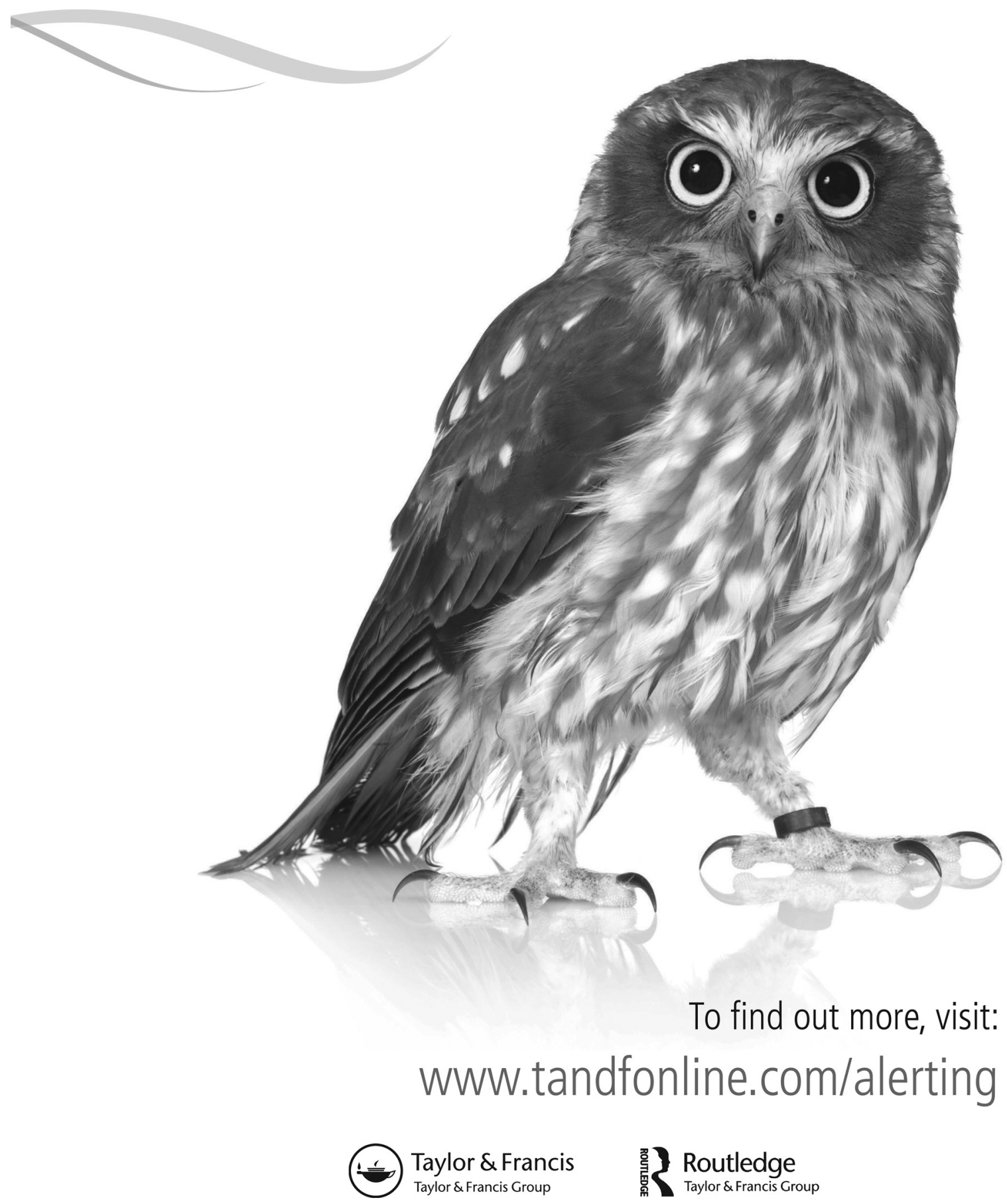


She knows about making

an impact 


\section{Make sure you are part of BASES Conference 2018}

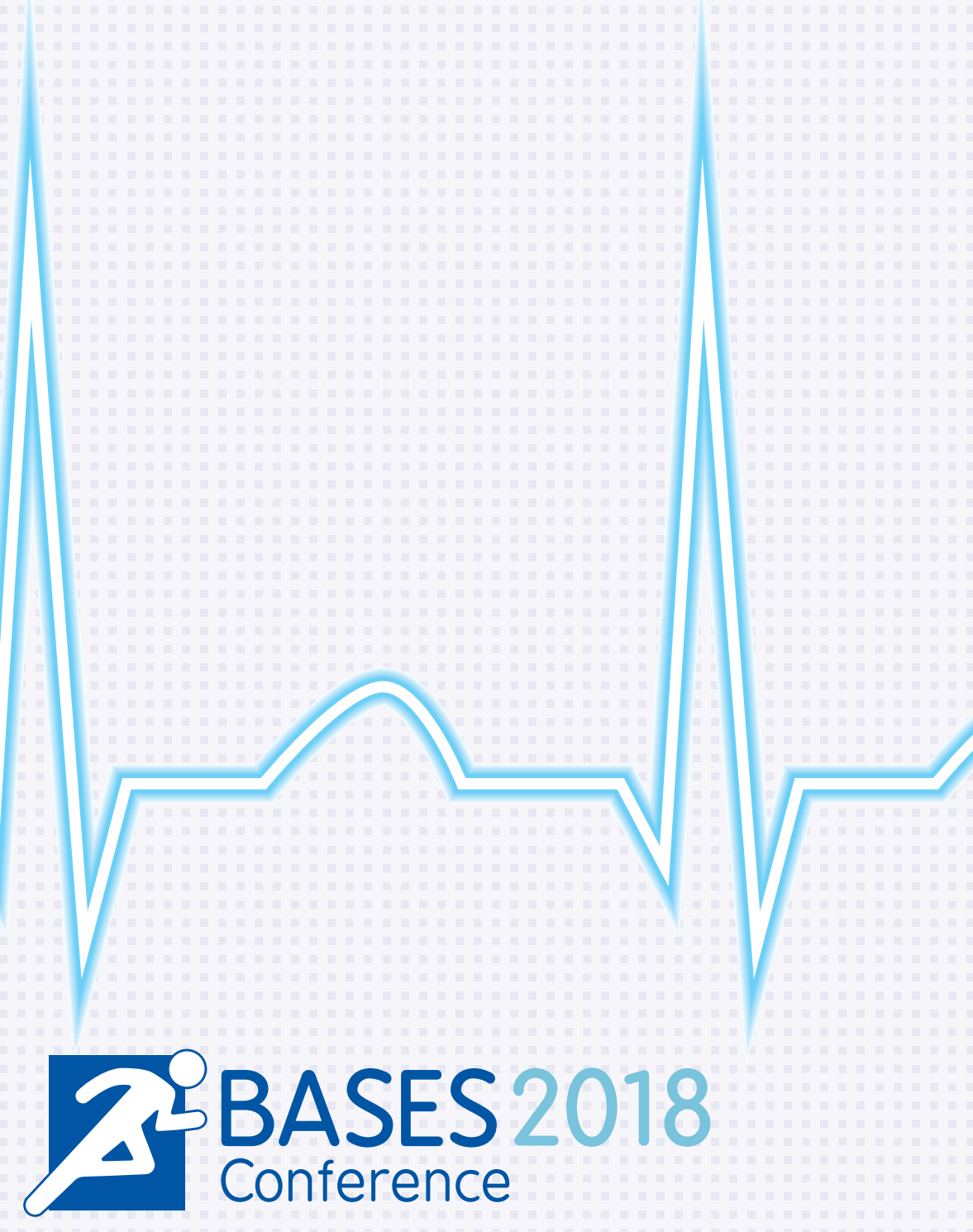

Tuesday 27 - Wednesday 28 November 2018

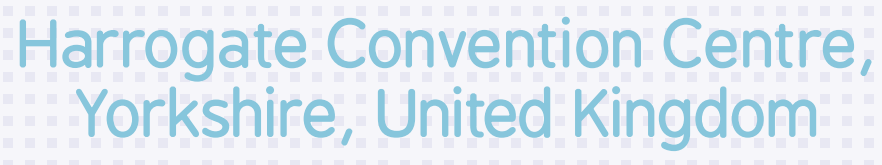

For further information

www.basesconference.co.uk

y @basesuk f $f$ /BASESUK (0) bases_uk in BASESUK 